Estabilidade assintótica para alguns modelos dissipativos de equações de placas

\author{
Marcio Antonio Jorge da Silva
}





\title{
Estabilidade assintótica para alguns modelos dissipativos de equações de placas $^{1}$
}

\author{
Marcio Antonio Jorge da Silva
}

\author{
Orientador: Prof. Dr. Ma To Fu
}

Tese apresentada ao Instituto de Ciências Matemáticas e de Computação - ICMC-USP, como parte dos requisitos para obtenção do título de Doutor em Ciências - Ciências de Computação e Matemática Computacional. VERSÃO REVISADA.

\section{USP - São Carlos}

Abril de 2012

\footnotetext{
${ }^{1}$ Este trabalho teve apoio financeiro da FAPESP sob o processo 2008/00123-7 de 01/06/2008 à 31/05/2010
} 
Ficha catalográfica elaborada pela Biblioteca Prof. Achille Bassi e Seção Técnica de Informática, ICMC/USP, com os dados fornecidos pelo(a) autor(a)

Jorge da Silva, Marcio Antonio
Estabilidade assintótica para alguns modelos
dissipativos de equaçós de placas / Marcio Antonio
Jorge da Silva; orientador Ma To Fu. -- São Carlos,
2012.
i a xii, 1 a $138 \mathrm{p}$.
Tese (Doutorado - Programa de Pós-Graduação em
Matemática) -- Instituto de Ciências Matemáticas e
de Computação, Universidade de São Paulo, 2012.
1. Equações Diferenciais Parciais. 2. Equações de
Placas. 3. Estabilidade Assintótica. 4. Pseudo
Laplaciano. 5. Memória. I. To Fu, Ma, orient. II.
Título.




\section{Agradecimentos}

Primeiramente agradeço a Deus pela oportunidade de concluir este trabalho em mais uma etapa de minha vida.

À toda minha família, em especial, à minha mãe que me deu condições para iniciar meus estudos acadêmicos sem que tivesse outros compromissos.

À minha esposa Naiara, pelo companheirismo, compreensão e cumplicidade. Também, por sempre me apoiar e entender os momentos de minha ausência perante aos estudos.

À todos os meus amigos de ex-república de Maringá e de São Carlos. Também, aos companheiros de estudos universitários de todos os tempos, em especial, aos colegas com tive o prazer de crescer pessoalmente e profissionalmente desde o início do doutorado em 2008.

À todos os professores do departamento de Matemática da UEM e do ICMC-USP que contribuiram na minha formação acadêmica desde a graduação até o doutorado.

Ao professor Dr. Doherty Andrade por me receber na UEM em 2009 em prol de minha ascensão profissional científica e pessoal. Ao professor Dr. Jaime E. Muñoz Rivera por me receber no LNCC em 2010 e coorientar em uma parte deste trabalho. Também, à professora Dra. Luci H. Fatori da UEL perante seus essenciais comentários com repeito a uma parte deste trabalho.

Aos professores Drs. Vittorino Pata, Roger Temam e Igor Chueshov, que mesmo não me conhecendo pessoalmente, enviaram-me alguns de seus trabalhos na área de pesquisa, o que contribuiu positivamente no desenvolvimento desta tese.

Ao meu orientador, e hoje um grande amigo, prof. Dr. Ma To Fu, pela orientação, paciência e pelos incontáveis conselhos. Também agradeço por sempre apoiar e querer o melhor para minha carreira acadêmica.

Por fim, agradeço a CAPES e, em especial, à FAPESP pelo apoio financeiro para realização deste trabalho. 

Neste trabalho estudamos questões relativas a existência, unicidade, dependência contínua, continuidade, taxas de decaimento e comportamento assintótico de soluções para uma classe de equações de placas lineares e não lineares. No primeiro capítulo revisamos alguns conteúdos e colecionamos uma série de resultados provenientes da teoria geral de análise funcional, semigrupos lineares e atratores, os quais serão aplicados ao longo desta tese. Nos dois próximos capítulos abordamos uma equação da placa de quarta ordem dissipativa com perturbações não lineares do tipo $p$ Laplaciano e localmente Lipschitz e com memória. No segundo capítulo provamos a estabilidade exponencial de energia correspondente ao problema homogêneo com memória de segunda ordem. Em seguida, no terceiro capítulo estabelecemos resultados que comprovam a existência de um atrator global com dimensão fractal finita para o sistema dinâmico associado ao problema com história de deslocamento relativo que equivale ao problema original. Finalmente, no quarto capítulo tratamos um modelo viscoelástico de placas de Mindlin-Timoshenko de segunda ordem. Nesta ocasião, consideramos essecialmente dois casos, o primeiro quando o sistema é totalmente dissipativo e, em seguida, quando o sistema é parcialmente dissipativo. No primeiro caso, determinamos que o semigrupo linear associado ao problema é analítico e, como consequência, é exponencialmente estável. No segundo caso, mostramos que o semigrupo perde decaimento exponencial e analiticidade, no entanto, provamos que as soluções possuem decaimento do tipo polinomial.

Palavras-chave: Equação da placa, modelo de Mindlin-Timoskenko, $p$ Laplaciano, memória, estabilidade assintótica, decaimento de energia, analiticidade, exponencialmente estável, estabilidade polinomial, conjunto absorvente, sistema dinâmico dissipativo, atrator global. 

In this work we study some questions concerning with existence, uniqueness, continuous dependence, continuity, rates of decay and asymptotic behavior of solutions for a class of linear and nonlinear plate equations. In the first chapter we review some concepts and collect a series of results provided from general theory of functional analysis, linear semigroups and attractors which will be applied throughout this thesis. In the next two chapters we discuss a damped plate equation of fourth order with nonlinear perturbations of the lower order of $p$-Laplacian type and locally Lipschitz, and a memory term. In the second chapter we prove the exponential stability of energy corresponding to the homogeneous problem with memory of second order. Then in the third chapter we establish some results that allow us to prove the existence of a global attractor with finite fractal dimension for dynamical system associated to the problem with relative displacement history which is equivalent to the original problem. Finally, in the fourth chapter we deal with a viscoelastic Mindlin-Timoshenko plate model of second order. At this moment we consider essentially two cases. The first one when the system is fully damped, then when the system is partially damped. In the first case we show that the semigroup associated to the Mindlin-Timoskenko system is analytic, which in particular implies exponential decay. In the second case we show that such semigroup loses exponential decay, also loses analyticity. However, we prove in this last case that the solutions have decay of polynomial type.

Keywords: Plate equation, Mindlin-Timoskenko model, $p$-Laplacian, memory, asymptotic behavior, energy decay, analyticity, exponentially stable, polynomial stability, absorbing set, dissipative dynamical systems, global attractor. 



\section{Geral}

$|\Omega|:=$ medida de Lebesgue de $\Omega \subset \mathbb{R}^{N} ;$

$|\alpha|=\alpha_{1}+\alpha_{2}+\cdots+\alpha_{N} \quad$ e $\quad \alpha !=\alpha_{1} ! \alpha_{2} ! \cdots \alpha_{N} !$ para todo $\alpha=\left(\alpha_{1}, \ldots, \alpha_{N}\right) \in \mathbb{N}^{N}$

$p^{\prime}=\frac{p}{p-1}$ expoente conjugado de $p ;$

$x^{\alpha}=x_{1}^{\alpha_{1}} x_{2}^{\alpha_{2}} \cdots x_{N}^{\alpha_{N}}$ para todo $x=\left(x_{1}, \ldots, x_{N}\right) \in \mathbb{R}^{N} ;$

$\operatorname{supp}(u)=\overline{\{x \in \Omega ; u(x) \neq 0\}^{\Omega}}$;

$\hookrightarrow$ inclusão contínua;

$\hookrightarrow \hookrightarrow$ inclusão compacta;

$\langle\cdot, \cdot\rangle$ dualidade;

$\left(X,\|\cdot\|_{X}\right)$ espaço de Banach.

\section{Operadores de derivação}

$$
\begin{aligned}
& D^{\alpha} u=\left\{\begin{array}{ccc}
\frac{\partial^{|\alpha|} u}{\partial x_{1}^{\alpha_{1}} \partial x_{2}^{\alpha_{2}} \ldots \partial x_{N}^{\alpha_{N}}} & \text { se } & \alpha \neq(0, \ldots, 0) ; \\
u & \text { se } & \alpha=(0, \ldots, 0) ;
\end{array}\right. \\
& \Delta u=\sum_{j=1}^{N} \frac{\partial^{2} u}{\partial x_{j}^{2}} \\
& \Delta^{2} u=\Delta(\Delta u) \\
& \Delta_{p} u=\operatorname{div}\left(|\nabla u|^{p-2} \nabla u\right) .
\end{aligned}
$$




\section{Espaços de funções}

$C(\Omega)=\{u: \Omega \rightarrow \mathbb{R} \mid u$ é contínua $\} ;$

$C^{k}(\Omega)=\{u: \Omega \rightarrow \mathbb{R} \mid u$ é $k$-vezes continuamente diferenciável $\} ;$

$C^{\infty}(\Omega)=\{u: \Omega \rightarrow \mathbb{R} \mid u$ é infinitamente diferenciável $\} ;$

$C_{0}^{k}(\Omega)=\left\{u \in C^{k}(\Omega) \mid \operatorname{supp}(u) \subset \Omega\right.$ é compacto $\}, \quad k \in \mathbb{N}$ ou $k=\infty ;$

$C^{k, \alpha}(\Omega)=\left\{u \in C^{k}(\Omega) \mid D^{k} u\right.$ é $\alpha$-Hölder contínua $\} ;$

$\mathcal{D}(\Omega):=$ espaço das funções teste;

$L^{p}(\Omega)=\left\{u: \Omega \rightarrow \mathbb{R} \mid u\right.$ é mensurável e $\left.\int_{\Omega}|u(x)|^{p} d x<\infty\right\} ;$

$L^{\infty}(\Omega)=\{u: \Omega \rightarrow \mathbb{R} \mid u$ é mensurável e $|u(x)| \leq K$ q.s. em $\Omega\} ;$

$W^{m, p}(\Omega)=\left\{u \in L^{p}(\Omega)\left|D^{\alpha} u \in L^{p}(\Omega), \quad 0 \leq\right| \alpha \mid \leq m\right\} ;$

$W_{0}^{m, p}(\Omega)={\overline{C_{0}^{\infty}(\Omega)}}^{W^{m, p}(\Omega)}$;

$H^{m}(\Omega)=W^{m, 2}(\Omega) ;$

$H_{0}^{m}(\Omega)=W_{0}^{m, 2}(\Omega) ;$

$L_{\mu}^{2}\left(\mathbb{R}^{+} ; X\right)=\left\{\eta: \mathbb{R}^{+} \rightarrow X \mid \int_{0}^{\infty} \mu(s)\|\eta(s)\|_{X}^{2} d s<\infty\right\} ;$

$L^{p}(0, T ; X)=\left\{u:(0, T) \rightarrow X \mid u\right.$ é mensurável e $\left.\int_{0}^{T}\|u(t)\|_{X}^{p} d t<\infty\right\} ;$

$L^{\infty}(0, T ; X)=\left\{u:(0, T) \rightarrow X \mid u\right.$ é mensurável e $\|u(t)\|_{X} \leq K$ q.s. em $\left.(0, T)\right\} ;$

$C([0, T], X)=\{u:[0, T] \rightarrow X \mid u$ é contínua de $[0, T]$ em $X\} ;$

$C^{k}([0, T], X)=\{u:[0, T] \rightarrow X \mid u$ é $k$-vezes continuamente diferenciável de $[0, T]$ em $X\} ;$

$C_{w}([0, T], X)=\{u:[0, T] \rightarrow X \mid u$ é fracamente contínua de $[0, T]$ em $X\} ;$

$\mathcal{L}(X, Y)=\{T: X \rightarrow Y \mid T$ é linear e contínua $\}$.

\section{Espaços duais}

$X^{\prime}=\mathcal{L}(X, \mathbb{R})$ dual de $X$

$\left[L^{p}(\Omega)\right]^{\prime}=L^{p^{\prime}}(\Omega), \quad 1 \leq p<\infty ;$

$\left[W_{0}^{m, p}(\Omega)\right]^{\prime}=W^{-m, p^{\prime}}(\Omega), \quad 1 \leq p<\infty, m \in \mathbb{N} ;$

$\left[H_{0}^{m}(\Omega)\right]^{\prime}=H^{-m}(\Omega), \quad m \in \mathbb{N} ;$

$\left[L^{p}(0, T, X)\right]^{\prime}=L^{p^{\prime}}\left(0, T, X^{\prime}\right), \quad 1 \leq p<\infty ;$ 
$\mathcal{D}^{\prime}(\Omega)=\mathcal{L}(\mathcal{D}(\Omega), \mathbb{R}) ;$

$\mathcal{D}^{\prime}(0, T ; X)=\mathcal{L}(\mathcal{D}(0, T), X)$.

\section{Convergências}

$\rightarrow$ convergência forte;

$\rightarrow$ convergência fraca;

$\stackrel{*}{\rightarrow}$ convergência fraca estrela.

\section{Memória}

$$
\begin{aligned}
& (g * u)(t)=\int_{0}^{t} g(t-s) u(x, s) d s ; \\
& (g \square u)(t)=\int_{0}^{t} g(t-s) \int_{\Omega}|u(x, t)-u(x, s)|^{2} d x d s .
\end{aligned}
$$

\section{Normas}

$$
\begin{array}{ll}
\|u\|_{p}=\left(\int_{\Omega}|u(x)|^{p} d x\right)^{1 / p}, & \|u\|_{\infty}=\sup _{x \in \Omega} \operatorname{ess}|u(x)| ; \\
\|u\|_{m, p}=\left(\sum_{|\alpha| \leq m}\left\|D^{\alpha} u\right\|_{p}^{p}\right)^{1 / p}, & \|u\|_{m, \infty}=\max _{|\alpha| \leq m}\left\|D^{\alpha} u\right\|_{\infty} ; \\
\|u\|_{W_{0}^{1, p}}=\|\nabla u\|_{p} ; & \quad\|u\|_{L^{\infty}(0, T ; X)}=\sup _{t \in(0, T)}\|u(t)\|_{X} ; \\
\|\eta\|_{\mu, X}=\left(\int_{0}^{\infty} \mu(s)\|\eta(s)\|_{X}^{2} d s\right)^{1 / 2} ; & \\
\|u\|_{L^{p}(0, T ; X)}=\left(\int_{0}^{T}\|u(t)\|_{X}^{p} d t\right)^{1 / p}, & \\
\|u\|_{C^{k}([0, T], X)}=\sum_{j=0}^{k} \max _{t \in[0, T]}\left\|\frac{d^{j} u(t)}{d t^{j}}\right\| \|_{X} ; & \sup _{x \in X,\|x\|_{X} \leq 1}|\langle\varphi, x\rangle| ; \\
\|\varphi\|_{X^{\prime}}=\sup _{x \in X,\|x\|_{X}=1}\|T x\|_{Y}=\sup _{x \in X, x \neq 0} \frac{\|T x\|_{Y}}{\|x\|_{X}} .
\end{array}
$$





\section{Sumário}

Resumo $\quad$ iii

$\begin{array}{lll}\text { Abstract } & \text { v }\end{array}$

Índice de Notações vii

$\begin{array}{ll}\text { Introdução } & 1\end{array}$

1 Preliminares 9

1.1 Espaços de Banach e de Hilbert . . . . . . . . . . . . . . . . . . . . . . . . 9

1.1.1 Um breve repasso ao conceito de topologia fraca e fraca estrela . . . . . . 14

1.2 Alguns resultados importantes . . . . . . . . . . . . . . . . . . . 15

1.3 O operador $\mathrm{p}$-Laplaciano . . . . . . . . . . . . . . . . . . . . . . . . . . . . 19

1.3.1 Uma desigualdade interessante . . . . . . . . . . . . . . . . . . . . . . . . . . . . . . . . . . .

1.4 Operadores lineares não limitados . . . . . . . . . . . . . . . . . . . . . 21

1.4.1 Operador $A$ associado à uma forma bilinear . . . . . . . . . . . . . . 22

1.4.2 Potências fracionárias do operador $A \ldots \ldots . \ldots 23$

1.5 Uma breve revisão sobre semigrupos lineares . . . . . . . . . . . . . . . . . 23

1.5.1 $C_{0}$-semigrupos de operadores lineares . . . . . . . . . . . . . . . . 24

1.5.2 Caracterização dos geradores infinitesimais de $C_{0}$-semigrupos $\ldots \ldots . .25$

1.5.3 Analiticidade e estabilidade assintótica de $C_{0}$-semigrupos $\ldots \ldots . \ldots 26$

1.5.4 Problema de Cauchy abstrato . . . . . . . . . . . . . . . . . 27

1.6 Uma breve revisão sobre atratores globais . . . . . . . . . . . . . . . 28

1.6.1 Condições suficientes para existência de atratores globais . . . . . . . . . . . 30

1.6.2 Dimensão fractal . . . . . . . . . . . . . . . . . . . . . . . 31 
2 Um modelo de placas com $p$-Laplaciano e memória 33

2.1 Introdução . . . . . . . . . . . . . . . . . . . . . 33

2.2 Hipóteses e notações iniciais . . . . . . . . . . . . . . . . . . . 34

2.2.1 Uma identidade para a memória . . . . . . . . . . . . . . . 35

2.3 Existência e unicidade . . . . . . . . . . . . . . . . . . 36

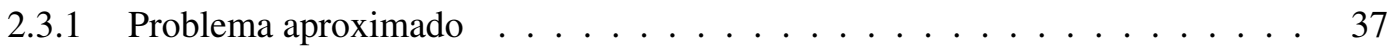

2.3 .2 Estimativa a priori $1 \ldots \ldots \ldots \ldots$

2.3.3 Passagem ao limite e solução fraca . . . . . . . . . . . . . . . 38

2.3 .4 Estimativa a priori $2 \ldots \ldots \ldots \ldots \ldots$

2.3.5 Passagem ao limite e solução fraca mais regular . . . . . . . . . . . . 46

2.3 .6 Unicidade . . . . . . . . . . . . . . . . . . . . . . . . . . 46

2.4 Decaimento exponencial de Energia $\ldots \ldots \ldots \ldots \ldots$

3 Um modelo de placas com $p$-Laplaciano e memória com história 53

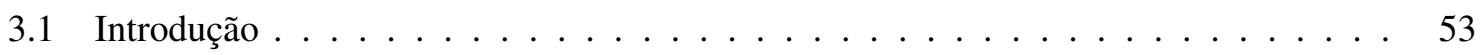

3.2 Hipóteses e notações iniciais . . . . . . . . . . . . . . . . . 55

3.3 Existência, unicidade e dependência contínua . . . . . . . . . . . . 56

$3.3 .1 \quad$ Problema aproximado . . . . . . . . . . . . . . . 57

3.3 .2 Estimativa a priori $1 \ldots \ldots \ldots \ldots \ldots$

3.3.3 Passagem ao limite e solução fraca . . . . . . . . . . . . . . . . 67

3.3 .4 Estimativa a priori $2 \ldots \ldots \ldots \ldots \ldots$

3.3.5 Passagem ao limite e solução fraca mais regular . . . . . . . . . . . . 80

3.3.6 Dependência contínua e unicidade . . . . . . . . . . . . . . . . . . . . . 84

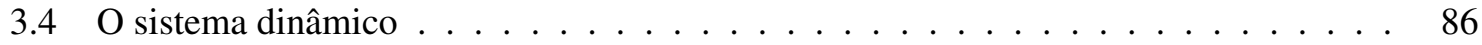

3.4.1 Existência de conjunto absorvente . . . . . . . . . . . . . . . . . . 87

3.5 Um resultado de estabilização . . . . . . . . . . . . . . . . . . . . . . . 92

3.6 Atrator Global . . . . . . . . . . . . . . . . . . . . . . . . . . . . . 96

3.6.1 Dimensão fractal finita . . . . . . . . . . . . . . . . . . . . 99

4 Um modelo de placas de Mindlin-Timoshenko 105

4.1 Introdução . . . . . . . . . . . . . . . . . . . . . . . . . 105

4.2 Existência e unicidade . . . . . . . . . . . . . . . . . . 107

4.3 Analiticidade . . . . . . . . . . . . . . . . . . . . . . . . . 112

4.3.1 Estabilidade exponencial . . . . . . . . . . . . . . . . . 114

4.4 Falta de estabilidade exponencial . . . . . . . . . . . . . . . . . . . . 115

4.5 Decaimento polinomial . . . . . . . . . . . . . . . . . . . . . 118

4.5 .1 Otimalidade . . . . . . . . . . . . . . . . . . . . . . 127

Referências Bibliográficas $\quad 129$

$\begin{array}{ll}\text { Índice Remissivo } & 137\end{array}$ 


\section{Introdução}

Nos últimos anos muitos estudos sobre a equação da onda (de segunda ordem) e equação da placa (de quarta ordem), e também perturbações não lineares destas, tem sido desenvolvidos por pesquisadores dentro do contexto de equações de evolução (de segunda ordem com respeito ao tempo $t>0$ ). Analisar questões relacionadas a existência, unicidade e dependência contínua de Problemas de Valor Inicial e de Fronteira (PVIF) constitui um primeiro passo na abordagem dos problemas dentro linha de pesquisa de equações diferenciais parciais. Num segundo momento, faz-se necessário estudar também as propriedades (qualitativas) das soluções globais obtidas a priori, como por exemplo, o comportamento assintótico de soluções que consiste, basicamente, em decaimento exponencial ou polinomial de soluções, ou ainda, a existência de atratores globais (e suas "medidas" finito-dimensionais) para o sistema dinâmico gerado por tais soluções do PVIF, além de outras abordagens. Por outro lado, quando a solução de um problema é local, então alguns autores estudam também a taxa com que essa solução explode em tempo finito. Este último caso não será tratado neste trabalho.

Para ser um pouco mais específico, equações de placas com perturbação não linear do tipo $\phi$ Laplaciano

$$
u_{t t}+\Delta_{x}^{2} u-\operatorname{div}_{x}\left(\phi\left(\nabla_{x} u\right)\right)=\mathcal{F}\left(x, u, u_{t}\right)
$$

onde $\phi(z) \approx|z|^{(p-2)} z, p \geq 2$, e $\mathcal{F}\left(x, u, u_{t}\right)$ representa, adicionalmente, uma força externa e/ou uma dissipação linear(es) ou não linear(es), têm atraido atenção de vários autores com respeito a existência e comportamento assintótico de soluções. Neste caso, podemos dizer que o operador $\operatorname{div}_{x}\left(\phi\left(\nabla_{x} u\right)\right)$ surge como uma perturbação não linear de menor ordem do operador biharmônico $\Delta_{x}^{2}=\Delta_{x}\left(\Delta_{x}\right)$ na equação (1).

No caso de equações da onda regida pelo operador $\phi$-Laplaciano com dissipação viscosa $-\Delta_{x} u_{t}$, há alguns trabalhos pioneiros como os de Greenberg et al [53, 54] em dimensão 1. Em dimensões maiores, podemos citar os trabalhos de Tsutsumi [92] e Clements [28]. Depois disso, surgiram vários outros autores que abordaram problemas correlatos, como por exemplo, nos trabalhos $[7,12,13,32,34,44,72,73,77,84,87]$. 
Retornando à equação (1), vemos que esta é um protótipo de diversos modelos importantes que se aplicam no mundo real no que diz respeito a perturbações da equação da placa. Para exemplificar isto, vejamos os seguintes casos:

No caso bidimensional, a seguinte equação da placa não linear referente um modelo de KirchhoffBoussinesq

$$
u_{t t}+k u_{t}+\Delta^{2} u=\operatorname{div}\left\{|\nabla u|^{2} \nabla u\right\}+\sigma \Delta\left\{f_{1}(u)\right\}-f_{2}(u),
$$

definida sobre um domínio limitado $\mathbb{R}^{2}$, considerando três condições de fronteira, a saber, livre, fixada e apoiada (free, clamped ou simply supported boundary conditions), foi abordada por Chueshov \& Lasiecka em [23, 27]. Nesta ocasião, os autores apresentaram um estudo sobre a boa colocação do problema segundo Hadamard e o comportamento assintótico de soluções fracas e fortes (existência de atrator global), considerando apenas uma dissipação fraca (weak damping) do tipo friccional $k u_{t}$. Convém observar ainda, como notado pelos autores em [23, 27] e justificado com mais detalhes em [25, Capítulo 7], que o modelo acima surge naturalmente como um caso limite das equações de Mindlin-Timoshenko que descrevem a dinâmica de uma placa submetida a efeitos de cisalhamento transversal, ver Lagnese et al $[64,65]$ para maiores detalhes. Com respeito ao modelo de placas de Mindlin-Timoshenko faremos uma abordagem diferenciada mais adiante.

No caso $N$-dimensional, Yang et al $[96,97]$ trataram uma classe de modelos de Kirchhoff por meio da equação

$$
u_{t t}+\Delta^{2} u-\operatorname{div}\left\{|\nabla u|^{m-1} \nabla u\right\}-\Delta u_{t}=h\left(x, u, u_{t}\right),
$$

definida sobre um domínio limitado de $\mathbb{R}^{N}$, com $N \geq 1$ natural e $m \geq 1$ delimitado por uma constante que depende de $N$ em dimensões maiores, considerando condições de fronteira fixadas ou apoiadas (clamped ou simply supported boundary conditions). Os principais resultados apresentados nesta ocasião consistem em determinar existência global e o comportamento assintótico de soluções fracas e mais regulares (existência de atrator global), considerando uma dissipação forte (strong damping) do tipo viscosa $\Delta u_{t}$. Mais recentemente, Yang [98, 99] estudou questões relativas a dimensionalidade de tais atratores, como por exemplo, as dimensões de Hausdorff e fractal.

Para completar o raciocínio, lembramos que um modelo correspondente ao fluxo de microestruturas elastoplásticas da forma

$$
u_{t t}+u_{x x x x}+a\left(\left|u_{x}\right|^{2}\right)_{x}=0,
$$

foi introduzido por An \& Peirce [2, 3] no caso unidimensional, onde $a>0$. Assim sendo, ao enxergar o termo $a\left(\left|u_{x}\right|^{2}\right)_{x}$ como um operador do tipo $p$-Laplaciano, notamos que este último modelo difere dos dois anteriores pelo sinal da perturbação proveniente do operador $p$-Laplaciano.

Pelos trabalhos acima mencionados podemos imaginar que em dimensões maiores $(N \geq 3)$ a dissipação forte, $-\Delta u_{t}$, constitui um importante papel no modelo de placa com perturbação de menor ordem do tipo $p$-Laplaciano

$$
u_{t t}+\alpha \Delta^{2} u-\Delta_{p} u=\mathcal{F}\left(x, u, u_{t}\right),
$$

onde

$$
\Delta_{p} u=\operatorname{div}\left(|\nabla u|^{p-2} \nabla u\right), \quad p \geq 2,
$$


e $\alpha>0$ é uma constante. De fato, para $\alpha=1$ a existência global e unicidade de soluções podem ser verificadas adicionando uma dissipação forte $-\Delta u_{t}$, assim como Yang et al [96, 97]. Contudo, se considerarmos apenas uma dissipação fraca $u_{t}$, então parece ser difícil de obter unicidade e a continuidade de soluções globais no caso $N$-dimensional, para $N \geq 3$. Ao contrário disto, para dimensões menores, $N=1$ ou $N=2$, a boa colocação do problema (2) implementando apenas uma dissipação fraca já é bem conhecido, como exposto por Chueshov \& Lasiecka [23] e Yang [95].

Motivados pelas obras citadas acima, o presente trabalho procura fornecer resultados relacionados a existência global de soluções para a equação (2) sob o efeito de um termo de memória. Em seguida, buscaremos as propriedades qualitativas das soluções com respeito a estabilidade assintótica ao longo do tempo. A relevância de nosso trabalho se deve ao fato de que, além de tomar um certo cuidado com as ferramentas ao se trabalhar com o problema viscoelástico no caso $N$-dimensional, devemos avaliar a interação do termo de memória com os operadores $p$-Laplaciano e biharmônico. Pensamos que estas considerações, além de outras que citaremos a seguir, não foram consideradas anteriormente por outros autores.

De um modo mais conciso, estudaremos num primeiro momento a existência de soluções e decaimento de energia para equação (2) em um domínio limitado de $\mathbb{R}^{N}$, considerando

$$
\mathcal{F}\left(x, u, u_{t}\right):=-(g * \Delta u)(t)+\Delta u_{t}-f(u),
$$

onde a convolução em (3), representando um termo de memória não local (que eventualmente chamaremos neste trabalho de memória sem história), é dada por

$$
(g * y)(t)=\int_{0}^{t} g(t-s) y(s) d s, \quad t>0,
$$

e a função $g$ é chamada de núcleo da memória. Assim sendo, combinando (2) e (3), estudamos no Capítulo 2 um problema de valor inicial e de fronteira para a seguinte equação da placa não linear com memória

$$
u_{t t}+\Delta^{2} u-\Delta_{p} u+\int_{0}^{t} g(t-s) \Delta u(s) d s-\Delta u_{t}+f(u)=0
$$

em $\Omega \times(0,+\infty)$, onde $\alpha=1$ e $\Omega \subset \mathbb{R}^{N}$ é um domínio limitado.

A existência de soluções para (4) é garantida pelo Teorema 2.3.1, usando o método de FaedoGalerkin, ver por exemplo Yang et al [96, 97], Cavalcanti et al [19], ou ainda, os clássicos Lions [66] e Lions \& Magenes [67]. O decaimento exponencial de energia é assegurado pelo Teorema 2.4.1, onde usamos o método de energia perturbada, ver por exemplo Cavalcanti et al $[18,19] \mathrm{e}$ Zuazua et al [56, 100]. É importante ressaltar que o procedimento utilizado para estudar (4) gerou, numa primeira circunstância, uma certa dificuldade técnica ao considerarmos um termo de memória do tipo $\int_{0}^{t} g(t-s) \Delta^{2} u(s) d s$. Contudo, com um pouco mais de investigação, também podemos concluir a veracidade dos resultados de existência e comportamento assintótico substituindo o termo de memória de segunda ordem $\int_{0}^{t} g(t-s) \Delta u(s) d s$ por um termo de memória de quarta ordem $\int_{0}^{t} g(t-s) \Delta^{2} u(s) d s$, a menos do sinal. Os resultados referentes ao Capítulo 2 (Teoremas 2.3.1 e 2.4.1) foram publicados na revista Mathematical Methods in the Applied Sciences, conforme a referência [5]. 
Existe hoje uma ampla literatura que aborda questões relativas ao conceito de estabilidade em viscoelasticidade para equações da onda e da placa. A título de exemplo, citamos os seguintes trabalhos $[6,20,30,31,71,78,80]$ e suas referências.

Prosseguindo, nosso objetivo num segundo momento foi de estender os conceitos estabelecidos (anteriormente) em [5], acoplando à equação (2) uma memória com história para $t<0$, de onde teremos de introduzir a história com deslocamento relativo. O motivo para tal pretenção é que a equação (4) não corresponde a um sistema autônomo, justamente pela convolução $(g * \Delta u)(t)=$ $\int_{0}^{t} g(t-s) \Delta u(s) d s$, que depende de $t>0$, e é de segunda ordem. Com isto, não poderiamos estudar a dinâmica assintótica para as soluções do problema por meio da teoria estabelecida para problemas autônomos. No entanto, para contornar essa situação, estudamos a existência de soluções para a equação (2) em um domínio limitado de $\mathbb{R}^{N}$, definindo $\mathcal{F}\left(x, u, u_{t}\right)$ como

$$
\mathcal{F}\left(x, u, u_{t}\right):=\int_{-\infty}^{t} \mu(t-s) \Delta^{2} u(s) d s+\Delta u_{t}-f(u)+h(x) .
$$

O comportamento assintótico de soluções também foi estudado quando a força externa $h$ é identicamente nula, ou seja, quando $h \equiv 0$. Note ainda que o termo integral (memória original) que surge em (5) pode ser reescrito formalmente como

$$
\int_{-\infty}^{t} \mu(t-s) \Delta^{2} u(s) d s=\int_{0}^{\infty} \mu(s) \Delta^{2} u(t-s) d s .
$$

Logo, combinando a equação (2) com as identidades (5)-(6), chegamos a seguinte classe de equações da placa não linear com memória (num contexto com história para $t<0$ )

$$
u_{t t}+\alpha \Delta^{2} u-\Delta_{p} u-\int_{0}^{\infty} \mu(s) \Delta^{2} u(t-s) d s-\Delta u_{t}+f(u)=h
$$

em $\Omega \times(0,+\infty)$, com condições iniciais e de fronteira estabelecidas no Capítulo 3, onde agora $\alpha=\alpha(0)>0$ é uma constante interligada ao núcleo da memória $\mu$ e $\Omega \subset \mathbb{R}^{N}$ é um domínio limitado.

Mais ainda, usando um argumento introduzido por Dafermos [30], podemos transformar o problema com história em um problema autônomo equivalente e, assim, estudar a existência de atratores globais para as soluções de tal problema equivalente. Outros autores também já se utilizaram deste argumento, como por exemplo nos trabalhos de Giorgi et al [47, 48, 49, 50, 51], ou ainda, no interessante e bem apresentado artigo de Grasselli \& Pata [52], onde os autores estabeleram de forma padrão o papel da equação suplementar e o retorno à equação original. Ver também Pata \& Zucchi [83]. De um modo mais preciso, definimos uma nova variável ao sistema por

$$
\eta=\eta^{t}(x, s)=u(x, t)-u(x, t-s), \quad x \in \Omega, s>0, \quad t \geq 0,
$$

também conhecida como história de deslocamento relativo. Então, depois de alguns ajustes como veremos no Capítulo 3, podemos converter a equação (7) no seguinte sistema equivalente

$$
\begin{aligned}
& u_{t t}+\Delta^{2} u-\Delta_{p} u+\int_{0}^{\infty} \mu(s) \Delta^{2} \eta^{t}(s) d s-\Delta u_{t}+f(u)=h, \\
& \eta_{t}=-\eta_{s}+u_{t}
\end{aligned}
$$


com condições iniciais e de fronteira correspondentes. Com isto, nosso próximo objetivo foi de apresentar resultados que complementam àqueles determinados em [5], estudando o problema (9)(10) sob aspéctos de existência e comportamento assintótico.

O Teorema 3.3.1 do Capítulo 3 garante que o problema (9)-(10) é bem posto, onde também usamos o método de Faedo-Galerkin. Quando $h \equiv 0$, a estabilidade assintótica também é assegurada usando o método de energia perturbada, conforme Proposição 3.4.1 e Observação 3.4.1. Vale ressaltar que neste caso usa-se somente as hipóteses atribuidas para o núcleo da memória $\mu$ (ver Capítulo 3 ) e não mais a identidade envolvendo a memória do problema (4) (ver Capítulo 2). Estes resultados (Teorema 3.3.1 e a Proposição 3.4.1 em conjunto com a Observação 3.4.1) foram aceitos para publicação na revista IMA Journal of Applied Mathematics, conforme a referência [58]. Além disso, quando $h \neq 0$ é independente da variável $t$, então as Proposições 3.4.1 e 3.5.1 garantem a existência de um conjunto absorvente e uma estabilização para as soluções do problema (9)-(10), respectivamente. Mais ainda, os Teoremas 3.6.1 e 3.6.2 determinam a existência de um atrator global com dimensão fractal finita para as soluções de (9)-(10), por meio de uma aplicação da teoria abstrata para atratores, com base nos trabalhos de Ceron \& Lopes [21], Chueshov \& Lasiecka [22, 25] e Hale [55]. Ver também os clássicos Babin \& Vishiki [8] e Temam [91]. Os últimos resultados acima citados, referente ao Capítulo 3, serão submetidos para publicação segundo a referência [59].

Neste momento, vale a pena notar que a nossa abordagem para a equação de placas perturbada pelo operador não linear $p$-Laplaciano e pela não linearidade localmente Lipschitz $f(u)$ e com um termo de memória (ver a equação (4) e o sistema (9)-(10)) é diferenciada das expostas em Giorgi et al [50], Pata \& Zucchi [83] e Yang [98, 99], devido ao fato de que em alguns deles é requerido regularidade de classe $C^{1}$ para o semigrupo de evolução associado ao problema, alguns impõem condições sobre a derivada $f^{\prime}$ da função $f$ e outros pelos resultados utilizados aos espaços de fase envolvidos no problema. Com isto, podemos dizer que nossos estudos amplia a classe de problemas abordados pelos autores acima, com respeito a existência e comportamento assintótico para um problema com memória. Para saltar as dificuldades encontradas, forneceremos um resultado de estabilização que segue ideias recentes devido aos trabalhos de Chueshov \& Lasiecka [22, 25] e Pata \& Zelik [82]. Ver também Hale [55]. Contudo, devido ao termo de memória tratada em nossos problemas, nosso trabalho difere destes últimos citados, contribuindo também em ampliar a classe de problemas que possuem aplicações da teoria geral de atratores.

Conforme podemos ver, existem hoje uma ampla gama de resultados abstratos que dizem respeito à existência de atratores globais. A título de exemplo, citamos os seguintes trabalhos $[8,21,22,25,35,55,74,82,91,94]$ e suas referências. Além disso, muitos desses resultados têm se mostrado eficientes quando aplicados dentro do contexto de equações diferenciais parciais.

Caminhando por outro lado, nos deparamos com uma forma de modelar a equação da placa no caso bidimensional $(N=2)$ por meio de um sistema de equações de segunda ordem (do tipo "equações de ondas"), a saber, o modelo de placas finas de Mindlin-Timoshenko como tratado (com dissipação na fronteira) em Lagnese [64]. Ver também Lagnese \& Lions [65]. Mais precisamente, 
consideremos o seguinte modelo de placas de Mindlin-Timoshenko

$$
\begin{aligned}
& \rho h w_{t t}-K\left[\frac{\partial}{\partial x}\left(\psi+\frac{\partial w}{\partial x}\right)+\frac{\partial}{\partial y}\left(\varphi+\frac{\partial w}{\partial y}\right)\right]=0 \\
& \frac{\rho h^{3}}{12} \psi_{t t}-D\left(\frac{\partial^{2} \psi}{\partial x^{2}}+\frac{1-\mu}{2} \frac{\partial^{2} \psi}{\partial y^{2}}+\frac{1+\mu}{2} \frac{\partial^{2} \varphi}{\partial x \partial y}\right)+K\left(\psi+\frac{\partial w}{\partial x}\right)=0 \\
& \frac{\rho h^{3}}{12} \varphi_{t t}-D\left(\frac{\partial^{2} \varphi}{\partial y^{2}}+\frac{1-\mu}{2} \frac{\partial^{2} \varphi}{\partial x^{2}}+\frac{1+\mu}{2} \frac{\partial^{2} \psi}{\partial x \partial y}\right)+K\left(\varphi+\frac{\partial w}{\partial y}\right)=0
\end{aligned}
$$

em $\Omega \times(0, \infty)$, onde $\Omega$ é um domínio limitado de $\mathbb{R}^{2}$. As constantes positivas $\rho$ simboliza a massa por unidade de volume, $h$ representa a espessura uniforme da placa, $K$ exprime o módulo de cisalhamento, $D$ denota o módulo de rigidez à flexão e a constante $0<\mu<1 / 2$ é chamada coeficiente de Poisson, sendo proveniente de considerações físicas sobre a placa, ver Lagnese et al [64, 65]. Já as funções $\psi=\psi(x, y, t)$ e $\varphi=\varphi(x, y, t)$ representam os ângulos de rotação de um filamento da placa e a função $w=w(x, y, t)$ denota o deslocamento transversal da superfície média da placa, para $(x, y) \in \Omega$ e $t \geq 0$.

Note que o sistema linear (11)-(13) é conservativo, então para obter resultados de estabilidade para soluções deste alguns autores introduziram dissipações na fronteira do conjunto $\Omega$. Por exemplo, em Lagnese [64] foi considerado uma condição de estabilização na fronteira por meio de um controle "feedback" e condições geométricas na fronteira. Isto permitiu o autor obter comportamento assintótico de energia. Neste mesmo sentido, Muñoz Rivera \& Oquendo [80] consideraram o sistema (11)-(13) com dissipações viscoelásticas na fronteira (memória). Com isto, os autores mostraram que a energia associada ao problema é estável com estabilidade assintótica sendo da mesma forma que a taxa de decaimento imposta ao núcleo da memória.

Mais recentemente, Fernández Sare [41] estudou o sistema (11)-(13) sob dissipações do tipo friccionais $d_{1} \psi_{t}$ e $d_{2} \varphi_{t}$ nas equações (12) e (13), e somente usando condições de fronteira de Dirichlet. Nesta ocasião, o autor somente deduziu a falta de estabilidade exponencial para soluções do sistema parcialmente dissipativo e, fazendo uso de técnicas multiplicativas juntamente com um resultado abstrato devido a Bátkai et al [11], obteve estabilidade polinomial de energia, onde a taxa de decaimento pode ser melhorada de acordo com a regularidade dos dados inciciais.

Tendo as referências acima citadas como motivação, apresentamos no Capítulo 4, sob a coorientação do Professor Dr. Jaime E. Muñoz Rivera, resultados que lidam com taxas de decaimento de soluções para uma versão dissipativa do sistema (11)-(13) de um modo independente aos trabalhos anteriores com respeito às taxas de decaimento. Tais resultados são baseados no fato de considerarmos um sistema totalmente e parcialmente dissipativo. A contribuição neste caso se deve ao fato de que abordar o conceito de analiticidade para o sistema (dissipativo) bem como estabelecer taxas de estabilidade, usando somente a teoria abstrata para semigrupos lineares, não foram consideradas anteriormente por outros autores no caso apresentado nesta tese. 
De um modo mais sucinto, denotando por $\mathcal{L}_{1}, \mathcal{L}_{2}$ e $\mathcal{L}_{3}$ os seguintes operadores lineares elípticos de segunda ordem

$$
\begin{aligned}
& \mathcal{L}_{1}(\psi, \varphi, w)=\frac{\partial}{\partial x}\left(\psi+\frac{\partial w}{\partial x}\right)+\frac{\partial}{\partial y}\left(\varphi+\frac{\partial w}{\partial y}\right), \\
& \mathcal{L}_{2}(\psi, \varphi)=\frac{\partial^{2} \psi}{\partial x^{2}}+\frac{1-\mu}{2} \frac{\partial^{2} \psi}{\partial y^{2}}+\frac{1+\mu}{2} \frac{\partial^{2} \varphi}{\partial x \partial y} \\
& \mathcal{L}_{3}(\varphi, \psi)=\frac{\partial^{2} \varphi}{\partial y^{2}}+\frac{1-\mu}{2} \frac{\partial^{2} \varphi}{\partial x^{2}}+\frac{1+\mu}{2} \frac{\partial^{2} \psi}{\partial x \partial y}
\end{aligned}
$$

estudamos o comportamento assintótico de soluções para o seguinte sistema de placas de MindlinTimoshenko com dissipação viscosa

$$
\begin{aligned}
& \rho h w_{t t}-K \mathcal{L}_{1}(\psi, \varphi, w)-D_{0} \Delta w_{t}=0 \\
& \frac{\rho h^{3}}{12} \psi_{t t}-D \mathcal{L}_{2}(\psi, \varphi)+K\left(\psi+\frac{\partial w}{\partial x}\right)-D_{1} \mathcal{L}_{2}\left(\psi_{t}, \varphi_{t}\right)=0 \\
& \frac{\rho h^{3}}{12} \varphi_{t t}-D \mathcal{L}_{3}(\varphi, \psi)+K\left(\varphi+\frac{\partial w}{\partial y}\right)-D_{1} \mathcal{L}_{3}\left(\varphi_{t}, \psi_{t}\right)=0
\end{aligned}
$$

em $\Omega \times(0, \infty)$, com condições iniciais e de fronteira estabelecidas no Capítulo 4 .

O Teorema 4.2.2 assegura a existência de soluções para (14)-(16) baseando-se no método de semigrupos lineares, de forma inteiramente análoga ao trabalho de Lagnese [64, Capítulo 3]. Ver também vários outros exemplos em Liu \& Zheng [70] e Muñoz Rivera [79]. Em seguida, usando resultados abstratos ([70, Capítulo 1]) mostraremos que o semigrupo associado ao sistema é analítico e possui estabilidade exponencial quando o sistema é totalmente dissipativo $\left(D_{0}, D_{1}>0\right)$, conforme o Teorema 4.3.1 e Corolário 4.3.2. Além disso, mostramos por meio do Teorema 4.4.1 que o semigrupo correspondente ao sistema parcialmente dissipativo $\left(D_{0}=0\right.$ e $\left.D_{1}>0\right)$ não é exponencialmente estável. Contudo, usando um resultado recente devido a Borichev \& Tomilov [14], determinamos neste último caso que as soluções possuem decaimento do tipo polinomial com taxa de decaimento podendo ser melhorada de acordo com a regularidade dos dados iniciais. No entanto, veremos ainda que se fixarmos os dados iniciais, então a taxa de decaimento polinomial não pode ser melhorada, o que nos dá otimalidade de decaimento polinomial (ver os Teoremas 4.5.6 e 4.5.7).

Vale pena notar que todos os resultados apresentados nesta parte são verdadeiros independentemente das relações entres as constantes do sistema, o que não é comum no caso unidimensional $(N=1)$ em problemas correlatos, ver por exemplo Muñoz Rivera \& Racke [81] para um modelo de Timoshenko e, também, Fatori \& Muñoz Rivera [40] para um sistema termoelástico de Bresse. Além disso, os resultados de estabilidade assintótica determinados no Capítulo 4 com respeito ao sistema (14)-(16) diferem dos, até então, estabelecidos para versões (dissipativas) do sistema de Mindlin-Timoshenko, como citado acima. Todos os resultados referente a este capítulo tiveram a supervisão do professor Dr. Jaime E. Muñoz Rivera e, em breve, serão enviados para publicação segundo a referência [60]. Essa última abordagem, que consiste em obter taxas de decaimento para um sistema como aplicação da teria espectral de geradores infinitesimais, vem sendo mais considerada por alguns autores nestes últimos anos. Como exemplo, podemos citar os trabalhos $[11,39,40,41,68,69,70,81,86]$ e suas referências. 



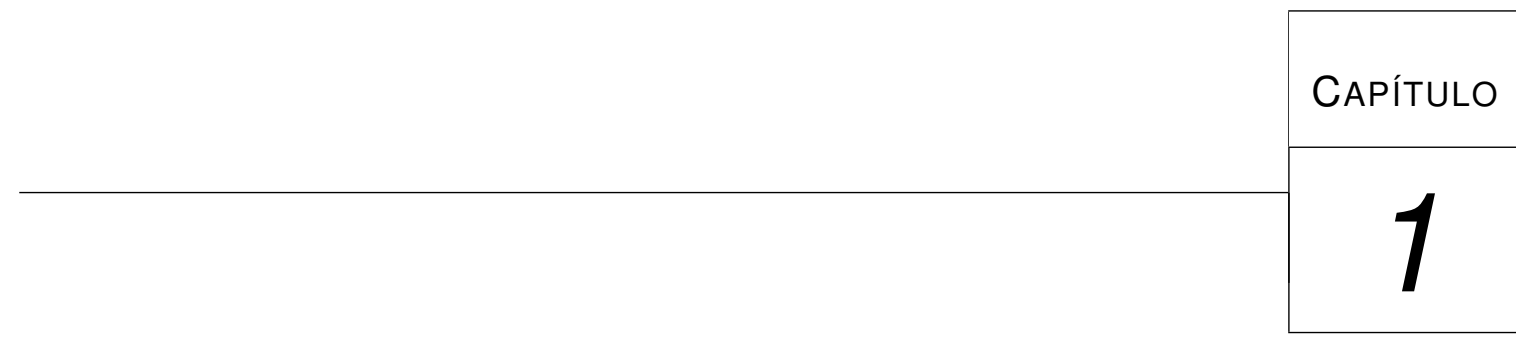

\section{Preliminares}

Este capítulo inicial tem um caráter introdutório e o leitor mais experiente pode omitir a leitura do mesmo, passando aos capítulos seguintes. Aqui vamos introduzir as notações que serão utilizadas em toda a tese bem como apresentar alguns dos resultados mais clássicos da teoria geral de análise não linear, análise funcional, espaços de Sobolev, semigrupos lineares, semigrupos não lineares e atratores. Faremos uso de tais resultados nos capítulos seguintes para facilitar a compreensão do conteúdo abordado e, também, com o objetivo de tornar este trabalho o mais autossuficiente possível. Os resultados (Teoremas, Proposições, Lemas, etc...) serão apresentados sem uma prova formal, porém forneceremos em cada seção as referências clássicas dentro da literatura para os conceitos citados. Também mencionaremos algumas referências em português que contribuiram para o desenvolvimento deste trabalho.

\subsection{Espaços de Banach e de Hilbert}

Iniciaremos com alguns conceitos básicos da teoria dos espaços de Sobolev e análise funcional, seguindo as referências Adams \& Fournier [1], Brézis [15], Folland [43], Lions [66] e Medeiros [75].

Seja $\Omega \subset \mathbb{R}^{N}$ um aberto. Para $1 \leq p<\infty$ denotamos por $L^{p}(\Omega)$ o conjunto (das classes) de funções Lebesgue mensuráveis $u$, tais que $|u|^{p}$ é uma função integrável sobre $\Omega$. Em termos de conjuntos,

$$
L^{p}(\Omega)=\left\{u: \Omega \rightarrow \mathbb{R} \mid u \text { é mensurável, } \int_{\Omega}|u(x)|^{p} d x<\infty\right\} .
$$


$\operatorname{Em} L^{p}(\Omega)$ podemos definir a norma usual como segue

$$
\|u\|_{p}=\left(\int_{\Omega}|u(x)|^{p} d x\right)^{1 / p}, \quad 1 \leq p<\infty .
$$

Deste modo, $\left(L^{p}(\Omega) ;\|\cdot\|_{p}\right)$ é um espaço de Banach. No caso particular, quando $p=2$, temos também que $L^{2}(\Omega)$ é um espaço de Hilbert com produto escalar $(u, v)=\int_{\Omega} u(x) v(x) d x$ e norma $\|u\|_{2}=(u, u)^{1 / 2}$.

Para $p=\infty$, definimos $L^{\infty}(\Omega)$ como sendo o conjunto (das classes) de funções Lebesgue mensuráveis $u$, limitadas quase sempre (q.s.) sobre $\Omega$. Em termos de conjuntos,

$$
L^{\infty}(\Omega)=\{u: \Omega \rightarrow \mathbb{R} \mid u \text { é mensurável, }|u(x)| \leq K \text { q.s. em } \Omega\} .
$$

Neste caso, diz-se que o número real $K$ é um majorante essencial de $u$ e denotando por $A=$ $\{K \in \mathbb{R}|| u(x) \mid \leq K$ q.s. em $\Omega\}$, podemos definir uma norma em $L^{\infty}(\Omega)$ como

$$
\|u\|_{\infty}=\sup _{x \in \Omega} \operatorname{ess}|u(x)|=\inf A .
$$

Deste modo, $\left(L^{\infty}(\Omega) ;\|\cdot\|_{\infty}\right)$ é um Espaço de Banach.

Dado um multi-índice $\alpha=\left(\alpha_{1}, \ldots, \alpha_{N}\right) \in \mathbb{N}^{N}$ e um ponto $x=\left(x_{1}, \ldots, x_{N}\right) \in \mathbb{R}^{N}$ defini-se

$$
|\alpha|=\alpha_{1}+\alpha_{2}+\cdots+\alpha_{N}, \quad x^{\alpha}=x_{1}^{\alpha_{1}} x_{2}^{\alpha_{2}} \cdots x_{N}^{\alpha_{N}} \quad \text { e } \quad \alpha !=\alpha_{1} ! \alpha_{2} ! \cdots \alpha_{N} !
$$

O operador de Derivação de ordem $\alpha$, denotado por $D^{\alpha}$, é definido como

$$
D^{\alpha} u=\left\{\begin{array}{ccc}
\frac{\partial^{|\alpha|} u}{\partial x_{1}^{\alpha_{1}} \partial x_{2}^{\alpha_{2}} \ldots \partial x_{N}^{\alpha_{N}}} & \text { se } & \alpha \neq(0, \ldots, 0), \\
u & \text { se } & \alpha=(0, \ldots, 0) .
\end{array}\right.
$$

Quando o multi-índice é da forma $\alpha=(0, \ldots, 0, i, 0, \ldots, 0) \in \mathbb{N}^{N}$, o operador derivação $D^{\alpha}$ também é representado pelas seguintes notações

$$
D^{i}=D_{i}=\partial_{x_{i}}=\frac{\partial}{\partial x_{i}}=(\cdot)_{x_{i}}, \quad i=1, \ldots, N .
$$

Agora, para $k=0,1,2, \ldots$, denotamos por $C^{k}(\Omega)$ o conjunto das funções $k$-vezes continuamente diferenciável sobre o aberto $\Omega$. Quando $k=0$ dizemos simplesmente que $C^{0}(\Omega)$ é o conjunto das funções contínuas sobre $\Omega$ e, também, o denotaremos por $C(\Omega)$. Quando $k=\infty$, diremos que $C^{\infty}(\Omega)$ é o conjunto das funções infinitamente diferenciáveis sobre $\Omega$. Para uma função $u \in C^{k}(\Omega)$, defini-se o suporte de $u \operatorname{comosupp}(u)=\overline{\{x \in \Omega ; u(x) \neq 0\}}^{\Omega} \subset \Omega$ e por $C_{0}^{k}(\Omega)$ denotamos as funções $u$ de $C^{k}(\Omega)$ cujo suporte é compacto em $\mathbb{R}^{N}$. Em termos de conjuntos,

$$
\begin{aligned}
C(\Omega) & =\{u: \Omega \rightarrow \mathbb{R} \mid u \text { é contínua }\}, \\
C^{k}(\Omega) & =\{u: \Omega \rightarrow \mathbb{R} \mid u \text { é } k \text {-vezes continuamente diferenciável }\}, \\
C^{\infty}(\Omega) & =\{u: \Omega \rightarrow \mathbb{R} \mid u \text { é infinitamente diferenciável }\}, \\
C_{0}^{k}(\Omega) & =\left\{u \in C^{k}(\Omega) \mid \operatorname{supp}(u) \subset \Omega \text { é compacto }\right\}, \quad k \in \mathbb{N} \text { ou } k=\infty .
\end{aligned}
$$


Definição 1.1.1. Diremos que uma sequência (ou sucessão) de funções $\left\{\varphi_{n}\right\} \subset C_{0}^{\infty}(\Omega)$ converge à uma função $\varphi \in C_{0}^{\infty}(\Omega)$ se, e somente se, existe um subconjunto compacto $K \subset \Omega$ tal que

(i) $\operatorname{supp}(\varphi) \subset K \operatorname{esupp}\left(\varphi_{n}\right) \subset K, \forall n \in \mathbb{N}$.

(ii) $D^{\alpha} \varphi_{n} \rightarrow D^{\alpha} \varphi$ uniformemente sobre $K, \forall \alpha \in \mathbb{N}^{N}$.

Com esta noção de convergência nos espaços $C_{0}^{\infty}(\Omega)$, o denotaremos também por $\mathcal{D}(\Omega)$ e o chamaremos de espaço das funções teste. Além disso, denotando por $\mathcal{L}(X, \mathbb{R})$ o espaço vetorial dos funcionais lineares e contínuos de $X$ em $\mathbb{R}$, definimos o espaço das distribuições sobre $\Omega$ com valores em $\mathbb{R}$ por

$$
\mathcal{D}^{\prime}(\Omega):=\mathcal{L}(\mathcal{D}(\Omega), \mathbb{R}),
$$

onde a continuidade é entendida no sentido da convergência em $\mathcal{D}(\Omega)$.

Com relação à derivada de uma distribuição a valores reais, lembramos que para $f \in \mathcal{D}^{\prime}(\Omega)$, sua derivada de ordem $\alpha \in \mathbb{N}^{N}$ é dada por

$$
\left\langle D^{\alpha} f, \varphi\right\rangle=(-1)^{|\alpha|}\left\langle f, D^{\alpha} \varphi\right\rangle, \quad \forall \varphi \in \mathcal{D}(\Omega)
$$

A seguir vamos relembrar a definição dos espaços de Sobolev, os quais constituirão fundamental importância em nossas considerações futuras. Para $m \in \mathbb{N}$ e $1 \leq p \leq \infty$, denotamos por $W^{m, p}(\Omega)$ o conjunto (das classes) de funções $u$ em $L^{p}(\Omega)$, tais que suas derivadas $D^{\alpha} u, 0 \leq|\alpha| \leq m$, ainda pertençam a $L^{p}(\Omega)$, onde a derivada se dá no sentido das distribuições. Em termos de conjuntos,

$$
W^{m, p}(\Omega)=\left\{u \in L^{p}(\Omega)\left|D^{\alpha} u \in L^{p}(\Omega), \quad 0 \leq\right| \alpha \mid \leq m\right\} .
$$

Em $W^{m, p}(\Omega)$ tem-se bem definida uma norma dada por

$$
\|u\|_{m, p}=\left(\sum_{0 \leq|\alpha| \leq m}\left\|D^{\alpha} u\right\|_{p}^{p}\right)^{1 / p}, \quad 1 \leq p<\infty
$$

e

$$
\|u\|_{m, \infty}=\max _{0 \leq|\alpha| \leq m}\left\|D^{\alpha} u\right\|_{\infty} .
$$

Com isto, $\left(W^{m, p}(\Omega),\|\cdot\|_{m, p}\right)$ é um espaço de Banach. Além disso, definimos o conjunto $W_{0}^{m, p}(\Omega)$ como o subespaço de $W^{m, p}(\Omega)$ constituido pelo fecho de $C_{0}^{\infty}(\Omega)$ em $W^{m, p}(\Omega)$, ou seja,

$$
W_{0}^{m, p}(\Omega)={\overline{C_{0}^{\infty}(\Omega)}}^{W^{m, p}(\Omega)} .
$$

No caso particular $p=2$, o espaço $W^{m, 2}(\Omega)\left(W_{0}^{m, 2}(\Omega)\right)$ é também um espaço de Hilbert com o correspondente produto interno e, usualmente, é denotado por $H^{m}(\Omega)\left(H_{0}^{m}(\Omega)\right)$.

No que segue, $X$ denotará um espaço de Banach com norma $\|\cdot\|_{X}$ e $H$ denotará um espaço de Hilbert com produto interno $(\cdot, \cdot)_{H}$ e norma $\|\cdot\|_{H}$. 
No problema que iremos estudar no Capítulo 3 deste trabalho faz-se necessário o uso de espaços com peso $L_{\mu}^{2}$. Por este motivo, vamos introduzi-los neste momento para clarear as ideias. Dada uma função "peso"

$$
\mu \in C^{1}\left(\mathbb{R}^{+}\right) \cap L^{1}\left(\mathbb{R}^{+}\right),
$$

definimos o seguinte espaço de Hilbert.

$$
L_{\mu}^{2}\left(\mathbb{R}^{+} ; H\right)=\left\{\xi: \mathbb{R}^{+} \rightarrow H \mid \int_{0}^{\infty} \mu(s)\|\xi(s)\|_{H}^{2} d s<\infty\right\},
$$

munido do produto interno

$$
\left(\xi_{1}, \xi_{2}\right)_{\mu, H}=\int_{0}^{\infty} \mu(s)\left(\xi_{1}(s), \xi_{2}(s)\right)_{H} d s, \quad \forall \xi_{1}, \xi_{2} \in L_{\mu}^{2}\left(\mathbb{R}^{+} ; H\right)
$$

e norma

$$
\|\xi\|_{\mu, H}^{2}=\int_{0}^{\infty} \mu(s)\|\xi(s)\|_{H}^{2} d s, \quad \forall \xi \in L_{\mu}^{2}\left(\mathbb{R}^{+} ; H\right) .
$$

Observação 1.1.1. Somente para título de informação, notamos ao leitor que toda teoria construida para espaços de Hilbert pode ser aplicada aos espaços $L^{2}(\Omega), W^{m, 2}(\Omega)$ e $L_{\mu}^{2}\left(\mathbb{R}^{+} ; H\right)$, bem como a teoria para espaços de Banach pode ser aplicada, de um modo mais geral, aos espaços $L^{p}(\Omega), W^{m, p}(\Omega)$ e $L_{\mu}^{2}\left(\mathbb{R}^{+} ; X\right)$.

Terminaremos esta primeira seção apresentando os espaços de Banach (ou Hilbert) a valores abstratos $L^{p}(a, b ; X), C^{k}([a, b], X), C_{w}([a, b], X)$ e o espaços da distribuições vetoriais $\mathcal{D}^{\prime}(a, b ; X)$, para $1 \leq p \leq \infty,-\infty \leq a<b \leq+\infty$ e $k \in \mathbb{N}$. Contudo, para nossos propósitos futuros é suficiente compreender os espaços acima com $a=0$ e $b=T$, onde $T>0$ é um número real positivo fixado ou $T=\infty$.

Para $1 \leq p<\infty$, representaremos por $L^{p}(0, T ; X)$ o conjunto (das classes) de funções vetoriais mensuráveis $u:(0, T) \rightarrow X$, tais que $\|u(t)\|_{X}$ pertence a $L^{p}(0, T)$. Em termos de conjunto,

$$
L^{p}(0, T ; X)=\left\{u:(0, T) \rightarrow X \mid u \text { é mensurável, } \int_{0}^{T}\|u(t)\|_{X}^{p} d t<\infty\right\} .
$$

O espaço $L^{p}(0, T ; X)$ munido da norma

$$
\|u\|_{L^{p}(0, T ; X)}=\left(\int_{0}^{T}\|u(t)\|_{X}^{p} d t\right)^{1 / p}
$$

torna-se um espaço de Banach.

Quando $p=\infty$, representaremos por $L^{\infty}(0, T ; X)$ o conjunto (das classes) de funções vetoriais mensuráveis $u:(0, T) \longrightarrow X$, tais que $\|u(t)\|_{X}$ pertence a $L^{\infty}(0, T)$. Em termos de conjunto,

$$
L^{\infty}(0, T ; X)=\left\{u:(0, T) \rightarrow X \mid u \text { é mensurável, }\|u(t)\|_{X} \leq K \text { q.s. em }(0, T)\right\} .
$$

Em $L^{\infty}(0, T ; X)$ defini-se uma norma dada por

$$
\|u\|_{L^{\infty}(0, T ; X)}=\quad \operatorname{supess}_{t \in(0, T)}\|u(t)\|_{X}
$$

o que o torna também um espaço de Banach.

Além disso, é fácil ver que valem as seguintes propriedades: 
a) Se $X \hookrightarrow Y$, então $L^{p}(0, T ; X) \hookrightarrow L^{p}(0, T ; Y), \quad 1 \leq p \leq \infty$.

b) $L^{\infty}(0, T ; X) \stackrel{T<\infty}{\hookrightarrow} L^{p}(0, T ; X) \hookrightarrow L^{1}(0, T ; X), \quad 1<p<\infty$.

Para $k \in \mathbb{N}$, definimos também o espaço de Banach

$C^{k}([0, T], X)=\{u:[0, T] \rightarrow X \mid u$ é $k$-vezes continuamente diferenciável de $[0, T]$ em $X\}$,

munido da norma

$$
\|u\|_{C^{k}([0, T], X)}=\sum_{j=0}^{k} \max _{t \in[0, T]}\left\|\frac{d^{j} u(t)}{d t^{j}}\right\|_{X} .
$$

Para abrangir todos os espaços que surgirão nos capítulos seguintes, lembraremos também o conceito continuidade fraca de funções a valores abstratos. Denotaremos por $C_{w}([0, T], X)$ o espaço de todas as funções $u:[0, T] \rightarrow X$ que é contínua de $[0, T]$ em $(X, \tau)$, onde $\tau=\sigma\left(X, X^{\prime}\right)$ designa a topologia fraca de $X$, ou seja, para toda $\varphi \in X^{\prime}$ (dual de $X$ ) a função $t \mapsto\langle u(t), \varphi\rangle$ é contínua em $[0, T]$. Em termos de conjunto temos

$$
C_{w}([0, T], X)=\{u:[0, T] \rightarrow X \mid u \text { é contínua de }[0, T] \text { em }(X, \tau)\} .
$$

Um pouco mais adiante, em uma subseção saparada, faremos uma breve revisão sobre do conceito de topologia fraca para que a definição acima não fique "dependurada" no texto. Antes disso, vamos expor também o espaço das distribuições a valores abstratos.

Denotando por $\mathcal{L}(X, Y)$ o espaço vetorial das transformações lineares e contínuas de $X$ em $Y$, definimos o espaço das distribuições vetoriais de $(0, T)$ com valores em $X$ por

$$
\mathcal{D}^{\prime}(0, T ; X):=\mathcal{L}(\mathcal{D}(0, T), X)
$$

Para $f \in \mathcal{D}^{\prime}(0, T ; X)$, lembramos ainda que sua derivada de ordem $n$ no sentido das distribuições vetoriais é definida por

$$
\left\langle\frac{d^{n} f}{d t^{n}}, \varphi\right\rangle=(-1)^{n}\left\langle f, \frac{d^{n} \varphi}{d t^{n}}\right\rangle, \quad \forall \varphi \in \mathcal{D}(0, T)
$$

Além disso, se $f$ é derivável no sentido das distribuições vetoriais, então podemos enxergar $\frac{d f}{d t}$ como um elemento de $\mathcal{D}^{\prime}(0, T ; X)$, valendo a relação (1.1).

Seguindo as ideais introduzidas em $[66,75]$, vamos estabelecer uma conexão entre os espaços definidos acima. De fato, dada $f \in L^{p}(0, T ; X)$, então pode-se identificar $f$ com uma distribuição vetorial (que aqui ainda denotaremos por $f$ ), de modo que

$$
\langle f, \varphi\rangle=\int_{0}^{T} f(t) \varphi(t) d t, \quad \forall \varphi \in \mathcal{D}(0, T) .
$$

onde a integral é entendida no sentido de Bochner. Com isto, podemos dizer com um certo abuso de notação que

$$
L^{p}(0, T ; X) \subset \mathcal{D}^{\prime}(0, T ; X) .
$$




\subsubsection{Um breve repasso ao conceito de topologia fraca e fraca estrela}

Vamos estabelecer a seguir os conceitos de convergência fraca e fraca estrela num espaço de Banach $X$ com norma $\|\cdot\|_{X}$. Consideremos inicialmente o dual topológico $X^{\prime}=\mathcal{L}(X, \mathbb{R})$, que é também um espaço de Banach quando munido da norma

$$
\|f\|_{X^{\prime}}=\sup _{x \in X,\|x\|_{X} \leq 1}|\langle f, x\rangle| .
$$

Além disso, podemos considerar o espaço de Banach Bidual $X^{\prime \prime}=\mathcal{L}\left(X^{\prime}, \mathbb{R}\right)$ de $X$, munido da norma

$$
\|\xi\|_{X^{\prime \prime}}=\sup _{f \in X^{\prime},\|f\|_{X^{\prime}} \leq 1}|\langle\xi, f\rangle| .
$$

Como é bem sabido da teoria de análise funcional (ver por exemplo Brézis [15]), a aplicação

$$
\begin{aligned}
J: X & \longrightarrow X^{\prime \prime} \\
x & \longmapsto J_{x}: X^{\prime} \longrightarrow \mathbb{R} \\
& \quad f \longmapsto J_{x}(f)=\langle f, x\rangle,
\end{aligned}
$$

é um isomorfismo de $X$ sobre $J(X)$. Isto nos permite identificar $X$ com $J(X) \subset X^{\prime \prime}$.

Agora, para cada $f \in X^{\prime}$ consideremos o funcional

$$
\begin{aligned}
\varphi_{f}: X & \longrightarrow \mathbb{R} \\
x & \longmapsto \varphi_{f}(x)=\langle f, x\rangle,
\end{aligned}
$$

Assim, percorrendo $f$ em $X^{\prime}$ obteremos uma família de aplicações $\left\{\varphi_{f}\right\}_{f \in X^{\prime}}$. Sob estas considerações dizemos que:

(i) A topologia fraca $\sigma\left(X, X^{\prime}\right)$ em $X$ é a topologia mais grossa (menos fina) em $X$ no qual são contínuas todas funções $\varphi_{f}, f \in X^{\prime}$.

(ii) A topologia fraca estrela $\sigma\left(X^{\prime}, X\right)$ em $X^{\prime}$ é a topologia mais grossa (menos fina) em $X^{\prime}$ no qual são contínuas todas funções $J_{x}, x \in X$.

Agora estamos aptos a definir os conceitos de convergência mencionados acima.

Definição 1.1.2. Diremos que uma sequência $\left\{x_{n}\right\}_{n \in \mathbb{N}} \subset X$ converge fraco para $x \in X$, quando $\left\{x_{n}\right\}$ converge a $x$ na topologia fraca $\sigma\left(X, X^{\prime}\right)$, isto é, para todo funcional $f \in X^{\prime}$ temos

$$
\left\langle f, x_{n}\right\rangle \rightarrow\langle f, x\rangle .
$$

Denotaremos a convergência fraca de $\left\{x_{n}\right\}$ a $x$ por $x_{n} \rightarrow x$.

Definição 1.1.3. Diremos que uma sequência $\left\{f_{n}\right\}_{n \in \mathbb{N}} \subset X^{\prime}$ converge fraco estrela para $f \in X^{\prime}$, quando $\left\{f_{n}\right\}$ converge a $f$ na topologia fraca estrela $\sigma\left(X^{\prime}, X\right)$, isto é, para todo $x \in X$ temos

$$
\left\langle f_{n}, x\right\rangle \rightarrow\langle f, x\rangle \text {. }
$$

Denotaremos a convergência fraca estrela de $\left\{f_{n}\right\}$ a $f$ por $f_{n} \stackrel{*}{\rightarrow} f$. 
Observação 1.1.2. Como é usual a convergência forte (em norma) de $\left\{x_{n}\right\}$ a $x \in X$ será denotada por $x_{n} \rightarrow x$.

Aproveitando as considerações anteriores vamos apresentar os importantes conceitos de reflexividade e separabilidade.

Definição 1.1.4. Um espaço de Banach $X$ é chamado reflexivo quando $J(X)=X^{\prime \prime}$, onde a aplicação $J$ é dada em (1.2). O espaço $X$ é chamado separável quando existe um subconjunto enumerável e denso $Y \subset X$.

Observação 1.1.3. Com relação aos espaços mencionados na seção 1.1, valem as seguintes identificações para $1 \leq p<\infty, q$ satisfazendo $1 / p+1 / q=1$ e $m \geq 1$,

$$
\begin{aligned}
{\left[L^{p}(\Omega)\right]^{\prime} } & \cong L^{q}(\Omega) \quad \text { e } \quad\left[L^{\infty}(\Omega)\right]^{\prime} \nsupseteq L^{1}(\Omega), \\
{\left[W_{0}^{m, p}(\Omega)\right]^{\prime} } & \cong W^{-m, q}(\Omega), \\
{\left[L^{p}(0, T, X)\right]^{\prime} } & \cong L^{q}\left(0, T, X^{\prime}\right) .
\end{aligned}
$$

\subsection{Alguns resultados importantes}

Nesta seção colecionaremos alguns dos resultados mais clássicos provenientes da teoria geral de análise funcional e espaços de Sobolev, os quais serão extremamente úteis para estudarmos os problemas propostos nesta tese. Nos resultados abaixo seguimos as notações introduzidas em Adams \& Fournier [1], Brézis [15], Evans [36], Henry [57], Lions [66], Lions \& Magenes [67], Pazy [85] e Zheng [94]. Também usamos as referências em português de Cavalcanti \& Domingos Cavalcanti [17], Medeiros [75], Medeiros \& Milla Miranda [76].

Lema 1.2.1. Seja $\Omega$ um domínio de $\mathbb{R}^{N}$.

(i) Se $1<p<\infty$, então $L^{p}(\Omega)$ é reflexivo. Entretanto, $L^{1}(\Omega)$ e $L^{\infty}(\Omega)$ não são reflexivos.

(ii) Se $1 \leq p<\infty$, então $L^{p}(\Omega)$ é separável. Entretanto, $L^{\infty}(\Omega)$ não é Separável.

Teorema 1.2.2 (Teorema de Densidade). Seja $\Omega$ um domínio de $\mathbb{R}^{N}$.

(i) Se $k \geq 0$, então $C_{0}^{k}(\Omega)$ é denso em $L^{p}(\Omega)$, para $1 \leq p<\infty$.

(ii) Se $\Omega$ é de classe $C^{m}, m \geq 1$, então $C^{m}(\bar{\Omega})$ é denso em $W^{m, p}(\Omega)$, para $1 \leq p<\infty$.

Teorema 1.2.3 (Imersões de Sobolev). Seja $\Omega \subset \mathbb{R}^{N}$ um domínio limitado com fronteira de classe $C^{m}$.

(i) Se $m p<N$, então a seguinte inclusão é contínua

$$
W^{m, p}(\Omega) \hookrightarrow L^{q^{*}}(\Omega), \quad \text { onde } \quad \frac{1}{q^{*}}=\frac{1}{p}-\frac{m}{N} .
$$

Além disso, a inclusão é compacta para qualquer $q$, com $1 \leq q<q^{*}$. 
(ii) Se $m p=N$, então a seguinte inclusão é contínua e compacta

$$
W^{m, p}(\Omega) \hookrightarrow L^{q}(\Omega), \quad \text { para todo } \quad 1 \leq q<\infty
$$

Além disso, se $p=1$ e $m=N$, então vale a mesma relação acima para $q=\infty$.

(iii) Se $k+1>m-\frac{N}{p}>k, k \in \mathbb{N}$, então escrevendo $m-\frac{N}{p}=k+\alpha$, com $0<\alpha<1$, temos que a seguinte inclusão é contínua

$$
W^{m, p}(\Omega) \hookrightarrow C^{k, \alpha}(\bar{\Omega}),
$$

onde $C^{k, \alpha}(\bar{\Omega})$ representa o espaço das funções em $C^{k}(\bar{\Omega})$ cujas derivadas de ordem $k$ são $\alpha$-Hölder contínuas. Além disso, se $N=m-k-1, \alpha=1$ e $p=1$, então a inclusão vale também para $\alpha=1$, e a inclusão $W^{m, p}(\Omega) \hookrightarrow C^{k, \beta}(\bar{\Omega})$ é compacta para todo $0 \leq \beta<\alpha$.

Observação 1.2.1. Para uma versão que estabelece imersões compactas no caso $N$-dimensional veja também o Teorema de Rellich-Kondrachov em Adams \& Furnier [1, Capítulo 6].

Teorema 1.2.4 (Desigualdade de Gagliardo-Nirenberg). Seja $\Omega \subset \mathbb{R}^{N}$ um domínio limitado com fronteira de classe $C^{m}$ e $u \in W^{m, r}(\Omega) \cap L^{q}(\Omega)$ onde $1 \leq r, q \leq \infty$. Para qualquer inteiro $j$ com $0 \leq j<m$ e qualquer $\theta$ com $j / m \leq \theta \leq 1$, temos

$$
\left\|D^{j} u\right\|_{p} \leq C\|u\|_{m, r}^{\theta}\|u\|_{q}^{1-\theta}
$$

desde que

$$
\frac{1}{p}=\frac{j}{N}+\theta\left(\frac{1}{r}-\frac{m}{N}\right)+(1-\theta) \frac{1}{q}
$$

$e m-j-N / r$ não é um inteiro não negativo. Se $m-j-N / r$ é um inteiro não negativo, (1.3) vale $\operatorname{com} \theta=j / m$.

Observação 1.2.2. Em Zheng [94, Capítulo 1] o autor também relembra a desigualdade de GagliardoNirenberg de uma forma mais completa (ver Teorema 1.3.4 nesta referência). Uma outra versão clássica da desigualdade de Gagliardo-Nirenberg também pode ser encontrada em Henry [57, Capítulo 1], sob uma forma levemente mais geral. Além disso, usando a desigualdade de GagliardoNirenberg pode-se estabelecer também resultados de imersões contínuas envolvendo domínio de operadores com potências fracionárias, ver por exemplo o Teorema 1.6.1 em Henry [57, Capítulo 1] ou Teorema 4.3 em Pazy [85, Capítulo 8].

Teorema 1.2.5 (Teorema de Interpolação). Seja $\Omega \subset \mathbb{R}^{N}$ um domínio limitado com fronteira suave. Suponhamos que

$$
\begin{array}{rrr}
p \leq q \leq \infty & \text { se } & m p>N \\
p \leq q<\infty & \text { se } & m p=N \\
p \leq q \leq \frac{N p}{N-m p} & \text { se } & m p<N
\end{array}
$$


Então existe uma constante $K=K(N, m, p, q, \Omega)>0$ tal que para toda $u \in W^{m, p}(\Omega)$,

$$
\|u\|_{q} \leq K\|u\|_{m, p}^{\theta}\|u\|_{p}^{1-\theta}
$$

onde $\theta=(N / m p)-(N / m q)$.

Teorema 1.2.6 (Desigualdade de Poincaré). Seja $\Omega \subset \mathbb{R}^{N}$ um domínio limitado e $1 \leq p<\infty$. Então existe uma constante $C=C(p,|\Omega|)>0$ tal que

$$
\|u\|_{p} \leq C\|\nabla u\|_{p}, \quad \forall u \in W_{0}^{1, p}(\Omega) .
$$

Teorema 1.2.7 (Fórmula de Green). Seja $\Omega \subset \mathbb{R}^{N}$ um aberto limitado com fronteira $\Gamma$ suave. Se $u, v \in H^{2}(\Omega)$, então

$$
-\int_{\Omega}(\Delta u) v d x=\int_{\Omega} \nabla u \cdot \nabla v d x-\int_{\Gamma} \frac{\partial u}{\partial \nu} v d S
$$

onde $\nu$ representa o vetor normal unitário exterior a $\Gamma$ e $\frac{\partial u}{\partial \nu}:=\nabla u \cdot \nu$ a derivada normal de $u$.

Teorema 1.2.8 (Desigualdade de Hölder). Sejam $1 \leq p, q \leq \infty \operatorname{com} \frac{1}{p}+\frac{1}{q}=1 e \Omega \subset \mathbb{R}^{N}$. Se $u \in L^{p}(\Omega)$ e $v \in L^{q}(\Omega)$, então $u v \in L^{1}(\Omega) e$

$$
\int_{\Omega}|u(x) v(x)| d x \leq\|u\|_{p}\|v\|_{q} .
$$

Teorema 1.2.9 (Desigualdade de Hölder Generalizada). Sejam $1 \leq p_{1}, p_{2}, \ldots, p_{n} \leq \infty$ tais que $\frac{1}{p_{1}}+\frac{1}{p_{2}}+\cdots+\frac{1}{p_{n}}=\frac{1}{r} \leq 1$. Se $f_{i} \in L^{p_{i}}(\Omega)$ para $i=1, \ldots, n$, então $f:=\prod_{i=1}^{n} f_{i} \in L^{r}(\Omega) e$

$$
\|f\|_{r} \leq \prod_{i=1}^{n}\left\|f_{i}\right\|_{p_{i}} .
$$

Lema 1.2.10 (Desigualdade de Gronwall). Sejam $\alpha \geq 0$ uma constante e $\phi \in L^{\infty}(a, b), \beta \in$ $L^{1}(a, b)$ tais que $\beta>0, \phi \geq 0$. Se

$$
\phi(t) \leq \alpha+\int_{a}^{b} \beta(s) \phi(s) d s, \quad a \leq t \leq b
$$

então

$$
\phi(t) \leq \alpha e^{\int_{a}^{b} \beta(s) d s}, \quad a \leq t \leq b .
$$

Lema 1.2.11 (Desigualdade de Young). Seja $1<p, q<\infty \operatorname{com} \frac{1}{p}+\frac{1}{q}=1$. Então

$$
a b \leq \frac{a^{p}}{p}+\frac{b^{q}}{q}, \quad \forall a, b \geq 0 .
$$

Lema 1.2.12 (Desigualdade de Young $\operatorname{com} \epsilon$ ). Seja $1<p, q<\infty \operatorname{com} \frac{1}{p}+\frac{1}{q}=1$ e $\epsilon>0$ qualquer. Então

$$
a b \leq \epsilon a^{p}+C_{\epsilon} b^{q}, \quad \forall a, b \geq 0,
$$

onde $C_{\epsilon}=(\epsilon p)^{-q / p} q^{-1}$ 
Observação 1.2.3. No caso particular em que $p=q=2$, a desigualdade de Young com $\epsilon>0$ se resume em

$$
a b \leq \epsilon a^{2}+\frac{1}{4 \epsilon} b^{2}, \quad \forall a, b \geq 0,
$$

sendo também conhecida como desigualdade de Cauchy com $\epsilon$. Esta desigualdade será extremamente utilizada em todos os capítulos seguintes desta tese.

O próximo resultado também é de fundamental importância em nossos problemas futuros.

Teorema 1.2.13 (Aubin-Lions). Sejam $X_{0}, X, X_{1}$ três espaços de Banach com $X_{0}$ e $X_{1}$ reflexivos. Suponhamos que $X_{0} \hookrightarrow \hookrightarrow X \hookrightarrow X_{1}$, e para quaisquer $p_{0}, p_{1}$ com $1<p_{0}, p_{1}<\infty$, consideremos $o$ espaço

$$
W=\left\{u \mid u \in L^{p_{0}}\left(0, T ; X_{0}\right), u_{t} \in L^{p_{1}}\left(0, T ; X_{1}\right)\right\},
$$

munido da norma $\|u\|_{W}=\|u\|_{L^{p_{0}\left(0, T ; X_{0}\right)}}+\left\|u_{t}\right\|_{L^{p_{1}\left(0, T ; X_{1}\right)}}$. Então,

$$
W \hookrightarrow \hookrightarrow L^{p_{0}}(0, T ; X) .
$$

Lema 1.2.14. Seja $X$ um espaço de Banach. Se $f \in L^{p}(0, T ; X)$ e $\frac{d f}{d t} \in L^{p}(0, T ; X)$, então $f \in C([0, T], X)$, a menos de um conjunto de medida nula em $[0, T]$.

Lema 1.2.15. Sejam $X$ e $Y$ espaços de Banach tal que $X \hookrightarrow Y$. Então,

$$
\left[L^{\infty}(0, T ; X) \cap C_{w}([0, T], Y)\right] \subset C_{w}([0, T], X) .
$$

Se a inclusão de $X$ em $Y$ for densa e $X$ for reflexivo, então vale também a inclusão contrária.

Teorema 1.2.16 (Teorema de Representação de Riesz-Fréchet). Seja $\left(H,\|\cdot\|_{H},(\cdot, \cdot)_{H}\right)$ um espaço de Hilbert. Para todo funcional $\varphi \in H^{\prime}$, existe um único $f \in H$ tal que

$$
\langle\varphi, v\rangle_{H^{\prime}, H}=(f, v)_{H}, \quad \forall v \in H .
$$

Além disso, $\|\varphi\|_{H^{\prime}}=\|f\|_{H}$.

Teorema 1.2.17 (Teorema de Lax-Milgram). Seja $\left(H,\|\cdot\|_{H},(\cdot, \cdot)_{H}\right)$ um espaço de Hilbert e $a(u, v)$ uma forma bilinear contínua e coerciva. Então para todo funcional $\varphi \in H^{\prime}$, existe um único $u \in H$ tal que

$$
a(u, v)=\langle\varphi, v\rangle_{H^{\prime}, H}, \quad \forall v \in H .
$$

Além disso, se a $(u, v)$ é simétrica, então u se caracteriza pela propriedade

$$
u \in H \quad e \quad \frac{1}{2} a(u, u)-\langle\varphi, u\rangle_{H^{\prime}, H}=\min _{v \in H}\left\{\frac{1}{2} a(v, v)-\langle\varphi, v\rangle_{H^{\prime}, H}\right\} .
$$

Teorema 1.2.18 (Compacidade Fraca). Seja $X$ um espaço de Banach reflexivo. Se $B \subset X$ é limitado, então $B$ é compacto na topologia fraca $\sigma\left(X, X^{\prime}\right)$, isto é, qualquer sequência $\left\{x_{n}\right\} \subset B$ possui uma subsequência $\left\{x_{n_{k}}\right\}$ convergente em $X$ na topologia fraca $\sigma\left(X, X^{\prime}\right)$.

Teorema 1.2.19 (Compacidade Fraca Estrela). Seja $X$ um espaço de Banach separável. Se $F \subset X^{\prime}$ é limitado, então $F$ é compacto na topologia fraca estrela $\sigma\left(X^{\prime}, X\right)$, isto é, qualquer sequência $\left\{f_{n}\right\} \subset F$ possui uma subsequência $\left\{f_{n_{k}}\right\}$ convergente em $X^{\prime}$ na topologia fraca estrela $\sigma\left(X^{\prime}, X\right)$. 


\subsection{O operador $p$-Laplaciano}

O operador pseudo Laplaciano, que neste trabalho será chamado simplesmente de operador $p$ Laplaciano, e denotado por $\Delta_{p}$, é definido sob duas formas:

$$
\Delta_{p} u=\operatorname{div}\left(|\nabla u|^{p-2} \nabla u\right), \quad \forall u \in W_{0}^{1, p}(\Omega),
$$

ou

$$
\Delta_{p} u=\sum_{j=1}^{N} \frac{\partial}{\partial x_{j}}\left(\left|\frac{\partial u}{\partial x_{j}}\right|^{p-2} \frac{\partial u}{\partial x_{j}}\right), \quad \forall u \in W_{0}^{1, p}(\Omega),
$$

onde $\Omega \subset \mathbb{R}^{N}$ e $p>1$. Estas duas formas assumidas pelo operador $p$-Laplaciano correspondem às derivadas de Fréchet em $W_{0}^{1, p}(\Omega)$ dos funcionais

$$
\frac{1}{p} \int_{\Omega}|\nabla u|^{p} d x \quad \text { e } \quad \frac{1}{p} \sum_{j=1}^{N} \int_{\Omega}\left|\frac{\partial u}{\partial x_{j}}\right|^{p} d x,
$$

respectivamente. Cabe ressaltar que $\Delta_{p} u$ é um operador de segunda ordem não linear, sendo que no caso particular $p=2$ o mesmo se reduz ao operador (linear) Laplaciano $\Delta u=\sum_{j=1}^{N} \frac{\partial^{2} u}{\partial x_{j}^{2}}$. Por questões técnicas vamos utilizar, por todo esse trabalho, a primeira das formas estipuladas para o operador $p$-Laplaciano.

É interessante lembrar também que o operador $p$-Laplaciano aplica $W_{0}^{1, p}(\Omega)$ no espaço $W^{-1, p^{\prime}}(\Omega)=\left[W_{0}^{1, p}(\Omega)\right]^{\prime}$, onde $2 \leq p<\infty$ e $1 / p+1 / p^{\prime}=1$, ou seja,

$$
\begin{aligned}
\Delta_{p}: W_{0}^{1, p}(\Omega) & \longrightarrow W^{-1, p^{\prime}}(\Omega) \\
u & \longmapsto \Delta_{p} u=\operatorname{div}\left(|\nabla u|^{p-2} \nabla u\right) .
\end{aligned}
$$

Observação 1.3.1. Questões relacionadas à monotonicidade, hemicontinuidade e coercividade do operador $p$-Laplaciano podem ser encontradas no livro de Lions [66, Capítulo 2]. Além disso, vale a pena lembrar que $\Delta_{p}$ é um operador limitado, isto é, leva conjuntos limitados de $W_{0}^{1, p}(\Omega)$ em conjuntos limitados de $W^{-1, p^{\prime}}(\Omega)$. A título de exemplo, citamos também o trabalho de Zheng [94, Capítulo 3] para uma abordagem de tais conceitos.

Vamos introduzir agora uma importante condição relacionada ao operador $p$-Laplaciano que usaremos com certa frequência nos dois próximos capítulos desta tese, a saber, a seguinte identidade

$$
-\left\langle\Delta_{p} u, v\right\rangle_{W^{-1, p^{\prime}}(\Omega), W_{0}^{1, p}(\Omega)}=\left\langle|\nabla u|^{p-2} \nabla u, \nabla v\right\rangle_{L^{p^{\prime}}(\Omega), L^{p}(\Omega)}
$$

vale para todos $u, v \in W_{0}^{1, p}(\Omega)$, conforme estabelecido por Lions [66] (ver também Yang [97]).

\subsubsection{Uma desigualdade interessante}

A seguir forneceremos uma desigualdade que será útil ao abordarmos o termo que envolve o operador $p$-Laplaciano em nossas considerações futuras. Além de eficaz, veremos que esta desigualdade segue como consequência da desigualdade do valor médio para aplicações em $\mathbb{R}^{N}$. 
Lema 1.3.1. Existe uma constante $M=M(p, N)>0$ tal que

$$
\left.|| x\right|^{p-2} x-|y|^{p-2} y\left|\leq M\left(|x|^{p-2}+|y|^{p-2}\right)\right| x-y \mid, \quad \forall x, y \in \mathbb{R}^{N},
$$

onde o símbolo $|\cdot|$ denota a norma euclidiana em $\mathbb{R}^{N}$ e $p \geq 2$.

Prova. Definamos inicialmente a seguinte aplicação

$$
\begin{aligned}
F: \mathbb{R}^{N} & \longrightarrow \mathbb{R}^{N} \\
x & \longmapsto F(x)=|x|^{p-2} x, \quad x=\left(x_{1}, \ldots, x_{N}\right) .
\end{aligned}
$$

Note que as funções componentes de $F$, dadas por

$$
F_{j}(x)=|x|^{p-2} x_{j}, \quad j=1, \ldots, N,
$$

são diferenciáveis em $\mathbb{R}^{N}$. De fato, simples cálculos implicam que

$$
\begin{aligned}
& \frac{\partial}{\partial x_{i}} F_{j}(x)=(p-2)|x|^{p-4} x_{i} x_{j} \quad \text { se } \quad i \neq j, \\
& \frac{\partial}{\partial x_{j}} F_{j}(x)=(p-2)|x|^{p-4} x_{j}^{2}+|x|^{p-2} \quad \text { se } \quad i=j,
\end{aligned}
$$

para $i, j=1, \ldots, N$. Assim é claro que $\frac{\partial}{\partial x_{i}} F_{j}(x)$ são contínuas em $\mathbb{R}^{N}-\{\mathbf{0}\}$ e também é simples verificar pela definição que

$$
\frac{\partial}{\partial x_{i}} F_{j}(\mathbf{0})=0=\lim _{x \rightarrow \mathbf{0}} \frac{\partial}{\partial x_{i}} F_{j}(x), \quad \mathbf{0}=(0, \ldots, 0),
$$

para $i, j=1, \ldots, N$. Isto implica que as derivadas parciais das funções componentes $F_{j}$ existem e são contínuas em $\mathbb{R}^{N}$, ou seja, as funções $F_{j}$ são diferenciáveis em $\mathbb{R}^{N}$ e, consequentemente, a aplicação $F$ é também diferenciável em $\mathbb{R}^{N}$. Logo, fica bem definida a aplicação derivada (operador linear)

$$
\begin{aligned}
F^{\prime}: \mathbb{R}^{N} & \longrightarrow \mathcal{L}\left(\mathbb{R}^{N}, \mathbb{R}^{N}\right) \\
x & \longmapsto F^{\prime}(x): \mathbb{R}^{N} \rightarrow \mathbb{R}^{N}
\end{aligned}
$$

Além disso, temos

$$
\left\|F^{\prime}(x)\right\| \leq N^{2}(p-1)|x|^{p-2}, \quad \forall x \in \mathbb{R}^{N},
$$

onde $\|\cdot\|$ menciona a norma no espaço $\mathcal{L}\left(\mathbb{R}^{N}, \mathbb{R}^{N}\right)$. Com efeito, note que

$$
\left\|F^{\prime}(x)\right\|=\sup _{v \in \mathbb{R}^{N},|v| \leq 1}\left|F^{\prime}(x) \cdot v\right|=\sup _{v \in \mathbb{R}^{N},|v| \leq 1}\left|\frac{\partial F}{\partial v}(x)\right|=\sup _{v \in \mathbb{R}^{N},|v| \leq 1}\left|\left(\frac{\partial F_{1}}{\partial v}(x), \ldots, \frac{\partial F_{N}}{\partial v}(x)\right)\right|,
$$

o que implica em

$$
\left\|F^{\prime}(x)\right\| \leq N \sup _{|v| \leq 1} \max _{1 \leq j \leq N}\left|\frac{\partial F_{j}}{\partial v}(x)\right|
$$


Agora, tomando $v=\left(\alpha_{1}, \ldots, \alpha_{N}\right)$ e observando que as funções $F_{j}$ são diferenciáveis em $\mathbb{R}^{N}$ segue que

$$
\frac{\partial F_{j}}{\partial v}(x)=\sum_{i=1}^{N} \frac{\partial F_{j}(x)}{\partial x_{i}} \alpha_{i}, \quad j=1, \ldots, N
$$

Logo,

$$
\left|\frac{\partial F_{j}}{\partial v}(x)\right| \leq \sum_{i=1}^{N}\left|\frac{\partial F_{j}(x)}{\partial x_{i}}\right|\left|\alpha_{i}\right| \leq\left(\sum_{i=1}^{N}\left|\frac{\partial F_{j}(x)}{\partial x_{i}}\right|^{2}\right)^{\frac{1}{2}}\left(\sum_{i=1}^{N}\left|\alpha_{i}\right|^{2}\right)^{\frac{1}{2}} \leq N \max _{1 \leq i \leq N}\left|\frac{\partial F_{j}}{\partial x_{i}}(x)\right||v|,
$$

de onde segue que

$$
\max _{1 \leq j \leq N}\left|\frac{\partial F_{j}}{\partial v}(x)\right| \leq N \max _{1 \leq i, j \leq N}\left|\frac{\partial F_{j}}{\partial x_{i}}(x)\right||v| \leq N(p-1)|x|^{p-2}|v|
$$

ou ainda,

$$
\sup _{|v| \leq 1} \max _{1 \leq j \leq N}\left|\frac{\partial F_{j}}{\partial v}(x)\right| \leq N(p-1)|x|^{p-2} .
$$

Substituindo (1.6) em (1.5), obtemos (1.4) como desejado.

Dados $x, y \in \mathbb{R}^{N}$ considere o segmento $[y, x] \subset \mathbb{R}^{N}$. Para todo $\xi \in[y, x]$ segue de (1.4) que

$$
\left\|F^{\prime}(\xi)\right\| \leq N^{2}(p-1)|\xi|^{p-2} .
$$

Mais ainda, para $\xi \in[y, x]$, existe uma constante $\theta=\theta(x, y) \in[0,1]$ tal que $\xi=(1-\theta) y+\theta x=$ $y+\theta(x-y)$. Logo,

$$
|\xi|^{p-2} \leq(|y|+\theta|x-y|)^{p-2} \leq 2^{p-2}(|y|+|x|)^{p-2} \leq 2^{2(p-2)}\left(|y|^{p-2}+|x|^{p-2}\right) .
$$

Assim,

$$
\left\|F^{\prime}(\xi)\right\| \leq\left(2^{p-2} N\right)^{2}(p-1)\left(|y|^{p-2}+|x|^{p-2}\right), \quad \forall \xi \in[y, x] .
$$

Como $F$ é diferenciável no segmento aberto $(y, x)$, contínua no segmento fechado $[y, x]$ e vale a estimativa (1.7), então a Desigualdade do Valor Médio para aplicações implica que

$$
|F(x)-F(y)| \leq M\left(|x|^{p-2}+|y|^{p-2}\right)|x-y|,
$$

onde $M=\left(2^{p-2} N\right)^{2}(p-1)$. Isto conclui a prova do Lema 1.3.1.

\subsection{Operadores lineares não limitados}

Iniciaremos esta seção fazendo um breve resumo sobre a construção de operadores lineares não limitados associados a uma forma bilinear. Em seguida, relembraremos alguns fatos sobre os operadores com potência fracionária, os quais terão certa relevância em nossas considerações futuras. Para maiores detalhes sobre o assunto recomendamos os trabalhos de Kreyszig [63], Medeiros \& Milla Miranda [76], Temam [90, 91], Yosida [93] e Zheng [94]. 


\subsubsection{Operador $A$ associado à uma forma bilinear}

Sejam $\left(V,\|\cdot\|_{V},(\cdot, \cdot)_{V}\right)$ e $\left(H,\|\cdot\|_{H},(\cdot, \cdot)_{H}\right)$ dois espaços de Hilbert tais que $V$ é denso em $H$, com inclusão $V \hookrightarrow H$ contínua e compacta. Denotaremos por $V^{\prime}$ o dual de $V$ e por $\langle\cdot, \cdot\rangle$ a dualidade entre $V^{\prime}$ e $V$. Identificando $H$ com seu dual, por meio do Teorema da representação de Riez, obtemos a seguinte cadeia de inclusões

$$
V \hookrightarrow H \cong H^{\prime} \hookrightarrow V^{\prime}
$$

Considerando uma forma bilinear e contínua $a(\cdot, \cdot): V \times V \rightarrow \mathbb{R}$, podemos definir um operador linear $A: V \rightarrow V^{\prime}$ dado por

$$
\langle A u, v\rangle=a(u, v), \quad \forall u, v \in V .
$$

Mais ainda, o domínio do operador $A$ é definido como

$$
D(A)=\{u \in V \mid A u \in H\},
$$

e dizemos ainda que o operador linear $A$ é definido pela terna $\{V, H, a(\cdot, \cdot)\}$.

Relembrando, temos da teoria de análise funcional (ver Temam [91, Capítulo 2]) que se $a(\cdot, \cdot)$ for uma forma bilinear contínua, coerciva e simétrica, então o operador linear $A: D(A) \subset H \rightarrow H$ é fechado, não limitado, positivo definido, autoadjunto e uma bijeção (isomorfismo). Além disso, dotando o domínio $D(A)$ com a norma $\|u\|_{D(A)}=\|A u\|_{H}$ (que é equivalente a norma do gráfico $\|u\|_{G}^{2}=\|u\|_{H}^{2}+\|A u\|_{H}^{2}$ ) obtemos que $D(A)$ é um espaço de Hilbert denso em $H$.

Observação 1.4.1. Um exemplo clássico de uma forma bilinear satisfazendo as condições acima é dada pelo produto interno $(\cdot, \cdot)_{V}$ em $V$. Neste caso, considerando o operador $A$ dado pela terna $\left\{V, H,(\cdot, \cdot)_{V}\right\}$, então $A: D(A) \subset H \rightarrow H$ é tal que

$$
(A u, v)_{H}=(u, v)_{V}, \quad \forall u \in D(A), \forall v \in V .
$$

Mediante às condições satisfeitas pelo operador $A$ acima e usando também que a inclusão $V \hookrightarrow H$ é compacta, segue da teoria espectral (ver por exemplo Yosida [93]) que existe uma base ortonormal completa $\left\{w_{j}\right\}_{j \in \mathbb{N}}$ de $H$ e uma sequência de números reais $\left\{\lambda_{j}\right\}_{j \in \mathbb{N}}$ tais que

$$
\begin{aligned}
& 0<\lambda_{1} \leq \lambda_{2} \leq \cdots \quad \text { com } \quad \lambda_{j} \rightarrow \infty \text { quando } j \rightarrow \infty, \\
& w_{j} \in D(A) \quad \text { e } \quad A w_{j}=\lambda_{j} w_{j}, \quad \forall j \in \mathbb{N} .
\end{aligned}
$$

Note que vale ainda as seguintes relações

$$
\left(w_{i}, w_{j}\right)_{H}=\delta_{i j} \quad \text { e } \quad a\left(w_{i}, w_{j}\right)=\lambda_{i} \delta_{i j}, \quad \forall i, j \in \mathbb{N},
$$

onde $\delta_{i j}$ denota o delta de Kronecker. 


\subsubsection{Potências fracionárias do operador $A$}

Sob as hipóteses da subseção anterior, além da base acima mencionada, podemos definir também os operadores com potências fracionárias $A^{s}, s \in \mathbb{R}$, do operador $A$. Mais ainda, os operadores $A^{s}$ podem ser caracterizados em termos da base $\left\{w_{j}\right\}_{j \in \mathbb{N}}$.

Iniciamos notando que para $s>0$ o operador $A^{s}: D\left(A^{s}\right) \subset H \rightarrow H$ é um operador linear não limitado, positivo definido, autoadjunto e injetivo, cujo domínio $D\left(A^{s}\right)$ é denso em $H$. Além disso, munindo $D\left(A^{s}\right)$ com o produto interno e norma

$$
(u, v)_{D\left(A^{s}\right)}=\left(A^{s} u, A^{s} v\right)_{H} \quad \text { e } \quad\|u\|_{D\left(A^{s}\right)}=\left\|A^{s} u\right\|_{H}
$$

obtemos que $D\left(A^{s}\right)$ é um espaço de Hilbert. Mais ainda, $D\left(A^{-s}\right)$ é definido como sendo o dual de $D\left(A^{s}\right)$ e com isto o operador $A^{s}$ pode ser estendido como um isomorfismo de $H$ em $D\left(A^{-s}\right)$. Em $D\left(A^{-s}\right)$ consideramos o produto interno e norma como acima substituindo $s$ por $-s$.

Usando novamente que a inclusão $V \hookrightarrow H$ é compacta, pode-se definir $A^{s}$, para $s>0$, em termos da base $\left\{w_{j}\right\}_{j \in \mathbb{N}}$ por

$$
A^{s} u=\sum_{j=1}^{\infty} \lambda_{j}^{s}\left(u, w_{j}\right)_{H} w_{j}, \quad \forall u \in D\left(A^{s}\right),
$$

onde

$$
D\left(A^{s}\right)=\left\{\left.u \in H\left|\sum_{j=1}^{\infty} \lambda_{j}^{2 s}\right|\left(u, w_{j}\right)_{H}\right|^{2}<\infty\right\} .
$$

Neste caso, a norma em $D\left(A^{s}\right)$ pode ser reescrita como

$$
\|u\|_{D\left(A^{s}\right)}=\left(\sum_{j=1}^{\infty} \lambda_{j}^{2 s}\left|\left(u, w_{j}\right)_{H}\right|^{2}\right)^{1 / 2}, \quad \forall u \in D\left(A^{s}\right) .
$$

Ademais, $D\left(A^{-s}\right)$ é o complemento de $H$ para a norma $\left(\sum_{j=1}^{\infty} \lambda_{j}^{2 s}\left|\left(u, w_{j}\right)_{H}\right|^{2}\right)^{1 / 2}$ e $A^{-s}$ é definido como acima com $-s$ no lugar de $s$.

Um resultado importante para operadores com potência fracionária é o seguinte:

Lema 1.4.1. Se $\alpha \geq 0$ e $\beta>0$, então $D\left(A^{\alpha+\beta}\right) \hookrightarrow \hookrightarrow D\left(A^{\alpha}\right)$.

\subsection{Uma breve revisão sobre semigrupos lineares}

Nesta seção relembraremos alguns conceitos e resultados provenientes da teoria geral de semigrupos lineares. Iniciaremos com várias definições gerais e, em seguida, daremos os resultados que nos interessam ao longo desta tese, os quais serão utilizados com certa frequência no Capítulo 4. Tais resultados serão esboçados conforme os trabalhos de Brézis [15], Liu \& Zheng [70], Pazy [85] e Zheng [94]. Para uma referência em português, ver também Muñoz Rivera [79]. 


\subsection{1 $C_{0}$-semigrupos de operadores lineares}

Nesta seção $\left(X,\|\cdot\|_{X}\right)$ sempre mencionará um espaço de Banach, $\left(H,\|\cdot\|_{H},(\cdot, \cdot)_{H}\right)$ um espaço de Hilbert e $(\mathcal{L}(X, X),\|\cdot\|)$ o espaço dos operadores lineares e contínuos em $X$.

Definição 1.5.1. Uma família $\{T(t)\}_{t \geq 0}$ de operadores lineares e limitados definida sobre um espaço de Banach $X$ é chamada de semigrupo de operadores lineares limitados (ou simplesmente semigrupo) quando

(i) $T(0)=I: X \rightarrow X$ (Operador Identidade em $X)$.

(ii) $T(t+s)=T(t) T(s)$, para cada $t, s \geq 0$.

Ademais, dizemos que $\{T(t)\}_{t \geq 0}$ é um semigrupo de classe $C_{0}$ (ou simplesmente $C_{0}$-semigrupo) se além dos itens acima tivermos que

(iii) $\lim _{t \rightarrow 0} T(t) x=x$, para todo $x \in X$.

Definição 1.5.2. Um operador $A$ é chamado de gerador infinitesimal de um semigrupo $\{T(t)\}_{t \geq 0}$ quando $A$ é definido como

$$
D(A)=\left\{x \in X \mid \lim _{t \rightarrow 0^{+}} \frac{T(t) x-x}{t} \text { existe }\right\}
$$

e para cada $x \in D(A)$ temos

$$
A x=\lim _{t \rightarrow 0^{+}} \frac{T(t) x-x}{t} .
$$

As vezes diz-se também que o semigrupo $T(t)$ é gerado por $A$. Usaremos com frequência a seguinte notação $T(t)=e^{A t}$. Note também que o domínio $D(A)$ do operador $A$ pode ser reescrito como

$$
D(A)=\{x \in X \mid A x \in X\} .
$$

Definição 1.5.3. Um semigrupo $\{T(t)\}_{t \geq 0}$ é chamado de uniformemente limitado se existe uma constante $M \geq 1$ tal que

$$
\|T(t)\| \leq M, \quad \forall t \geq 0 .
$$

Quando $M=1$, diremos também que $\{T(t)\}_{t \geq 0}$ é um semigrupo de contrações .

Definição 1.5.4. Um semigrupo $\{T(t)\}_{t \geq 0}$ é chamado exponencialmente estável se existem constantes $\alpha>0$ e $M \geq 1$ tais que

$$
\|T(t)\| \leq M e^{-\alpha t}, \quad \forall t \geq 0 .
$$

Definição 1.5.5. Seja $R=\left\{z:=r e^{i \theta} \in \mathbb{C} \mid \theta_{1}<\theta<\theta_{2}, \theta_{1}<0<\theta_{2}\right\}$. Um família de operadores lineares limitados $\{T(z)\}_{z \in R}$ definida em $R$ é chamada de semigrupo analítico sobre $R$ quando

(i) $z \mapsto T(z)$ é analítica em $R$. 
(ii) $T(0)=I$ e $\lim _{z \in R, z \rightarrow 0} T(z) x=x$, para todo $x \in R$.

(iii) $T\left(z_{1}+z_{2}\right)=T\left(z_{1}\right) T\left(z_{2}\right)$, para todos $z_{1}, z_{2} \in R$.

Definição 1.5.6. Um $C_{0}$-semigrupo (real) $T(t):=e^{A t}$ é chamado de analítico se ele possui uma extensão analítica $T(z)$ em algum setor $R$ que contém o eixo real não negativo.

Observação 1.5.1. Pelas definições acima, podemos ver que a restrição de um semigrupo analítico ao eixo real é, em particular, um $C_{0}$-semigrupo. O contrário é mais delicado, no entanto, veremos um resultado mais adiante (ver Liu \& Zheng [70] ou Pazy [85]) que nos permitirá estender um $C_{0^{-}}$ semigrupo à um semigrupo analítico em algum setor $R$ em torno do eixo real não negativo.

Definição 1.5.7. Seja $A$ um operador linear (não necessariamente limitado) definido sobre um espaço de Banach $X$. O conjunto resolvente, denotado por $\rho(A)$, é definido como

$$
\rho(A)=\left\{\lambda \in \mathbb{C} \mid \lambda I-A \text { é invertível e }(\lambda I-A)^{-1} \in \mathcal{L}(X, X)\right\} .
$$

O conjunto $\sigma(A)=\mathbb{C} \backslash \rho(A)$ é chamado de espectro de $A$.

Definição 1.5.8. Seja $A$ um operador linear definido sobre um espaço de Hilbert $H$ com domínio $D(A) \subseteq H$. Dizemos que $A$ é um operador dissipativo quando

$$
\operatorname{Re}(A x, x)_{H} \leq 0, \quad \forall x \in D(A) .
$$

\subsubsection{Caracterização dos geradores infinitesimais de $C_{0}$-semigrupos}

Agora vamos apresentar os resultados relacionados à um gerador infinitesimal de um $C_{0^{-}}$ semigrupo $T(t)=e^{A t}$ definido sobre espaços de Banach ou Hilbert.

Teorema 1.5.1 (Hille-Yosida). Um operador linear (não limitado) A é gerador infinitesimal de um $C_{0}$-semigrupo de contrações $\{T(t)\}_{t \geq 0}$ se, e somente se,

(a) A é fechado e $\overline{D(A)}=X$.

(b) O conjunto resolvente $\rho(A)$ de A contém $\mathbb{R}^{+}$e para cada $\lambda>0$

$$
\left\|(\lambda I-A)^{-1}\right\| \leq \frac{1}{\lambda} .
$$

A seguir veremos o Teorema de Lumer-Phillips que nos fornece a caracterização dos geradores infinitesimais de $C_{0}$-semigrupos de contrações sobre espaços de Hilbert. O mesmo resultado vale em espaços de Banach, porém neste trabalho precisaremos apenas do resultado sobre espaços de Hilbert.

Teorema 1.5.2 (Lumer-Phillips). Seja A um operador linear com domínio $D(A)$ denso em um espaço de Hilbert H. Temos:

(a) Se Aé dissipativo e existe um número $\lambda>0$ tal que $\mathcal{I} m(\lambda I-A)=H$, então A é um gerador infinitesimal de um $C_{0}$-semigrupo de contrações em $H$. 
(b) Se A é o gerador infinitesimal de um $C_{0}$-semigrupo de contrações em $H$, então $\operatorname{Im}(\lambda I-A)=$ $H$ para todo $\lambda>0$ e A é dissipativo.

Uma fundamental consequência do Teorema de Lumer-Phillips que usaremos mais adiante é a seguinte:

Corolário 1.5.3. Seja A um operador linear dissipativo com domínio $D(A)$ denso em um espaço de Hilbert $H$. Se $0 \in \rho(A)$, então A é um gerador infinitesimal de um $C_{0}$-semigrupo de contrações em $H$.

\subsubsection{Analiticidade e estabilidade assintótica de $C_{0}$-semigrupos}

Nesta subseção vamos apresentar alguns resultados que lidam com analiticidade, estabilidade exponencial e que geram decaimento do tipo polinomial de $C_{0}$-semigrupos definidos sobre espaços de Hilbert.

O primeiro resultado estabelece uma condição necessária e suficiente para se obter analiticidade. Tal resultado pode ser encontrado de um modo geral para $C_{0}$-semigrupos uniformemente limitados, como proposto pelo Teorema 5.2 em Pazy [85, Capítulo 2]. Contudo, usaremos uma versão devido a Liu \& Young [69], o qual também pode ser encontrado em Liu \& Zheng [70] (ver Teorema 1.3.3 neste último).

Teorema 1.5.4. Seja $T(t)=e^{A t}$ um $C_{0}$-semigrupo de contrações em um espaço de Hilbert $H$ tal que

$$
i \mathbb{R}:=\{i \beta ; \beta \in \mathbb{R}\} \subseteq \rho(A)
$$

Então, $T(t)$ é analítico se, e somente se,

$$
\overline{\lim }_{|\beta| \rightarrow \infty}\left\|\beta\left(i \beta I_{d}-A\right)^{-1}\right\|<\infty
$$

onde a norma $\|\cdot\|$ é dada no espaço $\mathcal{L}(H, H)$.

O próximo resultado concede estabilidade exponencial para um semigrupo e é devido a Gearhart [45] (ver também Prüss [86]). Observamos que existem duas maneiras equivalentes de se obter estabilidade exponencial para $C_{0}$-semigrupo de contrações em espaços de Hilbert e uma prova dessa equivalência pode ser encontrada em Liu \& Zheng [70], o qual nos dá a seguinte versão que fornece uma condição necessária e suficiente para estabilidade exponencial.

Teorema 1.5.5. Seja $T(t)=e^{A t}$ um $C_{0}$-semigrupo de contrações em um espaço de Hilbert H. Então, $T(t)$ é exponencialmente estável se, e somente se, as seguintes condições se verificam

$$
i \mathbb{R}:=\{i \beta ; \beta \in \mathbb{R}\} \subseteq \rho(A)
$$

$e$

$$
\varlimsup_{|\beta| \rightarrow \infty}\left\|\left(i \beta I_{d}-A\right)^{-1}\right\|<\infty .
$$


Estamos interessados em aplicar esses resultados gerais em problemas de evolução dissipativos, enxergando a solução do sistema por meio de um semigrupo. Contudo, tal semigrupo nem sempre é exponencialmente estável (por exemplo, quando a condição (1.11) do Teorema 1.5.5 não se verifica). Neste caso, devemos procurar outra forma de estabilizar o sistema, como por exemplo determinar decaimento polinomial de soluções. Nos últimos anos vários autores desenvolveram resultados sobre decaimento polinomial de semigrupos. Alguns deles podem ser encontrados em Liu \& Rao [68, Teorema 2.1], Bátkai et al [11] e em Fatori \& Rivera [40, Teorema 5.3].

Neste trabalho usaremos uma versão mais recente devido a Borichev \& Tomilov [14] que nos fornece condições equivalentes para obter decaimento do tipo polinomial para soluções em nossos problemas futuros.

Teorema 1.5.6. Seja $\{T(t)\}_{t \geq 0}$ um $C_{0}$-semigrupo limitado em um espaço de Hilbert $H$ com gerador infinitesimal $A$ tal que $i \mathbb{R} \subset \rho(A)$. Então, para alguma constante fixada $\alpha>0$ as seguintes afirmações são equivalentes:

$$
\begin{aligned}
& \left\|\left(i \beta I_{d}-A\right)^{-1}\right\|=O\left(|\beta|^{\alpha}\right), \quad \beta \rightarrow \infty . \\
& \left\|T(t)(-A)^{-\alpha}\right\|=O\left(t^{-1}\right), \quad t \rightarrow \infty . \\
& \left\|T(t)(-A)^{-\alpha} u\right\|_{H}=o\left(t^{-1}\right), \quad t \rightarrow \infty, u \in H . \\
& \left\|T(t) A^{-1}\right\|=O\left(t^{-1 / \alpha}\right), \quad t \rightarrow \infty . \\
& \left\|T(t) A^{-1} u\right\|_{H}=o\left(t^{-1 / \alpha}\right), \quad t \rightarrow \infty, u \in H .
\end{aligned}
$$

Nas sentenças acima, relembramos que a notação $\|\cdot\|$ menciona norma no espaço $\mathcal{L}(H, H)$.

\subsubsection{Problema de Cauchy abstrato}

Como é bem conhecido, a teoria geral de semigrupos nos permite estudar problemas de valor inicial para equações de evolução abstratas do tipo

$$
\left\{\begin{array}{l}
\frac{d}{d t} U(t)=A U(t), \quad t>0, \\
U(0)=U_{0},
\end{array}\right.
$$

onde $A$ é um operador linear com domínio $D(A) \subset X$, sendo $X$ um espaço de Banach (ou Hilbert).

Definição 1.5.9. Uma solução do problema de Cauchy (1.12) é uma função $U:[0,+\infty) \rightarrow X$ tal que $U(t)$ é contínua para $t \geq 0$, continuamente diferenciável com $U(t) \in D(A)$ para $t>0$ e satisfaz (1.12) em $[0,+\infty)$ quase sempre.

O resultado a seguir é um clássico da literatura e pode ser encontrado em Brézis [15, Capítulo 7], Pazy [85, Capítulo 4] ou Zheng [94, Capítulo 2]. Observamos ainda que em [15, 94] os autores usam uma linguagem de operadores $m$-acretivos com respeito ao operador $A$. 
Teorema 1.5.7. Seja A um gerador infinitesimal de um $C_{0}$-semigrupo de contrações $T(t):=e^{A t}$ em $X$. Se $U_{0} \in D(A)$, então o problema de Cauchy abstrato (1.12) possui uma única solução $U$ em $D(A)$ dada por

$$
U(t)=T(t) U_{0}:=e^{A t} U_{0}, \quad \forall t \geq 0
$$

tal que

$$
U \in C([0,+\infty), D(A)) \cap C^{1}([0,+\infty), X)
$$

Para obter soluções mais regulares introduzimos o seguinte espaço de Banach (ou Hilbert)

$$
D\left(A^{k}\right)=\left\{U \in D\left(A^{k-1}\right) \mid A U \in D(A)^{k-1}\right\}, \quad k \in \mathbb{N},
$$

munido da norma $\|U\|_{D\left(A^{k}\right)}^{2}=\sum_{j=0}^{k}\|U\|_{D\left(A^{j}\right)}^{2}$. Com isto, temos o

Teorema 1.5.8. Sob as hipóteses do Teorema 1.5.7, se $U_{0} \in D\left(A^{k}\right)$ com $k \in \mathbb{N}$, então a solução $U$ do problema (1.12) satisfaz a seguinte condição

$$
U \in \bigcap_{j=0}^{k} C^{k-j}\left([0,+\infty), D\left(A^{j}\right)\right)
$$

\subsection{Uma breve revisão sobre atratores globais}

Nesta seção vamos introduzir alguns conceitos e resultados básicos da teoria de atratores globais para semigrupos de operadores não lineares definidos sobre espaços métricos (completos) em geral. Em particular, tais conceitos também são válidos para espaços de Banach e Hilbert, os quais constituem objetos de nosso interesse. As definições e resultados a seguir se baseiam nos trabalhos de Babin \& Vishiki [8], Ceron \& Lopes [21], Chueshov \& Lasiecka [22, 25], Eden et al [35], Hale [55], Khanmamedov [61], Málek \& Pražák [74], Pata \& Zelik [82] e Temam [91]. Para uma referência em português, recomendamos a apostila do professor Alexandre N. Carvalho [16].

Denotaremos por $(X, d)$ um espaço métrico completo e por $\mathcal{C}(X)$ o conjunto das aplicações contínuas de $X$ em $X$. Porém, cabe ressaltar que os conceitos iniciais apresentados a seguir são cabíveis apenas para espaços métricos.

Definição 1.6.1. Uma família de operadores não necessariamente lineares $\{S(t)\}_{t \geq 0} \subset \mathcal{C}(X)$ é chamada de $C_{0}$-semigrupo não linear (ou simplesmente de $C_{0}$-semigrupo), se as seguintes condições forem satisfeitas:

(i) $S(0)=I: X \rightarrow X$ é o operador identidade.

(ii) $S(t+s)=S(t) S(s)$ para cada $t, s \geq 0$.

(iii) A aplicação $[0, \infty) \times X \ni(t, x) \mapsto S(t)(x) \in X$ é contínua para cada $x \in X$ fixado. 
As vezes o par $(X, S(t))$ é também chamado de sistema dinâmico e $S(t)$ um semigrupo de evolução.

Definição 1.6.2. Seja $S(t): X \rightarrow X, t \geq 0$, um $C_{0}$-semigrupo. Diremos que um subconjunto $\mathcal{A} \subset X$ é invariante (ou positivamente invariante ) pelo semigrupo $S(t)$, quando $S(t) \mathcal{A}=\mathcal{A}$ para todo $t \geq 0($ ou $S(t) \mathcal{A} \subset \mathcal{A})$.

Definição 1.6.3. Seja $S(t): X \rightarrow X, t \geq 0$, um $C_{0}$-semigrupo. Um conjunto fechado e limitado $\mathcal{A} \subset X$ é chamado de atrator global para $S(t)$ quando as seguintes propriedades forem satisfeitas:

(i) $\mathcal{A}$ é um conjunto invariante por $S(t)$.

(ii) $\mathcal{A}$ atrai (uniformemente) qualquer subconjunto limitado de $X$ sob a ação de $S(t)$, ou seja, para qualquer limitado $B \subset X$,

$$
\operatorname{dist}_{H}(S(t) B, \mathcal{A}):=\sup _{x \in S(t) B} \inf _{y \in \mathcal{A}} d(x, y) \rightarrow 0 \text { quando } t \rightarrow+\infty .
$$

onde $\operatorname{dist}_{H}(A, B)$ é chamada de semi-distância de Hausdorff entre os subconjuntos $A, B \subset X$.

Para determinar a existência de atratores globais as seguintes noções constituem fundamental importância.

Definição 1.6.4. Seja $S(t): X \rightarrow X, t \geq 0$, um $C_{0}$-semigrupo. Um conjunto $\mathcal{B} \subset X$ é chamado de conjunto absorvente para $S(t)$ se para qualquer subconjunto limitado $B \subset X$, existe $T_{0}=T_{0}(B) \geq 0$ tal que

$$
S(t) B \subset \mathcal{B}, \quad \forall t \geq T_{0} .
$$

Quando $S(t)$ possui um conjunto absorvente limitado, diremos também que o par $(X, S(t))$ constitui um sistema dinâmico dissipativo.

Definição 1.6.5. Um semigrupo $S(t): X \rightarrow X, t \geq 0$, é chamado assintoticamente suave se para qualquer conjunto limitado e positivamente invariante $B \subset X$, existe um conjunto compacto $K \subset \bar{B}^{X}$ tal que

$$
\operatorname{dist}_{H}(S(t) B, K) \rightarrow 0 \text { quando } t \rightarrow+\infty .
$$

Uma abordagem baseada nos trabalhos $[8,25,55,91]$ nos fornece um rápido resultado envolvendo os conceitos acima citados para espaços de Banach.

Proposição 1.6.1. Seja $(X, S(t))$ um sistema dinâmico dissipativo definido sobre um espaço de Banach $X$. Então, $S(t)$ possui um atrator global compacto $\mathcal{A} \subset X$ se, e somente se, $S(t) e ́$ assintoticamente suave.

Com este resultado, que fornece uma condição necessária e suficiente para existência de atratores globais compactos, vemos que é indispensável buscar critérios para que um $C_{0}$-semigrupo seja assintoticamente suave. É o que faremos a seguir, seguindo as ideias como em [21, 22, 25, 55]. 
Definição 1.6.6. Uma pseudométrica $m: X \times X \rightarrow \mathbb{R}$ definida sobre um espaço de Banach $X$ é chamada de pré-compacta (com respeito a norma de $X$ ) se toda sequência limitada em $X$ possui uma subsequência que é de Cauchy com respeito a $m$.

Proposição 1.6.2. Seja $S(t): X \rightarrow X, t \geq 0$, um $C_{0}$-semigrupo definido sobre um espaço de Banach $X$. Suponhamos que para qualquer conjunto limitado e positivamente invariante $B \subset X$, existam funções $K_{B}(t) \geq 0$ e $Q_{B}(t) \geq 0$ tais que $\lim _{t \rightarrow \infty} Q_{B}(t)=0$, um tempo $T_{B} \geq 0$ e uma pseudométrica pré-compacta $m: X \times X \rightarrow \mathbb{R}$ tais que

$$
\|S(t) x-S(t) y\|_{X} \leq Q_{B}(t)\|x-y\|_{X}+K_{B}(t) m(x, y) ; \quad \forall t \geq T_{B}, \quad \forall x, y \in B .
$$

Então, $S(t)$ é assintoticamente suave.

\subsubsection{Condições suficientes para existência de atratores globais}

A título de exemplo, enfatizaremos mais alguns resultados que nos dão condições suficientes para determinar a existência de atratores globais. Nossa abordagem agora seguirá os clássicos $[8,61,91,94]$. No que segue denotaremos por $\left(X,\|\cdot\|_{X}\right)$ um espaço de Banach e $\{S(t)\}_{t \geq 0}$ um $C_{0}$-semigrupo definido o espaço $X$.

Definição 1.6.7. Um semigrupo $S(t): X \rightarrow X, t \geq 0$, é chamado assintoticamente compacto se para qualquer sequência limitada $\left\{x_{n}\right\} \subset X$ e uma sequência numérica $t_{n} \rightarrow \infty$, existe uma subsequência $\left\{S\left(t_{n_{k}}\right)\left(x_{n_{k}}\right)\right\}$ de $\left\{S\left(t_{n}\right)\left(x_{n}\right)\right\}$ convergente em $X$.

Teorema 1.6.3. Seja $S(t): X \rightarrow X, t \geq 0$, um $C_{0}$-semigrupo. Suponhamos que

(i) $S(t)$ possui um conjunto absorvente limitado $\mathcal{B} \subset X$.

(ii) S(t) é assintoticamente compacto.

Então, $S(t)$ possui um atrator global $\mathcal{A} \subset X$, que é dado por

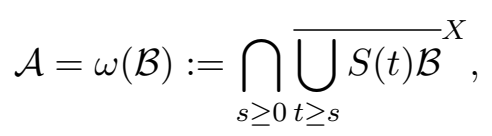

onde $\omega(B)$ é chamado de conjunto $\omega$-limite de um subconjunto $B$ de $X$.

A propriedade de compacidade assintótica requerida pelo Teorema 1.6.3 pode ser verificada por meio de resultados (mais recentes) como veremos a seguir.

Definição 1.6.8. Seja $B$ um subconjunto limitado de $X$. Uma função $\phi(\cdot, \cdot)$ definida sobre $X \times X$ é chamada de função contrativa sobre $B \times B$, se para qualquer sequência $\left\{x_{n}\right\} \subset B$, existe uma subsequência $\left\{x_{n_{k}}\right\}$ de $\left\{x_{n}\right\}$ tal que

$$
\lim _{k \rightarrow \infty} \lim _{l \rightarrow \infty} \phi\left(x_{n_{k}}, x_{n_{l}}\right)=0 .
$$

O conjunto das funções contrativas sobre $B \times B$ será denotado por $\mathcal{C}_{t}(B)$. 
Teorema 1.6.4. Seja $S(t): X \rightarrow X, t \geq 0$, um $C_{0}$-semigrupo que possui um conjunto absorvente limitado $\mathcal{B}$. Se para qualquer $\epsilon \geq 0$ existem um número $T_{0}=T_{0}(\mathcal{B}, \epsilon) \geq 0$ e uma função $\phi_{T}(\cdot, \cdot) \in \mathcal{C}_{t}\left(\mathcal{B}_{0}\right)$ tais que

$$
\|S(T) x-S(T) y\|_{X} \leq \epsilon+\phi_{T}(x, y), \quad \forall x, y \in \mathcal{B},
$$

então $S(t)$ é assintoticamente compacto. Consequentemente, $S(t)$ possui um atrator global $\mathcal{A} \subset X$.

A seguir veremos mais uma alternativa para determinar a existência de atratores globais.

Teorema 1.6.5. Seja $S(t): X \rightarrow X, t \geq 0$, um $C_{0}$-semigrupo que possui um conjunto absorvente limitado $\mathcal{B}$. Suponhamos que $S(t)$ pode ser decomposto como uma soma $S(t)=P(t)+T(t)$, com $P(t)$ e $T(t)$ satisfazendo as seguintes propriedades:

(i) Para cada conjunto limitado $B \subset X$, temos

$$
\sup _{x \in B}\|P(t) x\|_{X} \rightarrow 0 \text { quando } t \rightarrow+\infty .
$$

(ii) Para cada conjunto limitado $B \subset X$, existe $T_{0}=T_{0}(B) \geq 0$ tal que $T(t) B$ é relativamente compacto para todo $t \geq T_{0}$.

Então, $S(t)$ é assintoticamente compacto. Em particular, o conjunto $\mathcal{A}=\omega(\mathcal{B})$ é um atrator global para $S(t)$ em $X$.

\subsubsection{Dimensão fractal}

Buscar uma forma de avaliar a "dimensionalidade" de atratores globais dentro do contexto de sistemas dinâmicos com dimensão infinita constitui uma importante propriedade dos mesmos. Neste trabalho abordaremos uma "medida" comum para atratores globais, sendo conhecida como dimensão fractal. Seguiremos as noções introduzidas por Chueshov \& Lasiecka [25], Hale [55] e Temam [91]. Além disso, algumas ilustrações e aplicações significativas da dimensão fractal podem ser encontradas nos trabalhos de Falconer [37] e Foias \& Olson [42].

Definição 1.6.9. Seja $\mathcal{A}$ um subconjunto compacto de um espaço métrico $X$. A dimensão fractal de $\mathcal{A}$, denotada por $\operatorname{dim}_{f} \mathcal{A}$, é definida por

$$
\operatorname{dim}_{f} \mathcal{A}=\lim _{\varepsilon \rightarrow 0} \sup \frac{\ln \left(n_{\mathcal{A}}(\varepsilon)\right)}{\ln (1 / \varepsilon)}
$$

onde $n_{\mathcal{A}}(\varepsilon)$ é o número mínimo de bolas fechadas de raio $\varepsilon$ que é necessário para cobrir $\mathcal{A}$.

Definição 1.6.10. Uma seminorma $n: X \rightarrow[0, \infty)$ definida sobre um espaço de Banach $X$ é chamada de compacta se para qualquer limitado $B \subset X$, existe uma sequência $\left\{x_{n}\right\} \subset B$ tal que $n\left(x_{m}-x_{n}\right) \rightarrow 0$ quando $m, n \rightarrow \infty$.

Nossa abordagem para estudar dimensão fractal finita de atratores é baseada em um resultado recente devido a Chueshov \& Lasiecka [22, 25], a qual não requer trabalhar com o semigrupo de evolução de classe $C^{1}$ (com respeito a derivada de Fréchet). 
Proposição 1.6.6. Sejam $\left(X,\|\cdot\|_{X}\right)$ um espaço de Banach e $\mathcal{A} \subset X$ um subconjunto fechado e limitado. Suponhamos que existe uma aplicação $\mathcal{T}: \mathcal{A} \rightarrow X$ satisfazendo as seguintes propriedades:

(i) $\mathcal{A} \subseteq \mathcal{T}(\mathcal{A})$

(ii) $\mathcal{T}$ é Lipschitz sobre $\mathcal{A}$, isto é, existe uma constante $L>0$ tal que

$$
\left\|\mathcal{T} y_{1}-\mathcal{T} y_{2}\right\|_{X} \leq L\left\|y_{1}-y_{2}\right\|_{X}, \quad \forall y_{1}, y_{2} \in \mathcal{A} .
$$

(iii) Existem seminormas compactas $n_{1}, n_{2}: X \rightarrow[0, \infty)$ tais que

$$
\left\|\mathcal{T} y_{1}-\mathcal{T} y_{2}\right\|_{X} \leq q\left\|y_{1}-y_{2}\right\|_{X}+K\left[n_{1}\left(y_{1}-y_{2}\right)+n_{2}\left(\mathcal{T} y_{1}-\mathcal{T} y_{2}\right)\right]
$$

para todos $y_{1}, y_{2} \in \mathcal{A}$, onde $0<q<1$ e $K>0$ são constantes.

Então, $\mathcal{A}$ é compacto em $X$ e possui dimensão fractal finita. Além disso, vale a seguinte estimativa

$$
\operatorname{dim}_{f} \mathcal{A} \leq\left[\ln \frac{2}{1+q}\right]^{-1} \ln m_{0}\left(\frac{4 K\left(1+L^{2}\right)^{1 / 2}}{1-q}\right),
$$

onde $m_{0}(R)$ é o número máximo de pares $\left(x_{i}, y_{i}\right) \in X \times X$ com a propriedade

$$
\left\|x_{i}\right\|_{X}^{2}+\left\|y_{i}\right\|_{X}^{2} \leq R^{2}, \quad n_{1}\left(x_{i}-x_{j}\right)+n_{2}\left(y_{i}-y_{j}\right)>1, i \neq j .
$$

Outro resultado interessante envolvendo funções $\alpha$-Hölder contínuas e dimensão fractal é dado pelo Lema 1.2 em Málek \& Pražák [74], o qual expomos a seguir.

Lema 1.6.7. Sejam $X$ e $Y$ espaços métricos e $\mathcal{K} \subset X$ um subconjunto compacto. Se $\mathcal{F}: X \rightarrow Y$ é uma função $\alpha$-Hölder contínua, então

$$
\operatorname{dim}_{f}^{Y} \mathcal{F}(\mathcal{K}) \leq \frac{1}{\alpha} \operatorname{dim}_{f}^{X} \mathcal{K}
$$

Em particular, a dimensão fractal não aumenta sob a ação de uma aplicação Lipschitz contínua. 


$\frac{-10}{2}$

\section{Um modelo de placas com $p$-Laplaciano e memória}

\subsection{Introdução}

Neste capítulo estudamos um problema relativamente mais simples para fixar as ideias de existência e decaimento exponencial de soluções. Entretanto, este caso ilustra uma boa maneira de obter as estimativas envolvendo o operador $p$-Laplaciano e o termo de memória e, por isto, o abordamos em um primeiro momento. Um caso mais geral e com mais detalhes será abordado no Capítulo 3.

Seja $N \geq 1$ natural e considere $\Omega$ um domínio limitado de $\mathbb{R}^{N}$ com fronteira suave $\Gamma=\partial \Omega$ e $\mathbb{R}^{+}=(0, \infty)$. Neste capítulo estudaremos a existência e o comportamento assintótico (decaimento exponencial de energia) de soluções globais para a seguinte equação de placas não linear com memória

$$
\begin{gathered}
u_{t t}+\Delta^{2} u-\Delta_{p} u+\int_{0}^{t} g(t-s) \Delta u(s) d s-\Delta u_{t}+f(u)=0 \quad \text { em } \quad \Omega \times \mathbb{R}^{+}, \\
u=\Delta u=0 \quad \text { sobre } \quad \Gamma \times \mathbb{R}^{+}, \\
u(x, 0)=u_{0}(x) \quad \text { e } \quad u_{t}(x, 0)=u_{1}(x) \quad \text { em } \quad \Omega,
\end{gathered}
$$

onde $\Delta_{p} u$ denota o operador não linear $p$-Laplaciano introduzido na seção 1.3 do Capítulo $1, g$ é usualmente chamada de núcleo da memória e $f(u)$ é uma não linearidade do tipo Lipschitz local. As hipóteses sobre tais termos serão atribuidas na seção seguinte. 
Observação 2.1.1. O termo de memória de segunda ordem que aparece na equação (2.1) representa complicações no decaimento de soluções. Contudo, existe uma indentidade para este termo que vários autores abordaram em seus trabalhos, como por exemplo em [4, 6, 9, 10, 18, 19, 20, 38, 78, 88], entre outros. Na seção seguinte exibiremos tal resultado sobre o termo de memória que, além de facilitar a notação, será bem útil no decorrer deste problema. Já no próximo capítulo abordaremos o problema (2.1)-(2.3) substituindo a memória (sem história para $t<0$ ) por uma memória de quarta ordem (com história para $t<0)$.

Este capítulo está organizado da seguinte maneira. Na seção 2.2 fixaremos as notações preliminares e as hipóteses sobre os devidos termos do problema (2.1)-(2.3). Na seção 2.3 apresentaremos o resultado de existência via método de Faedo-Galerkin, separarando a demonstração em subseções para uma melhor leitura do texto. Por fim, na seção 2.4 determinaremos um resultado de estabilidade exponencial para o sistema (2.1)-(2.3), ou melhor, que a energia associada a este problema possui decaimento exponencial ao longo do tempo.

\subsection{Hipóteses e notações iniciais}

Iniciaremos com as hipóteses precisas sobre o parâmetro $p$ do operador $p$-Laplaciano e sobre as funções $g$ e $f$ presentes em (2.1)-(2.3).

Para $N \in \mathbb{N}$ assumiremos que

$$
2 \leq p \leq \frac{2 N-2}{N-2} \quad \text { se } \quad N \geq 3 \quad \text { e } \quad p \geq 2 \quad \text { se } \quad N=1,2 .
$$

Assumiremos também que o núcleo da memória $g:[0,+\infty) \rightarrow \mathbb{R}^{+}$é uma função limitada de classe $C^{1}$ satisfazendo as propriedades:

$$
g(0)>0 \quad \text { e } \quad l:=1-\mu_{1} \int_{0}^{\infty} g(s) d s>0,
$$

onde $\mu_{1}>0$ é a constante de imersão para $\|\nabla u\|_{2}^{2} \leq \mu_{1}\|\Delta u\|_{2}^{2}$, e existe uma constante $k_{1}>0$ tal que

$$
g^{\prime}(t) \leq-k_{1} g(t), \quad \forall t \geq 0
$$

Consideremos ainda que o termo forçante $f: \mathbb{R} \rightarrow \mathbb{R}$ satisfaz:

$$
f(0)=0, \quad|f(u)-f(v)| \leq k_{2}\left(1+|u|^{\rho}+|v|^{\rho}\right)|u-v|, \quad \forall u, v \in \mathbb{R},
$$

onde $k_{2}>0$ é uma constante e

$$
0<\rho \leq \frac{4}{N-4} \quad \text { se } \quad N \geq 5 \quad \text { e } \quad \rho>0 \quad \text { se } \quad 1 \leq N \leq 4 .
$$

Além disso, suponhamos que

$$
0 \leq \hat{f}(u) \leq f(u) u, \quad \forall u \in \mathbb{R}
$$

onde $\hat{f}(z)=\int_{0}^{z} f(s) d s$. 
Observação 2.2.1. Com a condição (2.4) segue do Teorema de Imersões de Sobolev (ver Teorema 1.2.3 na seção 1.2 do Capítulo 1) que vale a seguinte cadeia de imersões:

$$
H^{2}(\Omega) \cap H_{0}^{1}(\Omega) \hookrightarrow W_{0}^{1,2(p-1)}(\Omega) \hookrightarrow H_{0}^{1}(\Omega) \hookrightarrow L^{2}(\Omega) .
$$

Mais ainda, a condição (2.8) garante que

$$
H^{2}(\Omega) \cap H_{0}^{1}(\Omega) \hookrightarrow L^{2(\rho+1)}(\Omega) .
$$

Já as condições (2.7) e (2.9) incluem funções da forma

$$
f(u) \approx|u|^{\rho} u+|u|^{\alpha} u, \quad 0<\alpha<\rho .
$$

Como já é padrão nesta tese, denotaremos por $(\cdot, \cdot)$ o produto interno em $L^{2}(\Omega)$ e por $\|\cdot\|_{p}$ a norma em $L^{p}(\Omega)$. Além disso, introduzimos os seguintes espaços de Hilbert

$$
\mathcal{H}=\left(H^{2}(\Omega) \cap H_{0}^{1}(\Omega)\right) \times L^{2}(\Omega) \quad \text { e } \quad \mathcal{H}_{1}=H_{\Gamma}^{3}(\Omega) \times H_{0}^{1}(\Omega),
$$

equipados com as normas

$$
\|(u, v)\|_{\mathcal{H}}^{2}=\|\Delta u\|_{2}^{2}+\|v\|_{2}^{2} \quad \text { e } \quad\|(u, v)\|_{\mathcal{H}_{1}}^{2}=\|\nabla \Delta u\|_{2}^{2}+\|\nabla v\|_{2}^{2},
$$

que são provenientes dos respectivos produtos internos em $\mathcal{H}$ e $\mathcal{H}_{1}$, onde

$$
H_{\Gamma}^{3}(\Omega)=\left\{u \in H^{3}(\Omega) \mid u=\Delta u=0 \text { sobre } \Gamma\right\} .
$$

\subsubsection{Uma identidade para a memória}

Agora vamos estabelecer uma identidade relativa ao termo de memória dada pela convolução

$$
(g * u)(t):=\int_{0}^{t} g(t-s) u(s) d s .
$$

Para facilitar a notação em considerações futuras, definamos:

$$
(g \square u)(t)=\int_{0}^{t} g(t-s)\|u(t)-u(s)\|_{2}^{2} d s .
$$

Com isto em mente, temos o

Lema 2.2.1. Sejam $g \in C^{1}\left(\mathbb{R}^{+}\right)$e $u \in C^{1}\left([0, T], H^{2}(\Omega)\right)$. Então, as seguintes identidades se verificam

$$
\begin{aligned}
\int_{0}^{t} g(t-s)\left(\nabla u(s), \nabla u_{t}(t)\right) d s= & -\frac{1}{2} \frac{d}{d t}\left\{(g \square \nabla u)(t)-\left(\int_{0}^{t} g(s) d s\right)\|\nabla u(t)\|_{2}^{2}\right\} \\
& +\frac{1}{2}\left(g^{\prime} \square \nabla u\right)(t)-\frac{1}{2} g(t)\|\nabla u(t)\|_{2}^{2}, \\
\int_{0}^{t} g(t-s)\left(\Delta u(s), \Delta u_{t}(t)\right) d s= & -\frac{1}{2} \frac{d}{d t}\left\{(g \square \Delta u)(t)-\left(\int_{0}^{t} g(s) d s\right)\|\Delta u(t)\|_{2}^{2}\right\} \\
& +\frac{1}{2}\left(g^{\prime} \square \Delta u\right)(t)-\frac{1}{2} g(t)\|\Delta u(t)\|_{2}^{2} .
\end{aligned}
$$

Prova. A prova é obtida diferenciando o termo $g \square y$ para $y=\nabla u$ (e $y=\Delta u$ ), como determinado em Muñoz Rivera et al $[9,10]$. 


\subsection{Existência e unicidade}

Iniciaremos esta seção exibindo o conceito de solução fraca para o problema (2.1)-(2.3) que abordaremos neste capítulo.

Definição 2.3.1. Seja $I=[0, T] \operatorname{com} T>0$. Diremos que uma função $z:=\left(u, u_{t}\right) \in C(I, \mathcal{H})$ é uma solução fraca para o problema (2.1)-(2.3) no intervalo $I$, se $z(0):=\left(u_{0}, u_{1}\right) \in \mathcal{H}$ e

$$
\begin{aligned}
& \frac{d}{d t}\left(u_{t}, \omega\right)+(\Delta u, \Delta \omega)+\left\langle|\nabla u|^{p-2} \nabla u, \nabla \omega\right\rangle \\
& \quad+\int_{0}^{t} g(t-s)(\Delta u(s), \omega) d s+\left(\nabla u_{t}, \nabla \omega\right)+(f(u), \omega)=0
\end{aligned}
$$

quase sempre em $I$, para todo $\omega \in H^{2}(\Omega) \cap H_{0}^{1}(\Omega)$.

Observação 2.3.1. Pelas hipóteses (2.7)-(2.8) é simples verificar que, para $u \in H^{2}(\Omega) \cap H_{0}^{1}(\Omega)$, temos $f(u) \in L^{2}(\Omega)$. Assim, na definição acima faz sentido escrever $(f(u), \omega)$ como um produto interno em $L^{2}(\Omega)$.

Agora estamos aptos a enunciar o principal resultado desta seção, o qual assegura que o problema (2.1)-(2.3) é bem posto de acordo com a Definição 2.3.1.

Teorema 2.3.1. Sob as condições (2.4)-(2.9), temos:

(i) Se $\left(u_{0}, u_{1}\right) \in \mathcal{H}$, então o problema (2.1)-(2.3) possui uma única solução fraca

$$
\left(u, u_{t}\right) \in C([0, T] ; \mathcal{H}), \quad \forall T>0,
$$

satisfazendo

$$
u \in L^{\infty}\left(0, T ; H^{2}(\Omega) \cap H_{0}^{1}(\Omega)\right) \quad e \quad u_{t} \in L^{\infty}\left(0, T ; L^{2}(\Omega)\right) \cap L^{2}\left(0, T ; H_{0}^{1}(\Omega)\right)
$$

(ii) Se $\left(u_{0}, u_{1}\right) \in \mathcal{H}_{1}$, então a solução fraca do problema (2.1)-(2.3) possui maior regularidade

$$
u \in L^{\infty}\left(0, T ; H_{\Gamma}^{3}(\Omega)\right) \quad e \quad u_{t} \in L^{\infty}\left(0, T ; H_{0}^{1}(\Omega)\right) \cap L^{2}\left(0, T ; H^{2}(\Omega) \cap H_{0}^{1}(\Omega)\right)
$$

Prova. A existência de soluções será feita utilizando o método de Faedo-Galerkin. A prova a seguir é baseada em argumentos exibidos por Lions et al [66, 67] e Yang et al [96, 97]. Com respeito ao termo de memória seguiremos ideias análogas às introduzidas por Muñoz Rivera et al $[6,9,10] \mathrm{e}$ por Cavalcanti et al $[18,19]$. Na verdade, o que faremos a seguir é um esboço da demonstração, deixando os detalhes mais intrínsecos deste problema para ser analisado no problema mais geral que será abordado no capítulo seguinte. 


\subsubsection{Problema aproximado}

Denotemos por $\left(\omega_{j}\right)_{j \in \mathbb{N}}$ a base ortonormal bem regular de $L^{2}(\Omega)$, que também é ortogonal em $H_{0}^{1}(\Omega)$ e $H^{2}(\Omega) \cap H_{0}^{1}(\Omega)$, constituida por autofunções do operador biharmônico $\Delta^{2}$ com condições de fronteira $u=\Delta u=0$ sobre $\Gamma$. Para $m \in \mathbb{N}$ consideremos também o subespaço de dimensão finita dado por

$$
V_{m}:=\operatorname{Span}\left\{\omega_{1}, \ldots, \omega_{m}\right\}=\left[\omega_{1}, \ldots, \omega_{m}\right] .
$$

Dado $\left(u_{0}, u_{1}\right) \in \mathcal{H}$, queremos determinar soluções da forma

$$
u^{m}(t)=\sum_{j=1}^{m} y_{m j}(t) \omega_{j},
$$

para o seguinte problema aproximado

$$
\begin{aligned}
& \left(u_{t t}^{m}(t), \omega_{j}\right)+\left(\Delta u^{m}(t), \Delta \omega_{j}\right)+\left\langle\left|\nabla u^{m}(t)\right|^{p-2} \nabla u^{m}(t), \nabla \omega_{j}\right\rangle \\
& \quad+\int_{0}^{t} g(t-s)\left(\Delta u^{m}(s), \omega_{j}\right) d s+\left(\nabla u_{t}^{m}(t), \nabla \omega_{j}\right)+\left(f\left(u^{m}(t)\right), \omega_{j}\right)=0
\end{aligned}
$$

com condições iniciais

$$
u^{m}(0)=u_{0}^{m}, \quad u_{t}^{m}(0)=u_{1}^{m},
$$

onde $u_{0}^{m}$ e $u_{1}^{m}$ são escolhidos de forma que

$$
u_{0}^{m} \rightarrow u_{0} \quad \text { em } \quad H^{2}(\Omega) \cap H_{0}^{1}(\Omega) \quad \text { e } \quad u_{1}^{m} \rightarrow u_{1} \quad \text { em } \quad L^{2}(\Omega) .
$$

Contudo, note que o problema aproximado (2.14)-(2.15) é equivalente a um sistema de equações diferenciais ordinárias, cuja existência de solução local é assegurada pelo Teorema de Carathéodory (ver Coddington \& Levinson [29]). Logo, o problema aproximado (2.14)-(2.15) possui uma solução local $u^{m}(t)$ da forma (2.13) em algum intervalo $\left[0, T_{m}\right)$ com $0<T_{m} \leq T$.

Observação 2.3.2. A passagem acima será feita com mais detalhes no próximo capítulo, onde o problema abordado é mais abrangente do que neste caso.

A seguir apresentaremos estimativas a priori que nos permitirão estender as soluções locais $u^{m}(t)$ ao intervalo $[0, T]$, para qualquer $T>0$ dado, bem como extrair subsequências de soluções convenientemente convergentes para a solução fraca procurada.

\subsubsection{Estimativa a priori 1}

Multiplicando a equação aproximada (2.14) por $u_{t}^{m}(t)$ e integrando sobre $\Omega$, obtemos

$$
\begin{aligned}
\frac{d}{d t}\left\{\frac{1}{2}\left\|u_{t}^{m}(t)\right\|_{2}^{2}+\frac{1}{2}\left\|\Delta u^{m}(t)\right\|_{2}^{2}+\frac{1}{p}\left\|\nabla u^{m}(t)\right\|_{p}^{p}\right. & \left.+\int_{\Omega} \hat{f}\left(u^{m}(t)\right) d x\right\}+\left\|\nabla u_{t}^{m}(t)\right\|_{2}^{2} \\
& =\int_{0}^{t} g(t-s)\left(\nabla u^{m}(s), \nabla u_{t}^{m}(t)\right) d s .
\end{aligned}
$$


Da identidade para memória fornecida pelo Lema 2.2.1 vem que

$$
\begin{aligned}
\int_{0}^{t} g(t-s)\left(\nabla u^{m}(s), \nabla u_{t}^{m}(t)\right) d s= & -\frac{1}{2} \frac{d}{d t}\left\{\left(g \square \nabla u^{m}\right)(t)-\left(\int_{0}^{t} g(s) d s\right)\left\|\nabla u^{m}(t)\right\|_{2}^{2}\right\} \\
& +\frac{1}{2}\left(g^{\prime} \square \nabla u^{m}\right)(t)-\frac{1}{2} g(t)\left\|\nabla u^{m}(t)\right\|_{2}^{2} .
\end{aligned}
$$

Logo, substituindo esta última expressão na anterior, temos

$$
\frac{1}{2} \frac{d}{d t} E^{m}(t)+\left\|\nabla u_{t}^{m}(t)\right\|_{2}^{2}=\frac{1}{2}\left(g^{\prime} \square \nabla u^{m}\right)(t)-\frac{1}{2} g(t)\left\|\nabla u^{m}(t)\right\|_{2}^{2},
$$

onde

$$
\begin{aligned}
E^{m}(t)= & \left\|u_{t}^{m}(t)\right\|_{2}^{2}+\left\|\Delta u^{m}(t)\right\|_{2}^{2}-\left(\int_{0}^{t} g(s) d s\right)\left\|\nabla u^{m}(t)\right\|_{2}^{2}+\frac{2}{p}\left\|\nabla u^{m}(t)\right\|_{p}^{p} \\
& +2 \int_{\Omega} \hat{f}\left(u^{m}(t)\right) d x+\left(g \square \nabla u^{m}\right)(t)
\end{aligned}
$$

Das condições (2.5) e (2.9) e como $\left(g \square \nabla u^{m}\right)(t) \geq 0$, vem que

$$
\left\|u_{t}^{m}(t)\right\|_{2}^{2}+l\left\|\Delta u^{m}(t)\right\|_{2}^{2} \leq E^{m}(t) .
$$

Além disso, da hipótese (2.6),

$$
\left(g^{\prime} \square \nabla u^{m}\right)(t) \leq-k_{1}\left(g \square \nabla u^{m}\right)(t) \leq 0 .
$$

Com isto, o lado direito da igualdade em (2.17) é não positivo. Assim, integrando (2.17) de 0 a $t$ e usando (2.18) chegamos a seguinte estimativa

$$
\left\|u_{t}^{m}(t)\right\|_{2}^{2}+l\left\|\Delta u^{m}(t)\right\|_{2}^{2}+2 \int_{0}^{t}\left\|\nabla u_{t}^{m}(s)\right\|_{2}^{2} d s \leq E^{m}(0) .
$$

Usando agora as hipóteses (2.7) e (2.9) em conjunto com as convergências em (2.16), concluimos

$$
\left\|u_{t}^{m}(t)\right\|_{2}^{2}+\left\|\Delta u^{m}(t)\right\|_{2}^{2}+\int_{0}^{t}\left\|\nabla u_{t}^{m}(s)\right\|_{2}^{2} d s \leq M_{1},
$$

para todos $t \in\left[0, T_{m}\right) \subset[0, T]$ e $m \in \mathbb{N}$, onde $M_{1}=M_{1}\left(\left\|u_{1}\right\|_{2},\left\|\Delta u_{0}\right\|_{2}\right)>0$ é independente de $t$ e $m$. Isto nos permite estender as soluções $u^{m}(t)$ ao intervalo $[0, T] \mathrm{e}$, em particular, notamos que

$$
\begin{aligned}
& \left(u^{m}\right) \text { é limitada em } L^{\infty}\left(0, T ; H^{2}(\Omega) \cap H_{0}^{1}(\Omega)\right), \\
& \left(u_{t}^{m}\right) \text { é limitada em } L^{\infty}\left(0, T ; L^{2}(\Omega)\right) \cap L^{2}\left(0, T ; H_{0}^{1}(\Omega)\right) .
\end{aligned}
$$

\subsubsection{Passagem ao limite e solução fraca}

Das limitações em (2.20)-(2.21) e aplicando os Teoremas 1.2.18 e 1.2.19, existe uma subsequência de $\left(u^{m}\right)$, que ainda denotaremos por $\left(u^{m}\right)$, tal que

$$
\begin{array}{rll}
u^{m} \stackrel{*}{\rightarrow} u & \text { em } & L^{\infty}\left(0, T ; H^{2}(\Omega) \cap H_{0}^{1}(\Omega)\right), \\
u_{t}^{m} \stackrel{*}{\rightarrow} u_{t} & \text { em } & L^{\infty}\left(0, T ; L^{2}(\Omega)\right) \\
u_{t}^{m} \rightarrow u_{t} & \text { em } & L^{2}\left(0, T ; H_{0}^{1}(\Omega)\right) .
\end{array}
$$


Além disso, afirmamos que

$$
u^{m} \rightarrow u \quad \text { em } \quad C\left([0, T], H_{0}^{1}(\Omega)\right) .
$$

De fato, da estimativa (2.19) segue que $\left(u^{m}\right)$ também é limitada no espaço

$$
W:=\left\{u \in L^{2}\left(0, T ; H^{2}(\Omega) \cap H_{0}^{1}(\Omega)\right) ; u_{t} \in L^{2}\left(0, T ; L^{2}(\Omega)\right)\right\},
$$

munido da norma $\|u\|_{W}=\|u\|_{L^{2}\left(0, T ; H^{2}(\Omega) \cap H_{0}^{1}(\Omega)\right)}+\left\|u_{t}\right\|_{L^{2}\left(0, T ; L^{2}(\Omega)\right)}$. Logo, existe uma subsequência de $\left(u^{m}\right)$, que ainda denotaremos por $\left(u^{m}\right)$, tal que

$$
u^{m} \rightarrow u \quad \text { em } W
$$

Como $\left(H^{2}(\Omega) \cap H_{0}^{1}(\Omega)\right) \hookrightarrow \hookrightarrow H_{0}^{1}(\Omega) \hookrightarrow L^{2}(\Omega)$, então pelo Teorema de Aubin-Lions (ver Teorema 1.2.13 ne seção 1.2 do Capítulo 1$)$ segue que $W \hookrightarrow \hookrightarrow L^{2}\left(0, T ; H_{0}^{1}(\Omega)\right)$. Logo,

$$
u^{m} \rightarrow u \quad \text { em } \quad L^{2}\left(0, T ; H_{0}^{1}(\Omega)\right),
$$

de onde segue que

$$
\left\|\nabla u^{m}(t)-\nabla u(t)\right\|_{2} \rightarrow 0 \quad \text { q.s. em } \quad[0, T] .
$$

Agora note que de (2.22) vem que $u^{m}, u, u_{t}^{m}, u_{t} \in L^{2}\left(0, T ; H_{0}^{1}(\Omega)\right)$ e pelo Lema 1.2.14 resulta que $u^{m}, u \in C\left([0, T] ; H_{0}^{1}(\Omega)\right)$. Disto e de (2.25) concluimos que (2.23) vale.

Observação 2.3.3. Uma outra maneira de concluir (2.23) é seguindo a ideia esboçada por Yang $[96,97])$. Com efeito, note que dos limites em $(2.22)$, obtemos

$$
\frac{d}{d t}\left\|\nabla u^{m}(t)-\nabla u(t)\right\|_{2}^{2} \leq 2\left\|\Delta\left(u^{m}(t)-u(t)\right)\right\|_{2}\left\|u_{t}^{m}(t)-u_{t}(t)\right\|_{2} \leq C_{T}, \quad t \in[0, T],
$$

para alguma constante $C_{T}>0$. Isto mostra que

$$
\left\|\nabla u^{m}(\cdot)-\nabla u(\cdot)\right\|_{2}^{2} \in H^{1}[0, T] \hookrightarrow C([0, T]) .
$$

Logo (2.23) segue, uma vez que $\left\|\nabla u^{m}(t)-\nabla u(t)\right\|_{2} \rightarrow 0$ q.s. em $[0, T]$, como em (2.25).

Com estas convergências podemos passar limite no problema aproximado (2.14) e obter a solução fraca para o problema (2.1)-(2.3). De fato, em primeiro lugar consideremos uma função teste $\theta \in \mathcal{D}(0, T)$ e $m, j \in \mathbb{N}$ com $m \geq j$. Multiplicando (2.14) por $\theta$ e integrando sobre $(0, T)$ resulta que

$$
\begin{aligned}
& \int_{0}^{T}\left\{\left(u_{t t}^{m}(t), \omega_{j}\right)+\left(\Delta u^{m}(t), \Delta \omega_{j}\right)+\left\langle\left|\nabla u^{m}(t)\right|^{p-2} \nabla u^{m}(t), \nabla \omega_{j}\right\rangle\right. \\
& \left.\quad+\int_{0}^{t} g(t-s)\left(\Delta u^{m}(s), \omega_{j}\right) d s+\left(\nabla u_{t}^{m}(t), \nabla \omega_{j}\right)+\left(f\left(u^{m}(t)\right), \omega_{j}\right)\right\} \theta(t) d t=0 .
\end{aligned}
$$

Integrando por partes,

$$
\begin{aligned}
-\int_{0}^{T} & \left(u_{t}^{m}(t), \omega_{j}\right) \theta^{\prime}(t) d t+\int_{0}^{T}\left\{\left(\Delta u^{m}(t), \Delta \omega_{j}\right)+\left\langle\left|\nabla u^{m}(t)\right|^{p-2} \nabla u^{m}(t), \nabla \omega_{j}\right\rangle\right. \\
& \left.+\int_{0}^{t} g(t-s)\left(\Delta u^{m}(s), \omega_{j}\right) d s+\left(\nabla u_{t}^{m}(t), \nabla \omega_{j}\right)+\left(f\left(u^{m}(t)\right), \omega_{j}\right)\right\} \theta(t) d t=0 .
\end{aligned}
$$


Neste capítulo daremos atenção somente às convergências dos termos que envolvem o operador $p$-Laplaciano e termo não linear $f$. Os limites dos outros termos em (2.26) segue diretamente de (2.22) e também serão apresentados com mais detalhes no próximo capítulo.

Com respeito ao termo que envole o $p$-Laplaciano, devemos mostrar que

$$
\int_{0}^{T}\left\langle\left|\nabla u^{m}(t)\right|^{p-2} \nabla u^{m}(t), \nabla \omega_{j}\right\rangle \theta(t) d t \stackrel{m \rightarrow \infty}{\longrightarrow} \int_{0}^{T}\left\langle|\nabla u(t)|^{p-2} \nabla u(t), \nabla \omega_{j}\right\rangle \theta(t) d t .
$$

ou seja,

$$
-\int_{0}^{T}\left\langle\Delta_{p}\left(u^{m}(t)\right), \omega_{j}\right\rangle \theta(t) d t \quad \stackrel{m \rightarrow \infty}{\longrightarrow}-\int_{0}^{T}\left\langle\Delta_{p}(u(t)), \omega_{j}\right\rangle \theta(t) d t
$$

De fato, em primeiro lugar notamos que pelo Lema 1.3.1, existe uma constante $M=M(p, N)>0$ tal que

$$
\left.|| x\right|^{p-2} x-|y|^{p-2} y\left|\leq M\left(|x|^{p-2}+|y|^{p-2}\right)\right| x-y \mid, \quad \forall x, y \in \mathbb{R}^{N} .
$$

Em particular, para $x=\nabla u^{m}$ e $y=\nabla u$, segue que

$$
\left.|| \nabla u^{m}\right|^{p-2} \nabla u^{m}-|\nabla u|^{p-2} \nabla u\left|\leq M\left(\left|\nabla u^{m}\right|^{p-2}+|\nabla u|^{p-2}\right)\right| \nabla u^{m}-\nabla u \mid .
$$

Disto e usando a Desigualdade generalizada de Hölder com $\frac{p-2}{2(p-1)}+\frac{1}{2(p-1)}+\frac{1}{2}=1$, a hipótese (2.4), a estimativa (2.19) e a convergência (2.22), então

$$
\begin{aligned}
\mid \int_{0}^{T} & \left\langle-\Delta_{p}\left(u^{m}(t)\right)+\Delta_{p}(u(t)), \omega_{j}\right\rangle \theta(t) d t \mid \\
& =\left|\int_{0}^{T}\left\langle\left|\nabla u^{m}(t)\right|^{p-2} \nabla u^{m}(t)-|\nabla u(t)|^{p-2} \nabla u(t), \nabla \omega_{j}\right\rangle \theta(t) d t\right| \\
& \leq \int_{0}^{T}\left|\left\langle\left|\nabla u^{m}(t)\right|^{p-2} \nabla u^{m}(t)-|\nabla u(t)|^{p-2} \nabla u(t), \nabla \omega_{j}\right\rangle\right||\theta(t)| d t \\
& \leq\left.\|\theta\|_{\infty} \int_{0}^{T} \int_{\Omega}|| \nabla u^{m}(t)\right|^{p-2} \nabla u^{m}(t)-|\nabla u(t)|^{p-2} \nabla u(t)|| \nabla \omega_{j} \mid d x d t \\
& \leq C_{p} \int_{0}^{T} \int_{\Omega}\left(\left|\nabla u^{m}(t)\right|^{p-2}+|\nabla u(t)|^{p-2}\right)\left|\nabla u^{m}(t)-\nabla u(t) \| \nabla \omega_{j}\right| d x d t \\
& \leq C_{p} \int_{0}^{T}\left(\left\|\nabla u^{m}(t)\right\|_{2(p-1)}^{p-2}+\|\nabla u(t)\|_{2(p-1)}^{p-2}\right)\left\|\nabla u^{m}(t)-\nabla u(t)\right\|_{2}\left\|\nabla \omega_{j}\right\|_{2(p-1)} d t \\
& \leq C_{p} \int_{0}^{T}\left(\left\|\Delta u^{m}(t)\right\|_{2}^{p-2}+\|\Delta u(t)\|_{2}^{p-2}\right)\left\|\nabla u^{m}(t)-\nabla u(t)\right\|_{2}\left\|\Delta \omega_{j}\right\|_{2} d t \\
& \leq C_{p} \int_{0}^{T}\left\|\nabla u^{m}(t)-\nabla u(t)\right\|_{2} d t,
\end{aligned}
$$

para alguma constante $C_{p}>0$, isto é, existe uma constante $C>0$ tal que

$$
\left|\int_{0}^{T}\left\langle-\Delta_{p}\left(u^{m}(t)\right)+\Delta_{p}(u(t)), \omega_{j}\right\rangle \theta(t) d t\right| \leq C \int_{0}^{T}\left\|\nabla u^{m}(t)-\nabla u(t)\right\|_{2} d t .
$$

Agora utilizando a convergência (2.23) em (2.28), então o limite (2.27) segue. 
Por outro lado, com respeito ao termo não linear $f$, devemos mostrar

$$
\int_{0}^{T}\left(f\left(u^{m}(t)\right), \omega_{j}\right) \theta(t) d t \quad \stackrel{m \rightarrow \infty}{\longrightarrow} \int_{0}^{T}\left(f(u(t)), \omega_{j}\right) \theta(t) d t .
$$

Primeiramente, usando a desigualdade de Hölder generalizada com $\frac{\rho}{2(\rho+1)}+\frac{1}{2(\rho+1)}+\frac{1}{2}=1$, as condições (2.7)-(2.8), a estimativa (2.19) e a convergência (2.22), então para qualquer $\phi \in$ $H^{2}(\Omega) \cap H_{0}^{1}(\Omega) \hookrightarrow L^{2(\rho+1)}(\Omega)$, obtemos

$$
\begin{aligned}
& \left|\left(f\left(u^{m}(t)\right)-f(u(t)), \phi\right)\right| \\
& \quad \leq \int_{\Omega}\left|f\left(u^{m}(t)\right)-f(u(t))\right||\phi| d x \\
& \quad \leq k_{2} \int_{\Omega}\left(1+\left|u^{m}(t)\right|^{\rho}+|u(t)|^{\rho}\right)\left|u^{m}(t)-u(t) \| \phi\right| d x \\
& \quad \leq k_{2}\left(|\Omega|^{\frac{\rho}{2(\rho+1)}}+\left\|u^{m}(t)\right\|_{2(\rho+1)}^{\rho}+\|u(t)\|_{2(\rho+1)}^{\rho}\right)\left\|u^{m}(t)-u(t)\right\|_{2}\|\phi\|_{2(\rho+1)} \\
& \quad \leq C_{\rho}\left(|\Omega|^{\frac{\rho}{2(\rho+1)}}+\left\|\Delta u^{m}(t)\right\|_{2}^{\rho}+\|\Delta u(t)\|_{2}^{\rho}\right)\left\|u^{m}(t)-u(t)\right\|_{2}\|\Delta \phi\|_{2} \\
& \quad \leq C_{\rho}\left\|u^{m}(t)-u(t)\right\|_{2}\|\Delta \phi\|_{2},
\end{aligned}
$$

para alguma constante $C_{\rho}>0$. Assim, existe uma constante $C>0$ tal que

$$
\left\|f\left(u^{m}(t)\right)-f(u(t))\right\|_{\left[H^{2}(\Omega) \cap H_{0}^{1}(\Omega)\right]^{\prime}} \leq C\left\|u^{m}(t)-u(t)\right\|_{2} .
$$

Disto e aplicando a convergência (2.23) vem que

$$
\begin{aligned}
\mid \int_{0}^{T}\left(f\left(u^{m}(t)\right)-\right. & \left.f(u(t)), \omega_{j}\right) \theta(t) d t \mid \\
& \leq \int_{0}^{T}\left|\left(f\left(u^{m}(t)\right)-f(u(t)), \omega_{j}\right)\right||\theta(t)| d t \\
& \leq C \int_{0}^{T}\left\|f\left(u^{m}(t)\right)-f(u(t))\right\|_{\left[H^{2}(\Omega) \cap H_{0}^{1}(\Omega)\right]^{\prime}}\left\|\Delta \omega_{j}\right\|_{2} d t \\
& \leq C \int_{0}^{T}\left\|u^{m}(t)-u(t)\right\|_{2} d t \\
& \leq C \int_{0}^{T}\left\|\nabla u^{m}(t)-\nabla u(t)\right\|_{2} d t \stackrel{m \rightarrow \infty}{\longrightarrow} 0 .
\end{aligned}
$$

Isto prova o limite (2.29). Como já comentamos, os outros termos em (2.26) convergem de modo padrão usando os limites obtidos em (2.22). Assim sendo, passando o limite quando $m \rightarrow \infty$ em (2.26) resulta que

$$
\begin{aligned}
-\int_{0}^{T}\left(u_{t}(t), \omega_{j}\right) \theta^{\prime}(t) d t+\int_{0}^{T}\left\{\left(\Delta u(t), \Delta \omega_{j}\right)+\left\langle|\nabla u(t)|^{p-2} \nabla u(t), \nabla \omega_{j}\right\rangle\right. \\
\left.\quad+\int_{0}^{t} g(t-s)\left(\Delta u(s), \omega_{j}\right) d s+\left(\nabla u_{t}(t), \nabla \omega_{j}\right)+\left(f(u(t)), \omega_{j}\right)\right\} \theta(t) d t=0,
\end{aligned}
$$

ou seja,

$$
\begin{aligned}
\int_{0}^{T} & \frac{d}{d t}\left(u_{t}(t), \omega_{j}\right) \theta(t) d t+\int_{0}^{T}\left\{\left(\Delta u(t), \Delta \omega_{j}\right)+\left\langle|\nabla u(t)|^{p-2} \nabla u(t), \nabla \omega_{j}\right\rangle\right. \\
& \left.+\int_{0}^{t} g(t-s)\left(\Delta u(s), \omega_{j}\right) d s+\left(\nabla u_{t}(t), \nabla \omega_{j}\right)+\left(f(u(t)), \omega_{j}\right)\right\} \theta(t) d t=0,
\end{aligned}
$$


para todo $j \in \mathbb{N}$ e $\theta \in \mathcal{D}(0, T)$.

Como $\left(\omega_{j}\right)_{j \in \mathbb{N}}$ constitui uma base para $H^{2}(\Omega) \cap H_{0}^{1}(\Omega)$, então

$$
\begin{aligned}
\int_{0}^{T}\left\{\frac{d}{d t}\right. & \left(u_{t}(t), \omega\right)+(\Delta u(t), \Delta \omega)-\left\langle\Delta_{p} u(t), \omega\right\rangle \\
& \left.+\int_{0}^{t} g(t-s)(\Delta u(s), \omega) d s+\left(\nabla u_{t}(t), \nabla \omega\right)+(f(u(t)), \omega)\right\} \theta(t) d t=0
\end{aligned}
$$

para todo $\omega \in H^{2}(\Omega) \cap H_{0}^{1}(\Omega)$ e $\theta \in \mathcal{D}(0, T)$. Logo, de (2.30) inferimos que

$$
\begin{aligned}
\frac{d}{d t}\left(u_{t}, \omega\right)+(\Delta u, \Delta \omega)-\left\langle\Delta_{p} u, \omega\right\rangle & +\int_{0}^{t} g(t-s)(\Delta u(s), \omega) d s \\
& +\left(\nabla u_{t}, \nabla \omega\right)+(f(u), \omega)=0 \quad \text { em } \quad \mathcal{D}^{\prime}(0, T),
\end{aligned}
$$

para todo $\omega \in H^{2}(\Omega) \cap H_{0}^{1}(\Omega)$. Isto mostra a função $u$ satisfaz (2.10) com

$$
\begin{aligned}
& u \in L^{\infty}\left(0, T ; H^{2}(\Omega) \cap H_{0}^{1}(\Omega)\right), \\
& u_{t} \in L^{\infty}\left(0, T ; L^{2}(\Omega)\right) \cap L^{2}\left(0, T ; H_{0}^{1}(\Omega)\right),
\end{aligned}
$$

de onde segue também a condição (2.11). Resta verificar que $\left(u, u_{t}\right) \in C([0, T], \mathcal{H})$ e que valem as condições iniciais $u(0)=u_{0}$ e $u_{t}(0)=u_{1}$. Contudo, a continuidade de $u$ e $u_{t}$ em $[0, T]$ segue de modo análogo a Yang \& Jin [97] e será feita com mais detalhes no próximo capítulo. As condições iniciais também serão verificadas posteriormente. Isto conclui que a função $z=\left(u, u_{t}\right)$ é uma solução fraca para o problema (2.1)-(2.3)

Por outro lado, identificando $L^{2}:=L^{2}(\Omega)$ com seu dual, por meio do Teorema de Representação de Riesz-Fréchet, temos a seguinte cadeia de inclusões contínuas

$$
H^{2} \cap H_{0}^{1} \hookrightarrow W_{0}^{1, p} \hookrightarrow H_{0}^{1} \hookrightarrow L^{2} \hookrightarrow H^{-1} \hookrightarrow W^{-1, p^{\prime}} \hookrightarrow\left[H^{2} \cap H_{0}^{1}\right]^{\prime}
$$

Com isto podemos reescrever a expressão em (2.30), depois de integrar por partes, como

$$
\begin{aligned}
& -\left\langle\int_{0}^{T} u_{t}(t) \theta^{\prime}(t) d t, \omega\right\rangle+\left\langle\int_{0}^{T} \Delta^{2} u(t) \theta(t) d t, \omega\right\rangle-\left\langle\int_{0}^{T} \Delta_{p} u(t) \theta(t) d t, \omega\right\rangle \\
& +\left\langle\int_{0}^{T}(g * \Delta u)(t) \theta(t) d t, \omega\right\rangle-\left\langle\int_{0}^{T} \Delta u_{t}(t) \theta(t) d t, \omega\right\rangle+\left\langle\int_{0}^{T} f(u(t)) \theta(t) d t, \omega\right\rangle=0,
\end{aligned}
$$

onde relembramos que $(g * \Delta u)(t)=\int_{0}^{t} g(t-s) \Delta u(s) d s$. Novamente usando integração por partes,

$$
\begin{aligned}
& \left\langle\int_{0}^{T} u_{t t}(t) \theta(t) d t, \omega\right\rangle+\left\langle\int_{0}^{T} \Delta^{2} u(t) \theta(t) d t, \omega\right\rangle-\left\langle\int_{0}^{T} \Delta_{p} u(t) \theta(t) d t, \omega\right\rangle \\
& +\left\langle\int_{0}^{T}(g * \Delta u)(t) \theta(t) d t, \omega\right\rangle-\left\langle\int_{0}^{T} \Delta u_{t}(t) \theta(t) d t, \omega\right\rangle+\left\langle\int_{0}^{T} f(u(t)) \theta(t) d t, \omega\right\rangle=0,
\end{aligned}
$$

para todo $\omega \in H^{2}(\Omega) \cap H_{0}^{1}(\Omega)$, onde nesta última igualdade a notação

$$
\langle\cdot, \cdot\rangle \text { significa a dualidade }\langle\cdot, \cdot\rangle_{\left[H^{2}(\Omega) \cap H_{0}^{1}(\Omega)\right]^{\prime}, H^{2}(\Omega) \cap H_{0}^{1}(\Omega)} \cdot
$$


Portanto, concluimos que

$$
u_{t t}+\Delta^{2} u-\Delta_{p} u+(g * \Delta u)-\Delta u_{t}+f(u)=0 \quad \text { em } \quad \mathcal{D}^{\prime}\left(0, T ;\left[H^{2}(\Omega) \cap H_{0}^{1}(\Omega)\right]^{\prime}\right) .
$$

Mais ainda, usando (2.31)-(2.32) e as inclusões em (2.33) deduzimos facilmente que

$$
\begin{aligned}
& \Delta^{2} u \in L^{\infty}\left(0, T ;\left[H^{2}(\Omega) \cap H_{0}^{1}(\Omega)\right]^{\prime}\right), \\
& \Delta u_{t} \in L^{2}\left(0, T ;\left[H_{0}^{1}(\Omega)\right]^{\prime}\right) \hookrightarrow L^{2}\left(0, T ;\left[H^{2}(\Omega) \cap H_{0}^{1}(\Omega)\right]^{\prime}\right), \\
& (g * \Delta u), f(u) \in L^{\infty}\left(0, T ; L^{2}(\Omega)\right) \hookrightarrow L^{\infty}\left(0, T ;\left[H^{2}(\Omega) \cap H_{0}^{1}(\Omega)\right]^{\prime}\right) .
\end{aligned}
$$

Resta verificar que

$$
\Delta_{p} u \in L^{\infty}\left(0, T ;\left[H^{2}(\Omega) \cap H_{0}^{1}(\Omega)\right]^{\prime}\right) .
$$

De fato, para $\phi \in H^{2}(\Omega) \cap H_{0}^{1}(\Omega) \hookrightarrow W_{0}^{1,2(p-1)}(\Omega)$, temos

$$
\begin{aligned}
\left|\left\langle\Delta_{p} u(t), \phi\right\rangle\right| & =\left|\left\langle|\nabla u(t)|^{p-2} \nabla u(t), \nabla \phi\right\rangle\right| \\
& \leq \int_{\Omega}|\nabla u(t)|^{p-1}|\nabla \phi| d x \\
& \leq\left[\int_{\Omega}|\nabla u(t)|^{2(p-1)} d x\right]^{1 / 2}\left[\int_{\Omega}|\nabla \phi|^{2} d x\right]^{1 / 2} \\
& =\|\nabla u(t)\|_{2(p-1)}^{p-1}\|\nabla \phi\|_{2} \\
& \leq C_{p}\|\Delta u(t)\|_{2}^{p-1}\|\Delta \phi\|_{2},
\end{aligned}
$$

para alguma constante $C_{p}>0$. Assim, de (2.31) existe uma constante $C>0$ tal que

$$
\left\|\Delta_{p} u(t)\right\|_{\left[H^{2}(\Omega) \cap H_{0}^{1}(\Omega)\right]^{\prime}} \leq C \quad \text { q.s. em } \quad[0, T] .
$$

Finalmente, combinando (2.34) com (2.35)-(2.36) concluimos

$$
u_{t t}+\Delta^{2} u-\Delta_{p} u+(g * \Delta u)-\Delta u_{t}+f(u)=0 \quad \text { in } \quad L^{2}\left(0, T ;\left[H^{2}(\Omega) \cap H_{0}^{1}(\Omega)\right]^{\prime}\right) .
$$

Isto encerra a prova do item $(i)$ do Teorema 2.3.1.

\subsubsection{Estimativa a priori 2}

Agora consideramos $\left(u_{0}, u_{1}\right) \in \mathcal{H}_{1}$ e seja $u^{m}(t)$ da forma (2.13) uma solução do problema aproximado (2.14)-(2.15), onde agora

$$
u_{0}^{m} \rightarrow u_{0} \quad \text { em } \quad H_{\Gamma}^{3}(\Omega) \quad \text { e } \quad u_{1}^{m} \rightarrow u_{1} \quad \text { em } \quad H_{0}^{1}(\Omega)
$$

Pela escolha da base $\left(\omega_{j}\right)$, podemos multiplicar o problema aproximado (2.14) por $-\Delta u_{t}^{m} \mathrm{e}$ integrando por partes, obtemos

$$
\begin{aligned}
\frac{1}{2} \frac{d}{d t}\left\{\left\|\nabla u_{t}^{m}(t)\right\|_{2}^{2}\right. & \left.+\left\|\nabla \Delta u^{m}(t)\right\|_{2}^{2}\right\}+\left\langle\Delta_{p} u^{m}(t), \Delta u_{t}^{m}(t)\right\rangle+\left\|\Delta u_{t}^{m}(t)\right\|_{2}^{2} \\
& =\left(f\left(u^{m}(t)\right), \Delta u_{t}^{m}(t)\right)+\int_{0}^{t} g(t-s)\left(\Delta u^{m}(s), \Delta u_{t}^{m}(t)\right) d s .
\end{aligned}
$$


Notando que

$$
\left\langle\Delta_{p} u^{m}, \Delta u_{t}^{m}\right\rangle=\frac{d}{d t}\left\langle\Delta_{p} u^{m}, \Delta u^{m}\right\rangle-J_{1},
$$

onde

$$
J_{1}=-\int_{\Omega}\left\{(p-2)\left|\nabla u^{m}\right|^{p-4}\left(\nabla u^{m} \cdot \nabla u_{t}^{m}\right) \nabla u^{m}+\left|\nabla u^{m}\right|^{p-2} \nabla u_{t}^{m}\right\} \cdot \nabla \Delta u^{m} d x,
$$

derivamos a seguinte igualdade

$$
\begin{aligned}
\frac{1}{2} \frac{d}{d t}\left\{\left\|\nabla u_{t}^{m}(t)\right\|_{2}^{2}+\left\|\nabla \Delta u^{m}(t)\right\|_{2}^{2}\right. & \left.+2\left\langle\Delta_{p} u^{m}(t), \Delta u^{m}(t)\right\rangle\right\} \\
& +\left\|\Delta u_{t}^{m}(t)\right\|_{2}^{2}=J_{1}+J_{2}+J_{3},
\end{aligned}
$$

onde

$$
\begin{aligned}
& J_{2}=\left(f\left(u^{m}(t)\right), \Delta u_{t}^{m}(t)\right)=\int_{\Omega} f\left(u^{m}(t)\right) \Delta u_{t}^{m}(t) d x, \\
& J_{3}=\int_{0}^{t} g(t-s)\left(\Delta u^{m}(s), \Delta u_{t}^{m}(t)\right) d s .
\end{aligned}
$$

No que segue vamos estimar o lado direito de (2.38). Para facilitar a notação o mesmo símbolo $C$ denotará diferentes constantes positivas que aparecerão no texto.

Da estimativa (2.19) e usando a desigualdade de Hölder generalizada com $\frac{p-2}{2(p-1)}+\frac{1}{2(p-1)}+\frac{1}{2}=1$, temos

$$
\begin{aligned}
\left|J_{1}\right| & \leq(p-1) \int_{\Omega}\left|\nabla u^{m}(t)\right|^{p-2}\left|\nabla u_{t}^{m}(t)\right|\left|\nabla \Delta u^{m}(t)\right| d x \\
& \leq(p-1)\left\|\nabla u^{m}(t)\right\|_{2(p-1)}^{p-2}\left\|\nabla u_{t}^{m}(t)\right\|_{2(p-1)}\left\|\nabla \Delta u^{m}(t)\right\|_{2} \\
& \leq C_{p}\left\|\nabla u_{t}^{m}(t)\right\|_{2(p-1)}\left\|\nabla \Delta u^{m}(t)\right\|_{2},
\end{aligned}
$$

para alguma constante $C_{p}>0$. Como $H^{2}(\Omega) \cap H_{0}^{1}(\Omega) \hookrightarrow W_{0}^{1,2(p-1)}(\Omega)$ e $u_{t}^{m}$ é bem regular,

$$
\left\|\nabla u_{t}^{m}(t)\right\|_{2(p-1)}^{2} \leq \mu_{2}\left\|\Delta u_{t}^{m}(t)\right\|_{2}^{2}
$$

onde $\mu_{2}>0$ é a constante de imersão correspondente. Usando a desigualdade de Young, existe uma constante $C>0$ tal que

$$
\left|J_{1}\right| \leq \frac{1}{4}\left\|\Delta u_{t}^{m}(t)\right\|_{2}^{2}+\frac{C}{2}\left\|\nabla \Delta u^{m}(t)\right\|_{2}^{2} .
$$

Além disso, das hipóteses (2.7)-(2.8), da estimativa (2.19) e como $H_{\Gamma}^{3}(\Omega) \hookrightarrow H^{2}(\Omega) \cap H_{0}^{1}(\Omega) \hookrightarrow$ $L^{2(\rho+1)}(\Omega)$, segue que

$$
\begin{aligned}
\left\|f\left(u^{m}(t)\right)\right\|_{2}^{2} & =\int_{\Omega}\left|f\left(u^{m}(t)\right)\right|^{2} d x \\
& \leq 2 k_{2} \int_{\Omega}\left(\left|u^{m}(t)\right|^{2}+\left|u^{m}(t)\right|^{2(\rho+1)}\right) d x \\
& =2 k_{2}\left(\left\|u^{m}(t)\right\|_{2}^{2}+\left\|u^{m}(t)\right\|_{2(\rho+1)}^{2(\rho+1)}\right) \\
& \leq C_{\rho}\left(\left\|\Delta u^{m}(t)\right\|_{2}^{2}+\left\|\Delta u^{m}(t)\right\|_{2}^{2(\rho+1)}\right) \\
& =C_{\rho}\left(1+\left\|\Delta u^{m}(t)\right\|_{2}^{2 \rho}\right)\left\|\Delta u^{m}(t)\right\|_{2}^{2} \\
& \leq \frac{C}{2}\left\|\nabla \Delta u^{m}(t)\right\|_{2}^{2}
\end{aligned}
$$


para alguma constante $C>0$. Assim, novamente aplicando a desigualdade de Young,

$$
\begin{aligned}
\left|J_{2}\right| & \leq\left\|f\left(u^{m}(t)\right)\right\|_{2}\left\|\Delta u_{t}^{m}(t)\right\|_{2} \\
& \leq\left\|f\left(u^{m}(t)\right)\right\|_{2}^{2}+\frac{1}{4}\left\|\Delta u_{t}^{m}(t)\right\|_{2}^{2} \\
& \leq \frac{C}{2}\left\|\nabla \Delta u^{m}(t)\right\|_{2}^{2}+\frac{1}{4}\left\|\Delta u_{t}^{m}(t)\right\|_{2}^{2} .
\end{aligned}
$$

Usando mais uma vez a estimativa (2.19) e a desigualdade de Young,

$$
\begin{aligned}
\left|J_{3}\right| & \leq\left(\int_{0}^{t} g(t-s)\left\|\Delta u^{m}(s)\right\|_{2} d s\right)\left\|\Delta u_{t}^{m}(t)\right\|_{2} \\
& \leq M_{1}\|g\|_{L^{1}\left(\mathbb{R}^{+}\right)}\left\|\Delta u_{t}^{m}(t)\right\|_{2} \\
& \leq C+\frac{1}{4}\left\|\Delta u_{t}^{m}(t)\right\|_{2}^{2} .
\end{aligned}
$$

Substituindo (2.39)-(2.41) em (2.38) e pondo

$$
F^{m}(t)=\left\|\nabla u_{t}^{m}(t)\right\|_{2}^{2}+\left\|\nabla \Delta u^{m}(t)\right\|_{2}^{2}+2\left\langle\Delta_{p} u^{m}(t), \Delta u^{m}(t)\right\rangle+2 C,
$$

então

$$
\frac{d}{d t} F^{m}(t)+\frac{1}{2}\left\|\Delta u_{t}^{m}(t)\right\|_{2}^{2} \leq 2 C+2 C\left\|\nabla \Delta u^{m}(t)\right\|_{2}^{2} .
$$

Agora notamos que

$$
\begin{aligned}
\left|\left\langle\Delta_{p} u^{m}(t), \Delta u^{m}(t)\right\rangle\right| & =\left|\left\langle\left|\nabla u^{m}(t)\right|^{p-2} \nabla u^{m}(t), \nabla \Delta u^{m}(t)\right\rangle\right| \\
& \leq \int_{\Omega}\left|\nabla u^{m}(t)\right|^{p-1}\left|\nabla \Delta u^{m}(t)\right| d x \\
& \leq\left\|\nabla u^{m}(t)\right\|_{2(p-1)}^{p-1}\left\|\nabla \Delta u^{m}(t)\right\|_{2} \\
& \leq\left\|\nabla u^{m}(t)\right\|_{2(p-1)}^{2(p-1)}+\frac{1}{4}\left\|\nabla \Delta u^{m}(t)\right\|_{2}^{2} \\
& \leq C_{p}\left\|\Delta u^{m}(t)\right\|_{2}^{2(p-1)}+\frac{1}{4}\left\|\nabla \Delta u^{m}(t)\right\|_{2}^{2} .
\end{aligned}
$$

Pela estimativa (2.19), existe uma constante $C>0$ tal que

$$
\left|\left\langle\Delta_{p} u^{m}(t), \Delta u^{m}(t)\right\rangle\right| \leq C+\frac{1}{4}\left\|\nabla \Delta u^{m}(t)\right\|_{2}^{2},
$$

de onde segue que

$$
\frac{1}{2}\|\nabla \Delta u(t)\|_{2}^{2}+2 C+2\left\langle\Delta_{p} u^{m}(t), \Delta u^{m}(t)\right\rangle \geq 0 .
$$

Combinando (2.42) e (2.43), existe uma constante $C>0$ tal que

$$
\frac{d}{d t} F^{m}(t)+\frac{1}{2}\left\|\Delta u_{t}^{m}(t)\right\|_{2}^{2} \leq C F^{m}(t) .
$$

Agora, integrando (2.44) sobre $(0, t) \subset[0, T]$ e explorando os limites (2.37) resulta

$$
F^{m}(t)+\frac{1}{2} \int_{0}^{t}\left\|\Delta u_{t}^{m}(\tau)\right\|_{2}^{2} d \tau \leq C+C \int_{0}^{t} F^{m}(\tau) d \tau
$$


Portanto, usando a desigualdade de Growall e novamente (2.43) concluimos

$$
\left\|\nabla u_{t}^{m}(t)\right\|_{2}^{2}+\left\|\nabla \Delta u^{m}(t)\right\|_{2}^{2}+\int_{0}^{t}\left\|\Delta u_{t}^{m}(\tau)\right\|_{2}^{2} d \tau \leq M_{2}
$$

para todo $t \in[0, T]$ e $m \in \mathbb{N}$, onde $M_{2}=M_{2}\left(\left\|\nabla u_{1}\right\|_{2},\left\|\nabla \Delta u_{0}\right\|_{2}, T\right)>0$.

Em particular,

$$
\begin{aligned}
& \left(u^{m}\right) \text { é limitada em } L^{\infty}\left(0, T ; H_{\Gamma}^{3}(\Omega)\right), \\
& \left(u_{t}^{m}\right) \text { é limitada em } L^{\infty}\left(0, T ; H_{0}^{1}(\Omega)\right) \cap L^{2}\left(0, T ; H^{2}(\Omega) \cap H_{0}^{1}(\Omega)\right) .
\end{aligned}
$$

\subsubsection{Passagem ao limite e solução fraca mais regular}

As limitações em (2.46)-(2.47) são suficientes para passar limite no problema aproximado e garantir que, quando os dados iniciais $\left(u_{0}, u_{1}\right) \in \mathcal{H}_{1}$, a solução fraca de (2.1)-(2.3) possuem mais regularidade

$$
\begin{aligned}
& u \in L^{\infty}\left(0, T ; H_{\Gamma}^{3}(\Omega)\right), \\
& u_{t} \in L^{\infty}\left(0, T ; H_{0}^{1}(\Omega)\right) \cap L^{2}\left(0, T ; H^{2}(\Omega) \cap H_{0}^{1}(\Omega)\right) .
\end{aligned}
$$

A conclusão do item (ii) do Teorema 2.3.1 segue, então, de maneira similar ao caso fraco. No próximo capítulo esboçaremos mais detalhes que serão suficientes para cobrir este caso.

Além disso, como também veremos mais adiante, podemos concluir que neste caso $u_{t t} \in$ $L^{2}\left(0, T ; H^{-1}(\Omega)\right)$, ou seja, que $u$ é satisfaz a equação

$$
u_{t t}+\Delta^{2} u-\Delta_{p} u+(g * \Delta u)-\Delta u_{t}+f(u)=0 \quad \text { em } \quad L^{2}\left(0, T ; H^{-1}(\Omega)\right) .
$$

\subsubsection{Unicidade}

Sejam $u$ e $v$ duas soluções mais regulares do problema (2.1)-(2.3) e considere $w=u-v$. Então $w$ satisfaz a equação

$$
w_{t t}+\Delta^{2} w-\Delta w_{t}=\Delta_{p} u-\Delta_{p} v-f(u)+f(v)-\int_{0}^{t} g(t-s) \Delta w(s) d s
$$

em $L^{2}\left(0, T ; H^{-1}(\Omega)\right)$, com condição de fronteira $w=\Delta w=0$ sobre $\Gamma$ e dados iniciais nulos. Como $w_{t} \in L^{2}\left(0, T ; H_{0}^{1}(\Omega)\right)$, podemos multiplicar a equação acima por $w_{t}(t)$ e integrando por partes, obtemos

$$
\begin{aligned}
\frac{d}{d t}\left\{\frac{1}{2}\left\|w_{t}(t)\right\|_{2}^{2}\right. & \left.+\frac{1}{2}\|\Delta w(t)\|_{2}^{2}\right\}+\left\|\nabla w_{t}(t)\right\|_{2}^{2} \\
= & \left\langle\Delta_{p} u(t)-\Delta_{p} v(t), w_{t}(t)\right\rangle-\left(f(u(t))-f(v(t)), w_{t}(t)\right) \\
& +\int_{0}^{t} g(t-s)\left(\nabla w(s), \nabla w_{t}(t)\right) d s
\end{aligned}
$$


Usando o Lema 2.2.1, deduzimos que

$$
\begin{gathered}
\frac{1}{2} \frac{d}{d t}\left\{\left\|w_{t}(t)\right\|_{2}^{2}+\|\Delta w(t)\|_{2}^{2}-\left(\int_{0}^{t} g(s) d s\right)\|\nabla w(t)\|_{2}^{2}+(g \square \nabla w)(t)\right\}+\left\|\nabla w_{t}(t)\right\|_{2}^{2} \\
\leq\left|\left\langle\Delta_{p} u(t)-\Delta_{p} v(t), w_{t}(t)\right\rangle\right|+\int_{\Omega}|f(u(t))-f(v(t))|\left|w_{t}(t)\right| d x
\end{gathered}
$$

Como anteriormente segue do Lema 1.3.1 que

$$
\begin{aligned}
\left|\left\langle\Delta_{p} u(t)-\Delta_{p} v(t), w_{t}(t)\right\rangle\right| & \leq C\left(\|\nabla u(t)\|_{2(p-1)}^{p-2}+\|\nabla v(t)\|_{2(p-1)}^{p-2}\right)\|\nabla w(t)\|_{2(p-1)}\left\|\nabla w_{t}(t)\right\|_{2} \\
& \leq C\|\Delta w(t)\|_{2}^{2}+\frac{1}{2}\left\|\nabla w_{t}(t)\right\|_{2}^{2}
\end{aligned}
$$

para alguma constante $C>0$.

Além disso, das condições (2.7)-(2.8) e como $\frac{\rho}{2(\rho+1)}+\frac{1}{2(\rho+1)}+\frac{1}{2}=1$, então usando as desigualdades de Hölder e Young, existe uma constante $C>0$ tal que

$$
\begin{aligned}
\mid(f(u(t)) & \left.-f(v(t)), w_{t}(t)\right) \mid \\
& \leq \int_{\Omega}|f(u(t))-f(v(t))|\left|w_{t}(t)\right| d x \\
& \leq k_{2} \int_{\Omega}\left(1+|u(t)|^{\rho}+|v(t)|^{\rho}\right)|w(t)|\left|w_{t}(t)\right| d x \\
& \leq k_{2}\left(|\Omega|^{\frac{\rho}{2(\rho+1)}}+\|u(t)\|_{2(\rho+1)}^{\rho}+\|v(t)\|_{2(\rho+1)}^{\rho}\right)\|w(t)\|_{2(\rho+1)}\left\|w_{t}(t)\right\|_{2} \\
& \leq C\|\Delta w(t)\|_{2}^{2}+\frac{1}{2}\left\|w_{t}(t)\right\|_{2}^{2} .
\end{aligned}
$$

Combinando (2.50) com (2.51) e (2.52), resulta em

$$
\begin{gathered}
\frac{d}{d t}\left\{\left\|w_{t}(t)\right\|_{2}^{2}+\|\Delta w(t)\|_{2}^{2}-\left(\int_{0}^{t} g(s) d s\right)\|\nabla w(t)\|_{2}^{2}+(g \square \nabla w)(t)\right\} \\
+\left\|\nabla w_{t}(t)\right\|_{2}^{2} \leq C\|\Delta w(t)\|_{2}^{2}+\left\|w_{t}(t)\right\|_{2}^{2} .
\end{gathered}
$$

Pondo

$$
\Phi(t)=\left\|w_{t}(t)\right\|_{2}^{2}+\|\Delta w(t)\|_{2}^{2}-\left(\int_{0}^{t} g(s) d s\right)\|\nabla w(t)\|_{2}^{2}+(g \square \nabla w)(t)
$$

e usando a hipótese (2.5), então

$$
\|\Delta w(t)\|_{2}^{2}-\left(\int_{0}^{t} g(s) d s\right)\|\nabla w(t)\|_{2}^{2} \geq l\|\Delta w(t)\|_{2}^{2} \geq 0 .
$$

Como já é sabido,

$$
(g \square \nabla w)(t) \geq 0 .
$$

Assim, existe uma constante $C>0$ tal que (2.53) fica sob a forma

$$
\frac{d}{d t} \Phi(t) \leq C \Phi(t) .
$$

Como $\Phi(0)=0$, obtemos

$$
\left\|w_{t}(t)\right\|_{2}^{2}+l\|\Delta w(t)\|_{2}^{2} \leq 0,
$$


provando que $w=0$ em $H^{2}(\Omega) \cap H_{0}^{1}(\Omega)$ (também em $L^{2}(\Omega)$ ). Isto prova a unicidade.

A unicidade de solução fraca pode ser provada usando o método de regularização, como apresentado por Lions [66, Capítulo 1]. Essencialmente, usa-se as mesmas estimativas como no caso de soluções mais regulares, ver também Yang [96].

Portanto, a prova do Teorema 2.3.1 está completa.

\subsection{Decaimento exponencial de Energia}

A fim de determinar estabilidade exponencial para o sistema (2.1)-(2.3), vamos definir o funcional energia correspondente como

$$
E(t)=\frac{1}{2}\left\|u_{t}(t)\right\|_{2}^{2}+\frac{1}{2}\|\Delta u(t)\|_{2}^{2}+\frac{1}{p}\|\nabla u(t)\|_{p}^{p}+\int_{\Omega} \hat{f}(u(t)) d x
$$

Assim sendo, o principal resultado desta seção é o seguinte

Teorema 2.4.1. Sob as hipóteses do Teorema 2.3.1, a energia E(t) satisfaz (em ambos os casos) o seguinte decaimento exponencial

$$
E(t) \leq C E(0) e^{-\gamma t}, \quad t \geq 0,
$$

para constantes $C>0$ e $\gamma>0$.

Prova. Inicialmente provaremos o decaimento exponencial de energia considerando soluções mais regulares $u$ do problema (2.1)-(2.3). Usaremos o método de energia perturbada (ver por exemplo $[19,100])$

Em primeiro lugar, definamos a energia modificada

$$
\begin{aligned}
F(t)= & \frac{1}{2}\left\|u_{t}(t)\right\|_{2}^{2}+\frac{1}{2}\|\Delta u(t)\|_{2}^{2}+\frac{1}{p}\|\nabla u(t)\|_{p}^{p}+\int_{\Omega} \hat{f}(u(t)) d x \\
& -\frac{1}{2}\left(\int_{0}^{t} g(s) d s\right)\|\nabla u(t)\|_{2}^{2}+\frac{1}{2}(g \square \nabla u)(t) .
\end{aligned}
$$

Usando as hipóteses (2.5) e (2.9), vem que

$$
\begin{aligned}
F(t) & =E(t)-\frac{1}{2}\left(\int_{0}^{t} g(s) d s\right)\|\nabla u(t)\|_{2}^{2}+\frac{1}{2}(g \square \nabla u)(t) \\
& \geq l E(t) .
\end{aligned}
$$

Assim,

$$
E(t) \leq \frac{1}{l} F(t) .
$$

Além disso, da condição (2.6) segue que $F$ é decrescente, pois

$$
\begin{aligned}
F^{\prime}(t) & =-\left\|\nabla u_{t}(t)\right\|_{2}^{2}+\frac{1}{2}\left(g^{\prime} \square \nabla u\right)(t)-\frac{1}{2} g(t)\|\nabla u(t)\|_{2}^{2} \\
& \leq-\left\|\nabla u_{t}(t)\right\|_{2}^{2}-\frac{k_{1}}{2}(g \square \nabla u)(t) .
\end{aligned}
$$


Agora definamos a energia perturbada

$$
F_{\varepsilon}(t)=F(t)+\varepsilon \Psi(t), \quad \varepsilon>0,
$$

onde

$$
\Psi(t)=\int_{\Omega} u_{t}(t) u(t) d x .
$$

Com isto, demonstraremos dois lemas auxiliares que serão cruciais para a conclusão do decaimento exponencial.

Lema 2.4.2. Existe uma constante $C_{1}>0$ tal que

$$
\left|F_{\varepsilon}(t)-F(t)\right| \leq \varepsilon C_{1} F(t), \quad \forall t \geq 0, \forall \varepsilon>0 .
$$

Prova. Note que

$$
\begin{aligned}
|\Psi(t)| & \leq \frac{1}{2}\left\|u_{t}(t)\right\|_{2}^{2}+\frac{1}{2 \lambda_{1}}\|\Delta u(t)\|_{2}^{2} \\
& \leq \frac{1}{l} \max \left\{1, \frac{1}{\lambda_{1}}\right\} F(t),
\end{aligned}
$$

onde $\lambda_{1}>0$ é o primeiro autovalor do problema

$$
\left\{\begin{array}{lcc}
\Delta^{2} w=\lambda w & \text { em } & \Omega \\
w=\Delta w=0 & \text { sobre } & \Gamma .
\end{array}\right.
$$

Então tomando $C_{1}=\frac{1}{l} \max \left\{1, \frac{1}{\lambda_{1}}\right\}$, obtemos

$$
\left|F_{\varepsilon}(t)-F(t)\right|=\varepsilon|\Psi(t)| \leq \varepsilon C_{1} F(t),
$$

como desejado.

Lema 2.4.3. Existe uma constante $\varepsilon_{1}>0$ tal que

$$
F_{\varepsilon}^{\prime}(t) \leq-\varepsilon F(t), \quad \forall t \geq 0, \quad \forall \varepsilon \in\left(0, \varepsilon_{1}\right) .
$$

Prova. É suficiente mostrar que existem constantes $C_{2}, C_{3}>0$ tais que

$$
\Psi^{\prime}(t) \leq-F(t)+C_{2}\left\|\nabla u_{t}(t)\right\|_{2}^{2}+C_{3}(g \square \nabla u)(t) .
$$

De fato, para $\varepsilon>0$ pequeno o suficiente temos

$$
\begin{aligned}
F_{\varepsilon}^{\prime}(t) & =F^{\prime}(t)+\varepsilon \Psi^{\prime}(t) \\
& \leq-\left\|\nabla u_{t}(t)\right\|_{2}^{2}-\frac{k_{1}}{2}(g \square \nabla u)(t)-\varepsilon F(t)+\varepsilon C_{2}\left\|\nabla u_{t}(t)\right\|_{2}^{2}+\varepsilon C_{3}(g \square \nabla u)(t) \\
& =-\varepsilon F(t)-\left(1-\varepsilon C_{2}\right)\left\|\nabla u_{t}(t)\right\|_{2}^{2}-\left(k_{1} / 2-\varepsilon C_{3}\right)(g \square \nabla u)(t) \\
& \leq-\varepsilon F(t),
\end{aligned}
$$


o que concluiria a prova do Lema 2.4.3.

Resta verificar a veracidade da identidade (2.55). Com efeito, usando a equação (2.1) no sentido fraco, obtemos

$$
\begin{aligned}
\Psi^{\prime}(t)= & \left\|u_{t}(t)\right\|_{2}^{2}-\|\Delta u(t)\|_{2}^{2}-\|\nabla u(t)\|_{p}^{p}+\int_{0}^{t} g(t-s)(\nabla u(s), \nabla u(t)) d s \\
& -\int_{\Omega} \nabla u(t) \cdot \nabla u_{t}(t) d x-\int_{\Omega} f(u(t)) u(t) d x .
\end{aligned}
$$

Adicionando e subtraindo o termo $F(t)$ na expressão acima, segue que

$$
\begin{aligned}
\Psi^{\prime}(t)= & -F(t)+\frac{3}{2}\left\|u_{t}(t)\right\|_{2}^{2}-\frac{1}{2}\|\Delta u(t)\|_{2}^{2}-\left(1-\frac{1}{p}\right)\|\nabla u(t)\|_{p}^{p} \\
& -\frac{1}{2}\left(\int_{0}^{t} g(s) d s\right)\|\nabla u(t)\|_{2}^{2}+\frac{1}{2}(g \square \nabla u)(t)+I_{1}+I_{2}+I_{3},
\end{aligned}
$$

onde denotamos

$$
\begin{aligned}
I_{1} & =\int_{\Omega} \hat{f}(u(t))-f(u(t)) u(t) d x \\
I_{2} & =\int_{0}^{t} g(t-s)(\nabla u(s), \nabla u(t)) d s, \\
I_{3} & =-\int_{\Omega} \nabla u(t) \cdot \nabla u_{t}(t) d x .
\end{aligned}
$$

Da hipótese (2.9) temos $I_{1} \leq 0$ diretamente. Vamos estimar a seguir os termos $I_{2}$ e $I_{3}$.

Usando a desigualdade de Young, note que

$$
\begin{aligned}
\left|I_{2}\right| \leq & \int_{0}^{t} g(t-s)\|\nabla u(t)\|_{2}\left(\|\nabla u(t)-\nabla u(s)\|_{2}+\|\nabla u(t)\|_{2}\right) d s \\
\leq & \left(\int_{0}^{t} g(t-s) d s\right)\|\nabla u(t)\|_{2}^{2}+\|\nabla u(t)\|_{2} \int_{0}^{t} g(t-s)\|\nabla u(t)-\nabla u(s)\|_{2} d s \\
\leq & \left(\int_{0}^{t} g(s) d s\right)\|\nabla u(t)\|_{2}^{2}+\eta\|\nabla u(t)\|_{2}^{2}+\frac{1}{4 \eta}\|g\|_{1}(g \square \nabla u)(t) \\
\leq & \frac{1}{2}\left(\int_{0}^{t} g(s) d s\right)\|\nabla u(t)\|_{2}^{2}+\frac{\mu_{1}}{2}\left(\int_{0}^{t} g(s) d s\right)\|\Delta u(t)\|_{2}^{2} \\
& +\eta \mu_{1}\|\Delta u(t)\|_{2}^{2}+\frac{1}{4 \eta}\|g\|_{1}(g \square \nabla u)(t),
\end{aligned}
$$

onde $\eta>0$ será escolhido de modo conveniente mais adiante. Também pela desigualdade de Young, temos

$$
\begin{aligned}
\left|I_{3}\right| & \leq \eta\|\nabla u(t)\|_{2}^{2}+\frac{1}{4 \eta}\left\|\nabla u_{t}(t)\right\|_{2}^{2} \\
& \leq \eta \mu_{1}\|\Delta u(t)\|_{2}^{2}+\frac{1}{4 \eta}\left\|\nabla u_{t}(t)\right\|_{2}^{2} .
\end{aligned}
$$


Então,

$$
\begin{aligned}
\Psi^{\prime}(t) \leq & -F(t)+\left(\frac{3}{2} \mu_{2}+\frac{1}{4 \eta}\right)\left\|\nabla u_{t}(t)\right\|_{2}^{2}+\left(\frac{1}{2}+\frac{1}{4 \eta}\|g\|_{1}\right)(g \square \nabla u)(t) \\
& -\frac{1}{2}\left(1-\mu_{1} \int_{0}^{t} g(s) d s\right)\|\Delta u(t)\|_{2}^{2}+2 \eta \mu_{1}\|\Delta u(t)\|_{2}^{2} .
\end{aligned}
$$

Agora note que de (2.5), obtemos

$$
\Psi^{\prime}(t) \leq-F(t)+C_{2}\left\|\nabla u_{t}(t)\right\|_{2}^{2}+C_{3}(g \square \nabla u)(t)+\left(2 \eta \mu_{1}-\frac{l}{2}\right)\|\Delta u(t)\|_{2}^{2} .
$$

Logo, tomando $\eta>0$ pequeno o suficiente temos que a desigualdade (2.55) é verdadeira. Isto encerra a prova do Lema 2.4.3.

Agora, usarremos os lemas anteriores para concluir a propriedade de decaimento. Seja

$$
\varepsilon_{0}=\min \left\{\frac{1}{2 C_{1}}, \varepsilon_{1}\right\}
$$

Com isto, tomando $\varepsilon<\varepsilon_{0}$ segue do Lema 2.4.2 que

$$
\frac{1}{2} F(t) \leq F_{\varepsilon}(t) \leq \frac{3}{2} F(t), \quad t \geq 0 .
$$

A segunda desigualdade em (2.56) em conjunto com o Lema 2.4.3 implica que

$$
\frac{d}{d t} F_{\varepsilon}(t) \leq-\varepsilon F(t) \leq-\frac{2}{3} \varepsilon F_{\varepsilon}(t)
$$

Logo,

$$
F_{\varepsilon}(t) \leq F_{\varepsilon}(0) e^{-\frac{2}{3} \varepsilon t}
$$

Usando novamente (2.56) obtemos

$$
F(t) \leq 3 F(0) e^{-\frac{2}{3} \varepsilon t}
$$

Finalmente, comparando as funções $F$ e $E$, e notando que $F(0)=E(0)$, concluimos

$$
E(t) \leq \frac{3}{l} E(0) e^{-\frac{2}{3} \varepsilon t}, \quad t \geq 0 .
$$

Isto prova o decaimento exponencial de soluções mais regulares $u \in H_{\Gamma}^{3}(\Omega)$, como era desejado.

O mesmo resultado pode ser estendido para soluções fracas $u \in H^{2}(\Omega) \cap H_{0}^{1}(\Omega)$ usando argumentos de densidade, como em Cavalcanti et al [19].

Isto conclui a prova do Teorema 2.4.1. 



\section{CAPÍTULO \\ 3 \\ Um modelo de placas com $p$-Laplaciano e memória com história}

\subsection{Introdução}

Seja $\Omega$ um domínio limitado de $\mathbb{R}^{N}$ com fronteira suave $\Gamma=\partial \Omega$ e $\mathbb{R}^{+}=(0, \infty)$. Neste capítulo estudaremos o comportamento assintótico de soluções relativas ao seguinte problema de placas com perturbações não lineares e memória (com história para $t<0$ )

$$
u_{t t}+\alpha(0) \Delta^{2} u-\Delta_{p} u+\int_{0}^{\infty} \alpha^{\prime}(s) \Delta^{2} u(t-s) d s-\Delta u_{t}+f(u)=h \quad \text { em } \quad \Omega \times \mathbb{R}^{+},
$$

com condições de fronteira

$$
u=\Delta u=0 \quad \text { sobre } \quad \Gamma \times \mathbb{R}
$$

e condições iniciais

$$
u(x, \tau)=u_{0}(x, \tau) \quad \text { e } \quad u_{t}(x, \tau)=\partial_{t} u_{0}(x, \tau), \quad(x, \tau) \in \Omega \times(-\infty, 0],
$$

onde $\Delta_{p} u$ é o operador $p$-Laplaciano, $f(u)$ é uma não linearidade do tipo Lipschitz local e $h:=$ $h(x, t)$ é uma força externa. Também, $\alpha(0)>0$ e $\alpha^{\prime}(s) \leq 0$ para todo $s \in \mathbb{R}^{+}$. Aqui a função $u_{0}: \Omega \times(-\infty, 0] \rightarrow \mathbb{R}$ é caracterizada como história de $u$, sendo conhecida para valores negativos com respeito a variável $t$ correspondente ao tempo.

Para abordar o problema (3.1)-(3.3) vamos usar a ideia introduzida por Dafermos [30], acoplando uma nova variável ao problema, conhecida como história de deslocamento relativo (ver também 
Giorgi et al $[47,48,50])$. Mais precisamente, definamos

$$
\eta=\eta^{t}(x, s)=u(x, t)-u(x, t-s), \quad(x, s) \in \Omega \times \mathbb{R}^{+}, \quad t \geq 0 .
$$

Derivando (3.4) com respeito a $t$ e $s$, obtemos

$$
\eta_{t}^{t}(x, s)=-\eta_{s}^{t}(x, s)+u_{t}(x, t), \quad(x, s) \in \Omega \times \mathbb{R}^{+}, t \geq 0 .
$$

Além disso, note que para $t=0$, obtemos a condição inicial

$$
\eta^{0}(x, s)=u_{0}(x, 0)-u_{0}(x,-s), \quad(x, s) \in \Omega \times \mathbb{R}^{+} .
$$

Considerando, por simplicidade, $\mu(s)=-\alpha^{\prime}(s)$ e $\alpha(\infty)=1$, então usando (3.4) o problema original (3.1)-(3.3) pode ser convertido no seguinte sistema:

$$
\begin{aligned}
& u_{t t}+\Delta^{2} u-\Delta_{p} u+\int_{0}^{\infty} \mu(s) \Delta^{2} \eta^{t}(s) d s-\Delta u_{t}+f(u)=h \quad \text { em } \quad \Omega \times \mathbb{R}^{+}, \\
& \eta_{t}=-\eta_{s}+u_{t} \quad \text { em } \quad \Omega \times \mathbb{R}^{+} \times \mathbb{R}^{+},
\end{aligned}
$$

com condições de fronteira

$$
u=\Delta u=0 \quad \text { sobre } \quad \Gamma \times \mathbb{R}^{+}, \quad \eta=\Delta \eta=0 \quad \text { sobre } \quad \Gamma \times \mathbb{R}^{+} \times \mathbb{R}^{+},
$$

e condições iniciais

$$
u(x, 0)=u_{0}(x), u_{t}(x, 0)=u_{1}(x), \eta^{0}(x, s)=\eta_{0}(x, s),
$$

onde

$$
\left\{\begin{array}{l}
u_{0}(x)=u_{0}(x, 0), \quad x \in \Omega, \\
u_{1}(x)=\partial_{t} u_{0}(x, 0) \mid t=0, \quad x \in \Omega, \\
\eta_{0}(x, s)=u_{0}(x, 0)-u_{0}(x,-s), \quad(x, s) \in \Omega \times \mathbb{R}^{+}
\end{array}\right.
$$

Observação 3.1.1. Após fornecer as devidas hipóteses sobre o núcleo da memória $\mu$ e definir os espaços adequados para a história de deslocamento relativo $\eta$, segue que o problema (3.5)-(3.8) é equivalente ao problema original (3.1)-(3.3), ou seja, a partir das soluções de (3.5)-(3.8) obtemos soluções para (3.1)-(3.3) e reciprocamente. Isto pode ser feito de modo análogo ao mostrado por Grasseli \& Pata [52]. Sendo assim, por motivos de conveniência em nossas considerações futuras, vamos estudar com detalhes o problema (3.5)-(3.8).

Este capítulo está organizado como segue. Na seção 3.2 fixaremos as notações preliminares e as hipóteses precisas sobre os parâmetros do problema (3.5)-(3.8). Na seção 3.3 apresentaremos o resultado sobre existência de soluções via método de Faedo-Galerkin, separarando a demonstração em subseções para uma melhor leitura do texto. Na seção 3.4 mostraremos a existência de um conjunto absorvente para o sistema (3.5)-(3.8). Na seção 3.5 apresentaremos uma desigualdade de estabilização que será útil para determinar a existência de atratores. Por fim, na seção 3.6 mostraremos a existência de um atrator global com dimensão fractal finita para o semigrupo não linear gerado pelas soluções do problema (3.5)-(3.8). 


\subsection{Hipóteses e notações iniciais}

Inicialmente forneceremos as hipóteses sobre a constante $p$, sobre a função $f$ e também sobre o núcleo da memória $\mu$, presentes no problema (3.5)-(3.8).

Para $N \in \mathbb{N}$ assumiremos que:

$$
2 \leq p \leq \frac{2 N-2}{N-2} \quad \text { se } \quad N \geq 3 \quad \text { e } \quad p \geq 2 \quad \text { se } \quad N=1,2 .
$$

Com relação a função $f: \mathbb{R} \rightarrow \mathbb{R}$, asumiremos que:

$$
f(0)=0, \quad|f(u)-f(v)| \leq k_{0}\left(1+|u|^{\rho}+|v|^{\rho}\right)|u-v|, \quad \forall u, v \in \mathbb{R},
$$

onde $k_{0}>0$ é uma constante e

$$
0<\rho \leq \frac{4}{N-4} \quad \text { se } \quad N \geq 5 \quad \text { e } \quad \rho>0 \quad \text { se } \quad 1 \leq N \leq 4 .
$$

Ademais, suponhamos que

$$
-k_{1} \leq \hat{f}(u) \leq f(u) u, \quad \forall u \in \mathbb{R},
$$

onde $\hat{f}(z)=\int_{0}^{z} f(s) d s$ e $k_{1} \geq 0$.

Observação 3.2.1. Com a condição (3.9) segue do Teorema de Imersões de Sobolev, relembrado na seção 1.2 do Capítulo 1, que vale a seguinte cadeia de inclusões:

$$
H^{2}(\Omega) \cap H_{0}^{1}(\Omega) \hookrightarrow W_{0}^{1,2(p-1)}(\Omega) \hookrightarrow H_{0}^{1}(\Omega) \hookrightarrow L^{2}(\Omega) .
$$

Além disso, a condição (3.11) garante que

$$
H^{2}(\Omega) \cap H_{0}^{1}(\Omega) \hookrightarrow L^{2(\rho+1)}(\Omega) .
$$

Mais ainda, as condições (3.10) e (3.12) incluem funções da forma

$$
f(u) \approx|u|^{\rho} u-|u|^{\alpha} u, \quad 0<\alpha<\rho .
$$

Com respeito ao termo de memória, assumiremos que:

$$
\begin{gathered}
\mu \in C^{1}\left(\mathbb{R}^{+}\right) \cap L^{1}\left(\mathbb{R}^{+}\right), \quad \int_{0}^{\infty} \mu(s) d s=\mu_{0}>0, \\
\mu(s) \geq 0, \quad \mu^{\prime}(s) \leq 0, \quad \forall s \in \mathbb{R}^{+},
\end{gathered}
$$

e que existe uma constante $k_{2}>0$ tal que

$$
\mu^{\prime}(s)+k_{2} \mu(s) \leq 0, \quad \forall s \in \mathbb{R}^{+} .
$$

A seguir fixaremos as notações que serão usadas durante todo este capítulo. Consideremos, em primeiro lugar, os espaços

$$
V_{0}=L^{2}(\Omega), \quad V_{1}=H_{0}^{1}(\Omega), \quad V_{2}=H^{2}(\Omega) \cap H_{0}^{1}(\Omega)
$$


e

$$
V_{3}=\left\{u \in H^{3}(\Omega) \mid u=\Delta u=0 \text { sobre } \Gamma\right\},
$$

equipados com os respectivos produtos internos e normas

$$
\begin{array}{rll}
(u, v)_{V_{1}}=(\nabla u, \nabla v) & \text { e } & \|u\|_{V_{1}}=\|\nabla u\|_{2}, \\
(u, v)_{V_{2}}=(\Delta u, \Delta v) & \text { e } & \|u\|_{V_{2}}=\|\Delta u\|_{2}, \\
(u, v)_{V_{3}}=(\nabla \Delta u, \nabla \Delta v) & \text { e } & \|u\|_{V_{3}}=\|\nabla \Delta u\|_{2} .
\end{array}
$$

Como é padrão nesta tese, $(\cdot, \cdot)$ denota o produto interno em $L^{2}(\Omega)$ e $\|\cdot\|_{p}$ a norma em $L^{p}(\Omega)$. Quando não houver possibilidade de confusão, usaremos a mesma notação $\langle\cdot, \cdot\rangle$ para mencionar a dualidade entre os espaços e seus duais.

A fim de abordar a história de deslocamento relativo $\eta$ como uma nova variável, consideremos os seguintes espaços com peso $L_{\mu}^{2}$,

$$
\mathcal{M}_{i}:=L_{\mu}^{2}\left(\mathbb{R}^{+} ; V_{i}\right)=\left\{\xi: \mathbb{R}^{+} \rightarrow V_{i} \mid \int_{0}^{\infty} \mu(s)\|\xi(s)\|_{V_{i}}^{2} d s<\infty\right\}, \quad i=0,1,2,3,
$$

que são não vazios devido as hipóteses (3.13)-(3.14). Além disso, estes espaços são de Hilbert quando munido do produto interno e norma

$$
(\xi, \zeta)_{\mu, i}=\int_{0}^{\infty} \mu(s)(\xi(s), \zeta(s))_{V_{i}} d r, \quad\|\xi\|_{\mu, i}^{2}=\int_{0}^{\infty} \mu(s)\|\xi(s)\|_{V_{i}}^{2} d s, \quad i=0,1,2,3 .
$$

Finalmente, introduzimos os espaços de fase

$$
\mathcal{H}=V_{2} \times V_{0} \times \mathcal{M}_{2} \quad \text { e } \quad \mathcal{H}_{1}=V_{3} \times V_{1} \times \mathcal{M}_{3}
$$

equipados com as normas

$$
\|(u, v, \xi)\|_{\mathcal{H}}^{2}=\|\Delta u\|_{2}^{2}+\|v\|_{2}^{2}+\|\xi\|_{\mu, 2}^{2},
$$

$\mathrm{e}$

$$
\|(u, v, \xi)\|_{\mathcal{H}_{1}}^{2}=\|\nabla \Delta u\|_{2}^{2}+\|\nabla v\|_{2}^{2}+\|\xi\|_{\mu, 3}^{2},
$$

respectivamente.

\subsection{Existência, unicidade e dependência contínua}

Iniciamos com o conceito de solução fraca para o problema (3.5)-(3.8).

Definição 3.3.1. Dados $\left(u_{0}, u_{1}, \eta_{0}\right) \in \mathcal{H}$, diremos que uma função $z=\left(u, u_{t}, \eta\right) \in C([0, T], \mathcal{H})$ é uma solução fraca para o problema (3.5)-(3.8) se $z(0)=\left(u_{0}, u_{1}, \eta_{0}\right) \mathrm{e}$

$$
\begin{aligned}
& \frac{d}{d t}\left(u_{t}, \omega\right)+(\Delta u, \Delta \omega)+\left\langle|\nabla u|^{p-2} \nabla u, \nabla \omega\right\rangle \\
&+\int_{0}^{\infty} \mu(s)(\Delta \eta(s), \Delta \omega) d s+\left(\nabla u_{t}, \nabla \omega\right)+(f(u), \omega)=(h, \omega), \\
&\left(\partial_{t} \eta+\partial_{s} \eta, \xi\right)_{\mu, 2}=\left(u_{t}, \xi\right)_{\mu, 2},
\end{aligned}
$$

quase sempre em $[0, T]$, para todo $\operatorname{par}(\omega, \xi) \in V_{2} \times \mathcal{M}_{2}$. 
Para mostrar que o problema (3.5)-(3.8) é bem posto, temos o seguinte resultado

Teorema 3.3.1. Seja $h \in L^{2}\left(0, T ; L^{2}(\Omega)\right)$. Sob as condições (3.9)-(3.15), temos:

(i) Se $\left(u_{0}, u_{1}, \eta_{0}\right) \in \mathcal{H}$, então o problema (3.5)-(3.8) possui uma solução fraca

$$
\left(u, u_{t}, \eta\right) \in C([0, T], \mathcal{H}), \quad \forall T>0,
$$

satisfazendo

$$
u \in L^{\infty}\left(0, T ; V_{2}\right), \quad u_{t} \in L^{\infty}\left(0, T ; V_{0}\right) \cap L^{2}\left(0, T ; V_{1}\right), \quad \eta \in L^{\infty}\left(0, T ; \mathcal{M}_{2}\right) .
$$

(ii) Se $\left(u_{0}, u_{1}, \eta_{0}\right) \in \mathcal{H}_{1}$, então a solução fraca do problema (3.5)-(3.8) possui maior regularidade

$$
u \in L^{\infty}\left(0, T ; V_{3}\right), \quad u_{t} \in L^{\infty}\left(0, T ; V_{1}\right) \cap L^{2}\left(0, T ; V_{2}\right), \quad \eta \in L^{\infty}\left(0, T ; \mathcal{M}_{3}\right) .
$$

(iii) Em ambos os casos, a solução $\left(u, u_{t}, \eta\right)$ depende continuamente dos dados iniciais em $\mathcal{H}$. Mais precisamente, sejam $z_{i}=\left(u^{i}, u_{t}^{i}, \eta^{i}\right)$ duas soluções do problema (3.5)-(3.8) correspondentes aos dados iniciais $z_{i, 0}=\left(u_{0}^{i}, u_{1}^{i}, \eta_{0}^{i}\right)$, para $i=1,2$. Então, vale a seguinte estimativa:

$$
\left\|z_{1}(t)-z_{2}(t)\right\|_{\mathcal{H}} \leq e^{c t}|| z_{1,0}-z_{2,0} \|_{\mathcal{H}}, \quad t \in[0, T],
$$

para alguma constante $c>0$. Em particular, o problema (3.5)-(3.8) possui uma única solução fraca.

Prova. A existência de soluções será feita utilizando o método de Faedo-Galerkin com embasamento nos argumentos exibidos por Lions et al [66, 67] e Yang et al [96, 97]. Com respeito ao termo de memória seguiremos as ideias introduzidas por Dafermos [30, 31], as quais também foram expostas mais recentemente por Giorgi et al [47, 48, 50], entre outros autores. Para uma melhor compreensão do texto, separamos a demonstração em várias subseções como segue.

\subsubsection{Problema aproximado}

Seja $\left(\omega_{j}\right)_{j \in \mathbb{N}}$ a base de Galerkin dada por autofunções do problema $\Delta^{2} \omega=\lambda \omega$ em $\Omega$ com condições de fronteira $\omega=\Delta \omega=0$ sobre $\Gamma$. Então, sabe-se que a sequência $\left(\omega_{j}\right)_{j \in \mathbb{N}}$ pode ser escolhida de forma que seja ortogonal em $V_{1}=H_{0}^{1}(\Omega)$ e $V_{2}=H^{2}(\Omega) \cap H_{0}^{1}(\Omega)$, e ortonormal em $V_{0}=L^{2}(\Omega)$. Denotando por $\left(\lambda_{j}\right)_{j \in \mathbb{N}}$ a sequência de autovalores correspondentes, temos também:

$$
0<\lambda_{1} \leq \lambda_{2} \leq \cdots \leq \lambda_{j} \leq \cdots \quad \text { com } \quad \lambda_{j} \stackrel{j \rightarrow \infty}{\longrightarrow} \infty
$$

e

$$
\left\{\begin{array}{l}
\Delta^{2} \omega_{j}=\lambda_{j} \omega_{j} \quad \text { em } \quad \Omega, \\
\omega_{j}=\Delta \omega_{j}=0 \quad \text { sobre } \quad \Gamma, \quad j \in \mathbb{N} .
\end{array}\right.
$$

Mais ainda, devido a regularidade dos problemas elípticos e das imersões de Sobolev temos

$$
\omega_{j} \in\left(\bigcap_{m \in \mathbb{N}} H^{m}(\Omega)\right) \cap C^{\infty}(\bar{\Omega}) \cap H_{0}^{1}(\Omega), \quad j \in \mathbb{N} .
$$


A seguir vamos selecionar uma base ortonormal regular $\left(\xi_{j}\right)_{j \in \mathbb{N}}$ para o espaço $\mathcal{M}_{2}=$ $L_{\mu}^{2}\left(\mathbb{R}^{+} ; V_{2}\right)$. Para este fim prosseguiremos de modo análogo a Giorgi et al [48], tomando vetores (funções) $\xi_{j}$ da forma $\left(c_{i} \hat{\omega}_{k}\right)_{i, k \in \mathbb{N}}$, onde $\left(c_{i}\right)_{i \in \mathbb{N}}$ é uma base ortonormal de $L_{\mu}^{2}\left(\mathbb{R}^{+}\right) \cap C_{0}^{\infty}\left(\mathbb{R}^{+}\right) \mathrm{e}$ $\hat{\omega}_{k}=\frac{\omega_{k}}{\left\|\omega_{k}\right\|_{V_{2}}}$. De fato, se $\xi_{i}=c_{p} \hat{\omega}_{k}$ e $\xi_{j}=c_{q} \hat{\omega}_{l}$, então

$$
\left(\xi_{i}, \xi_{j}\right)_{\mu, 2}=\int_{0}^{\infty} \mu(s) c_{p}(s) c_{q}(s) d s\left(\hat{\omega}_{k}, \hat{\omega}_{l}\right)_{V_{2}}=\delta_{p q} \delta_{k l},
$$

de onde segue que $\left(\xi_{i}, \xi_{j}\right)_{\mu, 2}=\delta_{i j}$ (delta de Kronecker). Logo, $\left(\xi_{j}\right)_{j \in \mathbb{N}}$ é ortonormal em $\mathcal{M}_{2}$. Note ainda, que $\xi_{j} \in C_{0}^{\infty}\left(\mathbb{R}^{+}, V_{2}\right)$ para $j \in \mathbb{N}$.

Agora para cada $m \in \mathbb{N}$ consideremos os seguintes subespaços de dimensão finita

$$
W_{m}=\operatorname{Span}\left\{\omega_{1}, \ldots, \omega_{m}\right\} \subset V_{2} \quad \text { e } \quad Q_{m}=\operatorname{Span}\left\{\xi_{1}, \ldots, \xi_{m}\right\} \subset \mathcal{M}_{2}
$$

e, para dados iniciais $\left(u_{0}, u_{1}, \eta_{0}\right) \in \mathcal{H}$, procuraremos funções da forma

$$
u^{m}(t)=\sum_{k=1}^{m} y_{m k}(t) \omega_{k} \quad \text { e } \quad \eta^{t, m}(s)=\sum_{k=1}^{m} g_{m k}(t) \xi_{k}(s),
$$

que satisfaçam o seguinte problema aproximado

$$
\begin{aligned}
&\left(u_{t t}^{m}(t), \omega_{j}\right)+\left(\Delta u^{m}(t), \Delta \omega_{j}\right)-\left\langle\Delta_{p}\left(u^{m}(t)\right), \omega_{j}\right\rangle+\left(\eta^{t, m}, \omega_{j}\right)_{\mu, 2} \\
&-\left(\Delta u_{t}^{m}(t), \omega_{j}\right)+\left(f\left(u^{m}(t)\right), \omega_{j}\right)=\left(h(t), \omega_{j}\right), \\
&\left(\partial_{t} \eta^{t, m}, \xi_{j}\right)_{\mu, 2}=-\left(\partial_{s} \eta^{t, m}, \xi_{j}\right)_{\mu, 2}+\left(u_{t}^{m}(t), \xi_{j}\right)_{\mu, 2}, \quad j=1, \ldots, m
\end{aligned}
$$

com condições iniciais

$$
u^{m}(0)=u_{0}^{m}, \quad u_{t}^{m}(0)=u_{1}^{m}, \quad \eta^{0, m}=\eta_{0}^{m},
$$

onde

$$
u_{0}^{m} \rightarrow u_{0} \quad \text { em } \quad V_{2}, \quad u_{1}^{m} \rightarrow u_{1} \quad \text { em } \quad V_{0} \quad \text { e } \quad \eta_{0}^{m} \rightarrow \eta_{0} \quad \text { em } \quad \mathcal{M}_{2} .
$$

Devido a regularidade das funções aqui envolvidas, a notação $\langle\cdot, \cdot\rangle$ em (3.22), que denota a dualidade de $W^{-1, p^{\prime}}(\Omega)$ com $W_{0}^{1, p}(\Omega)$, podia ser substituida neste momento pela notação $(\cdot, \cdot)$ de produto interno em $V_{0}$. Contudo, a mesma será mantida por motivos de conveniência e para não haver confusão nos procedimentos futuros.

Note que o problema aproximado (3.22)-(3.24) pode ser reduzido no seguinte sistema de Equações Diferenciais Ordinárias (EDO) não lineares de segunda ordem, em $y_{m j}$ e $g_{m j}$, dado por

$$
\left\{\begin{array}{l}
y_{m j}^{\prime \prime}(t)+\lambda_{j}^{1 / 2} y_{m j}^{\prime}(t)+\lambda_{j} y_{m j}(t)+\sum_{k=1}^{m} g_{m k}(t)\left(\xi_{k}, \omega_{j}\right)_{\mu, 2}=H_{j}\left(t, y_{m j}(t)\right), \\
g_{m j}^{\prime}(t)=\sum_{k=1}^{m} y_{m k}^{\prime}(t)\left(\omega_{k}, \xi_{j}\right)_{\mu, 2}-\sum_{k=1}^{m} g_{m k}(t)\left(\partial_{s} \xi_{k}, \xi_{j}\right)_{\mu, 2} \\
y_{m j}(0)=\left(u_{0}^{m}, \omega_{j}\right), y_{m j}^{\prime}(0)=\left(u_{1}^{m}, \omega_{j}\right), g_{m j}(0)=\left(\eta_{0}^{m}, \xi_{j}\right)_{\mu, 2}, \quad j=1, \ldots, m,
\end{array}\right.
$$


onde a notação ' denota a derivada $\frac{d}{d t}$ e também

$$
H_{j}\left(t, y_{m j}(t)\right)=\left(h(t), \omega_{j}\right)+\Delta_{p}\left(y_{m j}(t)\right)-f\left(y_{m j}(t)\right), \quad j=1, \ldots, m,
$$

com

$$
\Delta_{p}\left(y_{m j}(t)\right)=\left\langle\Delta_{p}\left(\sum_{k=1}^{m} y_{m k}(t) \omega_{k}\right), \omega_{j}\right\rangle \text { e } f\left(y_{m j}(t)\right)=\left(f\left(\sum_{k=1}^{m} y_{m k}(t) \omega_{k}\right), \omega_{j}\right) .
$$

Além disso,

$$
\begin{gathered}
\sum_{j=1}^{m}\left(u_{0}^{m}, \omega_{j}\right) \omega_{j} \rightarrow u_{0} \quad \text { em } \quad V_{2}, \\
\sum_{j=1}^{m}\left(u_{1}^{m}, \omega_{j}\right) \omega_{j} \rightarrow u_{1} \quad \text { em } \quad V_{0}, \\
\sum_{j=1}^{m}\left(\eta_{0}^{m}, \xi_{j}\right)_{\mu, 2} \xi_{j} \rightarrow \eta_{0} \quad \text { em } \quad \mathcal{M}_{2} .
\end{gathered}
$$

Assim sendo, vamos determinar a existência de soluções para o sistema de EDO's (3.26) via teoria de geral para equações diferenciais ordinárias, ver Coddington \& Levinson [29]. Com efeito, reescrevendo o sistema (3.26) sob a forma matricial, obtemos

$$
\begin{aligned}
& {\left[\begin{array}{c}
y_{m 1}^{\prime \prime}(t) \\
\vdots \\
y_{m m}^{\prime \prime}(t)
\end{array}\right]=-\overbrace{\left[\begin{array}{ccc}
\lambda_{1}^{1 / 2} & \cdots & 0 \\
\vdots & \ddots & \vdots \\
0 & \cdots & \lambda_{m}^{1 / 2}
\end{array}\right]}^{C_{1}}\left[\begin{array}{c}
y_{m 1}^{\prime}(t) \\
\vdots \\
y_{m m}^{\prime}(t)
\end{array}\right]-\overbrace{\left[\begin{array}{ccc}
\lambda_{1} & \cdots & 0 \\
\vdots & \ddots & \vdots \\
0 & \cdots & \lambda_{m}
\end{array}\right]}^{C_{2}}\left[\begin{array}{c}
y_{m 1}(t) \\
\vdots \\
y_{m m}(t)
\end{array}\right]} \\
& \underbrace{\left[\begin{array}{ccc}
\left(\xi_{1}, \omega_{1}\right)_{\mu, 2} & \cdots & \left(\xi_{m}, \omega_{1}\right)_{\mu, 2} \\
\vdots & \ddots & \vdots \\
\left(\xi_{1}, \omega_{m}\right)_{\mu, 2} & \cdots & \left(\xi_{m}, \omega_{m}\right)_{\mu, 2}
\end{array}\right]}_{C_{3}}\left[\begin{array}{c}
g_{m 1}(t) \\
\vdots \\
g_{m m}(t)
\end{array}\right]+\left[\begin{array}{c}
H_{1}\left(t, y_{m 1}(t)\right) \\
\vdots \\
H_{m}\left(t, y_{m m}(t)\right)
\end{array}\right] \\
& {\left[\begin{array}{c}
g_{m 1}^{\prime}(t) \\
\vdots \\
g_{m m}^{\prime}(t)
\end{array}\right]=-\overbrace{\left[\begin{array}{ccc}
\left(\partial_{s} \xi_{1}, \xi_{1}\right)_{\mu, 2} & \cdots & \left(\partial_{s} \xi_{m}, \xi_{1}\right)_{\mu, 2} \\
\vdots & \ddots & \vdots \\
\left(\partial_{s} \xi_{1}, \xi_{m}\right)_{\mu, 2} & \cdots & \left(\partial_{s} \xi_{m}, \xi_{m}\right)_{\mu, 2}
\end{array}\right]}^{C_{4}}\left[\begin{array}{c}
g_{m 1}(t) \\
\vdots \\
g_{m m}(t)
\end{array}\right]} \\
& +\underbrace{\left[\begin{array}{ccc}
\left(\omega_{1}, \xi_{1}\right)_{\mu, 2} & \cdots & \left(\omega_{m}, \xi_{1}\right)_{\mu, 2} \\
\vdots & \ddots & \vdots \\
\left(\omega_{1}, \xi_{m}\right)_{\mu, 2} & \cdots & \left(\omega_{m}, \xi_{m}\right)_{\mu, 2}
\end{array}\right]}_{C_{5}}\left[\begin{array}{c}
y_{m 1}^{\prime}(t) \\
\vdots \\
y_{m m}^{\prime}(t)
\end{array}\right] \\
& {\left[\begin{array}{c}
y_{m 1}(0) \\
\vdots \\
y_{m m}(0)
\end{array}\right]=\left[\begin{array}{c}
\left(u_{0}^{m}, \omega_{1}\right) \\
\vdots \\
\left(u_{0}^{m}, \omega_{m}\right)
\end{array}\right],\left[\begin{array}{c}
y_{m 1}^{\prime}(0) \\
\vdots \\
y_{m m}^{\prime}(0)
\end{array}\right]=\left[\begin{array}{c}
\left(u_{1}^{m}, \omega_{1}\right) \\
\vdots \\
\left(u_{1}^{m}, \omega_{m}\right)
\end{array}\right],\left[\begin{array}{c}
g_{m 1}(0) \\
\vdots \\
g_{m m}(0)
\end{array}\right]=\left[\begin{array}{c}
\left(\eta_{0}^{m}, \xi_{1}\right)_{\mu, 2} \\
\vdots \\
\left(\eta_{0}^{m}, \xi_{m}\right)_{\mu, 2}
\end{array}\right] .}
\end{aligned}
$$


Pondo

$$
Y(t)=\left[\begin{array}{c}
y_{m 1}(t) \\
\vdots \\
y_{m m}(t)
\end{array}\right], \quad G(t)=\left[\begin{array}{c}
g_{m 1}(t) \\
\vdots \\
g_{m m}(t)
\end{array}\right] \quad \text { e } H(t, Y(t))=\left[\begin{array}{c}
H_{1}\left(y_{m 1}(t)\right) \\
\vdots \\
H_{m}\left(y_{m m}(t)\right)
\end{array}\right]
$$

reduzimos nossos estudos ao seguinte sistema não linear de EDO's

$$
\left\{\begin{array}{lcc}
Y^{\prime \prime}(t) & =C_{1} Y^{\prime}(t)+C_{2} Y(t)+C_{3} G(t)+H(t, Y(t)), \\
G^{\prime}(t) & =C_{4} G(t)+C_{5} Y^{\prime}(t), & 0<t \leq T,
\end{array}\right.
$$

com condições iniciais

$$
Y(0)=Y_{0}, \quad Y^{\prime}(0)=Y_{1} \quad \text { e } \quad G(0)=G_{0}
$$

onde

$$
Y_{0}=\left[\begin{array}{c}
\left(u_{0}^{m}, \omega_{1}\right) \\
\vdots \\
\left(u_{0}^{m}, \omega_{m}\right)
\end{array}\right], \quad Y_{1}=\left[\begin{array}{c}
\left(u_{1}^{m}, \omega_{1}\right) \\
\vdots \\
\left(u_{1}^{m}, \omega_{m}\right)
\end{array}\right] \quad \text { e } \quad G_{0}=\left[\begin{array}{c}
\left(\eta_{0}^{m}, \xi_{1}\right)_{\mu, 2} \\
\vdots \\
\left(\eta_{0}^{m}, \xi_{m}\right)_{\mu, 2}
\end{array}\right]
$$

Além disso, considerando

$$
X(t)=Y^{\prime}(t) \quad \text { e } \quad Z(t)=\left[\begin{array}{c}
Y(t) \\
X(t) \\
G(t)
\end{array}\right]
$$

vem que

$$
\begin{aligned}
Z^{\prime}(t) & =\left[\begin{array}{c}
X(t) \\
C_{1} X(t)+C_{2} Y(t)+C_{3} G(t) \\
C_{4} G(t)+C_{5} X(t)
\end{array}\right]+\left[\begin{array}{c}
0 \\
H(t, Y(t)) \\
0
\end{array}\right] \\
& =\underbrace{\left[\begin{array}{ccc}
0 & I_{m} & 0 \\
C_{2} & C_{1} & C_{3} \\
0 & C_{5} & C_{4}
\end{array}\right]}_{C} \cdot\left[\begin{array}{c}
Y(t) \\
X(t) \\
G(t)
\end{array}\right]+\underbrace{\left[\begin{array}{c}
0 \\
H(t, Y(t)) \\
0
\end{array}\right]}_{\Phi(t, Z(t))}, \\
Z(0) & =\left[\begin{array}{c}
Y_{0} \\
Y_{1} \\
G_{0}
\end{array}\right]:=Z_{0} .
\end{aligned}
$$

Oras, com isto podemos converter (3.29)-(3.30) no seguinte PVI de primeira ordem,

$$
\left\{\begin{array}{l}
Z^{\prime}(t)=C Z(t)+\Phi(t, Z(t)), \quad 0<t \leq T \\
Z(0)=Z_{0}
\end{array}\right.
$$


No que segue, provaremos que a função dada por

$$
\begin{aligned}
\varphi:[0, T] \times \mathbb{R}^{3 m} & \longrightarrow \mathbb{R}^{3 m} \\
(t, z) & \longmapsto \varphi(t, z)=C z+\Phi(t, z),
\end{aligned}
$$

satisfaz as condições do Teorema de Carathéodory (ver Teorema 1.1 em [29, Capítulo 2]).

De fato, inicialmente verificaremos que $\varphi$ é mensurável em $t$ para cada $z$ fixado e, também, contínua em $z$ para cada $t$ fixo. Antes disso, relembremos a expressão concreta para $\varphi$. Denotando $z=(y, x, g) \in \mathbb{R}^{3 m}$, com $y, x, g \in \mathbb{R}^{m}$, usando a expressão para $H(t, y)$ dada em (3.28) e a identidade (3.27), podemos reescrever $\Phi(t, z)$ do seguinte modo:

$$
\Phi(t, z)=\underbrace{\left[\begin{array}{c}
0 \\
P(y) \\
0
\end{array}\right]}_{\mathcal{P}(y)}+\underbrace{\left[\begin{array}{c}
0 \\
F(y) \\
0
\end{array}\right]}_{\mathcal{F}(y)}+\underbrace{\left[\begin{array}{c}
0 \\
\psi(t) \\
0
\end{array}\right]}_{\Psi(t)},
$$

onde

$$
\begin{gathered}
P(y)=\left[\begin{array}{c}
\left\langle\Delta_{p}\left(\sum_{k=1}^{m} y_{m k} \omega_{k}\right), \omega_{1}\right\rangle \\
\vdots \\
\left\langle\Delta_{p}\left(\sum_{k=1}^{m} y_{m k} \omega_{k}\right), \omega_{m}\right\rangle
\end{array}\right], F(y)=-\left[\begin{array}{c}
\left(f\left(\sum_{k=1}^{m} y_{m k} \omega_{k}\right), \omega_{1}\right) \\
\vdots
\end{array}\right] \\
\left.\mathrm{e} \quad \psi\left(f\left(\sum_{k=1}^{m} y_{m k} \omega_{k}\right), \omega_{m}\right)\right] \\
\psi\left[\begin{array}{c}
\left(h(t), \omega_{1}\right) \\
\vdots \\
\left(h(t), \omega_{m}\right)
\end{array}\right] .
\end{gathered}
$$

Assim sendo, $\varphi(t, z)$ fica sob a forma

$$
\varphi(t, z)=C z+\mathcal{P}(y)+\mathcal{F}(y)+\Psi(t)
$$

Como $h \in L^{2}\left(0, T ; L^{2}(\Omega)\right)$, então a aplicação

$$
t \rightarrow\left(h(t), \omega_{j}\right), \quad \forall j=1, \ldots, m,
$$

é mensurável e, consequentemente, a aplicação

$$
t \rightarrow \Psi(t)
$$

é mensurável. Logo, para quase todo $z \in \mathbb{R}^{3 m}$ fixado a função $\varphi$ dada em (3.32) é mensurável em $t$.

Agora provaremos a continuidade de $\varphi$ com relação a variável $z=(y, x, g) \in \mathbb{R}^{3 m}$. Para isto, consideramos $z_{n}=\left(y_{n}, x_{n}, g_{n}\right) \in \mathbb{R}^{3 m}$, com $y_{n}, x_{n}, g_{n} \in \mathbb{R}^{m}$, tal que

$$
z_{n}=\left(y_{n}, x_{n}, g_{n}\right) \stackrel{n \rightarrow \infty}{\longrightarrow}(y, x, g)=z \quad \text { em } \quad \mathbb{R}^{3 m} .
$$


Isto implica que

$$
y_{n} \stackrel{n \rightarrow \infty}{\longrightarrow} y \quad \text { em } \quad \mathbb{R}^{m} .
$$

Além disso, colocando $y_{n}=\left(y_{n, 1}, \ldots, y_{n, m}\right)$ e $y=\left(y_{1}, \ldots, y_{m}\right)$ em $\mathbb{R}^{m}$, temos em particular que

$$
y_{n, i} \stackrel{n \rightarrow \infty}{\longrightarrow} y_{i} \quad \text { em } \quad \mathbb{R}, \quad \forall i=1, \ldots, m
$$

De (3.34) e devido a regularidade da base $\left(\omega_{i}\right)_{i \in \mathbb{N}}$, segue que

$$
\sum_{i=1}^{m} y_{n, i} \omega_{i} \stackrel{n \rightarrow \infty}{\longrightarrow} \sum_{i=1}^{m} y_{i} \omega_{i} \quad \text { em } \quad W_{0}^{1, p}(\Omega)
$$

Conforme relembrado na Observação 1.3.1 (seção 1.3 do Capítulo 1), o operador $p$-Laplaciano $\Delta_{p}$ é monótono, hemicontínuo e limitado, de onde segue, assim como em Lions [66], que o operador $\Delta_{p}$ contínuo de $\left(W_{0}^{1, p}(\Omega), \tau_{\text {forte }}\right)$ em $\left(W^{-1, p^{\prime}}(\Omega), \tau_{\text {fraca* }}\right)$. Deste modo,

$$
\left\langle\Delta_{p}\left(\sum_{i=1}^{m} y_{n, i} \omega_{i}\right), \omega_{j}\right\rangle \stackrel{n \rightarrow \infty}{\longrightarrow}\left\langle\Delta_{p}\left(\sum_{i=1}^{m} y_{i} \omega_{i}\right), \omega_{j}\right\rangle, \quad \forall j=1, \ldots, m .
$$

Isto implica que

$$
\mathcal{P}\left(y_{n}\right) \stackrel{n \rightarrow \infty}{\longrightarrow} \mathcal{P}(y) .
$$

Por outro lado, note que de (3.34) temos também

$$
w_{n}:=\sum_{i=1}^{m} y_{n, i} \omega_{i} \stackrel{n \rightarrow \infty}{\longrightarrow} \sum_{i=1}^{m} y_{i} \omega_{i}:=w \quad \text { em } \quad C^{\infty}(\bar{\Omega}) \hookrightarrow L^{2}(\Omega) .
$$

Da hipótese (3.10) e novamente devido a regularidade de $\left(\omega_{i}\right)_{i \in \mathbb{N}}$, segue que

$$
\begin{aligned}
\left\|f\left(w_{n}\right)-f(w)\right\|_{2} & \leq k_{0}\left(1+\left\|w_{n}\right\|_{\infty}^{\rho}+\|w\|_{\infty}^{\rho}\right)\left\|w_{n}-w\right\|_{2} \\
& \leq K\left\|w_{n}-w\right\|_{2}
\end{aligned}
$$

Assim,

$$
f\left(\sum_{i=1}^{m} y_{n, i} \omega_{i}\right) \stackrel{n \rightarrow \infty}{\longrightarrow} f\left(\sum_{i=1}^{m} y_{i} \omega_{i}\right) \quad \text { em } \quad L^{2}(\Omega)
$$

e, consequentemente, obtemos

$$
\left(f\left(\sum_{i=1}^{m} y_{n, i} \omega_{i}\right), \omega_{j}\right) \stackrel{n \rightarrow \infty}{\longrightarrow}\left(f\left(\sum_{i=1}^{m} y_{i} \omega_{i}\right), \omega_{j}\right), \quad \forall j=1, \ldots, m,
$$

de onde segue que

$$
\mathcal{F}\left(y_{n}\right) \stackrel{n \rightarrow \infty}{\longrightarrow} \mathcal{F}(y)
$$

Agora, como consequência imediata de (3.33), obtemos a seguinte convergência

$$
C z_{n} \stackrel{n \rightarrow \infty}{\longrightarrow} C z .
$$


Portanto, para quase todo $t$ fixado, as convergências (3.35)-(3.38) nos permitem concluir que a função $\varphi$ dada em (3.32) é contínua na variável $z$, como queríamos.

Resta mostrar mais uma condição imposta no Teorema de Carathéodory, a saber, que existe uma função $m(t)$ integrável a Lebesgue tal que

$$
\|\varphi(t, z)\|_{\mathbb{R}^{3 m}} \leq m(t), \quad \forall(t, z) \in R,
$$

onde $R \subset[0, T] \times \mathbb{R}^{3 m}$ é um subconjunto compacto qualquer.

Com efeito, aproveitando as notações anteriores, note que para todo $(t, z)=(t, y, x, g) \in R \subset$ $[0, T] \times \mathbb{R}^{3 m}$, temos:

$$
\begin{aligned}
\|\varphi(t, z)\|_{\mathbb{R}^{3 m}} & =\|C z+\mathcal{P}(y)+\mathcal{F}(y)+\Psi(t)\|_{\mathbb{R}^{3 m}} \\
& \leq\|C z\|_{\mathbb{R}^{3 m}}+\|\mathcal{P}(y)\|_{\mathbb{R}^{3 m}}+\|\mathcal{F}(y)\|_{\mathbb{R}^{3 m}}+\|\Psi(t)\|_{\mathbb{R}^{3 m}} \\
& \leq|C|\|z\|_{\mathbb{R}^{3 m}}+\|P(y)\|_{\mathbb{R}^{m}}+\|F(y)\|_{\mathbb{R}^{m}}+\|\psi(t)\|_{\mathbb{R}^{m}} \\
& \leq c_{1}+\|P(y)\|_{\mathbb{R}^{m}}+\|F(y)\|_{\mathbb{R}^{m}}+\|\psi(t)\|_{\mathbb{R}^{m}},
\end{aligned}
$$

onde na última desigualdade usamos o fato que $\operatorname{proj}_{\mathbb{R}^{3 m}} R$ é um compacto de $\mathbb{R}^{3 m}$ e $z \in \operatorname{proj}_{\mathbb{R}^{3 m}} R$. No que segue, estimaremos os termos do lado direito da última desigualdade que surge em (3.40).

Iniciamos observando que proj $\mathbb{R}^{m} R$ é um compacto de $\mathbb{R}^{m}$ e sendo $y=\left(y_{1}, \ldots, y_{m}\right) \in \operatorname{proj}_{\mathbb{R}^{m}} R$, então existe uma constante $c_{R}$ tal que $\|y\|_{\mathbb{R}^{m}} \leq c_{R}$. Como $\left(\omega_{i}\right)_{i \in \mathbb{N}} \subset C^{\infty}(\bar{\Omega}) \hookrightarrow W_{0}^{1, p}(\Omega)$, então

$$
\begin{aligned}
\left\|\sum_{i=1}^{m} y_{i} \omega_{i}\right\|_{W_{0}^{1, p}(\Omega)} & \leq \sum_{i=1}^{m}\left|y_{i}\right|\left\|\omega_{i}\right\|_{W_{0}^{1, p}(\Omega)} \\
& \leq m \max \left\{\left\|\omega_{1}\right\|_{\infty}, \ldots,\left\|\omega_{m}\right\|_{\infty}\right\}\|y\|_{\mathbb{R}^{m}} \\
& \leq m \max \left\{\left\|\omega_{1}\right\|_{\infty}, \ldots,\left\|\omega_{m}\right\|_{\infty}\right\} c_{R}<\infty .
\end{aligned}
$$

Como o operador $\Delta_{p}$ leva limitados de $W_{0}^{1, p}(\Omega)$ em limitados de $W^{-1, p^{\prime}}(\Omega)$, segue que

$$
\left\|\Delta_{p}\left(\sum_{i=1}^{m} y_{i} \omega_{i}\right)\right\|_{W^{-1, p^{\prime}(\Omega)}} \leq c_{R}^{\prime} .
$$

Isto implica em

$$
\begin{aligned}
\|P(y)\|_{\mathbb{R}^{m}}^{2} & =\sum_{j=1}^{m}\left|\left\langle\Delta_{p}\left(\sum_{i=1}^{m} y_{i} \omega_{i}\right), \omega_{j}\right\rangle\right|^{2} \\
& \leq \sum_{j=1}^{m}\left\|\Delta_{p}\left(\sum_{i=1}^{m} y_{i} \omega_{i}\right)\right\|_{W^{-1, p^{\prime}}(\Omega)}^{2}\left\|\omega_{j}\right\|_{W_{0}^{1, p}(\Omega)}^{2} \\
& =\left\|\Delta_{p}\left(\sum_{i=1}^{m} y_{i} \omega_{i}\right)\right\|_{W^{-1, p^{\prime}}(\Omega)}^{2} \sum_{j=1}^{m}\left\|\omega_{j}\right\|_{W_{0}^{1, p}(\Omega)}^{2},
\end{aligned}
$$

ou seja,

$$
\|P(y)\|_{\mathbb{R}^{m}} \leq c_{R}^{\prime}\left(\sum_{j=1}^{m}\left\|\omega_{j}\right\|_{W_{0}^{1, p}(\Omega)}^{2}\right)^{1 / 2} \leq c_{2} .
$$


Prosseguindo, note também que

$$
\begin{aligned}
\|F(y)\|_{\mathbb{R}^{m}}^{2} & =\sum_{j=1}^{m}\left|\left(f\left(\sum_{i=1}^{m} y_{i} \omega_{i}\right), \omega_{j}\right)\right|^{2} \\
& \leq \sum_{j=1}^{m}\left\|f\left(\sum_{i=1}^{m} y_{i} \omega_{i}\right)\right\|_{2}^{2}\left\|\omega_{j}\right\|_{2}^{2} \\
& =\left\|f\left(\sum_{i=1}^{m} y_{i} \omega_{i}\right)\right\|_{2}^{2} \sum_{j=1}^{m}\left\|\omega_{j}\right\|_{2}^{2} .
\end{aligned}
$$

Como anteriormente, tomando $w=\sum_{i=1}^{m} y_{i} \omega_{i}$ e usando (3.36) com $w_{n} \equiv 0$, obtemos

$$
\begin{aligned}
\|F(y)\|_{\mathbb{R}^{m}} & \leq\|f(w)\|_{2}\left(\sum_{j=1}^{m}\left\|\omega_{j}\right\|_{2}^{2}\right)^{1 / 2} \\
& \leq K\|w\|_{2}\left(\sum_{j=1}^{m}\left\|\omega_{j}\right\|_{2}^{2}\right)^{1 / 2} \leq c_{3} .
\end{aligned}
$$

Observe ainda que

$$
\|\psi(t)\|_{\mathbb{R}^{m}}^{2}=\sum_{j=1}^{m}\left|\left(h(t), \omega_{j}\right)\right|^{2} \leq \sum_{j=1}^{m}\|h(t)\|_{2}^{2}\left\|\omega_{j}\right\|_{2}^{2}=\left(\sum_{j=1}^{m}\left\|\omega_{j}\right\|_{2}^{2}\right)\|h(t)\|_{2}^{2},
$$

o que implica em

$$
\|\psi(t)\|_{\mathbb{R}^{m}} \leq\left(\sum_{j=1}^{m}\left\|\omega_{j}\right\|_{2}^{2}\right)^{1 / 2}\|h(t)\|_{2} \leq c_{4}\|h(t)\|_{2} .
$$

Combinando as estimativas (3.41)-(3.43) com (3.40), concluimos que existe constante $c>0$ tal que

$$
\|\varphi(t, z)\|_{\mathbb{R}^{3 m}} \leq c\left(1+\|h(t)\|_{2}\right), \quad \forall(t, z) \in R
$$

Lembrando que $\|h(t)\|_{2} \in L^{2}(0, T)$, então a função $m(t):=c\left(1+\|h(t)\|_{2}\right)$ é integrável sobre $\operatorname{proj}_{t} R \subseteq[0, T]$ e, portanto, a condição (3.39) é satisfeita.

Pelo exposto acima, estamos em condições de aplicar o Teorema de Carathéodory (ver Coddington \& Levinson [29, Capítulo 2]). Fazendo isto, garantimos a existência de uma solução $Z(t)$ para o problema (3.31) em algum intervalo $\left[0, T_{m}\right)$ com $0<T_{m} \leq T$, sendo $Z(t)$ absolutamente contínua e derivável quase sempre em $\left[0, T_{m}\right)$. Logo, as funções $y_{m j}$ e $g_{m j}$, com $j=1, \ldots, m$, são soluções locais do sistema de $\operatorname{EDO}(3.26)$ em $\left[0, T_{m}\right)$. Consequentemente, para cada $m \in \mathbb{N}$, o problema aproximado (3.22)-(3.24) possui uma solução local $\left(u^{m}(t), \eta^{t, m}\right)$ em algum intervalo $\left[0, T_{m}\right)$ com $0<T_{m} \leq T$. Isto encerra esta primeira etapa. 


\subsubsection{Estimativa a priori 1}

A estimativa inicial que determinaremos abaixo nos permitirá estender as soluções locais $u^{m}(t)$ e $\eta^{t, m}$ ao intervalo [0,T], para qualquer $T>0$ dado. Além disso, essa estimativa a priori nos possibilitará extrair uma subsequência (de soluções) convergente para uma função, a qual será candidata a solução fraca do problema original.

Multiplicando as equações aproximadas (3.22) por $y_{m j}^{\prime}(t)$ e (3.23) por $g_{m j}(t)$ e somando com respeito a $j=1, \ldots, m$, implica em

$$
\begin{gathered}
\left(u_{t t}^{m}(t), u_{t}^{m}(t)\right)+\left(\Delta u^{m}(t), \Delta u_{t}^{m}(t)\right)-\left\langle\Delta_{p}\left(u^{m}(t)\right), u_{t}^{m}(t)\right\rangle+\left(\eta^{t, m}, u_{t}^{m}(t)\right)_{\mu, 2} \\
-\left(\Delta u_{t}^{m}(t), u_{t}^{m}(t)\right)+\left(f\left(u^{m}(t)\right), u_{t}^{m}(t)\right)=\left(h(t), u_{t}^{m}(t)\right) \\
\left(\partial_{t} \eta^{t, m}, \eta^{t, m}\right)_{\mu, 2}=-\left(\partial_{s} \eta^{t, m}, \eta^{t, m}\right)_{\mu, 2}+\left(u_{t}^{m}(t), \eta^{t, m}\right)_{\mu, 2}
\end{gathered}
$$

Notando que vale as seguintes identidades

$$
\begin{aligned}
\left(u_{t t}^{m}(t), u_{t}^{m}(t)\right) & =\frac{1}{2} \frac{d}{d t}\left\|u_{t}^{m}(t)\right\|_{2}^{2}, \\
\left(\Delta u^{m}(t), \Delta u_{t}^{m}(t)\right) & =\frac{1}{2} \frac{d}{d t}\left\|\Delta u^{m}(t)\right\|_{2}^{2}, \\
\left\langle-\Delta_{p}\left(u^{m}(t)\right), u_{t}^{m}(t)\right\rangle & =\frac{1}{p} \frac{d}{d t}\left\|\nabla u^{m}(t)\right\|_{p}^{p}, \\
\left(-\Delta u_{t}^{m}(t), u_{t}^{m}(t)\right) & =\left\|\nabla u_{t}^{m}(t)\right\|_{2}^{2}, \\
\left(f\left(u^{m}(t)\right), u_{t}^{m}(t)\right) & =\frac{d}{d t} \int_{\Omega} \hat{f}\left(u^{m}(t)\right) d x, \\
\left(\partial_{t} \eta^{t, m}, \eta^{t, m}\right)_{\mu, 2} & =\frac{1}{2} \frac{d}{d t}\left\|\eta^{t, m}\right\|_{\mu, 2}^{2},
\end{aligned}
$$

e substituindo (3.45) em (3.44), vem que

$$
\frac{1}{2} \frac{d}{d t} E^{m}(t)+\left\|\nabla u_{t}^{m}(t)\right\|_{2}^{2}+\left(\partial_{s} \eta^{t, m}, \eta^{t, m}\right)_{\mu, 2}=\left(h(t), u_{t}^{m}(t)\right)
$$

onde

$$
E^{m}(t)=\left\|u_{t}^{m}(t)\right\|_{2}^{2}+\left\|\Delta u^{m}(t)\right\|_{2}^{2}+\frac{2}{p}\left\|\nabla u^{m}(t)\right\|_{p}^{p}+\left\|\eta^{t, m}\right\|_{\mu, 2}^{2}+2 \int_{\Omega} \hat{f}\left(u^{m}(t)\right) d x
$$

Da condição (3.12),

$$
\int_{\Omega} \hat{f}\left(u_{t}^{m}(t)\right) d x \geq-k_{1}|\Omega|
$$

de onde segue que

$$
\left\|u_{t}^{m}(t)\right\|_{2}^{2}+\left\|\Delta u^{m}(t)\right\|_{2}^{2}+\left\|\eta^{t, m}\right\|_{\mu, 2}^{2} \leq E^{m}(t)+2 k_{1}|\Omega| .
$$


Por outro lado, das condições (3.13)-(3.15) e como $\eta^{t, m}(0)=0$, temos

$$
\begin{aligned}
\left(\partial_{s} \eta^{t, m}, \eta^{t, m}\right)_{\mu, 2} & =\int_{0}^{\infty} \mu(s)\left(\partial_{s}\left(\Delta \eta^{t, m}(s)\right), \Delta \eta^{t, m}(s)\right) d s \\
& =\frac{1}{2} \underbrace{\int_{0}^{\infty} \partial_{s}\left[\mu(s)\left\|\Delta \eta^{t, m}(s)\right\|_{2}^{2}\right] d s}_{=0}-\frac{1}{2} \int_{0}^{\infty} \mu^{\prime}(s)\left\|\Delta \eta^{t, m}(s)\right\|_{2}^{2} d s \\
& =-\frac{1}{2} \int_{0}^{\infty} \mu^{\prime}(s)\left\|\Delta \eta^{t, m}(s)\right\|_{2}^{2} d s \\
& \geq \frac{k_{2}}{2} \int_{0}^{\infty} \mu(s)\left\|\Delta \eta^{t, m}(s)\right\|_{2}^{2} d s \\
& =\frac{k_{2}}{2}\left\|\eta^{t, m}\right\|_{\mu, 2}^{2},
\end{aligned}
$$

ou seja,

$$
\left(\partial_{s} \eta^{t, m}, \eta^{t, m}\right)_{\mu, 2} \geq \frac{k_{2}}{2}\left\|\eta^{t, m}\right\|_{\mu, 2}^{2}
$$

Além disso, usando as desigualdades de Poincaré e Young,

$$
\left(h(t), u_{t}^{m}(t)\right) \leq d_{2}\|h(t)\|_{2}\left\|\nabla u_{t}^{m}(t)\right\|_{2} \leq \frac{1}{2}\left\|\nabla u_{t}^{m}(t)\right\|_{2}^{2}+\frac{d_{2}^{2}}{2}\|h(t)\|_{2}^{2},
$$

onde $d_{2}$ é a constante proveniente da imersão $V_{1} \hookrightarrow V_{0}$. Combinando (3.49)-(3.50) com (3.46) e omitindo termos não negativos, obtemos

$$
\frac{d}{d t} E^{m}(t)+\left\|\nabla u_{t}^{m}(t)\right\|_{2}^{2} \leq d_{2}^{2}\|h(t)\|_{2}^{2}
$$

Integrando (3.51) de 0 a $t, \operatorname{com} t \in\left[0, T_{m}\right)$, chegamos a

$$
E^{m}(t)+\int_{0}^{t}\left\|\nabla u_{t}^{m}(\tau)\right\|_{2}^{2} d \tau \leq E^{m}(0)+d_{2}^{2}\|h\|_{L^{2}\left(0, T ; L^{2}(\Omega)\right)}^{2} .
$$

Agora, combinando (3.48) e (3.52), resulta que

$$
\begin{aligned}
\left\|u_{t}^{m}(t)\right\|_{2}^{2}+\left\|\Delta u^{m}(t)\right\|_{2}^{2}+ & \left\|\eta^{t, m}\right\|_{\mu, 2}^{2}+\int_{0}^{t}\left\|\nabla u_{t}^{m}(\tau)\right\|_{2}^{2} d \tau \\
& \leq E^{m}(0)+d_{2}^{2}\|h\|_{L^{2}\left(0, T ; L^{2}(\Omega)\right)}^{2}+2 k_{1}|\Omega|
\end{aligned}
$$

Das hipóteses (3.10) e (3.12), note que

$$
\begin{aligned}
E^{m}(0) & =\left\|u_{1}^{m}\right\|_{2}^{2}+\left\|\Delta u_{0}^{m}\right\|_{2}^{2}+\frac{2}{p}\left\|\nabla u_{0}^{m}\right\|_{p}^{p}+\left\|\eta_{0}^{m}\right\|_{\mu, 2}^{2}+2 \int_{\Omega} \hat{f}\left(u_{0}^{m}\right) d x \\
& \leq\left\|u_{1}^{m}\right\|_{2}^{2}+\left\|\Delta u_{0}^{m}\right\|_{2}^{2}+\frac{2}{p}\left\|\nabla u_{0}^{m}\right\|_{p}^{p}+\left\|\eta_{0}^{m}\right\|_{\mu, 2}^{2}+2 \int_{\Omega} f\left(u_{0}^{m}\right) u_{0}^{m} d x \\
& \leq\left\|u_{1}^{m}\right\|_{2}^{2}+\left\|\Delta u_{0}^{m}\right\|_{2}^{2}+\frac{2}{p}\left\|\nabla u_{0}^{m}\right\|_{p}^{p}+\left\|\eta_{0}^{m}\right\|_{\mu, 2}^{2}+2\left\|f\left(u_{0}^{m}\right)\right\|\left\|_{2}\right\| u_{0}^{m} \|_{2} \\
& \leq\left\|u_{1}^{m}\right\|_{2}^{2}+\left\|\Delta u_{0}^{m}\right\|_{2}^{2}+\frac{2}{p}\left\|\nabla u_{0}^{m}\right\|_{p}^{p}+\left\|\eta_{0}^{m}\right\|_{\mu, 2}^{2}+\left\|u_{0}^{m}\right\|_{2}^{2}+\left\|f\left(u_{0}^{m}\right)\right\|_{2}^{2} \\
& \leq C\left(\left\|u_{1}^{m}\right\|_{2}^{2}+\left\|\Delta u_{0}^{m}\right\|_{2}^{2}+\left\|\nabla u_{0}^{m}\right\|_{p}^{p}+\left\|\eta_{0}^{m}\right\|_{\mu, 2}^{2}+\left\|u_{0}^{m}\right\|_{2}^{2}+\left\|u_{0}^{m}\right\|_{2(\rho+1)}^{2(\rho+1)}\right),
\end{aligned}
$$


onde $C=1+2 k_{0}^{2}$. Além disso, como

$$
H^{2}(\Omega) \cap H_{0}^{1}(\Omega) \hookrightarrow W_{0}^{1, p}(\Omega) \cap L^{2(\rho+1)}(\Omega) \cap L^{2}(\Omega),
$$

então usando as convergências (3.25) e a estimativa acima, existe uma constante $M_{1}>0$ tal que

$$
E^{m}(0)+d_{2}^{2}\|h\|_{L^{2}\left(0, T ; L^{2}(\Omega)\right)}^{2}+2 k_{1}|\Omega| \leq M_{1} .
$$

Logo, a estimativa (3.53) se reduz a

$$
\left\|u_{t}^{m}(t)\right\|_{2}^{2}+\left\|\Delta u^{m}(t)\right\|_{2}^{2}+\left\|\eta^{t, m}\right\|_{\mu, 2}^{2}+\int_{0}^{t}\left\|\nabla u_{t}^{m}(\tau)\right\|_{2}^{2} d \tau \leq M_{1},
$$

para todos $t \in\left[0, T_{m}\right)$ e $m \in \mathbb{N}$, onde $M_{1}>0$ é tal que

$$
M_{1}=M_{1}\left(\left\|u_{1}\right\|_{2},\left\|\Delta u_{0}\right\|_{2},\left\|\eta_{0}\right\|_{\mu, 2},\|h\|_{L^{2}\left(0, T ; L^{2}(\Omega)\right)},|\Omega|\right) .
$$

A estimativa (3.54) nos permite estender as soluções $u^{m}(t)$ e $\eta^{t, m}$ do problema aproximado (3.22)-(3.24) a todo intervalo $[0, T]$. Além disso, repetindo o mesmo procedimento anterior segue que (3.54) vale para todos $t \in[0, T]$ e $m \in \mathbb{N}$.

Portanto, a estimativa (3.54) implica que

$$
\begin{aligned}
& \left(u^{m}\right) \text { é limitada em } L^{\infty}\left(0, T ; V_{2}\right), \\
& \left(u_{t}^{m}\right) \text { é limitada em } L^{\infty}\left(0, T ; V_{0}\right) \cap L^{2}\left(0, T ; V_{1}\right), \\
& \left(\eta^{m}\right) \text { é limitada em } L^{\infty}\left(0, T ; \mathcal{M}_{2}\right) .
\end{aligned}
$$

\subsubsection{Passagem ao limite e solução fraca}

Em virtude de (3.55) e aplicando os Teoremas 1.2 .18 e 1.2.19, podemos extrair subsequências de $\left(u^{m}\right)$ e $\left(\eta^{m}\right)$, que ainda denotaremos por $\left(u^{m}\right)$ e $\left(\eta^{m}\right)$, tal que

$$
\begin{array}{clll}
u^{m} \stackrel{*}{*} u & \text { em } & L^{\infty}\left(0, T ; V_{2}\right), \\
u_{t}^{m} \stackrel{*}{\rightarrow} u_{t} & \text { em } & L^{\infty}\left(0, T ; V_{0}\right), \\
u_{t}^{m} \rightarrow u_{t} & \text { em } & L^{2}\left(0, T ; V_{1}\right), \\
\eta^{m} \stackrel{*}{\rightarrow} \eta & \text { em } & L^{\infty}\left(0, T ; \mathcal{M}_{2}\right) .
\end{array}
$$

Também, de modo análogo à convergência (2.23) provada no Capítulo 2, segue que

$$
u^{m} \rightarrow u \quad \text { em } \quad L^{2}\left(0, T ; V_{1}\right),
$$

ou melhor,

$$
u^{m} \rightarrow u \quad \text { em } \quad C\left([0, T], V_{1}\right),
$$

para todo $T>0$, ver ainda Observação 2.3.3 (ver também Kim [62] no caso abstrato).

De posse das convergências em (3.56)-(3.58), estamos aptos a proceder o limite no problema aproximado e determinar a existência de solução fraca desejada. Para tal feito, sejam $\theta, \vartheta \in \mathcal{D}(0, T)$ 
e $m, j \in \mathbb{N}$ com $m \geq j$. Multiplicando o problema aproximado (3.22) por $\theta$ e (3.23) por $\vartheta$ e, em seguida, integrando ambas expressões resultantes sobre $[0, T]$, obtemos

$$
\begin{array}{r}
-\int_{0}^{T}\left(u_{t}^{m}(t), \omega_{j}\right) \theta^{\prime}(t) d t+\int_{0}^{T}\left(\Delta u^{m}(t), \Delta \omega_{j}\right) \theta(t) d t-\int_{0}^{T}\left\langle\Delta_{p}\left(u^{m}(t)\right), \omega_{j}\right\rangle \theta(t) d t \\
+\int_{0}^{T}\left(\eta^{t, m}, \omega_{j}\right)_{\mu, 2} \theta(t) d t+\int_{0}^{T}\left(\nabla u_{t}^{m}(t), \nabla \omega_{j}\right) \theta(t) d t+\int_{0}^{T}\left(f\left(u^{m}(t)\right), \omega_{j}\right) \theta(t) d t \\
=\int_{0}^{T}\left(h(t), \omega_{j}\right) \theta(t) d t \\
-\int_{0}^{T}\left(\eta^{t, m}, \xi_{j}\right)_{\mu, 2} \vartheta^{\prime}(t) d t=-\int_{0}^{T}\left(\partial_{s} \eta^{t, m}, \xi_{j}\right)_{\mu, 2} \vartheta(t) d t-\int_{0}^{T}\left(u^{m}(t), \xi_{j}\right)_{\mu, 2} \vartheta^{\prime}(t) d t,
\end{array}
$$

onde usamos também integração por partes em (3.59) e (3.60). Agora note que das convergências obtidas em (3.56) segue que

$$
\begin{array}{cccc}
\int_{0}^{T}\left(u^{m}(t), v(t)\right) d t & \stackrel{m \rightarrow \infty}{\longrightarrow} & \int_{0}^{T}(u(t), v(t)) d t, \quad \forall v \in L^{1}\left(0, T ; V_{2}^{\prime}\right), \\
\int_{0}^{T}\left(u_{t}^{m}(t), w(t)\right) d t & \stackrel{m \rightarrow \infty}{\longrightarrow} & \int_{0}^{T}\left(u_{t}(t), w(t)\right) d t, \quad \forall w \in L^{1}\left(0, T ; V_{0}\right), \\
\int_{0}^{T}\left(u_{t}^{m}(t), y(t)\right) d t & \stackrel{m \rightarrow \infty}{\longrightarrow} & \int_{0}^{T}\left(u_{t}(t), y(t)\right) d t, \quad \forall y \in L^{2}\left(0, T ; V_{1}^{\prime}\right), \\
\int_{0}^{T}\left(\eta^{t, m}, \zeta^{t}\right) d t & \stackrel{m \rightarrow \infty}{\longrightarrow} & \int_{0}^{T}\left(\eta^{t}, \zeta^{t}\right) d t, \quad \forall \zeta \in L^{1}\left(0, T ; \mathcal{M}_{2}^{\prime}\right),
\end{array}
$$

onde no primeiro, terceiro e quarto limites acima,

$(\cdot, \cdot) \quad$ denota a dualidade $\langle\cdot, \cdot\rangle_{V_{2}, V_{2}^{\prime}}$

$(\cdot, \cdot) \quad$ denota a dualidade $\langle\cdot, \cdot\rangle_{V_{1}, V_{1}^{\prime}}$

$(\cdot, \cdot) \quad$ denota a dualidade $\langle\cdot, \cdot\rangle_{\mathcal{M}_{2}, \mathcal{M}_{2}^{\prime}}$,

respectivamente. Em particular, para

$$
\begin{gathered}
v=\left(\Delta^{2} \omega_{j}\right) \theta, \quad v=\left(\int_{0}^{\infty} \mu(s) \Delta^{2} \xi_{j}(s) d s\right) \vartheta^{\prime}, \quad w=\omega_{j} \theta^{\prime}, \\
y=-\left(\Delta \omega_{j}\right) \theta, \quad \zeta=\omega_{j} \theta \quad \text { e } \quad \zeta=\xi_{j} \vartheta^{\prime},
\end{gathered}
$$


segue diretamente das convergências acima que:

$$
\begin{array}{ccc}
\int_{0}^{T}\left(\Delta u^{m}(t), \Delta \omega_{j}\right) \theta(t) d t & \stackrel{m \rightarrow \infty}{\longrightarrow} & \int_{0}^{T}\left(\Delta u(t), \Delta \omega_{j}\right) \theta(t) d t, \\
\int_{0}^{T}\left(u^{m}(t), \xi_{j}\right)_{\mu, 2} \vartheta^{\prime}(t) d t & \stackrel{m \rightarrow \infty}{\longrightarrow} & \int_{0}^{T}\left(u(t), \xi_{j}\right)_{\mu, 2} \vartheta^{\prime}(t) d t, \\
\int_{0}^{T}\left(u_{t}^{m}(t), \omega_{j}\right) \theta^{\prime}(t) d t & \stackrel{m \rightarrow \infty}{\longrightarrow} & \int_{0}^{T}\left(u_{t}(t), \omega_{j}\right) \theta^{\prime}(t) d t, \\
\int_{0}^{T}\left(\nabla u_{t}^{m}(t), \nabla \omega_{j}\right) \theta(t) d t & \stackrel{m \rightarrow \infty}{\longrightarrow} & \int_{0}^{T}\left(\nabla u_{t}(t), \nabla \omega_{j}\right) \theta(t) d t, \\
\int_{0}^{T}\left(\eta^{t, m}, \omega_{j}\right)_{\mu, 2} \theta(t) d t & \stackrel{m \rightarrow \infty}{\longrightarrow} \int_{0}^{T}\left(\eta^{t}, \omega_{j}\right)_{\mu, 2} \theta(t) d t, \\
\int_{0}^{T}\left(\eta^{t, m}, \xi_{j}\right)_{\mu, 2} \vartheta^{\prime}(t) d t & \stackrel{m \rightarrow \infty}{\longrightarrow} \int_{0}^{T}\left(\eta^{t}, \xi_{j}\right)_{\mu, 2} \vartheta^{\prime}(t) d t .
\end{array}
$$

A seguir, vamos determinar o limite dos demais termos (também não lineares) presentes em (3.59)-(3.60). Mais precisamente, afirmamos que

$$
\begin{array}{ccc}
\int_{0}^{T}\left(\partial_{s} \eta^{t, m}, \xi_{j}\right)_{\mu, 2} \vartheta(t) d t & \stackrel{m \rightarrow \infty}{\longrightarrow} & \int_{0}^{T}\left(\partial_{s} \eta^{t}, \xi_{j}\right)_{\mu, 2} \vartheta(t) d t, \\
\int_{0}^{T}\left(f\left(u^{m}(t)\right), \omega_{j}\right) \theta(t) d t & \stackrel{m \rightarrow \infty}{\longrightarrow} \int_{0}^{T}\left(f(u(t)), \omega_{j}\right) \theta(t) d t, \\
\int_{0}^{T}\left\langle-\Delta_{p}\left(u^{m}(t)\right), \omega_{j}\right\rangle \theta(t) d t & \stackrel{m \rightarrow \infty}{\longrightarrow} \int_{0}^{T}\left\langle-\Delta_{p}(u(t)), \omega_{j}\right\rangle \theta(t) d t .
\end{array}
$$

No que segue, mostraremos o primeiro limite em (3.63), sendo os outros dois análogos aos já feito no capítulo anterior.

- Convergência do termo (3.63) . Usando integração por partes, notemos que

$$
\begin{aligned}
\left(\partial_{s} \eta^{t, m}, \xi_{j}\right)_{\mu, 2} & =\int_{0}^{\infty} \mu(s)\left(\Delta\left(\partial_{s} \eta^{t, m}(s)\right), \Delta \xi_{j}(s)\right) d s \\
& =\int_{0}^{\infty} \mu(s) \int_{\Omega} \partial_{s}\left(\Delta \eta^{t, m}(s)\right) \Delta \xi_{j}(s) d x d s \\
& =\int_{\Omega} \int_{0}^{\infty} \mu(s) \Delta \xi_{j}(s) \partial_{s}\left(\Delta \eta^{t, m}(s)\right) d s d x \\
& =-\int_{\Omega} \int_{0}^{\infty} \Delta \eta^{t, m}(s)\left[\mu^{\prime}(s) \Delta \xi_{j}(s)+\mu(s) \Delta\left(\partial_{s} \xi_{j}(s)\right)\right] d s d x \\
& =-\int_{0}^{\infty} \int_{\Omega}\left[\mu^{\prime}(s) \Delta \eta^{t, m}(s) \Delta \xi_{j}(s)+\mu(s) \Delta \eta^{t, m}(s) \Delta\left(\partial_{s} \xi_{j}(s)\right)\right] d x d s \\
& =-\int_{0}^{\infty}\left[\mu^{\prime}(s)\left(\Delta \eta^{t, m}(s), \Delta \xi_{j}(s)\right)+\mu(s)\left(\Delta \eta^{t, m}(s), \Delta\left(\partial_{s} \xi_{j}(s)\right)\right)\right] d s \\
& =-\int_{0}^{\infty} \mu^{\prime}(s)\left(\Delta \eta^{t, m}(s), \Delta \xi_{j}(s)\right) d s-\left(\eta^{t, m}, \partial_{s} \xi_{j}\right)_{\mu, 2} .
\end{aligned}
$$


Então,

$$
\begin{aligned}
\int_{0}^{T}\left(\partial_{s} \eta^{t, m}, \xi_{j}\right)_{\mu, 2} \vartheta(t) d t= & -\int_{0}^{T} \int_{0}^{\infty} \mu^{\prime}(s)\left(\Delta \eta^{t, m}(s), \Delta \xi_{j}(s)\right) d s \vartheta(t) d t \\
& -\int_{0}^{T}\left(\eta^{t, m}, \partial_{s} \xi_{j}\right)_{\mu, 2} \vartheta(t) d t .
\end{aligned}
$$

Como anteriormente, vamos tomar casos particulares de $\zeta$ e aplicar a convergência para $\eta$ dada anteriormente. Para isto, notamos em primeiro lugar que devido a regularidade de $\xi_{j}$, temos

$$
\xi_{j} \in L_{\frac{\left(\mu^{\prime}\right)^{2}}{\mu}}^{2}\left(\mathbb{R}^{+} ; V_{2}\right) \quad \text { e } \quad \partial_{s} \xi_{j} \in L_{\mu}^{2}\left(\mathbb{R}^{+} ; V_{2}\right), \quad j \in \mathbb{N} .
$$

Assim sendo, tomando $\zeta=\frac{\mu^{\prime}}{\mu} \xi_{j} \vartheta$ e $\zeta=\left(\partial_{s} \xi_{j}\right) \vartheta$, vem que

$$
\begin{array}{lll}
\int_{0}^{T}\left(\eta^{t, m}, \frac{\mu^{\prime}}{\mu} \xi_{j} \vartheta(t)\right)_{\mu, 2} d t & \stackrel{m \rightarrow \infty}{\longrightarrow} & \int_{0}^{T}\left(\eta^{t}, \frac{\mu^{\prime}}{\mu} \xi_{j} \vartheta(t)\right)_{\mu, 2} d t, \\
\int_{0}^{T}\left(\eta^{t, m}, \partial_{s} \xi_{j}\right)_{\mu, 2} \vartheta(t) d t & \stackrel{m \rightarrow \infty}{\longrightarrow} & \int_{0}^{T}\left(\eta^{t}, \partial_{s} \xi_{j}\right)_{\mu, 2} \vartheta(t) d t .
\end{array}
$$

Oras, desenvolvendo a expressão em (3.65) chegamos na seguinte convergência

$$
\int_{0}^{T} \int_{0}^{\infty} \mu^{\prime}(s)\left(\Delta \eta^{t, m}(s), \Delta \xi_{j}(s)\right) d s \vartheta(t) d t \stackrel{m \rightarrow \infty}{\longrightarrow} \int_{0}^{T} \int_{0}^{\infty} \mu^{\prime}(s)\left(\Delta \eta^{t}(s), \Delta \xi_{j}(s)\right) d s \vartheta(t) d t .
$$

Combinando (3.64) com (3.66)-(3.67), segue o limite em (3.63) $)_{1}$, como desejado.

- Convergência dos termos (3.63) $)_{2,3}$. Agora notamos que os outros dois limites em (3.63) são provados exatamente como feito em (2.27) e (2.29) na seção 2.3 do Capítulo 2.

Diante das convergências estabelecidas em (3.62)-(3.63), fixando $j$ e passando (3.59)-(3.60) ao limite quando $m \rightarrow \infty$, obtemos que o par $(u, \eta)$ satisfaz

$$
\begin{array}{r}
-\int_{0}^{T}\left(u_{t}(t), \omega_{j}\right) \theta^{\prime}(t) d t+\int_{0}^{T}\left(\Delta u(t), \Delta \omega_{j}\right) \theta(t) d t-\int_{0}^{T}\left\langle\Delta_{p}(u(t)), \omega_{j}\right\rangle \theta(t) d t \\
+\int_{0}^{T}\left(\eta^{t}, \omega_{j}\right)_{\mu, 2} \theta(t) d t+\int_{0}^{T}\left(\nabla u_{t}(t), \nabla \omega_{j}\right) \theta(t) d t+\int_{0}^{T}\left(f(u(t)), \omega_{j}\right) \theta(t) d t \\
=\int_{0}^{T}\left(h(t), \omega_{j}\right) \theta(t) d t \\
-\int_{0}^{T}\left(\eta^{t}, \xi_{j}\right)_{\mu, 2} \vartheta^{\prime}(t) d t=-\int_{0}^{T}\left(\partial_{s} \eta^{t}, \xi_{j}\right)_{\mu, 2} \vartheta(t) d t-\int_{0}^{T}\left(u(t), \xi_{j}\right)_{\mu, 2} \vartheta^{\prime}(t) d t
\end{array}
$$

ou seja, para todo $j \in \mathbb{N}$ e todos $\theta, \vartheta \in \mathcal{D}(0, T)$, vale que

$$
\begin{array}{r}
\int_{0}^{T} \frac{d}{d t}\left(u_{t}(t), \omega_{j}\right) \theta(t) d t+\int_{0}^{T}\left(\Delta u(t), \Delta \omega_{j}\right) \theta(t) d t-\int_{0}^{T}\left\langle\Delta_{p}(u(t)), \omega_{j}\right\rangle \theta(t) d t \\
+\int_{0}^{T}\left(\eta^{t}, \omega_{j}\right)_{\mu, 2} \theta(t) d t+\int_{0}^{T}\left(\nabla u_{t}(t), \nabla \omega_{j}\right) \theta(t) d t+\int_{0}^{T}\left(f(u(t)), \omega_{j}\right) \theta(t) d t \\
=\int_{0}^{T}\left(h(t), \omega_{j}\right) \theta(t) d t \\
\int_{0}^{T} \frac{d}{d t}\left(\eta^{t}, \xi_{j}\right)_{\mu, 2} \vartheta(t) d t=-\int_{0}^{T}\left(\partial_{s} \eta^{t}, \xi_{j}\right)_{\mu, 2} \vartheta(t) d t+\int_{0}^{T} \frac{d}{d t}\left(u(t), \xi_{j}\right)_{\mu, 2} \vartheta(t) d t
\end{array}
$$


Como $\left(\omega_{j}\right)_{j \in \mathbb{N}}$ e $\left(\xi_{j}\right)_{j \in \mathbb{N}}$ constituem bases de $V_{2}$ e $\mathcal{M}_{2}$, respectivamente, então

$$
\begin{array}{r}
\int_{0}^{T} \frac{d}{d t}\left(u_{t}(t), \omega\right) \theta(t) d t+\int_{0}^{T}(\Delta u(t), \Delta \omega) \theta(t) d t-\int_{0}^{T}\left\langle\Delta_{p}(u(t)), \omega\right\rangle \theta(t) d t \\
+\int_{0}^{T}\left(\eta^{t}, \omega\right)_{\mu, 2} \theta(t) d t+\int_{0}^{T}\left(\nabla u_{t}(t), \nabla \omega\right) \theta(t) d t+\int_{0}^{T}(f(u(t)), \omega) \theta(t) d t \\
=\int_{0}^{T}(h(t), \omega) \theta(t) d t \\
\int_{0}^{T} \frac{d}{d t}\left(\eta^{t}, \xi\right)_{\mu, 2} \vartheta(t) d t=-\int_{0}^{T}\left(\partial_{s} \eta^{t}, \xi\right)_{\mu, 2} \vartheta(t) d t+\int_{0}^{T} \frac{d}{d t}(u(t), \xi)_{\mu, 2} \vartheta(t) d t
\end{array}
$$

para todos

$$
(\omega, \xi) \in V_{2} \times \mathcal{M}_{2} \quad \text { e } \quad \theta, \vartheta \in \mathcal{D}(0, T) .
$$

Agora note que podemos reescrever (3.68)-(3.69) como

$$
\begin{aligned}
& \left\langle\frac{d}{d t}\left(u_{t}, \omega\right)+(\Delta u, \Delta \omega)-\left\langle\Delta_{p}(u), \omega\right\rangle+(\eta, \omega)_{\mu, 2}+\left(\nabla u_{t}, \nabla \omega\right)+(f(u), \omega), \theta\right\rangle=\langle(h, \omega), \theta\rangle, \\
& \left\langle\frac{d}{d t}(\eta, \xi)_{\mu, 2}, \vartheta\right\rangle=\left\langle-\left(\partial_{s} \eta, \xi\right)_{\mu, 2}+\frac{d}{d t}(u, \xi)_{\mu, 2}, \vartheta\right\rangle, \quad \forall \theta, \vartheta \in \mathcal{D}(0, T),
\end{aligned}
$$

de onde segue que

$$
\begin{aligned}
& \frac{d}{d t}\left(u_{t}, \omega\right)+(\Delta u, \Delta \omega)-\left\langle\Delta_{p}(u), \omega\right\rangle+(\eta, \omega)_{\mu, 2}+\left(\nabla u_{t}, \nabla \omega\right)+(f(u), \omega)=(h, \omega), \\
& \frac{d}{d t}(\eta, \xi)_{\mu, 2}=-\left(\partial_{s} \eta, \xi\right)_{\mu, 2}+\frac{d}{d t}(u, \xi)_{\mu, 2},
\end{aligned}
$$

em $\mathcal{D}^{\prime}(0, T)$ para todo par $(\omega, \xi) \in V_{2} \times \mathcal{M}_{2}$. Isto mostra que a função $z:=\left(u, u_{t}, \eta\right)$ satisfaz (3.16)-(3.17) com

$$
\begin{aligned}
& u \in L^{\infty}\left(0, T ; V_{2}\right), \\
& u_{t} \in L^{\infty}\left(0, T ; V_{0}\right) \cap L^{2}\left(0, T ; V_{1}\right), \\
& \eta \in L^{\infty}\left(0, T ; \mathcal{M}_{2}\right),
\end{aligned}
$$

o que também prova a condição (3.18).

Para concluir que o limite $z=\left(u, u_{t}, \eta\right)$ é, de fato, uma solução fraca do problema (3.5)-(3.8) resta verificar que

$$
z \in C([0, T], \mathcal{H}) \quad \text { e } \quad z(0)=\left(u_{0}, u_{1}, \eta_{0}\right) .
$$

Contudo, a continuidade de $u, u_{t}$ em $[0, T]$ segue de modo análogo a Yang \& Jin [98]. Além disso, a continuidade de $\eta$ segue de modo similar como provado em Pata \& Zucchi [83], sendo uma consequência do fato de que o operador de derivação $-\partial_{s}$ gera um $C_{0}$-semigrupo de contrações em $\mathcal{M}_{2}$. Estas continuidades serão comprovadas mais adiante, em um só passo, para funções mais regulares. Depois, pelo uso de sequências regularizantes segue também a continuidade de solução fraca. Assim sendo, deduzimos que a requerida continuidade de $z$ vale. 
Com isto, $z(0)=\left(u(0), u_{t}(0), \eta^{0}\right)$ faz sentido e a condição inicial $z(0)=\left(u_{0}, u_{1}, \eta_{0}\right)$ pode ser verificada fazendo escolhas convenientes para as funções testes. Para tal feito, consideremos $\theta, \vartheta \in C^{1}([0, T]) \operatorname{com} \theta(0)=\vartheta(0)=1$ e $\theta(T)=\vartheta(T)=0$.

Provaremos inicialmente que $u(0)=u_{0}$. De fato, para $m, j \in \mathbb{N}$ com $m \geq j$, vem de (3.61) 1 $\operatorname{com} v=\omega_{j} \theta^{\prime}$ e de $(3.61)_{3} \operatorname{com} w=\omega_{j} \theta$, que

$$
\begin{array}{ccc}
\int_{0}^{T}\left(u^{m}(t), \omega_{j}\right) \theta^{\prime}(t) d t & \stackrel{m \rightarrow \infty}{\longrightarrow} & \int_{0}^{T}\left(u(t), \omega_{j}\right) \theta^{\prime}(t) d t, \\
\int_{0}^{T}\left(u_{t}^{m}(t), \omega_{j}\right) \theta(t) d t & \stackrel{m \rightarrow \infty}{\longrightarrow} & \int_{0}^{T}\left(u_{t}(t), \omega_{j}\right) \theta(t) d t,
\end{array}
$$

respectivamente. Usando integração por partes em (3.72) resulta

$$
-\left(u^{m}(0), \omega_{j}\right)-\int_{0}^{T}\left(u^{m}(t), \omega_{j}\right) \theta^{\prime}(t) d t \stackrel{m \rightarrow \infty}{\longrightarrow}-\left(u(0), \omega_{j}\right)-\int_{0}^{T}\left(u(t), \omega_{j}\right) \theta^{\prime}(t) d t
$$

e combinando isto com (3.71) segue que

$$
\left(u^{m}(0), \omega_{j}\right) \stackrel{m \rightarrow \infty}{\longrightarrow}\left(u(0), \omega_{j}\right), \quad \forall j \in \mathbb{N},
$$

o que implica na seguinte convergência

$$
u^{m}(0) \rightarrow u(0) \quad \text { em } \quad L^{2}(\Omega) .
$$

Por outro lado, da primeira convergência em (3.25) temos

$$
u^{m}(0) \rightarrow u_{0} \quad \text { em } \quad L^{2}(\Omega) .
$$

Portanto, da unicidade do limite fraco obtemos que $u(0)=u_{0}$.

Agora provaremos que $u_{t}(0)=u_{1}$. Com efeito, multiplicando o problema aproximado (3.22) por $\theta$, integrando sobre $[0, T]$ e usando integração por partes, obtemos

$$
\begin{aligned}
-\left(u_{t}^{m}(0), \omega_{j}\right)-\int_{0}^{T}\left(u_{t}^{m}(t), \omega_{j}\right) \theta^{\prime}(t) d t+\int_{0}^{T}\left(\Delta u^{m}(t), \Delta \omega_{j}\right) \theta(t) d t \\
-\int_{0}^{T}\left\langle\Delta_{p}\left(u^{m}(t)\right), \omega_{j}\right\rangle \theta(t) d t+\int_{0}^{T}\left(\eta^{t, m}, \omega_{j}\right)_{\mu, 2} \theta(t) d t \\
+\int_{0}^{T}\left(\nabla u_{t}^{m}(t), \nabla \omega_{j}\right) \theta(t) d t+\int_{0}^{T}\left(f\left(u^{m}(t)\right), \omega_{j}\right) \theta(t) d t=\int_{0}^{T}\left(h(t), \omega_{j}\right) \theta(t) d t .
\end{aligned}
$$

Agora note que da segunda convergência em (3.25),

$$
\left(u_{t}^{m}(0), \omega_{j}\right) \stackrel{m \rightarrow \infty}{\longrightarrow}\left(u_{1}, \omega_{j}\right), \quad \forall j \in \mathbb{N} .
$$

Logo, tomando limite quando $m \rightarrow \infty$ e levando em conta as convergências (3.62)-(3.63), mais o fato de que $\left(\omega_{j}\right)_{j \in \mathbb{N}}$ constitui uma base para $V_{2}$, então

$$
\begin{aligned}
-\left(u_{1}, \omega\right) & -\int_{0}^{T}\left(u_{t}(t), \omega\right) \theta^{\prime}(t) d t+\int_{0}^{T}(\Delta u(t), \Delta \omega) \theta(t) d t \\
& -\int_{0}^{T}\left\langle\Delta_{p}(u(t)), \omega\right\rangle \theta(t) d t+\int_{0}^{T}\left(\eta^{t}, \omega\right)_{\mu, 2} \theta(t) d t \\
& +\int_{0}^{T}\left(\nabla u_{t}(t), \nabla \omega\right) \theta(t) d t+\int_{0}^{T}(f(u(t)), \omega) \theta(t) d t=\int_{0}^{T}(h(t), \omega) \theta(t) d t,
\end{aligned}
$$


para todo $\omega \in V_{2}$. Novamente usando integração por partes, vem que

$$
\begin{aligned}
-\left(u_{1}, \omega\right) & +\left(u_{t}(0), \omega\right)+\int_{0}^{T} \frac{d}{d t}\left(u_{t}(t), \omega\right) \theta(t) d t+\int_{0}^{T}(\Delta u(t), \Delta \omega) \theta(t) d t \\
& -\int_{0}^{T}\left\langle\Delta_{p}(u(t)), \omega\right\rangle \theta(t) d t+\int_{0}^{T}\left(\eta^{t}, \omega\right)_{\mu, 2} \theta(t) d t \\
& +\int_{0}^{T}\left(\nabla u_{t}(t), \nabla \omega\right) \theta(t) d t+\int_{0}^{T}(f(u(t)), \omega) \theta(t) d t=\int_{0}^{T}(h(t), \omega) \theta(t) d t .
\end{aligned}
$$

Comparando (3.68) com (3.73), resulta que

$$
\left(u_{t}(0), \omega\right)=\left(u_{1}, \omega\right), \quad \forall \omega \in V_{2} .
$$

Portanto, pela densidade de $V_{2}=H^{2}(\Omega) \cap H_{0}^{1}(\Omega)$ em $V_{0}=L^{2}(\Omega)$, obtemos que $u_{t}(0)=u_{1}$.

Resta mostrar que $\eta^{0}=\eta_{0}$. Veremos a seguir que este caso segue de modo análogo ao anterior, devendo apenas substituir as variáveis e os espaços em questão. De fato, multiplicando o problema aproximado (3.23) por $\vartheta$, integrando sobre $[0, T]$ e usando integração por partes, obtemos

$$
\begin{aligned}
& -\left(\eta^{0, m}, \xi_{j}\right)_{\mu, 2}-\int_{0}^{T}\left(\eta^{t, m}, \xi_{j}\right)_{\mu, 2} \vartheta^{\prime}(t) d t \\
& =-\int_{0}^{T}\left(\partial_{s} \eta^{t, m}, \xi_{j}\right)_{\mu, 2} \vartheta(t) d t-\left(u^{m}(0), \xi_{j}\right)_{\mu, 2}-\int_{0}^{T}\left(u^{m}(t), \xi_{j}\right)_{\mu, 2} \vartheta^{\prime}(t) d t,
\end{aligned}
$$

Como anteriormente, segue das convergências em (3.25) 1 e (3.25) 3 que

$$
\begin{aligned}
\left(u^{m}(0), \xi_{j}\right)_{\mu, 2} & \stackrel{m \rightarrow \infty}{\longrightarrow}\left(u_{0}, \xi_{j}\right)_{\mu, 2}, \\
\left(\eta^{0, m}, \xi_{j}\right)_{\mu, 2} & \stackrel{m \rightarrow \infty}{\longrightarrow}\left(\eta_{0}, \xi_{j}\right)_{\mu, 2}, \quad \forall j \in \mathbb{N} .
\end{aligned}
$$

Assim, tomando limite quando $m \rightarrow \infty$, usando algumas das convergências (3.62)-(3.63) e o fato de que $\left(\xi_{j}\right)_{j \in \mathbb{N}}$ constitui uma base para $\mathcal{M}_{2}$, então

$$
\begin{aligned}
-\left(\eta_{0}, \xi\right)_{\mu, 2} & -\int_{0}^{T}\left(\eta^{t}, \xi\right)_{\mu, 2} \vartheta^{\prime}(t) d t \\
& =-\int_{0}^{T}\left(\partial_{s} \eta^{t}, \xi\right)_{\mu, 2} \vartheta(t) d t-\left(u_{0}, \xi\right)_{\mu, 2}-\int_{0}^{T}(u(t), \xi)_{\mu, 2} \vartheta^{\prime}(t) d t,
\end{aligned}
$$

para todo $\xi \in \mathcal{M}_{2}$, ou ainda,

$$
\begin{aligned}
& -\left(\eta_{0}, \xi\right)_{\mu, 2}+\left(\eta^{0}, \xi\right)_{\mu, 2}+\int_{0}^{T} \frac{d}{d t}\left(\eta^{t}, \xi\right)_{\mu, 2} \vartheta(t) d t \\
& =-\int_{0}^{T}\left(\partial_{s} \eta^{t}, \xi\right)_{\mu, 2} \vartheta(t) d t+\left(u(0)-u_{0}, \xi\right)_{\mu, 2}+\int_{0}^{T} \frac{d}{d t}(u(t), \xi)_{\mu, 2} \vartheta(t) d t .
\end{aligned}
$$

Comparando (3.69) com (3.74) e como $u(0)=u_{0}$, resulta que

$$
\left(\eta^{0}, \xi\right)_{\mu, 2}=\left(\eta_{0}, \xi\right)_{\mu, 2}, \quad \forall \xi \in \mathcal{M}_{2}
$$

donde segue que $\eta^{0}=\eta_{0}$, como era desejado. Isto mostra que a função $z=\left(u, u_{t}, \eta\right)$ é uma solução fraca de (3.5)-(3.8). 
Observação 3.3.1. Além do exposto acima, verificaremos também que:

$$
\begin{aligned}
u_{t t} & \in L^{2}\left(0, T ; V_{2}^{\prime}\right), \\
\eta_{t}+\eta_{s} & \in L^{\infty}\left(0, T ; \mathcal{M}_{0}\right) \cap L^{2}\left(0, T ; \mathcal{M}_{1}\right) .
\end{aligned}
$$

De fato, identificando $V_{0}$ com seu dual, por meio do Teorema de Representação de Riesz-Fréchet, então vale as seguintes cadeias de inclusões contínuas

$$
V_{2} \hookrightarrow W_{0}^{1, p} \hookrightarrow V_{1} \hookrightarrow V_{0} \hookrightarrow V_{1}^{\prime} \hookrightarrow W^{-1, p^{\prime}} \hookrightarrow V_{2}^{\prime} \quad \text { e } \quad \mathcal{M}_{2} \hookrightarrow \mathcal{M}_{0} \hookrightarrow \mathcal{M}_{2}^{\prime} .
$$

Em virtude desta identificação podemos reescrever (3.68)-(3.69) como

$$
\begin{gathered}
-\left\langle\int_{0}^{T} u_{t}(t) \theta^{\prime}(t) d t, \omega\right\rangle+\left\langle\int_{0}^{T} \Delta^{2} u(t) \theta(t) d t, \omega\right\rangle-\left\langle\int_{0}^{T} \Delta_{p} u(t) \theta(t) d t, \omega\right\rangle \\
+\left\langle\int_{0}^{T}\left(\int_{0}^{\infty} \mu(s) \Delta^{2} \eta^{t}(s) d s\right) \theta(t) d t, \omega\right\rangle-\left\langle\int_{0}^{T} \Delta u_{t}(t) \theta(t) d t, \omega\right\rangle \\
+\left\langle\int_{0}^{T} f(u(t)) \theta(t) d t, \omega\right\rangle=\left\langle\int_{0}^{T} h(t) \theta(t) d t, \omega\right\rangle, \\
-\left\langle\int_{0}^{T} \eta^{t} \vartheta^{\prime}(t) d t, \xi\right\rangle_{\mu}=-\left\langle\int_{0}^{T} \eta_{s}^{t} \vartheta(t) d t, \xi\right\rangle_{\mu}-\left\langle\int_{0}^{T} u(t) \vartheta^{\prime}(t) d t, \xi\right\rangle_{\mu},
\end{gathered}
$$

ou ainda,

$$
\begin{gathered}
\left\langle\int_{0}^{T} u_{t t}(t) \theta(t) d t, \omega\right\rangle+\left\langle\int_{0}^{T} \Delta^{2} u(t) \theta(t) d t, \omega\right\rangle-\left\langle\int_{0}^{T} \Delta_{p} u(t) \theta(t) d t, \omega\right\rangle \\
+\left\langle\int_{0}^{T}\left(\int_{0}^{\infty} \mu(s) \Delta^{2} \eta^{t}(s) d s\right) \theta(t) d t, \omega\right\rangle-\left\langle\int_{0}^{T} \Delta u_{t}(t) \theta(t) d t, \omega\right\rangle \\
+\left\langle\int_{0}^{T} f(u(t)) \theta(t) d t, \omega\right\rangle=\left\langle\int_{0}^{T} h(t) \theta(t) d t, \omega\right\rangle, \\
\left\langle\int_{0}^{T} \eta_{t}^{t} \vartheta(t) d t, \xi\right\rangle_{\mu}=-\left\langle\int_{0}^{T} \eta_{s}^{t} \vartheta(t) d t, \xi\right\rangle_{\mu}+\left\langle\int_{0}^{T} u_{t}(t) \vartheta(t) d t, \xi\right\rangle_{\mu},
\end{gathered}
$$

para todo par $(\omega, \xi) \in V_{2} \times \mathcal{M}_{2}$, onde agora a notações

$$
\langle\cdot, \cdot\rangle \text { designa a dualidade }\langle\cdot, \cdot\rangle_{V_{2}^{\prime}, V_{2}} \text { e }\langle\cdot, \cdot\rangle_{\mu} \text { designa a dualidade }\langle\cdot, \cdot\rangle_{\mathcal{M}_{2}^{\prime}, \mathcal{M}_{2}} \text {. }
$$

Isto nos leva a concluir que o par $(u, \eta)$ é uma solução de

$$
\begin{aligned}
& u_{t t}+\Delta^{2} u-\Delta_{p} u+\int_{0}^{\infty} \mu(s) \Delta^{2} \eta^{t}(s) d s-\Delta u_{t}+f(u)=h \\
& \eta_{t}=-\eta_{s}+u_{t}
\end{aligned}
$$

em $\mathcal{D}^{\prime}\left(0, T ; V_{2}^{\prime}\right) \times \mathcal{D}^{\prime}\left(0, T ; \mathcal{M}_{2}^{\prime}\right)$.

Agora notemos que de (3.70) e (3.77) obtemos

$$
\begin{aligned}
& \Delta^{2} u, \int_{0}^{\infty} \mu(s) \Delta^{2} \eta^{t}(s) d s \in L^{\infty}\left(0, T ; V_{2}^{\prime}\right), \\
& \Delta u_{t} \in L^{2}\left(0, T ; V_{1}^{\prime}\right) \hookrightarrow L^{2}\left(0, T ; V_{2}^{\prime}\right), \\
& h \in L^{2}\left(0, T ; V_{0}\right) \hookrightarrow L^{2}\left(0, T ; V_{2}^{\prime}\right) .
\end{aligned}
$$


Além disso, é simples verificar que

$$
f(u), \Delta_{p} u \in L^{\infty}\left(0, T ; V_{2}^{\prime}\right) .
$$

Com efeito, em primeiro lugar note que das hipóteses (3.10)-(3.12), temos

$$
\begin{aligned}
\|f(u(t))\|_{2}^{2} & =\int_{\Omega}|f(u(t))|^{2} d x \\
& \leq 2 k_{0} \int_{\Omega}\left(|u(t)|^{2}+|u(t)|^{2(\rho+1)}\right) d x \\
& =2 k_{0}\left(\|u(t)\|_{2}^{2}+\|u(t)\|_{2(\rho+1)}^{2(\rho+1)}\right) \\
& \leq C_{\rho}\left(\|\Delta u(t)\|_{2}^{2}+\|\Delta u(t)\|_{2}^{2(\rho+1)}\right),
\end{aligned}
$$

onde na última desigualdade usamos a inclusão $V_{2} \hookrightarrow L^{2(\rho+1)}$. Logo, combinando (3.70) e (3.77) vem que

$$
f(u) \in L^{\infty}\left(0, T ; V_{0}\right) \hookrightarrow L^{\infty}\left(0, T ; V_{2}^{\prime}\right) .
$$

Por outro lado, para $\phi \in V_{2} \hookrightarrow W_{0}^{1,2(p-1)}(\Omega)$, a desigualdade de Hölder implica que

$$
\begin{aligned}
\left|\left\langle\Delta_{p} u(t), \phi\right\rangle\right| & =\left|\left\langle|\nabla u(t)|^{p-2} \nabla u(t), \nabla \phi\right\rangle\right| \\
& \leq \int_{\Omega}|\nabla u(t)|^{p-1}|\nabla \phi| d x \\
& \leq\left[\int_{\Omega}|\nabla u(t)|^{2(p-1)} d x\right]^{1 / 2}\left[\int_{\Omega}|\nabla \phi|^{2} d x\right]^{1 / 2} \\
& =\|\nabla u(t)\|_{2(p-1)}^{p-1}\|\nabla \phi\|_{2} \\
& \leq C_{p}\|\Delta u(t)\|_{2}^{p-1}\|\Delta \phi\|_{2},
\end{aligned}
$$

de onde vem que

$$
\left\|\Delta_{p} u(t)\right\|_{V_{2}^{\prime}} \leq C_{p}\|\Delta u(t)\|_{2}^{p-1},
$$

e novamente de (3.70) obtemos (3.80).

Sendo assim, combinando a primeira equação em (3.78) com (3.79)-(3.80), concluimos que

$$
u_{t t}=-\Delta^{2} u+\Delta_{p} u-\int_{0}^{\infty} \mu(s) \Delta^{2} \eta^{t}(s) d s+\Delta u_{t}-f(u)+h \in L^{2}\left(0, T ; V_{2}^{\prime}\right),
$$

o que mostra (3.75) e, como consequência, implica que a igualdade em (3.78) 1 vale em $L^{2}\left(0, T ; V_{2}^{\prime}\right)$.

Resta verificar (3.76). Contudo, este fato é uma consequência imediata de $(3.70)_{2}$ e de $(3.78)_{2}$.

Isto encerra a prova do item $(i)$ do Teorema 3.3.1.

\subsubsection{Estimativa a priori 2}

Aproveitando a notação das subseções anteriores, determinaremos a seguir que para dados iniciais um pouco mais regulares, obteremos também soluções fracas para o problema (3.5)-(3.8) com mais 
regularidade, conforme exposto em (3.19). Iniciaremos por estabelecer uma estimativa, digamos, mais regular para as soluções do problema aproximado.

Consideremos agora os dados iniciais $\left(u_{0}, u_{1}, \eta_{0}\right) \in \mathcal{H}_{1}$ e, como anteriormente, sejam $u^{m}(t)$ e $\eta^{t, m}$ soluções do problema aproximado (3.22)-(3.23) com condições iniciais (3.24), onde agora

$$
u_{0}^{m} \rightarrow u_{0} \quad \text { em } \quad V_{3}, \quad u_{1}^{m} \rightarrow u_{1} \quad \text { em } \quad V_{1} \quad \text { e } \quad \eta_{0}^{m} \rightarrow \eta_{0} \quad \text { em } \quad \mathcal{M}_{3} .
$$

Multiplicando as equações (3.22) por $y_{m j}^{\prime}(t) \lambda_{j}^{1 / 2}$ e (3.23) por $g_{m j}(t) \lambda_{j}^{1 / 2}$ e somando de $j=$ $1, \ldots, m$, então

$$
\begin{aligned}
& -\left(u_{t t}^{m}(t), \Delta u_{t}^{m}(t)\right)-\left(\Delta u^{m}(t), \Delta\left(\Delta u_{t}^{m}(t)\right)\right)+\left\langle\Delta_{p}\left(u^{m}(t)\right), \Delta u_{t}^{m}(t)\right\rangle \\
& \quad-\left(\eta^{t, m}, \Delta u_{t}^{m}(t)\right)_{\mu, 2}+\left\|\Delta u_{t}^{m}(t)\right\|_{2}^{2}-\left(f\left(u^{m}(t)\right), \Delta u_{t}^{m}(t)\right)=-\left(h(t), \Delta u_{t}^{m}(t)\right), \\
& -\left(\partial_{t} \eta^{t, m}, \Delta \eta^{t, m}\right)_{\mu, 2}=\left(\partial_{s} \eta^{t, m}, \Delta \eta^{t, m}\right)_{\mu, 2}-\left(u_{t}^{m}(t), \Delta \eta^{t, m}\right)_{\mu, 2},
\end{aligned}
$$

onde, devido as condições fronteira em (3.21), temos $-\Delta \omega_{j}=\lambda_{j}^{1 / 2} \omega_{j}$ para $j \in \mathbb{N}$. Levando em consideração, novamente, que a base de Galerkin $\left(\omega_{j}\right)_{j \in \mathbb{N}}$ satisfaz as condições de fronteira do problema (3.21), então a fórmula de Green implica que

$$
\begin{gathered}
-\left(u_{t t}^{m}(t), \Delta u_{t}^{m}(t)\right)=\left(\nabla u_{t t}^{m}(t), \nabla u_{t}^{m}(t)\right), \\
-\left(\Delta u^{m}(t), \Delta\left(\Delta u_{t}^{m}(t)\right)\right)=\left(\nabla \Delta u^{m}(t), \nabla \Delta u_{t}^{m}(t)\right)
\end{gathered}
$$

$\mathrm{e}$

$$
\begin{aligned}
-\left(\eta^{t, m}, \Delta u_{t}^{m}(t)\right)_{\mu, 2} & =-\int_{0}^{\infty} \mu(s)\left(\Delta \eta^{t, m}(s), \Delta\left(\Delta u_{t}^{m}(t)\right)\right) d s \\
& =\int_{0}^{\infty} \mu(s)\left(\nabla \Delta \eta^{t, m}(s), \nabla \Delta u_{t}^{m}(t)\right) d s \\
& =\left(\eta^{t, m}, u_{t}^{m}(t)\right)_{\mu, 3} .
\end{aligned}
$$

Além disso, também pela fórmula de Green, temos

$$
\begin{aligned}
-\left(\partial \eta^{t, m}, \Delta \eta^{t, m}\right)_{\mu, 2} & =-\int_{0}^{\infty} \mu(s)\left(\Delta\left(\partial \eta^{t, m}(s)\right), \Delta\left(\Delta \eta^{t, m}(s)\right)\right) d s \\
& =\int_{0}^{\infty} \mu(s)\left(\nabla \Delta \partial \eta^{t, m}(s), \nabla \Delta \eta^{t, m}(s)\right) d s \\
& =\left(\partial \eta^{t, m}, \eta^{t, m}\right)_{\mu, 3} .
\end{aligned}
$$

onde a notação $\partial$ menciona as derivadas $\partial_{t}$ e $\partial_{s}$. Logo, as equações (3.82)-(3.83) podem ser reescritas como

$$
\begin{aligned}
& \left(\nabla u_{t t}^{m}(t), \nabla u_{t}^{m}(t)\right)+\left(\nabla \Delta u^{m}(t), \nabla \Delta u_{t}^{m}(t)\right)+\left\langle\Delta_{p}\left(u^{m}(t)\right), \Delta u_{t}^{m}(t)\right\rangle \\
& \quad+\left(\eta^{t, m}, u_{t}^{m}(t)\right)_{\mu, 3}+\left\|\Delta u_{t}^{m}(t)\right\|_{2}^{2}-\left(f\left(u^{m}(t)\right), \Delta u_{t}^{m}(t)\right)=-\left(h(t), \Delta u_{t}^{m}(t)\right) \\
& \left(\partial_{t} \eta^{t, m}, \eta^{t, m}\right)_{\mu, 3}=-\left(\partial_{s} \eta^{t, m}, \eta^{t, m}\right)_{\mu, 3}+\left(u_{t}^{m}(t), \eta^{t, m}\right)_{\mu, 3}
\end{aligned}
$$


Agora, notando que

$$
\begin{aligned}
\left(\nabla u_{t t}^{m}(t), \nabla u_{t}^{m}(t)\right) & =\frac{1}{2} \frac{d}{d t}\left\|\nabla u_{t}^{m}(t)\right\|_{2}^{2}, \\
\left(\nabla \Delta u^{m}(t), \nabla \Delta u_{t}^{m}(t)\right) & =\frac{1}{2} \frac{d}{d t}\left\|\nabla \Delta u^{m}(t)\right\|_{2}^{2}, \\
\left(\partial_{t} \eta^{t, m}, \eta^{t, m}\right)_{\mu, 3} & =\frac{1}{2} \frac{d}{d t}\left\|\eta^{t, m}\right\|_{\mu, 3}^{2},
\end{aligned}
$$

e substituindo a equação (3.85) em (3.84), vem que

$$
\begin{aligned}
\frac{1}{2} \frac{d}{d t}\left\{\left\|\nabla u_{t}^{m}(t)\right\|_{2}^{2}\right. & \left.+\left\|\nabla \Delta u^{m}(t)\right\|_{2}^{2}+\left\|\eta^{t, m}\right\|_{\mu, 3}^{2}\right\}+\left\langle\Delta_{p}\left(u^{m}(t)\right), \Delta u_{t}^{m}(t)\right\rangle \\
& +\left\|\Delta u_{t}^{m}(t)\right\|_{2}^{2}=-\left(\partial_{s} \eta^{t, m}, \eta^{t, m}\right)_{\mu, 3}+\left(f\left(u^{m}(t)\right)-h(t), \Delta u_{t}^{m}(t)\right) .
\end{aligned}
$$

Como a função $u^{m}(t)$ é bem regular, notamos ainda que

$$
\frac{d}{d t}\left\langle\Delta_{p}\left(u^{m}(t)\right), \Delta u^{m}(t)\right\rangle=\left\langle\Delta_{p}\left(u^{m}(t)\right), \Delta u_{t}^{m}(t)\right\rangle+J_{1},
$$

onde

$$
J_{1}=\int_{\Omega}\left\{(2-p)\left|\nabla u^{m}(t)\right|^{p-4}\left(\nabla u^{m}(t) \cdot \nabla u_{t}^{m}(t)\right) \nabla u^{m}(t)+\left|\nabla u^{m}(t)\right|^{p-2} \nabla u_{t}^{m}(t)\right\} \cdot \nabla \Delta u^{m}(t) d x,
$$

Assim sendo, resulta de (3.86) que

$$
\begin{aligned}
\frac{1}{2} \frac{d}{d t}\left\{\left\|\nabla u_{t}^{m}(t)\right\|_{2}^{2}+\left\|\nabla \Delta u^{m}(t)\right\|_{2}^{2}\right. & +\left\|\eta^{t, m}\right\|_{\mu, 3}^{2} \\
+ & \left.+2\left\langle\Delta_{p} u^{m}(t), \Delta u^{m}(t)\right\rangle\right\} \\
& +\left\|u_{t}^{m}(t)\right\|_{2}^{2}=-\left(\partial_{s} \eta^{t, m}, \eta^{t, m}\right)_{\mu, 3}+J_{1}+J_{2}+J_{3},
\end{aligned}
$$

onde

$$
\begin{aligned}
& J_{2}=\left(f\left(u^{m}(t)\right), \Delta u_{t}^{m}(t)\right)=\int_{\Omega} f\left(u^{m}(t)\right) \Delta u_{t}^{m}(t) d x, \\
& J_{3}=-\left(h(t), \Delta u_{t}^{m}(t)\right)=-\int_{\Omega} h(t) \Delta u_{t}^{m}(t) d x .
\end{aligned}
$$

A seguir vamos exibir estimativas convenientes para os termos do lado direito da igualdade em (3.87). Para simplificar a notação, usaremos o mesmo parâmetro $C$ para denotar diferentes constantes positivas que não dependem de $m$ e $t$.

Em primeiro lugar, devido as condições (3.13)-(3.15) e como $\eta^{t, m}(0)=0$, temos de forma análoga a (3.49) que

$$
-\left(\partial_{s} \eta^{t, m}, \eta^{t, m}\right)_{\mu, 3} \leq-\frac{k_{2}}{2}\left\|\eta^{t, m}\right\|_{\mu, 3}^{2} \leq 0 .
$$

Resta-nos estimar $J_{1}, J_{2}$ e $J_{3}$. As estimativas para $J_{1}$ e $J_{2}$ serão similares às já feitas na subseção "Estimativa a priori 2" do Capítulo 2. Iniciando com $J_{1}$, note que usando a desigualdade de Hölder generalizada, com $\frac{p-2}{2(p-1)}+\frac{1}{2(p-1)}+\frac{1}{2}=1$, a estimativa (3.54) e como $V_{2} \hookrightarrow W_{0}^{1,2(p-1)}(\Omega)$, então

$$
\begin{aligned}
\left|J_{1}\right| & \leq(p-1) \int_{\Omega}\left|\nabla u^{m}(t)\right|^{p-2}\left|\nabla u_{t}^{m}(t)\right|\left|\nabla \Delta u^{m}(t)\right| d x \\
& \leq(p-1)\left\|\nabla u^{m}(t)\right\|_{2(p-1)}^{p-2}\left\|\nabla u_{t}^{m}(t)\right\|_{2(p-1)}\left\|\nabla \Delta u^{m}(t)\right\|_{2} \\
& \leq C_{p}\left\|\Delta u^{m}(t)\right\|_{2}^{p-2}\left\|\Delta u_{t}^{m}(t)\right\|_{2}\left\|\nabla \Delta u^{m}(t)\right\|_{2} \\
& \leq C\left\|\Delta u_{t}^{m}(t)\right\|_{2}\left\|\nabla \Delta u^{m}(t)\right\|_{2},
\end{aligned}
$$


para alguma constante $C>0$. Pela desigualdade de Young $\operatorname{com} \epsilon=1 / 4$, obtemos

$$
\left|J_{1}\right| \leq \frac{1}{4}\left\|\Delta u_{t}^{m}(t)\right\|_{2}^{2}+\frac{C}{2}\left\|\nabla \Delta u^{m}(t)\right\|_{2}^{2}
$$

para alguma constante $C>0$.

Para estimar $J_{2}$, notamos inicialmente que das hipóteses (3.10)-(3.11), das inclusões contínuas $V_{3} \hookrightarrow V_{2} \hookrightarrow L^{2(\rho+1)}(\Omega)$ e da estimativa (3.54), vem que

$$
\begin{aligned}
\left\|f\left(u^{m}(t)\right)\right\|_{2}^{2} & =\int_{\Omega}\left|f\left(u^{m}(t)\right)\right|^{2} d x \\
& \leq 2 k_{0} \int_{\Omega}\left(\left|u^{m}(t)\right|^{2}+\left|u^{m}(t)\right|^{2(\rho+1)}\right) d x \\
& =2 k_{0}\left(\left\|u^{m}(t)\right\|_{2}^{2}+\left\|u^{m}(t)\right\|_{2(\rho+1)}^{2(\rho+1)}\right) \\
& \leq C_{\rho}\left(\left\|\Delta u^{m}(t)\right\|_{2}^{2}+\left\|\Delta u^{m}(t)\right\|_{2}^{2(\rho+1)}\right) \\
& =C_{\rho}\left(1+\left\|\Delta u^{m}(t)\right\|_{2}^{2 \rho}\right)\left\|\Delta u^{m}(t)\right\|_{2}^{2} \\
& \leq \frac{C}{2}\left\|\nabla \Delta u^{m}(t)\right\|_{2}^{2},
\end{aligned}
$$

para alguma constante $C>0$. Logo, novamente aplicando a desigualdade de Young com $\epsilon=1 / 4$, obtemos

$$
\begin{aligned}
\left|J_{2}\right| & \leq\left\|f\left(u^{m}(t)\right)\right\|_{2}\left\|\Delta u_{t}^{m}(t)\right\|_{2} \\
& \leq\left\|f\left(u^{m}(t)\right)\right\|_{2}^{2}+\frac{1}{4}\left\|\Delta u_{t}^{m}(t)\right\|_{2}^{2} \\
& \leq \frac{C}{2}\left\|\nabla \Delta u^{m}(t)\right\|_{2}^{2}+\frac{1}{4}\left\|\Delta u_{t}^{m}(t)\right\|_{2}^{2} .
\end{aligned}
$$

Com relação a $J_{3}$ temos, simplesmente pela desigualdade de Young com $\epsilon=1 / 4$, que

$$
\left|J_{3}\right| \leq\|h(t)\|_{2}\left\|\Delta u_{t}^{m}(t)\right\|_{2} \leq\|h(t)\|_{2}^{2}+\frac{1}{4}\left\|\Delta u_{t}^{m}(t)\right\|_{2}^{2} .
$$

Assim sendo, inserindo as estimativas (3.88)-(3.91) na identidade (3.87), resulta que

$$
\frac{d}{d t} F^{m}(t)+\frac{1}{2}\left\|\Delta u_{t}^{m}(t)\right\|_{2}^{2} \leq 2\|h(t)\|_{2}^{2}+2 C\left\|\nabla \Delta u^{m}(t)\right\|_{2}^{2}
$$

para alguma constante $C>0$, onde denotamos

$$
F^{m}(t)=\left\|\nabla u_{t}^{m}(t)\right\|_{2}^{2}+\left\|\nabla \Delta u^{m}(t)\right\|_{2}^{2}+\left\|\eta^{t, m}\right\|_{\mu, 3}^{2}+2\left\langle\Delta_{p} u^{m}(t), \Delta u^{m}(t)\right\rangle .
$$

Como anteriormente (ver seção 2.3 - estimativa a priori 2), usando a estimativa (3.54), existe uma constante $C>0$ tal que

$$
\left|\left\langle\Delta_{p} u^{m}(t), \Delta u^{m}(t)\right\rangle\right| \leq C+\frac{1}{4}\left\|\nabla \Delta u^{m}(t)\right\|_{2}^{2},
$$

de onde vem que

$$
2\left\langle\Delta_{p} u^{m}(t), \Delta u^{m}(t)\right\rangle \geq-2 C-\frac{1}{2}\left\|\nabla \Delta u^{m}(t)\right\|_{2}^{2}
$$


Combinando isto com a expressão para $F^{m}(t)$, temos

$$
\frac{1}{2}\left\{\left\|\nabla u_{t}^{m}(t)\right\|_{2}^{2}+\left\|\nabla \Delta u^{m}(t)\right\|_{2}^{2}+\left\|\eta^{t, m}\right\|_{\mu, 3}^{2}\right\} \leq F^{m}(t)+2 C .
$$

Por outro lado, integrando (3.92) de 0 a $t \leq T$, segue que

$$
F^{m}(t)+\frac{1}{2} \int_{0}^{t}\left\|\Delta u_{t}^{m}(\tau)\right\|_{2}^{2} d \tau \leq F^{m}(0)+2\|h\|_{L^{2}\left(0, T ; L^{2}(\Omega)\right)}^{2}+2 C \int_{0}^{t}\left\|\nabla \Delta u^{m}(\tau)\right\|_{2}^{2} d \tau
$$

Agora, das estimativas (3.94) e (3.95), vem que

$$
\begin{aligned}
\left\|\nabla u_{t}^{m}(t)\right\|_{2}^{2} & +\left\|\nabla \Delta u^{m}(t)\right\|_{2}^{2}+\left\|\eta^{t, m}\right\|_{\mu, 3}^{2}+\int_{0}^{t}\left\|\Delta u_{t}^{m}(\tau)\right\|_{2}^{2} d \tau \\
& \leq 2 F^{m}(0)+4\|h\|_{L^{2}\left(0, T ; L^{2}(\Omega)\right)}^{2}+4 C+4 C \int_{0}^{t}\left\|\nabla \Delta u^{m}(\tau)\right\|_{2}^{2} d \tau .
\end{aligned}
$$

para alguma constante $C>0$ dependendo apenas dos dados iniciais e independente de $m$ e $t$. Além disso, usando novamente (3.93), notemos que

$$
\begin{aligned}
F^{m}(0) & =\left\|\nabla u_{1}^{m}\right\|_{2}^{2}+\left\|\nabla \Delta u_{0}^{m}\right\|_{2}^{2}+\left\|\eta_{0}^{m}\right\|_{\mu, 3}^{2}+2\left\langle\Delta_{p} u_{0}^{m}, \Delta u_{0}^{m}\right\rangle \\
& \leq\left\|\nabla u_{1}^{m}\right\|_{2}^{2}+\frac{3}{2}\left\|\nabla \Delta u_{0}^{m}\right\|_{2}^{2}+\left\|\eta_{0}^{m}\right\|_{\mu, 3}^{2}+2 C
\end{aligned}
$$

Assim, desta estimativa e devido às convergências (3.81), existe uma constante positiva $C_{1}=$ $C_{1}\left(|| \nabla u_{1}\left\|_{2},\right\| \nabla \Delta u_{0}\left\|_{2},\right\| \eta_{0}\left\|_{\mu, 3},\right\| h \|_{L^{2}\left(0, T ; L^{2}(\Omega)\right)},|\Omega|, T\right)>0$ tal que

$$
2 F^{m}(0)+4\|h\|_{L^{2}\left(0, T ; L^{2}(\Omega)\right)}^{2}+4 C \leq C_{1} .
$$

Logo, a estimativa (3.96) se reduz a

$$
\left\|\nabla u_{t}^{m}(t)\right\|_{2}^{2}+\left\|\nabla \Delta u^{m}(t)\right\|_{2}^{2}+\left\|\eta^{t, m}\right\|_{\mu, 3}^{2}+\int_{0}^{t}\left\|\Delta u_{t}^{m}(\tau)\right\|_{2}^{2} d \tau \leq C_{1}+C_{1} \int_{0}^{t}\left\|\nabla \Delta u^{m}(\tau)\right\|_{2}^{2} d \tau .
$$

Pela desigualdade de Gronwall, concluimos que

$$
\left\|\nabla u_{t}^{m}(t)\right\|_{2}^{2}+\left\|\nabla \Delta u^{m}(t)\right\|_{2}^{2}+\left\|\eta^{t, m}\right\|_{\mu, 3}^{2}+\int_{0}^{t}\left\|\Delta u_{t}^{m}(\tau)\right\|_{2}^{2} d \tau \leq M_{2},
$$

para todos $t \in[0, T]$ e $m \in \mathbb{N}$, onde $M_{2}>0$ é tal que

$$
M_{2}=M_{2}\left(|| \nabla u_{1}\left\|_{2},\right\| \nabla \Delta u_{0}\left\|_{2},\right\| \eta_{0}\left\|_{\mu, 3},\right\| h \|_{L^{2}\left(0, T ; L^{2}(\Omega)\right)},|\Omega|, T\right) .
$$

Em particular, a estimativa (3.97) implica que

$$
\begin{array}{lll}
\left(u^{m}\right) & \text { é limitada em } & L^{\infty}\left(0, T ; V_{3}\right), \\
\left(u_{t}^{m}\right) & \text { é limitada em } & L^{\infty}\left(0, T ; V_{1}\right) \cap L^{2}\left(0, T ; V_{2}\right), \\
\left(\eta^{m}\right) & \text { é limitada em } & L^{\infty}\left(0, T ; \mathcal{M}_{3}\right) .
\end{array}
$$




\subsubsection{Passagem ao limite e solução fraca mais regular}

Em virtude de (3.98) e aplicando os Teoremas 1.2.18 e 1.2.19, podemos extrair subsequências de $\left(u^{m}\right)$ e $\left(\eta^{m}\right)$, que ainda denotaremos por $\left(u^{m}\right)$ e $\left(\eta^{m}\right)$, tais que

$$
\begin{array}{clll}
u^{m} \stackrel{*}{\rightarrow} u & \text { em } & L^{\infty}\left(0, T ; V_{3}\right), \\
u_{t}^{m} \stackrel{*}{\rightarrow} u_{t} & \text { em } & L^{\infty}\left(0, T ; V_{1}\right), \\
u_{t}^{m} \rightarrow u_{t} & \text { em } & L^{2}\left(0, T ; V_{2}\right), \\
\eta^{m} \stackrel{*}{\rightarrow} \eta & \text { em } & L^{\infty}\left(0, T ; \mathcal{M}_{3}\right) .
\end{array}
$$

De posse desses limites em (3.99), em conjunto com as convergências obtidas em (3.56)-(3.58), podemos passar, com maior razão, o limite no problema aproximado e obter uma solução fraca mais regular para o problema (3.5)-(3.8). Mais precisamente, procedendo analogamente como na subseção 3.3.3, concluimos que

$$
\begin{aligned}
& \frac{d}{d t}\left(u_{t}, \omega\right)+(\Delta u, \Delta \omega)-\left\langle\Delta_{p}(u), \omega\right\rangle+(\eta, \omega)_{\mu, 2}-\left(\Delta u_{t}, \omega\right)+(f(u), \omega)=(h, \omega), \\
& \frac{d}{d t}(\eta, \xi)_{\mu, 2}=-\left(\partial_{s} \eta, \xi\right)_{\mu, 2}+\frac{d}{d t}(u, \xi)_{\mu, 2},
\end{aligned}
$$

em $\mathcal{D}^{\prime}(0, T)$, para todo par $(\omega, \xi) \in V_{3} \times \mathcal{M}_{3}$. Isto infere que o limite $z:=\left(u, u_{t}, \eta\right) \in \mathcal{H}_{1}$ satisfaz a (3.16)-(3.17) com

$$
\begin{aligned}
& u \in L^{\infty}\left(0, T ; V_{3}\right), \\
& u_{t} \in L^{\infty}\left(0, T ; V_{1}\right) \cap L^{2}\left(0, T ; V_{2}\right), \\
& \eta \in L^{\infty}\left(0, T ; \mathcal{M}_{3}\right),
\end{aligned}
$$

provando também a condição (3.19). Mais adiante, mostraremos também que $z \in C([0, T], \mathcal{H})$. A condição inicial $z(0)=\left(u_{0}, u_{1}, \eta_{0}\right)$ é imediata, sendo similar à anterior.

Agora, como anteriormente, temos:

Observação 3.3.2. Além do exposto acima, afirmamos também que:

$$
\begin{aligned}
u_{t t} & \in L^{2}\left(0, T ; V_{1}^{\prime}\right), \\
\eta_{t}+\eta_{s} & \in L^{\infty}\left(0, T ; \mathcal{M}_{1}\right) \cap L^{2}\left(0, T ; \mathcal{M}_{2}\right) .
\end{aligned}
$$

Com efeito, usando o Teorema de Representação de Riesz-Fréchet, vale também as seguintes cadeias de inclusões contínuas

$$
V_{3} \hookrightarrow V_{2} \hookrightarrow W_{0}^{1, p} \hookrightarrow V_{1} \hookrightarrow V_{0} \hookrightarrow V_{1}^{\prime} \hookrightarrow W^{-1, p^{\prime}} \hookrightarrow V_{2}^{\prime} \hookrightarrow V_{3}^{\prime} \text { e } \mathcal{M}_{3} \hookrightarrow \mathcal{M}_{0} \hookrightarrow \mathcal{M}_{3}^{\prime} .
$$

Mediante a essas inclusões podemos enxergar as equações acima em uma dualidade (ver subseção 3.3.3), o que nos permite concluir que o par $(u, \eta)$ é uma solução de

$$
\begin{aligned}
& u_{t t}+\Delta^{2} u-\Delta_{p} u+\int_{0}^{\infty} \mu(s) \Delta^{2} \eta^{t}(s) d s-\Delta u_{t}+f(u)=h \\
& \eta_{t}=-\eta_{s}+u_{t}
\end{aligned}
$$


em $\mathcal{D}^{\prime}\left(0, T ; V_{3}^{\prime}\right) \times \mathcal{D}^{\prime}\left(0, T ; M_{3}^{\prime}\right)$. Agora afirmamos que:

$$
\Delta^{2} u, f(u), \Delta_{p} u \in L^{\infty}\left(0, T ; V_{1}^{\prime}\right) .
$$

Com efeito, das hipóteses (3.11)-(3.12) é imediato ver (assim como anteriormente) que

$$
f(u) \in L^{\infty}\left(0, T ; V_{0}\right) \hookrightarrow L^{\infty}\left(0, T ; V_{1}^{\prime}\right)
$$

Além disso, para $\phi \in V_{1}$, obtemos

$$
\left|\left\langle\Delta^{2} u(t), \phi\right\rangle\right|=|(\nabla \Delta u(t), \nabla \phi)| \leq|| \nabla \Delta u(t)||_{2}\|\nabla \phi\|_{2},
$$

de onde tiramos, usando $(3.100)_{1}$, que

$$
\left\|\Delta^{2} u(t)\right\|_{V_{1}^{\prime}} \leq\|\nabla \Delta u(t)\|_{2}<\infty \quad \text { q.s } \quad \text { em } \quad[0, T] .
$$

Mais ainda, como

$$
\left|\left\langle\Delta_{p} u(t), \phi\right\rangle\right|=\left|\left\langle|\nabla u(t)|^{p-2} \nabla u(t), \nabla \phi\right\rangle\right| \leq \int_{\Omega}|\nabla u(t)|^{p-1}|\nabla \phi| d x \leq\left\|\nabla u(t)||_{2(p-1)}^{p-1}|| \nabla \phi\right\|_{2},
$$

então existe uma constante $C_{p}>0$ tal que

$$
\left\|\Delta_{p} u(t)\right\|_{V_{1}^{\prime}} \leq C_{p}\|\Delta u(t)\|_{2}^{p-1}<\infty
$$

onde usamos a estimativa fraca (3.70). Com isto, a condição (3.105) fica satisfeita. O mesmo raciocínio se aplica, usando (3.100) $)_{3}$ e as inclusões (3.103), para mostrar que

$$
\int_{0}^{\infty} \mu(s) \Delta^{2} \eta^{t}(s) d s \in L^{\infty}\left(0, T ; V_{1}^{\prime}\right) .
$$

Também, de (3.100) 2 e da hipótese sobre $h$, segue imediatamente que

$$
\Delta u_{t}, h \in L^{2}\left(0, T ; V_{0}\right) \hookrightarrow L^{2}\left(0, T ; V_{1}^{\prime}\right) .
$$

Logo, combinando a primeira equação em (3.104) com (3.105)-(3.107), obtemos

$$
u_{t t}=-\Delta^{2} u+\Delta_{p} u-\int_{0}^{\infty} \mu(s) \Delta^{2} \eta^{t}(s) d s+\Delta u_{t}-f(u)+h \in L^{2}\left(0, T ; V_{1}^{\prime}\right) .
$$

Isto mostra (3.101) como desejado e, consequentemente, a igualdade em (3.104) 1 vale em $L^{2}\left(0, T ; V_{1}^{\prime}\right)$, onde relembramos que $V_{1}^{\prime}:=H^{-1}(\Omega)$ é o dual de $H_{0}^{1}(\Omega)$. Por fim, a condição (3.102) é uma consequência imediata de $(3.100)_{2}$ e de $(3.104)_{2}$.

Para concluir este item, mostraremos a continuidade da função $z=\left(u, u_{t}, \eta\right)($ de $[0, T]$ em $\mathcal{H})$ comentada anteriormente, ou seja, que

$$
z \in C([0, T], \mathcal{H}), \quad T>0 .
$$


De fato, das condições expostas anteriormente (ver (3.70), (3.75)-(3.76), (3.100) e (3.101)-(3.102)) extraimos, de modo conveniente, pelo menos que

$$
\begin{aligned}
u & \in L^{\infty}\left(0, T ; V_{2}\right) \cap C\left([0, T], V_{1}\right), \\
u_{t} & \in L^{\infty}\left(0, T ; V_{0}\right) \cap L^{2}\left(0, T ; V_{1}\right), \\
u_{t t} & \in L^{2}\left(0, T ; V_{2}^{\prime}\right) \\
\eta & \in L^{\infty}\left(0, T ; \mathcal{M}_{2}\right), \\
\eta_{t} & \in L^{2}\left(0, T ; \mathcal{M}_{2}^{\prime}\right) .
\end{aligned}
$$

Isto implica que

$$
\begin{aligned}
u & \in L^{\infty}\left(0, T ; V_{2}\right) \cap C_{w}\left([0, T], V_{0}\right), \\
u_{t} & \in L^{\infty}\left(0, T ; V_{0}\right) \cap C_{w}\left([0, T], V_{2}^{\prime}\right), \\
\eta & \in L^{\infty}\left(0, T ; \mathcal{M}_{2}\right) \cap C_{w}\left([0, T], M_{2}^{\prime}\right) .
\end{aligned}
$$

Como vale as inclusões (3.77), então aplicando o Lema 1.2.15 segue que

$$
\begin{aligned}
u & \in C_{w}\left([0, T], V_{2}\right) \cap C\left([0, T], V_{1}\right), \\
u_{t} & \in C_{w}\left([0, T], V_{0}\right) \cap L^{2}\left(0, T ; V_{1}\right), \\
\eta & \in C_{w}\left([0, T], \mathcal{M}_{2}\right) .
\end{aligned}
$$

Com esta regularidade, multiplicando a primeira e segunda equações em (3.104) por $u_{t}$ e $\eta$, respectivamente, integrando sobre $\Omega$ e somando as expressões resultantes, deduzimos que

$$
\begin{aligned}
& \frac{1}{2} \frac{d}{d t}\left\{\|\Delta u(t)\|_{2}^{2}+\left\|u_{t}(t)\right\|_{2}^{2}+\left\|\eta^{t}\right\|_{\mu, 2}^{2}\right\} \\
& =\left\langle\Delta_{p} u(t), u_{t}(t)\right\rangle-\left(\eta^{t}, \eta_{s}^{t}\right)_{\mu, 2}-\left\|\nabla u_{t}(t)\right\|_{2}^{2}-\left(f(u(t))-h(t), u_{t}(t)\right),
\end{aligned}
$$

onde os cálculos foram feitos de modo análogo aos anteriores. Agora consideremos $t_{0} \in[0, T)$ qualquer e integrando (3.111) sobre $\left[t_{0}, t\right] \subset[0, T]$ segue que

$$
\begin{aligned}
& \frac{1}{2}\left[\left\{\|\Delta u(t)\|_{2}^{2}+\left\|u_{t}(t)\right\|_{2}^{2}+\left\|\eta^{t}\right\|_{\mu, 2}^{2}\right\}-\left\{\left\|\Delta u\left(t_{0}\right)\right\|_{2}^{2}+\left\|u_{t}\left(t_{0}\right)\right\|_{2}^{2}+\left\|\eta^{t_{0}}\right\|_{\mu, 2}^{2}\right\}\right] \\
& \quad=\int_{t_{0}}^{t}\left\{\left\langle\Delta_{p} u(\tau), u_{t}(\tau)\right\rangle-\left(\eta^{\tau}, \eta_{s}^{\tau}\right)_{\mu, 2}-\left\|\nabla u_{t}(\tau)\right\|_{2}^{2}-\left(f(u(\tau))-h(\tau), u_{t}(\tau)\right)\right\} d \tau .
\end{aligned}
$$

A seguir mostraremos que a integral sobre $\left[t_{0}, t\right]$ do lado direito da identidade (3.112) faz sentido e, além disso, tende a 0 quando $t \rightarrow t_{0}^{+}$. De fato, como $u_{t}(t) \in V_{1}$ e vale a inclusão $V_{2} \hookrightarrow W_{0}^{1,2(p-1)}(\Omega)$, então pela desigualdade de Hölder temos

$$
\begin{aligned}
\left|\left\langle\Delta_{p} u(t), u_{t}(t)\right\rangle\right| & =\left|\left\langle|\nabla u(t)|^{p-2} \nabla u(t), \nabla u_{t}(t)\right\rangle\right| \\
& \leq \int_{\Omega}|\nabla u(t)|^{p-1}\left|\nabla u_{t}(t)\right| d x \\
& \leq\left[\int_{\Omega}|\nabla u(t)|^{2(p-1)} d x\right]^{\frac{1}{2}}\left[\int_{\Omega}\left|\nabla u_{t}(t)\right|^{2} d x\right]^{\frac{1}{2}} \\
& =\left\|\left.\nabla u(t)\right|_{2(p-1)} ^{p-1}\right\| \nabla u_{t}(t) \|_{2} \\
& \leq C_{p}\|\Delta u(t)\|_{2}^{p-1}|| \nabla u_{t}(t) \|_{2},
\end{aligned}
$$


para alguma constante $C_{p}>0$. Além disso, como já vimos anteriormente, usando as hipóteses (3.10)-(3.12) temos

$$
\begin{aligned}
\left|\left(f(u(t)), u_{t}(t)\right)\right| & \leq\|f(u(t))\|_{2}\left\|u_{t}(t)\right\|_{2} \\
& \leq C_{\rho}\left(\|\Delta u(t)\|_{2}+\|\Delta u(t)\|_{2}^{(\rho+1)}\right)\left\|u_{t}(t)\right\|_{2},
\end{aligned}
$$

para alguma constante $C_{\rho}>0$, e também,

$$
\left|\left(h(t), u_{t}(t)\right)\right| \leq\|h(t)\|_{2}\left\|u_{t}(t)\right\|_{2} .
$$

Mais ainda, das condições (3.13)-(3.15) e como $\eta \in L^{\infty}\left(0, T ; \mathcal{M}_{2}\right) \operatorname{com} \eta^{t}(0)=0$, vem que

$$
\begin{aligned}
\left(\eta^{t}, \eta_{s}^{t}\right)_{\mu, 2} & =\int_{0}^{\infty} \mu(s)\left(\Delta \eta^{t}(s), \Delta \eta_{s}^{t}(s)\right) d s \\
& =\frac{1}{2} \underbrace{\int_{0}^{\infty} \frac{d}{d s}\left[\mu(s)\left\|\Delta \eta^{t}(s)\right\|_{2}^{2}\right] d s}_{=0}-\frac{1}{2} \int_{0}^{\infty} \mu^{\prime}(s)\left\|\Delta \eta^{t}(s)\right\|_{2}^{2} d s \\
& =-\frac{1}{2} \int_{0}^{\infty} \mu^{\prime}(s)\left\|\Delta \eta^{t}(s)\right\|_{2}^{2} d s \\
& \geq \frac{k_{2}}{2} \int_{0}^{\infty} \mu(s)\left\|\Delta \eta^{t}(s)\right\|_{2}^{2} d s \\
& =\frac{k_{2}}{2}\left\|\eta^{t}\right\|_{\mu, 2}^{2} .
\end{aligned}
$$

Essas estimativas em conjunto com (3.109) asseguram que a função

$$
\Lambda(\tau)=\left\langle\Delta_{p} u(\tau), u_{t}(\tau)\right\rangle-\left(\eta^{\tau}, \eta_{s}^{\tau}\right)_{\mu, 2}-\left\|\nabla u_{t}(\tau)\right\|_{2}^{2}-\left(f(u(\tau))-h(\tau), u_{t}(\tau)\right)
$$

é integrável sobre $\left[t_{0}, t\right]$ e que $\int_{t_{0}}^{t} \Lambda(\tau) d \tau \rightarrow 0$ quando $t \rightarrow t_{0}^{+}$. Logo, resulta de (3.112) que

$$
\|\Delta u(t)\|_{2}^{2}+\left\|u_{t}(t)\right\|_{2}^{2}+\left\|\eta^{t}\right\|_{\mu, 2}^{2} \stackrel{t \rightarrow t_{0}^{+}}{\longrightarrow}\left\|\Delta u\left(t_{0}\right)\right\|_{2}^{2}+\left\|u_{t}\left(t_{0}\right)\right\|_{2}^{2}+\left\|\eta^{t_{0}}\right\|_{\mu, 2}^{2} .
$$

Portanto, combinando (3.110) com (3.113), concluimos que

$$
\left(u(t), u_{t}(t), \eta^{t}\right) \stackrel{t \rightarrow t_{0}^{+}}{\longrightarrow}\left(u\left(t_{0}\right), u_{t}\left(t_{0}\right), \eta^{t_{0}}\right) \quad \text { em } \quad \mathcal{H} .
$$

Isto mostra a continuidade à direita de $z(t)=\left(u(t), u_{t}(t), \eta^{t}\right)$ em $t_{0} \in[0, T)$. De modo semelhante se motra a continuidade à esquerda de $z(t)$ em $t_{0} \in(0, T]$. Portanto, a condição (3.108) fica assegurada para soluções fracas mais regulares, como queriamos.

Observação 3.3.3. Usando um procedimento de regularização que consiste em aproximar a função $u_{t}$ por uma sequência regular a valores em $V_{2}$, segue também a continuidade (3.108) para soluções fracas. Este procedimento pode ser feito de modo semelhante aos encontrados em Dautray \& Lions [33, Capítulo XVIII, Lema 7], ver também em Ghidaglia \& Temam [46], ou ainda, em Lions \& Magenes [67, Capítulo 3, Lema 8.3], que consiste em usar as mesmas estimativas anteriores com sequências regularizantes.

Portanto, quando o dados iniciais $\left(u_{0}, u_{1}, \eta_{0}\right) \in \mathcal{H}_{1}$, a solução fraca $z:=\left(u, u_{t}, \eta\right)$ do problema (3.5)-(3.8) possui mais regularidade, como exposto em (3.100).

Isto encerra a prova do item ( $i i)$ do Teorema 3.3.1. 


\subsubsection{Dependência contínua e unicidade}

Iniciaremos considerando o caso de soluções mais fortes. Sejam $z_{1}=\left(u, u_{t}, \eta\right)$ e $z_{2}=\left(\widetilde{u}, \widetilde{u}_{t}, \widetilde{\eta}\right)$ duas soluções fracas mais regulares do problema (3.5)-(3.8) correspondentes aos dados iniciais $z_{1,0}=\left(u_{0}, u_{1}, \eta_{0}\right)$ e $z_{2,0}=\left(\widetilde{u}_{0}, \widetilde{u}_{1}, \widetilde{\eta}_{0}\right)$, respectivamente. Definindo $w:=u-\widetilde{u}$ e $\zeta:=\eta-\widetilde{\eta}$, então a função $z:=\left(w, w_{t}, \zeta\right)=z_{1}-z_{2}$ é uma solução mais regular do seguinte problema

$$
\begin{aligned}
& w_{t t}+\Delta^{2} w-\Delta_{p} u+\Delta_{p} \widetilde{u}+\int_{0}^{\infty} \mu(s) \Delta^{2} \zeta^{t}(s) d s-\Delta w_{t}+f(u)-f(\widetilde{u})=0, \\
& \zeta_{t}=-\zeta_{s}+w_{t}
\end{aligned}
$$

com condições iniciais

$$
z(0)=\left(u_{0}-\widetilde{u}_{0}, u_{1}-\widetilde{u}_{1}, \eta_{0}-\widetilde{\eta}_{0}\right)
$$

Com esta regularidade, multiplicando a equações (3.114) por $w_{t} \mathrm{e}$ (3.115) por $\zeta$, integrando sobre $\Omega$ e somando as expressões resultantes, deduzimos que

$$
\begin{aligned}
& \frac{1}{2} \frac{d}{d t}\left\{\|\Delta w(t)\|_{2}^{2}+\left\|w_{t}(t)\right\|_{2}^{2}+\left\|\zeta^{t}\right\|_{\mu, 2}^{2}\right\}+\left\|\nabla w_{t}(t)\right\|_{2}^{2} \\
&=\left\langle\Delta_{p} u(t)-\Delta_{p} \widetilde{u}(t), w_{t}(t)\right\rangle-\left(f(u(t))-f(\widetilde{u}(t)), w_{t}(t)\right)-\left(\zeta^{t}, \zeta_{s}^{t}\right)_{\mu, 2}
\end{aligned}
$$

A seguir vamos estimar os termos do lado direito da igualdade acima de modo conveniente. Em primeiro lugar, usando o Lema 1.3.1, a desigualdade de Hölder generalizada, a condição (3.9) e que $u, \widetilde{u} \in L^{\infty}\left(0, T ; V_{2}\right) \hookrightarrow L^{\infty}\left(0, T ; W_{0}^{1,2(p-1)}(\Omega)\right)$, então

$$
\begin{aligned}
&\left|\left\langle\Delta_{p} u(t)-\Delta_{p} \widetilde{u}(t), w_{t}(t)\right\rangle\right| \\
&=\left|\left\langle|\nabla u(t)|^{p-2} \nabla u(t)-|\nabla \widetilde{u}(t)|^{p-2} \nabla \widetilde{u}(t), \nabla w_{t}(t)\right\rangle\right| \\
& \leq\left.\int_{\Omega}|| \nabla u(t)\right|^{p-2} \nabla u(t)-|\nabla \widetilde{u}(t)|^{p-2} \nabla \widetilde{u}(t)|| \nabla w_{t}(t) \mid d x \\
& \leq M_{p} \int_{\Omega}\left(|\nabla u(t)|^{p-2}+|\nabla \widetilde{u}(t)|^{p-2}\right)|\nabla w(t)|\left|\nabla w_{t}(t)\right| d x \\
& \leq M_{p}\left(\|\nabla u(t)\|_{2(p-1)}^{p-2}+\|\nabla \widetilde{u}(t)\|_{2(p-1)}^{p-2}\right)\|\nabla w(t)\|_{2(p-1)}\left\|\nabla w_{t}(t)\right\|_{2} \\
& \leq C_{p}\|\Delta w(t)\|_{2}\left\|\nabla w_{t}(t)\right\|_{2},
\end{aligned}
$$

para alguma constante $C_{p}>0$. Logo, pela desigualdade de Young, obtemos

$$
\left|\left\langle\Delta_{p} u(t)-\Delta_{p} \widetilde{u}(t), w_{t}(t)\right\rangle\right| \leq C\|\Delta w(t)\|_{2}^{2}+\frac{1}{2}\left\|\nabla w_{t}(t)\right\|_{2}^{2},
$$

para alguma constante $C>0$. 
Agora, das hipóteses (3.10)-(3.11), da desigualdade de Hölder generalizada e como $u, \widetilde{u} \in$ $L^{\infty}\left(0, T ; V_{2}\right) \hookrightarrow L^{\infty}\left(0, T ; L^{2(\rho+1)}(\Omega)\right)$, então

$$
\begin{aligned}
\mid(f(u(t)) & \left.-f(\widetilde{u}(t)), w_{t}(t)\right) \mid \\
& \leq \int_{\Omega}|f(u(t))-f(\widetilde{u}(t))|\left|w_{t}(t)\right| d x \\
& \leq k_{0} \int_{\Omega}\left(1+|u(t)|^{\rho}+|\widetilde{u}(t)|^{\rho}\right)|w(t)|\left|w_{t}(t)\right| d x \\
& \leq k_{0}\left(|\Omega|^{\frac{\rho}{2(\rho+1)}}+\|u(t)\|_{2(\rho+1)}^{\rho}+\|\widetilde{u}(t)\|_{2(\rho+1)}^{\rho}\right)\|w(t)\|_{2(\rho+1)}\left\|w_{t}(t)\right\|_{2} \\
& \leq C_{\rho}\|\Delta w(t)\|_{2}\left\|w_{t}(t)\right\|_{2}
\end{aligned}
$$

para alguma constante $C_{\rho}>0$. Assim, novamente pela desigualdade de Young, obtemos

$$
\left|\left(f(u(t))-f(\widetilde{u}(t)), w_{t}(t)\right)\right| \leq C\|\Delta w(t)\|_{2}^{2}+\frac{1}{2}\left\|w_{t}(t)\right\|_{2}^{2},
$$

para alguma constante $C>0$.

Mais ainda, das hipóteses (3.13)-(3.15) e como $\zeta \in L^{\infty}\left(0, T ; \mathcal{M}_{2}\right) \operatorname{com} \zeta^{t}(0)=0$,vem que

$$
\begin{aligned}
-\left(\zeta^{t}, \zeta_{s}^{t}\right)_{\mu, 2} & =-\int_{0}^{\infty} \mu(s)\left(\Delta \zeta^{t}(s), \Delta \zeta_{s}^{t}(s)\right) d s \\
& =\frac{1}{2} \int_{0}^{\infty} \mu^{\prime}(s)\left\|\Delta \zeta^{t}(s)\right\|_{2}^{2} d s \\
& \leq-\frac{k_{2}}{2} \int_{0}^{\infty} \mu(s)\left\|\Delta \zeta^{t}(s)\right\|_{2}^{2} d s \\
& \leq \frac{k_{2}}{2}\left\|\zeta^{t}\right\|_{\mu, 2}^{2} .
\end{aligned}
$$

Substituindo as estimativas (3.118)-(3.120) na identidade (3.117), obtemos

$$
\begin{aligned}
\frac{d}{d t}\left\{\|\Delta w(t)\|_{2}^{2}+\left\|w_{t}(t)\right\|_{2}^{2}\right. & \left.+\left\|\zeta^{t}\right\|_{\mu, 2}^{2}\right\}+\left\|\nabla w_{t}(t)\right\|_{2}^{2} \\
& \leq 4 C\|\Delta w(t)\|_{2}^{2}+\left\|w_{t}(t)\right\|_{2}^{2}+k_{2}\left\|\zeta^{t}\right\|_{\mu, 2}^{2},
\end{aligned}
$$

o que implica em

$$
\frac{d}{d t}\left\{\|\Delta w(t)\|_{2}^{2}+\left\|w_{t}(t)\right\|_{2}^{2}+\left\|\zeta^{t}\right\|_{\mu, 2}^{2}\right\} \leq c^{2}\left\{\|\Delta w(t)\|_{2}^{2}+\left\|w_{t}(t)\right\|_{2}^{2}+\left\|\zeta^{t}\right\|_{\mu, 2}^{2}\right\},
$$

para alguma constante $c>0$. Lembrando que $\left(w, w_{t}, \zeta\right)=z_{1}-z_{2}$, então podemos reescrever (3.121) como

$$
\frac{d}{d t}\left\|z_{1}(t)-z_{2}(t)\right\|_{\mathcal{H}}^{2} \leq c^{2}\left\|z_{1}(t)-z_{2}(t)\right\|_{\mathcal{H}}^{2} .
$$

Aplicando a desigualdade de Gronwall em (3.122), obtemos

$$
\left\|z_{1}(t)-z_{2}(t)\right\|_{\mathcal{H}} \leq e^{c t}\left\|z_{1,0}-z_{2,0}\right\|_{\mathcal{H}}, \quad t \in[0, T],
$$

para alguma constante $c>0$. Isto mostra a condição (3.20) e a depêndencia contínua de soluções com relação aos dados iniciais em $\mathcal{H}$ fica assegurada. Em particular, temos unicidade de soluções mais regulares. 
A mesma conclusão vale para soluções fracas fazendo uso de mollifiers, assim como em Pata \& Zucchi [83] ou Giorgi et al [48]. Uma outra abordagem, baseada em argumentos de densidade, segue em Cavalcanti et al [19].

Isto encerra a prova do item $($ iii $)$ e, portanto, a prova do Teorema 3.3.1 está completa.

Observação 3.3.4. Nas seções seguintes vamos avaliar o comportamente assintótico das soluções do problema (3.5)-(3.8) que determinamos nesta seção por meio do Teorema 3.3.1. Para tal propósito, usaremos um procedimento baseado no método de perturbação de energia, ver por exemplo $[19,56]$. Contudo, devido a nova variável $\eta$ nossos argumentos com respeito ao termo de memória (com história) é diferente da usada no Capítulo 2, onde fizemos uso do Lema 2.2.1. Ao invés disso, usaremos somente as hipóteses atribuidas sobre o núcleo da memória $\mu$. Além disso, vale a pena notar que os métodos empregados a seguir claramente serão válidos para soluções fracas mais regulares dadas pelo Teorema 3.3.1 e que, posteriormente, por densidade vale os mesmos procedimentos e afirmações para soluções fracas.

\subsection{O sistema dinâmico}

Com o intuito de lidar com um $C_{0}$-semigrupo no espaço de fase $\mathcal{H}$ a partir da solução do problema (3.5)-(3.8), consideraremos a partir deste momento, e em todo o restante deste trabalho, que a função $h$ é independente do tempo, ou seja, que

$$
h(x, t) \equiv h(x) \in L^{2}(\Omega) \quad \text { para todo } \quad t \geq 0 .
$$

Agora definamos, para cada $t \geq 0$, a família de operadores não necessariamente lineares $S(t): \mathcal{H} \rightarrow \mathcal{H}$ dada por

$$
S(t)\left(u_{0}, u_{1}, \eta_{0}\right)=\left(u(t), u_{t}(t), \eta^{t}\right), \quad t \geq 0,
$$

onde a função $z(t)=\left(u(t), u_{t}(t), \eta^{t}\right)$ é a solução do problema (3.5)-(3.8) dada pelo Teorema 3.3.1 referente aos dados iniciais tomados em $\mathcal{H}$. Devido a existência, unicidade, continuidade e a dependência contínua asseguradas pelo Teorema 3.3.1 é simples verificar que a família $\{S(t)\}_{t \geq 0}$ satisfaz as seguintes condições:

(i) $S(0)=I_{d}: \mathcal{H} \rightarrow \mathcal{H}$ é o operador identidade em $\mathcal{H}$.

(ii) $S(t+\tau)=S(t) S(\tau)$ para todos $t, \tau \geq 0$.

(iii) A aplicação $[0, \infty) \times \mathcal{H} \ni\left(t, z_{0}\right) \longrightarrow S(t)\left(z_{0}\right)=\left(u(t), u_{t}(t), \eta^{t}\right) \in \mathcal{H}$ é contínua para cada $z_{0}=\left(u_{0}, u_{1}, \eta_{0}\right)$ dado.

Além disso, em virtude da condição (3.20) vemos que $S(t)$ é localmente Lipschitz contínuo no espaço de fase $\mathcal{H}$. Assim sendo, mediante à teoria geral revisada na seção 1.6 do Capítulo 1 , 
concluimos que $S(t)$ compõe um $C_{0}$-semigrupo não linear em $\mathcal{H}$ e, também, o par $(\mathcal{H}, S(t))$ é chamado de Sistema Dinâmico.

Portanto, para avaliar o comportamento das soluções do problema (3.5)-(3.8) ao longo do tempo, vamos estabelecer por meio da teoria geral para semigrupos e atratores (conforme exposto na seção 1.6) a evolução ao longo do tempo do $C_{0}$-semigrupo $S(t)$ associado ao problema (3.5)-(3.8) como definido em (3.123). Mais precisamente, nosso objetivo é mostrar a existência de um Atrator Global em $\mathcal{H}$ para $S(t)$, cuja dimensão fractal é finita. Iniciaremos com a noção de conjunto absorvente.

\subsubsection{Existência de conjunto absorvente}

Nesta seção vamos mostrar que o sistema dinâmico $(\mathcal{H}, S(t))$ é dissipativo, isto é, que o $C_{0^{-}}$ semigrupo $S(t)$ possui um conjunto absorvente limitado em $\mathcal{H}$, segundo a Definição 1.6.4.

A existência de um conjunto absorvente limitado para $S(t)$ é assegurada pelo resultado a seguir.

Proposição 3.4.1. Sob as hipóteses do Teorema 3.3.1, o semigrupo $S(t)$ associado ao problema (3.5)-(3.8) possui um conjunto absorvente limitado $\mathcal{B} \subset \mathcal{H}$. Em outras palavras, o sistema dinâmico $(\mathcal{H}, S(t))$ é dissipativo.

Prova. Seja $z=\left(u, u_{t}, \eta\right)$ uma solução do problema (3.5)-(3.8) dada pelo Teorema 3.3.1. O funcional energia correspondente ao sistema (3.5)-(3.8) é dado por

$$
E(t)=\frac{1}{2}\left\|u_{t}(t)\right\|_{2}^{2}+\frac{1}{2}\|\Delta u(t)\|_{2}^{2}+\frac{1}{p}\|\nabla u(t)\|_{p}^{p}+\frac{1}{2}\left\|\eta^{t}\right\|_{\mu, 2}^{2}+\int_{\Omega}[\hat{f}(u(t))-h u(t)] d x .
$$

Em primeiro lugar, notamos que da hipótese (3.12),

$$
\int_{\Omega} \hat{f}(u(t)) d x \geq-k_{1}|\Omega| .
$$

Além disso, como $\lambda_{1}\|u\|_{2}^{2} \leq\|\Delta u\|_{2}^{2}$, onde $\lambda_{1}>0$ denota o primeiro autovalor de $\Delta^{2} \omega=\lambda \omega \operatorname{em} \Omega$ $\operatorname{com} \omega=\Delta \omega=0$ sobre $\Gamma$, então usando a desigualdade de Young com $\epsilon=\lambda_{1} / 4$,

$$
\begin{aligned}
-\int_{\Omega} h u(t) d x & \geq-\frac{\lambda_{1}}{4}\|u(t)\|_{2}^{2}-\frac{1}{\lambda_{1}}\|h\|_{2}^{2} \\
& \geq-\frac{1}{4}\|\Delta u(t)\|_{2}^{2}-\frac{1}{\lambda_{1}}\|h\|_{2}^{2} .
\end{aligned}
$$

Assim,

$$
\int_{\Omega}[\hat{f}(u(t))-h u(t)] d x \geq-\frac{1}{4}\|\Delta u(t)\|_{2}^{2}-\frac{1}{\lambda_{1}}\|h\|_{2}^{2}-k_{1}|\Omega| .
$$

Combinando (3.124) com (3.125), vem que

$$
\frac{1}{4}\left\{\|\Delta u(t)\|_{2}^{2}+\left\|u_{t}(t)\right\|_{2}^{2}+\left\|\eta^{t}\right\|_{\mu, 2}^{2}\right\} \leq E(t)+\frac{1}{\lambda_{1}}\|h\|_{2}^{2}+k_{1}|\Omega|,
$$

ou ainda,

$$
\frac{1}{4}\left\|\left(u(t), u_{t}(t), \eta^{t}\right)\right\|_{\mathcal{H}}^{2} \leq E(t)+\frac{1}{\lambda_{1}}\|h\|_{2}^{2}+k_{1}|\Omega| .
$$


Por outro lado, devido às observações anteriores, procederemos formalmente multiplicando as equações (3.5) por $u_{t}$, (3.6) por $\eta$, integrando sobre $\Omega$ e somando as expressões resultantes, deduzimos que

$$
\frac{d}{d t} E(t)=-\left\|\nabla u_{t}(t)\right\|_{2}^{2}-\left(\eta^{t}, \eta_{s}^{t}\right)_{\mu, 2}
$$

Das condições (3.13)-(3.15) e como $\eta \in L^{\infty}\left(0, T ; \mathcal{M}_{2}\right) \operatorname{com} \eta^{t}(0)=0$, segue que

$$
\begin{aligned}
\left(\eta^{t}, \eta_{s}^{t}\right)_{\mu, 2} & =\int_{0}^{\infty} \mu(s)\left(\Delta \eta^{t}(s), \Delta \eta_{s}^{t}(s)\right) d s \\
& =\frac{1}{2} \underbrace{\int_{0}^{\infty}\left[\mu(s)\left\|\Delta \eta^{t}(s)\right\|_{2}^{2}\right]^{\prime} d s}_{=0}-\frac{1}{2} \int_{0}^{\infty} \mu^{\prime}(s)\left\|\Delta \eta^{t}(s)\right\|_{2}^{2} d s \\
& =-\frac{1}{2} \int_{0}^{\infty} \mu^{\prime}(s)\left\|\Delta \eta^{t}(s)\right\|_{2}^{2} d s \\
& \geq \frac{k_{2}}{2} \int_{0}^{\infty} \mu(s)\left\|\Delta \eta^{t}(s)\right\|_{2}^{2} d s
\end{aligned}
$$

onde aqui a notação ' designa a derivada $d / d s$. Assim,

$$
-\left(\eta^{t}, \eta_{s}^{t}\right)_{\mu, 2} \leq-\frac{k_{2}}{2}\left\|\eta^{t}\right\|_{\mu, 2}^{2}
$$

Substituindo (3.128) em (3.127) obtemos

$$
\frac{d}{d t} E(t) \leq-\left\|\nabla u_{t}(t)\right\|_{2}^{2}-\frac{k_{2}}{2}\left\|\eta^{t}\right\|_{\mu, 2}^{2},
$$

de onde segue que a energia $E(t)$ é decrescente.

Agora, definamos a energia perturbada

$$
E_{\varepsilon}(t)=E(t)+\varepsilon \Psi(t), \quad \varepsilon>0,
$$

onde

$$
\Psi(t)=\int_{\Omega} u_{t}(t) u(t) d x
$$

Os seguintes lemas serão cruciais para concluir a existência de um conjunto absorvente.

Lema 3.4.2. Existe uma constante $C_{1}>0$ tal que

$$
\left|E_{\varepsilon}(t)-E(t)\right| \leq \varepsilon C_{1}\left(E(t)+\frac{1}{\lambda_{1}}\|h\|_{2}^{2}+k_{1}|\Omega|\right), \quad \forall t \geq 0, \forall \varepsilon>0 .
$$

Prova. Usando a desigualdade de Young e a estimativa (3.126), obtemos

$$
\begin{aligned}
|\Psi(t)| & \leq \frac{1}{2}\left\|u_{t}(t)\right\|_{2}^{2}+\frac{1}{2 \lambda_{1}}\|\Delta u(t)\|_{2}^{2} \\
& \leq 2 \max \left\{1,1 / \lambda_{1},\right\}\left(\frac{1}{4}\left\|u_{t}(t)\right\|_{2}^{2}+\frac{1}{4}\|\Delta u(t)\|_{2}^{2}\right) \\
& \leq 2 \max \left\{1,1 / \lambda_{1},\right\}\left(E(t)+\frac{1}{\lambda_{1}}\|h\|_{2}^{2}+k_{1}|\Omega|\right) .
\end{aligned}
$$


Tomando $C_{1}=2 \max \left\{1,1 / \lambda_{1}\right\}$, então

$$
\left|E_{\varepsilon}(t)-E(t)\right|=\varepsilon|\Psi(t)| \leq \varepsilon C_{1}\left(E(t)+\frac{1}{\lambda_{1}}|| h \|_{2}^{2}+k_{1}|\Omega|\right),
$$

o que completa a prova do Lema 3.4.2

Lema 3.4.3. Existe uma constante $\varepsilon_{1}>0$ tal que

$$
\frac{d}{d t} E_{\varepsilon}(t) \leq-\varepsilon E(t), \quad \forall t \geq 0, \forall \varepsilon \in\left(0, \varepsilon_{1}\right] .
$$

Prova. Afirmamos que é suficiente mostrar a existência de constantes $C_{2}, C_{3}>0$ tais que

$$
\frac{d}{d t} \Psi(t) \leq-E(t)+C_{2}\left\|\nabla u_{t}(t)\right\|_{2}^{2}+C_{3}\left\|\eta^{t}\right\|_{\mu, 2}^{2} .
$$

De fato, supondo que a desigualdade (3.131) vale e combinando-a com (3.129), concluimos que

$$
\begin{aligned}
\frac{d}{d t} E_{\varepsilon}(t) & =\frac{d}{d t} E(t)+\varepsilon \frac{d}{d t} \Psi(t) \\
& \leq-\left\|\nabla u_{t}(t)\right\|_{2}^{2}-\frac{k_{2}}{2}\left\|\eta^{t}\right\|_{\mu, 2}^{2}-\varepsilon E(t)+\varepsilon C_{2}\left\|\nabla u_{t}(t)\right\|_{2}^{2}+\varepsilon C_{3}\left\|\eta^{t}\right\|_{\mu, 2}^{2} \\
& =-\varepsilon E(t)-\left(1-\varepsilon C_{2}\right)\left\|\nabla u_{t}(t)\right\|_{2}^{2}-\left(\frac{k_{2}}{2}-\varepsilon C_{3}\right)\left\|\eta^{t}\right\|_{\mu, 2}^{2} \\
& \leq-\varepsilon E(t),
\end{aligned}
$$

onde a última desigualdade é válida para todo $\varepsilon \in\left(0, \varepsilon_{1}\right], \operatorname{com} \varepsilon_{1}=\min \left\{\frac{1}{C_{2}}, \frac{k_{2}}{2 C_{3}}\right\}>0$. Isto comprovaria o desejado. Logo, resta mostrar que (3.131) é verdadeira.

Com efeito, derivando a função em (3.130), usando a equação (3.5) no sentido fraco (uma vez que $u_{t t}$ faz sentido no dual), adicionando e subtraindo $E(t)$ na expressão resultante, obtemos

$$
\begin{aligned}
\frac{d}{d t} \Psi(t)= & -E(t)+\frac{3}{2}\left\|u_{t}(t)\right\|_{2}^{2}-\frac{1}{2}\|\Delta u(t)\|_{2}^{2}-\left(1-\frac{1}{p}\right)\|\nabla u(t)\|_{p}^{p} \\
& +\frac{1}{2}\left\|\eta^{t}\right\|_{\mu, 2}^{2}+I_{1}+I_{2}+I_{3},
\end{aligned}
$$

onde denotamos

$$
\begin{aligned}
I_{1} & :=\int_{\Omega}[\hat{f}(u(t))-f(u(t)) u(t)] d x, \\
I_{2} & :=-\int_{0}^{\infty} \mu(s)\left(\Delta \eta^{t}(s), \Delta u(t)\right) d s, \\
I_{3} & :=-\int_{\Omega} \nabla u(t) \cdot \nabla u_{t}(t) d x .
\end{aligned}
$$

A seguir vamos estimar $I_{1}, I_{2}$ e $I_{3}$. Da hipótese (3.12) segue diretamente que

$$
I_{1} \leq 0
$$

restando estimar apenas $I_{2}$ e $I_{3}$. 
Usando a desigualdade de Young $\operatorname{com} \sigma>0$, obtemos as seguintes estimativas:

$$
\begin{aligned}
\left|I_{2}\right| & \leq \int_{0}^{\infty} \mu(s)\left(\frac{1}{4 \sigma}\left\|\Delta \eta^{t}(s)\right\|_{2}^{2}+\sigma\|\Delta u(t)\|_{2}^{2}\right) d s \\
& =\sigma\left(\int_{0}^{\infty} \mu(s) d s\right)\|\Delta u(t)\|_{2}^{2}+\frac{1}{4 \sigma} \int_{0}^{\infty} \mu(s)\left\|\Delta \eta^{t}(s)\right\|_{2}^{2} d s \\
& =\sigma \mu_{0}\|\Delta u(t)\|_{2}^{2}+\frac{1}{4 \sigma}\left\|\eta^{t}\right\|_{\mu, 2}^{2}
\end{aligned}
$$

e

$$
\begin{aligned}
\left|I_{3}\right| & \leq \sigma\|\nabla u(t)\|_{2}^{2}+\frac{1}{4 \sigma}\left\|\nabla u_{t}(t)\right\|_{2}^{2} \\
& \leq \sigma d_{1}\|\Delta u(t)\|_{2}^{2}+\frac{1}{4 \sigma}\left\|\nabla u_{t}(t)\right\|_{2}^{2},
\end{aligned}
$$

onde $d_{1}>0$ menciona a constante de imersão $V_{2} \hookrightarrow V_{1}$.

Inserindo estas duas últimas estimativas em (3.132) e notando que $1-1 / p \leq 0$, obtemos

$$
\begin{aligned}
\frac{d}{d t} \Psi(t) \leq & -E(t)+\left(\frac{3}{2} d_{2}+\frac{1}{4 \sigma}\right)\left\|\nabla u_{t}(t)\right\|_{2}^{2}+\left(\frac{1}{2}+\frac{1}{4 \sigma}\right)\left\|\eta^{t}\right\|_{\mu, 2}^{2} \\
& -\left(\frac{1}{2}-\sigma\left(\mu_{0}+d_{1}\right)\right)\|\Delta u(t)\|_{2}^{2},
\end{aligned}
$$

onde $d_{2}>0$ menciona a constante de imersão $V_{1} \hookrightarrow V_{0}$. Portanto, fixando $\sigma>0$ pequeno o suficiente, tomando por exemplo $0<\sigma \leq \frac{1}{2\left(\mu_{0}+d_{1}\right)}$, e considerando as constantes positivas

$$
C_{2}=\frac{3}{2} d_{2}+\frac{1}{4 \sigma}>0 \quad \text { e } \quad C_{3}=\frac{1}{2}+\frac{1}{4 \sigma}>0,
$$

então a estimativa (3.131) segue, como desejado. Isto finaliza a prova do Lema 3.4.3.

Agora aplicaremos os lemas acima para concluir a existência de um conjunto absorvente limitado para o semigrupo $S(t)$ em $\mathcal{H}$.

De fato, tomando $\varepsilon_{0}=\min \left\{\frac{1}{2 C_{1}}, \varepsilon_{1}\right\}$, então para todo $\varepsilon \leq \varepsilon_{0}$, segue do Lema 3.4.2 que

$$
\frac{1}{2} E(t)-\varepsilon C_{1}\left(\frac{1}{\lambda_{1}}\|h\|_{2}^{2}+k_{1}|\Omega|\right) \leq E_{\varepsilon}(t) \leq \frac{3}{2} E(t)+\varepsilon C_{1}\left(\frac{1}{\lambda_{1}}|| h \|_{2}^{2}+k_{1}|\Omega|\right),
$$

para $t \geq 0$. Combinando a segunda desigualdade em (3.133) com o Lema 3.4.3 vem que

$$
\frac{d}{d t} E_{\varepsilon}(t) \leq-\frac{2}{3} \varepsilon E_{\varepsilon}(t)+\frac{2}{3} \varepsilon^{2} C_{1}\left(\frac{1}{\lambda_{1}}|| h \|_{2}^{2}+k_{1}|\Omega|\right),
$$

o que implica em

$$
E_{\varepsilon}(t) \leq E_{\varepsilon}(0) e^{-\frac{2}{3} \varepsilon t}+\varepsilon C_{1}\left(\frac{1}{\lambda_{1}}\|h\|_{2}^{2}+k_{1}|\Omega|\right), \quad t \geq 0 .
$$

Usando novamente (3.133), resulta que

$$
E(t) \leq 3 E(0) e^{-\frac{2}{3} \varepsilon t}+6 \varepsilon C_{1}\left(\frac{1}{\lambda_{1}}\|h\|_{2}^{2}+k_{1}|\Omega|\right), \quad t \geq 0 .
$$


Em virtude da estimativa inicial (3.126), concluimos de (3.134) que

$$
\left\|\left(u(t), u_{t}(t), \eta^{t}\right)\right\|_{\mathcal{H}}^{2} \leq C E(0) e^{-\delta t}+C\left(\frac{1}{\lambda_{1}}\|h\|_{2}^{2}+k_{1}|\Omega|\right)
$$

onde $\delta=\frac{2}{3} \varepsilon>0$ é pequeno e $C=4 \max \left\{3,1+6 \varepsilon C_{1}\right\}>0$ são constantes que independem do tempo e dos dados iniciais.

Finalmente, tomando a bola fechada $\mathcal{B} \subset \mathcal{H}$ centrada em zero (vetor nulo em $\mathcal{H}$ ) e raio

$$
R=\sqrt{2 C\left(\frac{1}{\lambda_{1}}\|h\|_{2}^{2}+k_{1}|\Omega|\right)},
$$

segue de (3.135) que $\mathcal{B}$ é um conjunto absorvente limitado para o semigrupo $S(t)$ definido em (3.123).

De fato, seja $B \subset \mathcal{H}$ um subconjunto limitado e considere $z_{0}=\left(u_{0}, u_{1}, \eta_{0}\right) \in B$. Assim, existe $T_{0}=T_{0}(B) \geq 0$ suficientemente grande tal que

$$
E(0) e^{-\delta t} \leq\left(\frac{1}{\lambda_{1}}\|h\|_{2}^{2}+k_{1}|\Omega|\right), \quad \forall t \geq T_{0} .
$$

Logo, a estimativa (3.135) implica que

$$
\left\|S(t) z_{0}\right\|_{\mathcal{H}}=\left\|\left(u(t), u_{t}(t), \eta^{t}\right)\right\|_{\mathcal{H}} \leq R, \quad \forall t \geq T_{0},
$$

de onde vem que $S(t) z_{0} \in \mathcal{B}$ para $t \geq T_{0}$. Com isto,

$$
S(t) B \subset \mathcal{B}, \quad \forall t \geq T_{0}
$$

Portanto, $\mathcal{B}$ é um conjunto absorvente para $S(t)$, que é claramente limitado em $\mathcal{H}$. Isto conclui a prova da Proposição 3.4.1.

Observação 3.4.1. Assumindo que $h \equiv 0$ e que $k_{1}=0$ em (3.12), então o sistema (3.5)(3.8) é exponencialmente estável. Com efeito, Supondo que a função $h$ é identicamente nula e que $k_{1}=0$, então segue diretamente de (3.134) que a energia $E(t)$ definida em (3.124) decai exponencialmente. Em virtude da estimativa (3.126) segue que as soluções de (3.5)-(3.8) possuem o mesmo comportamento ao longo do tempo.

Observação 3.4.2. Se o conjunto $\mathcal{B}:=\left\{z \in \mathcal{H} \mid\|z\|_{\mathcal{H}} \leq R\right\}$ é um conjunto absorvente limitado para o sistema dinâmico $(\mathcal{H}, S(t))$, então existe $T_{\mathcal{B}}$ tal que

$$
S(t) \mathcal{B} \subset \mathcal{B}, \quad \forall t \geq T_{\mathcal{B}}
$$

Isto implica que o conjunto

$$
\mathcal{B}_{0}:=\bigcup_{t \geq T_{B}} S(t) \mathcal{B}
$$

é um conjunto absorvente limitado positivamente invariante e $\mathcal{B}_{0} \subset \mathcal{B}$. 
Outra consequência imediata da Proposição 3.4.1 é que as soluções do problema (3.5)-(3.8) são globalmente limitadas (na norma de $\mathcal{H}$ ) quando os dados iniciais são tomados em um subconjunto limitado de $\mathcal{H}$. Cabe lembrar que esta limitação global também é fornecida pela estimativa a priori I obtida na seção 3.3. Vamos registrar esses fatos por meio do seguinte resultado:

Lema 3.4.4. Sob as hipóteses do Teorema 3.3.1, seja $z=\left(u, u_{t}, \eta\right)$ uma solução do problema (3.5)(3.8) com condição inicial $\left(u_{0}, u_{1}, \eta_{0}\right) \in B$, onde $B$ é um subconjunto limitado de $\mathcal{H}$. Então, existe uma constante $C_{B}>0$ tal que

$$
\|z(t)\|_{\mathcal{H}} \leq C_{B}, \quad \forall t \geq 0
$$

Prova. Pela Proposição 3.4.1 existe $T_{B} \geq 0$ tal que $z(t) \in \mathcal{B}$ para todo $t \geq T_{B}$, onde $\mathcal{B}$ é um conjunto absorvente limitado, digamos com constante de limitação $R>0$. Então,

$$
\|z(t)\|_{\mathcal{H}} \leq R, \quad \forall t \geq T_{B}
$$

Por outro lado, pela conclusão (3.18) do Teorema 3.3.1, existe uma constante $R_{0}>0$ tal que

$$
\|z(t)\|_{\mathcal{H}} \leq R_{0}, \quad \forall t \in\left[0, T_{B}\right)
$$

Portanto, combinando (3.136) e (3.137) a conclusão do Lema 3.4.4 segue, tomando por exemplo

$$
C_{B}=\left\{\begin{array}{lll}
R_{0} & \text { se } & t \in\left[0, T_{B}\right), \\
R & \text { se } & t \in\left[T_{B}, \infty\right) .
\end{array}\right.
$$

Observação 3.4.3. A mesma conclusão do Lema 3.4.4 vale, em particular, substituindo o subconjunto limitado $B$ pelo conjunto absorvente $\mathcal{B}$, uma vez que este último é limitado. Mais precisamente, se $\left(u, u_{t}, \eta\right)$ é uma solução do problema (3.5)-(3.8) com condição inicial $\left(u_{0}, u_{1}, \eta_{0}\right) \in \mathcal{B}$, então existe uma constante $C_{\mathcal{B}}>0$ tal que

$$
\left\|\left(u(t), u_{t}(t), \eta^{t}\right)\right\|_{\mathcal{H}} \leq C_{\mathcal{B}}, \quad \forall t \geq 0 .
$$

\subsection{Um resultado de estabilização}

Nesta seção, e nas seguintes, sempre denotaremos por $\mathcal{B}$ o conjunto absorvente limitado obtido na seção 3.4 correspondente ao $C_{0}$-semigrupo $S(t)$ definido por (3.123).

No que segue, provaremos uma desigualdade, a qual chamaremos de desigualdade de estabilização, que será importante para determinar a existência de um atrator global para o sistema dinâmico $S(t)$ associado ao problema (3.5)-(3.8). Mais ainda, esta desigualdade de estabilização também será eficaz para concluir que o atrator global possui dimensão fractal finita. Mais precisamente, temos: 
Proposição 3.5.1. Sob as hipóteses do Teorema 3.3.1, sejam $z_{i}=\left(u^{i}, u_{t}^{i}, \eta^{i}\right)$ duas soluções do problema (3.5)-(3.8) tais que $z_{i}(0)=\left(u_{0}^{i}, u_{1}^{i}, \eta_{0}^{i}\right) \in \mathcal{B}$, para $i=1,2$. Então, vale a seguinte estimativa:

$$
\begin{aligned}
\| z_{1}(t)- & z_{2}(t)\left\|_{\mathcal{H}}^{2} \leq 3 e^{-\nu t}\right\| z_{1}(0)-z_{2}(0) \|_{\mathcal{H}}^{2} \\
& +K_{\mathcal{B}} \int_{0}^{t} e^{-\nu(t-\tau)}\left(\left\|\nabla\left(u^{1}(\tau)-u^{2}(\tau)\right)\right\|_{2(p-1)}^{2}+\left\|u^{1}(\tau)-u^{2}(\tau)\right\|_{2(\rho+1)}^{2}\right) d \tau,
\end{aligned}
$$

para todo $t \geq 0$, onde $\nu>0$ é uma constante pequena e $K_{\mathcal{B}}>0$ é uma constante dependendo de $\mathcal{B}$.

Prova. Pondo $w=u^{1}-u^{2}$ e $\zeta=\eta^{1}-\eta^{2}$, então a função $\left(w, w_{t}, \zeta\right)=z_{1}-z_{2}$ é solução (no sentido do Teorema 3.3.1) do seguinte problema

$$
\begin{aligned}
& w_{t t}+\Delta^{2} w-\Delta_{p} u^{1}+\Delta_{p} u^{2}+\int_{0}^{\infty} \mu(s) \Delta^{2} \zeta^{t}(s) d s-\Delta w_{t}+f\left(u^{1}\right)-f\left(u^{2}\right)=0 \\
& \zeta_{t}=-\zeta_{s}+w_{t}
\end{aligned}
$$

com condição inicial

$$
\left(w(0), w_{t}(0), \zeta^{0}\right)=z_{1}(0)-z_{2}(0) .
$$

Analogamente como obtido em (3.117), segue que

$$
\begin{aligned}
\frac{1}{2} \frac{d}{d t}\left\{\|\Delta w(t)\|_{2}^{2}+\left\|w_{t}(t)\right\|_{2}^{2}+\left\|\zeta^{t}\right\|_{\mu, 2}^{2}\right\}+\left\|\nabla w_{t}(t)\right\|_{2}^{2} \\
\quad=\left\langle\Delta_{p} u^{1}(t)-\Delta_{p} u^{2}(t), w_{t}(t)\right\rangle-\left(f\left(u^{1}(t)\right)-f\left(u^{2}(t)\right), w_{t}(t)\right)-\left(\zeta^{t}, \zeta_{s}^{t}\right)_{\mu, 2} .
\end{aligned}
$$

Também, como já determinamos em (3.120), vale a estimativa

$$
-\left(\zeta^{t}, \zeta_{s}^{t}\right)_{\mu, 2} \leq-\frac{k_{2}}{2} \int_{0}^{\infty} \mu(s)\left\|\Delta \zeta^{t}(s)\right\|_{2}^{2} d s=-\frac{k_{2}}{2}\left\|\zeta^{t}\right\|_{\mu, 2}^{2} .
$$

No que segue, usaremos o mesmo parâmetro $C_{\mathcal{B}}$ para denotar diferentes constantes positivas que dependem do conjunto absorvente limitado $\mathcal{B}$.

Usando o Lema 1.3.1, a desigualdade de Hölder generalizada com $\frac{p-2}{2(p-1)}+\frac{1}{2(p-1)}+\frac{1}{2}=1$, a condição (3.9) e a estimativa global (3.138), vem que

$$
\begin{aligned}
\mid\left\langle\Delta_{p} u^{1}(t)-\right. & \left.\Delta_{p} u^{2}(t), w_{t}(t)\right\rangle \mid \\
& \leq\left.\int_{\Omega}|| \nabla u^{1}(t)\right|^{p-2} \nabla u^{1}(t)-\left|\nabla u^{2}(t)\right|^{p-2} \nabla u^{2}(t)|| \nabla w_{t}(t) \mid d x \\
& \leq M_{p} \int_{\Omega}\left(\left|\nabla u^{1}(t)\right|^{p-2}+\left|\nabla u^{2}(t)\right|^{p-2}\right)|\nabla w(t)|\left|\nabla w_{t}(t)\right| d x \\
& \leq M_{p}\left(\left\|\nabla u^{1}(t)\right\|_{2(p-1)}^{p-2}+\left\|\nabla u^{2}(t)\right\|_{2(p-1)}^{p-2}\right)\|\nabla w(t)\|_{2(p-1)}\left\|\nabla w_{t}(t)\right\|_{2} \\
& \leq C_{\mathcal{B}}\|\nabla w(t)\|_{2(p-1)}\left\|\nabla w_{t}(t)\right\|_{2} .
\end{aligned}
$$

Logo, pela desigualdade de Young existe uma constante $C_{\mathcal{B}}>0$ tal que

$$
\left|\left\langle\Delta_{p} u^{1}(t)-\Delta_{p} u^{2}(t), w_{t}(t)\right\rangle\right| \leq \frac{C_{\mathcal{B}}}{2}\|\nabla w(t)\|_{2(p-1)}^{2}+\frac{1}{4}\left\|\nabla w_{t}(t)\right\|_{2}^{2} .
$$


Mais ainda, como $\frac{\rho}{2(\rho+1)}+\frac{1}{2(\rho+1)}+\frac{1}{2}=1$, então novamente pela desigualdade de Hölder generalizada, das condições (3.10)-(3.11), da estimativa (3.138) e pela deigualdade de Poincaré, obtemos

$$
\begin{aligned}
\mid\left(f\left(u^{1}(t)\right)\right. & \left.-f\left(u^{2}(t)\right), w_{t}(t)\right) \mid \\
& \leq k_{0} \int_{\Omega}\left(1+\left|u^{1}(t)\right|^{\rho}+\left|u^{2}(t)\right|^{\rho}\right)|w(t)|\left|w_{t}(t)\right| d x \\
& \leq k_{0}\left(|\Omega|^{\frac{\rho}{2(\rho+1)}}+\left\|u^{1}(t)\right\|_{2(\rho+1)}^{\rho}+\left\|u^{2}(t)\right\|_{2(\rho+1)}^{\rho}\right)\|w(t)\|_{2(\rho+1)}\left\|w_{t}(t)\right\|_{2} \\
& \leq C_{\mathcal{B}}\|w(t)\|_{2(\rho+1)}\left\|\nabla w_{t}(t)\right\|_{2} .
\end{aligned}
$$

Novamente pela desigualdade de Young, existe uma constante $C_{\mathcal{B}}>0$ tal que

$$
\left|\left(f\left(u^{1}(t)\right)-f\left(u^{2}(t)\right), w_{t}(t)\right)\right| \leq \frac{C_{\mathcal{B}}}{2}\|w(t)\|_{2(\rho+1)}^{2}+\frac{1}{4}\left\|\nabla w_{t}(t)\right\|_{2}^{2} .
$$

Substituindo (3.144)-(3.146) em (3.143), então

$$
\frac{d}{d t} F(t) \leq-\left\|\nabla w_{t}(t)\right\|_{2}^{2}-k_{2}\left\|\zeta^{t}\right\|_{\mu, 2}^{2}+C_{\mathcal{B}}\left(\|\nabla w(t)\|_{2(p-1)}^{2}+\|w(t)\|_{2(\rho+1)}^{2}\right),
$$

onde denotamos

$$
F(t)=\|\Delta w(t)\|_{2}^{2}+\left\|w_{t}(t)\right\|_{2}^{2}+\left\|\zeta^{t}\right\|_{\mu, 2}^{2}:=\left\|z_{1}(t)-z_{2}(t)\right\|_{\mathcal{H}}^{2}, \quad t \geq 0 .
$$

Agora consideremos a perturbação

$$
F_{\varepsilon}(t)=F(t)+\varepsilon \Phi(t), \quad \varepsilon>0,
$$

onde $\varepsilon>0$ será fixado depois e

$$
\Phi(t)=\int_{\Omega} w_{t}(t) w(t) d x
$$

A seguir apresentaremos dois lemas que nos permitirão concluir o desejado.

Lema 3.5.2. Existe uma constante $C_{1}>0$ tal que

$$
\left|F_{\varepsilon}(t)-F(t)\right| \leq \varepsilon C_{1} F(t), \quad \forall t \geq 0, \forall \varepsilon>0 .
$$

Prova. Segue diretamente da construção acima, usando argumentos análogos ao Lema 3.4.2.

Lema 3.5.3. Existem constantes $\varepsilon_{1}>0$ e $C_{\mathcal{B}}>0$ tais que

$$
\frac{d}{d t} F_{\varepsilon}(t) \leq-\frac{\varepsilon}{2} F(t)+C_{\mathcal{B}}\left(\|\nabla w(t)\|_{2(p-1)}^{2}+\|w(t)\|_{2(\rho+1)}^{2}\right), \quad \forall t \geq 0, \forall \varepsilon \in\left(0, \varepsilon_{1}\right] .
$$

Prova. É suficiente provar que existem constantes $C_{2}, C_{3}>0$ e uma constante $C_{\mathcal{B}}>0$, dependendo do conjunto absorvente $\mathcal{B}$, tais que

$$
\frac{d}{d t} \Phi(t) \leq-\frac{1}{2} F(t)+C_{2}\left\|\nabla w_{t}(t)\right\|_{2}^{2}+C_{3}\left\|\zeta^{t}\right\|_{\mu, 2}^{2}+C_{\mathcal{B}}\left(\|\nabla w(t)\|_{2(p-1)}^{2}+\|w(t)\|_{2(\rho+1)}^{2}\right) .
$$


De fato, combinando esta estimativa com (3.147), escolhendo $\varepsilon_{1}=\min \left\{\frac{1}{C_{2}}, \frac{k_{2}}{C_{3}}\right\}>0$ e tomando $\varepsilon \in\left(0, \varepsilon_{1}\right]$, então

$$
\begin{aligned}
\frac{d}{d t} F_{\varepsilon}(t)= & \frac{d}{d t} F(t)+\varepsilon \frac{d}{d t} \Phi(t) \\
\leq & -\frac{\varepsilon}{2} F(t)-\left(1-\varepsilon C_{2}\right)\left\|\nabla w_{t}(t)\right\|_{2}^{2}-\left(k_{2}-\varepsilon C_{3}\right)\left\|\zeta^{t}\right\|_{\mu, 2}^{2} \\
& +(1+\varepsilon) C_{\mathcal{B}}\left(\|\nabla w(t)\|_{2(p-1)}^{2}+\|w(t)\|_{2(\rho+1)}^{2}\right) \\
\leq & -\frac{\varepsilon}{2} F(t)+\left(1+\varepsilon_{1}\right) C_{\mathcal{B}}\left(\|\nabla w(t)\|_{2(p-1)}^{2}+\|w(t)\|_{2(\rho+1)}^{2}\right) .
\end{aligned}
$$

Isto mostra o Lema 3.5.3 para alguma constante $C_{\mathcal{B}}>0$ (no lugar de $\left(1+\varepsilon_{1}\right) C_{\mathcal{B}}$ ).

No que segue, vamos mostrar a veracidade de (3.150). Com efeito, derivando a função em (3.149), usando a equação (3.140) no sentido fraco, adicionando e subtraindo $\frac{1}{2} F(t)$ na expressão resultante, obtemos

$$
\frac{d}{d t} \Phi(t)=-\frac{1}{2} F(t)+\frac{3}{2}\left\|w_{t}(t)\right\|_{2}^{2}-\frac{1}{2}\|\Delta w(t)\|_{2}^{2}+\frac{1}{2}\left\|\zeta^{t}\right\|_{\mu, 2}^{2}+\sum_{j=1}^{4} L_{j}
$$

onde

$$
\begin{aligned}
& L_{1}=-\int_{0}^{\infty} \mu(s)\left(\Delta \zeta^{t}(s), \Delta w(t)\right) d s, \\
& L_{2}=-\int_{\Omega} \nabla w_{t}(t) \cdot \nabla w(t) d x, \\
& L_{3}=\left\langle\Delta_{p} u^{1}(t)-\Delta_{p} u^{2}(t), w(t)\right\rangle, \\
& L_{4}=-\left(f\left(u^{1}(t)\right)-f\left(u^{2}(t)\right), w(t)\right) .
\end{aligned}
$$

Agora vamos estimar os termos $L_{1}, L_{2}, L_{3}$ e $L_{4}$. Em primeiro lugar, procedendo exatamente como na estimativa de $I_{2}$ e $I_{3}$ feitas na seção 3.4 (ver prova do Lema 3.4.3), derivamos pela desigualdade de Young com $\sigma>0$ que:

$$
\left|L_{1}\right| \leq \sigma \mu_{0}\|\Delta w(t)\|_{2}^{2}+\frac{1}{4 \sigma}\left\|\zeta^{t}\right\|_{\mu, 2}^{2}
$$

e

$$
\left|L_{2}\right| \leq \sigma d_{1}\|\Delta w(t)\|_{2}^{2}+\frac{1}{4 \sigma}\left\|\nabla w_{t}(t)\right\|_{2}^{2},
$$

onde $d_{1}>0$ é a constante de imersão $V_{2} \hookrightarrow V_{1}$.

Em segundo lugar, utilizando os mesmos argumentos para obter (3.145)-(3.146), e usando $w$ no lugar de $w_{t}$, obtemos mais as seguintes estimativas:

$$
\left|L_{3}\right| \leq C_{\mathcal{B}}\|\nabla w(t)\|_{2(p-1)}^{2}
$$

$\mathrm{e}$

$$
\left|L_{4}\right| \leq C_{\mathcal{B}}\|w(t)\|_{2(\rho+1)}^{2},
$$

para alguma constante $C_{\mathcal{B}}>0$ dependendo do conjunto absorvente limitado $\mathcal{B}$. 
Retornando a (3.151) e substituindo estas quatro últimas estimativas, chegamos a

$$
\begin{aligned}
\frac{d}{d t} \Phi(t) \leq & -\frac{1}{2} F(t)+\left(\frac{3}{2} d_{2}+\frac{1}{4 \sigma}\right)\left\|\nabla w_{t}(t)\right\|_{2}^{2}+\left(\frac{1}{2}+\frac{1}{4 \sigma}\right)\left\|\zeta^{t}\right\|_{\mu, 2}^{2} \\
& +C_{\mathcal{B}}\left(\|\nabla w(t)\|_{2(p-1)}^{2}+\|w(t)\|_{2(\rho+1)}^{2}\right)-\left(\frac{1}{2}-\sigma\left(\mu_{0}+d_{1}\right)\right)\|\Delta w(t)\|_{2}^{2},
\end{aligned}
$$

onde $d_{2}>0$ é a constante de imersão $V_{1} \hookrightarrow V_{0}$. Portanto, tomando

$$
\sigma \leq \frac{1}{2\left(\mu_{0}+2 d_{1}\right)}, \quad C_{2}=\frac{3}{2} d_{2}+\frac{1}{4 \sigma} \quad \text { e } \quad C_{3}=\frac{1}{2}+\frac{1}{4 \sigma},
$$

a desigualdade (3.150) fica provada. Isto termina a prova do Lema 3.5.3.

Com estes dois lemas, estamos aptos a provar a desigualdade de estabilização (3.139) como segue. Definamos $\varepsilon_{0}=\min \left\{\frac{1}{2 C_{1}}, \varepsilon_{1}\right\}$ e considere $\varepsilon \leq \varepsilon_{0}$. Assim, o Lemma 3.5.2 implica que

$$
\frac{1}{2} F(t) \leq F_{\varepsilon}(t) \leq \frac{3}{2} F(t), \quad t \geq 0 .
$$

Combinando, de forma padrão, o Lema 3.5.3 com a segunda desigualdade em (3.152), resulta que

$$
F_{\varepsilon}(t) \leq e^{-\varepsilon t / 3} F_{\varepsilon}(0)+C_{\mathcal{B}} \int_{0}^{t} e^{-\varepsilon(t-\tau) / 3}\left(\|\nabla w(\tau)\|_{2(p-1)}^{2}+\|w(\tau)\|_{2(\rho+1)}^{2}\right) d \tau, \quad t \geq 0 .
$$

Usando novamente (3.152), concluimos que

$$
F(t) \leq 3 e^{-\nu t} F(0)+K_{\mathcal{B}} \int_{0}^{t} e^{-\nu(t-\tau)}\left(\|\nabla w(\tau)\|_{2(p-1)}^{2}+\|w(\tau)\|_{2(\rho+1)}^{2}\right) d \tau, \quad t \geq 0,
$$

onde $\nu=\varepsilon / 3>0$ é uma constante positiva pequena e $K_{\mathcal{B}}=2 C_{\mathcal{B}}>0$ é uma constante dependendo de $\mathcal{B}$. Mantendo em mente a identidade (3.148) e que $w=u^{1}-u^{2}$, então a estimativa (3.153) implica na desigualdade de estabilização (3.139), o que encerra a prova da Proposição 3.5.1.

Observação 3.5.1. A mesma conclusão da Proposição 3.5.1 permanece verdadeira se substituirmos o conjunto absorvente limitado $\mathcal{B}$ por qualquer outro subconjunto limitado $B \subset \mathcal{H}$.

\subsection{Atrator Global}

De acordo com a teoria geral relembrada na seção 1.6 do Capítulo 1, para concluir que o sistema dinâmico dissipativo $(\mathcal{H}, S(t))$ associado ao problema (3.5)-(3.8) possui um atrator global, devemos mostrar que $S(t)$ é assintoticamente suave (ver Proposição 1.6.1). Para tanto, a fim de determinar que $S(t)$ é assintoticamente suave, mostraremos uma propriedade de estabilização conforme requer a Proposição 1.6.2. Veremos mais adiante que tal propriedade é, quase que, uma consequência imediata da desigualdade de estabilização determinada na Proposição 3.5.1.

O próximo resultado determina um dos principais objetivos deste capítulo, a saber, o de provar a existência de um atrator global para o semigrupo $S(t)$ definido por (3.123). Em seguida, estudaremos a dimensão fractal deste atrator dentro do espaço de fase infinito-dimensional $\mathcal{H}$. 
Teorema 3.6.1. Sob as hipóteses do Teorema 3.3.1, o semigrupo $S(t)$ associado ao problema (3.5)(3.8) possui um atrator global compacto $\mathcal{A}$ em $\mathcal{H}$.

Prova. Como o sistema dinâmico $(\mathcal{H}, S(t))$ é dissipativo, então a conclusão do teorema segue se mostrarmos que $S(t)$ é assintoticamente suave. Faremos isto mostrando o semigrupo $S(t)$ definido por (3.123) satisfaz as condições da Proposição 1.6.2.

Para $i=1,2$, consideremos $z_{0}^{i}=\left(u_{0}^{i}, u_{1}^{i}, \eta_{0}^{i}\right) \in B$, onde $B \subset \mathcal{H}$ é um conjunto limitado positivamente invariante. Assim, a Proposição 3.5.1 (ver também Observação 3.5.1) implica que existem constantes $\nu>0$ e $C_{B}>0$ tais que

$$
\begin{aligned}
& \left\|S(t) z_{0}^{1}-S(t) z_{0}^{2}\right\|_{\mathcal{H}}^{2} \leq 3 e^{-\nu t}\left\|z_{0}^{1}-z_{0}^{2}\right\|_{\mathcal{H}}^{2} \\
& \quad+C_{B} \int_{0}^{t} e^{-\nu(t-\tau)}\left(\left\|\nabla\left(u^{1}(\tau)-u^{2}(\tau)\right)\right\|_{2(p-1)}^{2}+\left\|u^{1}(\tau)-u^{2}(\tau)\right\|_{2(\rho+1)}^{2}\right) d \tau,
\end{aligned}
$$

para todo $t \geq 0$, onde $S(t) z_{0}^{i}=\left(u^{i}(t), u_{t}^{i}(t), \eta^{i, t}\right)$ são as soluções do problema (3.5)-(3.8) correspondentes aos dados iniciais, as quais são globalmente limitadas na norma de $\mathcal{H}$ conforme o Lema 3.4.4. Em particular, segue também que as funções $u^{1}(t)$ e $u^{2}(t)$ são (globalmente) limitadas na norma de $V_{2}=H^{2}(\Omega) \cap H_{0}^{1}(\Omega)$.

No que segue, vamos estimar a norma

$$
\left\|\nabla\left(u^{1}(t)-u^{2}(t)\right)\right\|_{2(p-1)}^{2}+\left\|u^{1}(t)-u^{2}(t)\right\|_{2(\rho+1)}^{2}
$$

dentro da integral em (3.154) por uma norma em $V_{0}=L^{2}(\Omega)$. Para isso, usaremos a desigualdade de Gagliardo-Nirenberg (ver Teorema 1.2.4), e sua consequência, a desigualdade de interpolação dada pelo Teorema 1.2.5. A mesma notação $C_{B}$ será usada para denotar diferentes constantes positivas.

Em primeiro lugar, para cada $N \in \mathbb{N}$, definamos

$$
\theta_{1}=\frac{1}{2}+\frac{N}{4}\left(1-\frac{1}{p-1}\right)
$$

Usando a hipótese (3.9), segue que $\theta_{1}$ satisfaz as condições

$$
\frac{1}{2} \leq \theta_{1} \leq 1 \quad \text { e } \quad 1-\frac{N}{2(p-1)}=\theta_{1}\left(2-\frac{N}{2}\right)-\frac{N}{2}\left(1-\theta_{1}\right) .
$$

Logo, pela desigualdade de Gagliardo-Nirenberg obtemos que

$$
\begin{aligned}
\left\|\nabla\left(u^{1}(t)-u^{2}(t)\right)\right\|_{2(p-1)} & \leq C_{\theta_{1}}\left\|\Delta\left(u^{1}(t)-u^{2}(t)\right)\right\|_{2}^{1-\theta_{1}}\left\|u^{1}(t)-u^{2}(t)\right\|_{2}^{\theta_{1}} \\
& \leq C_{B}\left\|u^{1}(t)-u^{2}(t)\right\|_{2}^{\theta_{1}}
\end{aligned}
$$

para alguma constante $C_{B}>0$.

Agora, note que a hipótese (3.11) infere nas seguintes desigualdades

$$
2<2(\rho+1)\left\{\begin{array}{lll}
<\infty & \text { se } & 1 \leq N \leq 4 \\
\leq \frac{2 N}{N-4} & \text { se } & N \geq 5
\end{array}\right.
$$


Assim sendo, definindo $\theta_{2}=\frac{N}{4}\left(1-\frac{1}{\rho+1}\right)$, segue pelo Teorema 1.2.5 que

$$
\begin{aligned}
\left\|u^{1}(t)-u^{2}(t)\right\|_{2(\rho+1)} & \leq C_{\theta_{2}}\left\|\Delta\left(u^{1}(t)-u^{2}(t)\right)\right\|_{2}^{1-\theta_{2}}\left\|u^{1}(t)-u^{2}(t)\right\|_{2}^{\theta_{2}} \\
& \leq C_{B}\left\|u^{1}(t)-u^{2}(t)\right\|_{2}^{\theta_{2}}
\end{aligned}
$$

para alguma constante $C_{B}>0$. Combinando as estimativas (3.155) e (3.156) e usando novamente que $u^{1}(t), u^{2}(t)$ são limitadas em $V_{2} \hookrightarrow V_{0}$, então existe uma constante $C_{B}>0$ dependendo do subconjunto limitado $B$ tal que

$$
\begin{aligned}
\| \nabla\left(u^{1}(t)\right. & \left.-u^{2}(t)\right)\left\|_{2(p-1)}^{2}+\right\| u^{1}(t)-u^{2}(t) \|_{2(\rho+1)}^{2} \\
& \leq C_{B}\left(\left\|u^{1}(t)-u^{2}(t)\right\|_{2}^{2 \theta_{1}}+\left\|u^{1}(t)-u^{2}(t)\right\|_{2}^{2 \theta_{2}}\right) \\
& =C_{B}\left(\left\|u^{1}(t)-u^{2}(t)\right\|_{2}^{2\left(\theta_{1}-\theta\right)}+\left\|u^{1}(t)-u^{2}(t)\right\|_{2}^{2\left(\theta_{2}-\theta\right)}\right)\left\|u^{1}(t)-u^{2}(t)\right\|_{2}^{2 \theta} \\
& \leq C_{B}\left\|u^{1}(t)-u^{2}(t)\right\|_{2}^{2 \theta},
\end{aligned}
$$

onde consideramos $\theta=\min \left\{\theta_{1}, \theta_{2}\right\}$. Em resumo,

$$
\left\|\nabla\left(u^{1}(t)-u^{2}(t)\right)\right\|_{2(p-1)}^{2}+\left\|u^{1}(t)-u^{2}(t)\right\|_{2(\rho+1)}^{2} \leq C_{B}\left\|u^{1}(t)-u^{2}(t)\right\|_{2}^{2 \theta},
$$

para alguma constante $C_{B}>0$. Logo, substituindo (3.157) em (3.154) vem que

$$
\left\|S(t) z_{0}^{1}-S(t) z_{0}^{2}\right\|_{\mathcal{H}}^{2} \leq 3 e^{-\nu t}\left\|z_{0}^{1}-z_{0}^{2}\right\|_{\mathcal{H}}^{2}+C_{B}^{2} \int_{0}^{t} e^{-\nu(t-\tau)}\left\|u^{1}(\tau)-u^{2}(\tau)\right\|_{2}^{2 \theta} d \tau,
$$

para todo $t \geq 0$, o que implica em

$$
\left\|S(t) z_{0}^{1}-S(t) z_{0}^{2}\right\|_{\mathcal{H}}^{2} \leq 3 e^{-\nu t}\left\|z_{0}^{1}-z_{0}^{2}\right\|_{\mathcal{H}}^{2}+\frac{C_{B}^{2}}{\nu} \sup _{0 \leq \tau \leq t}\left\|u^{1}(\tau)-u^{2}(\tau)\right\|_{2}^{2 \theta},
$$

ou ainda,

$$
\left\|S(t) z_{0}^{1}-S(t) z_{0}^{2}\right\|_{\mathcal{H}} \leq \sqrt{6} e^{-\nu t / 2}\left\|z_{0}^{1}-z_{0}^{2}\right\|_{\mathcal{H}}+\sqrt{\frac{2}{\nu}} C_{B} \sup _{0 \leq \tau \leq t}\left\|u^{1}(\tau)-u^{2}(\tau)\right\|_{2}^{\theta},
$$

para todos $t \geq 0$ e $z_{0}^{1}, z_{0}^{2} \in B$. Agora definamos:

(i) $Q(t):=\sqrt{6} e^{-\nu t / 2}, \quad t \geq 0$.

(ii) $K_{B}(t):=\sqrt{\frac{2}{\nu}} C_{B}, \quad t \geq 0$.

(iii) A função $m: \mathcal{H} \times \mathcal{H} \rightarrow \mathbb{R}$ dada por

$$
m\left(z_{0}^{1}, z_{0}^{2}\right)=\sup _{0 \leq \tau \leq t}\left\|u^{1}(\tau)-u^{2}(\tau)\right\|_{2}^{\theta}
$$

onde $u^{i}$ é a primeira componente da solução $z=\left(u^{i}, u_{t}^{i}, \eta^{i}\right)$ correspondente a $z_{0}^{i}$, para $i=1,2$. 
Com isto, obtemos diretamente que $Q(t) \rightarrow 0$ quando $t \rightarrow \infty, K_{B}(t) \equiv K_{B}>0$ é uma constante positiva, $m\left(z_{0}^{1}, z_{0}^{2}\right)$ é uma pseudométrica e, além disso, podemos reescrever (3.158) como

$$
\left\|S(t) z_{0}^{1}-S(t) z_{0}^{2}\right\|_{\mathcal{H}} \leq Q(t)\left\|z_{0}^{1}-z_{0}^{2}\right\|_{\mathcal{H}}+K_{B} m\left(z_{0}^{1}, z_{0}^{2}\right) ; \quad \forall t \geq 0, \quad \forall z_{0}^{1}, z_{0}^{2} \in B .
$$

Resta verificar que a pseudométrica $m\left(z_{0}^{1}, z_{0}^{2}\right)$ é pré-compacta. Contudo, esta condição é satisfeita em virtude da limitação global (em norma) da função $z(t)=\left(u(t), u_{t}(t), \eta^{t}\right)$ em $\mathcal{H}$ e da inclusão compacta $V_{2} \hookrightarrow V_{0}$, ver Teorema 4.7.8 em Hale [55], (ver também Simon [89, Lema 1]).

Portanto, a Proposição 1.6.2 afirma que o semigrupo $S(t)$ definido por (3.123) é assintoticamente suave e, como consequência, a Proposição 1.6.1 assegura que $S(t)$ possui um atrator global compacto $\mathcal{A}$ em $\mathcal{H}$.

Isto conclui a prova do Teorema 3.6.1.

Observação 3.6.1. Vale a pena lembrar que o atrator global $\mathcal{A}$ para o semigrupo $S(t)$ associado ao problema (3.5)-(3.8) é único. Na verdade este fato vale de um modo mais geral, sendo proveniente da teoria abstrata para atratores. Com efeito, sejam $\mathcal{A}$ e $\mathcal{A}^{*}$ dois atratores globais para o semigrupo $S(t)$. Por definição, $\mathcal{A}$ atrai uniformemente todo subconjunto limitado de $\mathcal{H}$ e $\mathcal{A}^{*}$ é invariante sob a ação de $S(t)$. Logo,

$$
\operatorname{dist}_{H}\left(\mathcal{A}^{*}, \mathcal{A}\right)=\operatorname{dist}_{H}\left(S(t) \mathcal{A}^{*}, \mathcal{A}\right) \rightarrow 0 \text { quando } t \rightarrow+\infty,
$$

de onde segue que $\mathcal{A}^{*} \subset \mathcal{A}$. Analogamente se verifica que $\mathcal{A} \subset \mathcal{A}^{*}$, o que conclui a unicidade desejada.

\subsubsection{Dimensão fractal finita}

Tanto do ponto de vista teórico quanto do aplicado, entendemos que é interessante estudar uma propriedade que avalia, em algum aspecto, a dimensão (finita) de atratores dentro do contexto de sistemas dinâmicos infinito-dimensional. Seguindo essa linha de raciocínio, vamos abordar uma das medidas mais comuns no contexto de atratores, a saber, a dimensão fractal (seção 1.6 - Capítulo 1) para o atrator global $\mathcal{A}$ obtido no Teorema 3.6.1.

De um modo mais preciso, vamos estabelecer a seguir que o atrator global $\mathcal{A}$, correspondente ao semigrupo definido por (3.123), possui dimensão fractal finita. Para fazer isso, combinaremos a desigualdade de estabilização obtida anteriormente com a Proposição 1.6.6. Este é o nosso propósito para concluir nossos estudos neste capítulo. No entanto, para atingir tal objetivo em dimensões maiores, as hipóteses impostas sobre as constantes $p$ e $\rho$ (ver condições (3.9) e (3.11) na seção 3.2) devem ser passadas ao caso subcrítico como segue:

$$
2 \leq p<\frac{2 N-2}{N-2} \quad \text { se } \quad N \geq 3 \quad \text { e } \quad p \geq 2 \quad \text { se } \quad N=1,2
$$

$\mathrm{e}$

$$
0<\rho<\frac{4}{N-4} \quad \text { se } \quad N \geq 5 \quad \text { e } \quad \rho>0 \quad \text { se } \quad 1 \leq N \leq 4 .
$$

Em resumo, temos o seguinte resultado: 
Teorema 3.6.2. Sob as hipóteses do Teorema 3.3.1, com as condições (3.159) e (3.160) no lugar de (3.9) e (3.11), respectivamente, então o atrator global compacto $\mathcal{A}$ obtido no Teorema 3.6.1 possui dimensão fractal finita.

Prova. Da Proposição 3.5.1, existem constantes $\nu>0$ e $K_{\mathcal{A}}>0$ tais que

$$
\begin{aligned}
& \left\|S(t) z_{0}^{1}-S(t) z_{0}^{2}\right\|_{\mathcal{H}}^{2} \leq 3 e^{-\nu t}\left\|z_{0}^{1}-z_{0}^{2}\right\|_{\mathcal{H}}^{2} \\
& \quad+K_{\mathcal{A}} \int_{0}^{t} e^{-\nu(t-\tau)}\left(\left\|\nabla\left(u^{1}(\tau)-u^{2}(\tau)\right)\right\|_{2(p-1)}^{2}+\left\|u^{1}(\tau)-u^{2}(\tau)\right\|_{2(\rho+1)}^{2}\right) d \tau
\end{aligned}
$$

para todos $t \geq 0$ e $z_{0}^{i}=\left(u_{0}^{i}, u_{1}^{i}, \eta_{0}^{i}\right) \in \mathcal{A}, i=1,2$, onde $S(t) z_{0}^{i}=\left(u^{i}(t), u_{t}^{i}(t), \eta^{i, t}\right)$ são as soluções do problema (3.5)-(3.8) correspondentes aos dados iniciais, as quais são globalmente limitadas na norma de $\mathcal{H}$ conforme o Lema 3.4.4.

Antes de prosseguirmos com as contas, vamos relembrar algumas propriedades abstratas com respeito a potência fracionária do operador biharmônico, ver por exemplo Chueshov \& Lasiecka [23, 24] ou [25, Capítulo 7].

Sendo a inclusão $V_{2} \hookrightarrow V_{0}$ compacta, vamos definir o operador $A$ pela terna $\left\{V_{2}, V_{0},(\cdot, \cdot)_{V_{2}}\right\}$, isto é, mediante ao exposto na seção 1.4 do Capítulo 1 temos:

$$
\langle A u, v\rangle=(\Delta u, \Delta v), \quad \forall u, v \in V_{2}
$$

Além disso, nesse caso em particular é bem conhecido que

$$
D(A)=\left\{u \in H^{4}(\Omega) \cap H_{0}^{1}(\Omega) \mid \Delta u=0 \text { sobre } \Gamma\right\} \quad \text { e } \quad A u=\Delta^{2} u, \quad \forall u \in D(A) .
$$

Mais ainda, devido a Observação 1.4.1 o operador linear $A$ definido em (3.162) é densamente definido em $V_{0}$, auto-adjunto e definido positivo em $V_{2}$. Logo, podemos definir as potências fracionárias $A^{s}, s \in \mathbb{R}$, do operador $A$ com domínio $D\left(A^{s}\right)$ sendo espaços de Hilbert quando munido do produto interno e norma

$$
(u, v)_{D\left(A^{s}\right)}=\left(A^{s} u, A^{s} v\right) \quad \text { e } \quad\|u\|_{D\left(A^{s}\right)}=\left\|A^{s} u\right\|_{2}, \quad \forall u, v \in D\left(A^{s}\right)
$$

respectivamente. Em particular, notamos que $D\left(A^{1 / 4}\right)=H_{0}^{1}(\Omega):=V_{1}$ e também, para $s=1 / 2$,

$$
D\left(A^{1 / 2}\right)=H^{2}(\Omega) \cap H_{0}^{1}(\Omega):=V_{2} \quad \text { e } \quad A^{1 / 2} u=-\Delta u, \quad \forall u \in D\left(A^{1 / 2}\right) .
$$

Note ainda que $D\left(A^{s_{1}}\right) \hookrightarrow D\left(A^{s_{2}}\right)$ se $s_{1} \geq s_{2}$ e $D\left(A^{s_{1}}\right) \hookrightarrow \hookrightarrow D\left(A^{s_{2}}\right)$ se $s_{1}>s_{2}$, conforme o Lema 1.4.1

Retornando as contas, estimaremos abaixo a norma dentro da integral em (3.161) por uma norma em um espaço com potência fracionária do operador biharmônico. De fato, para qualquer $N \in \mathbb{N}$, segue das novas hipóteses (3.159) e (3.160) que

$$
\frac{1}{4} \leq \frac{1}{4}+\frac{N}{8}\left(1-\frac{1}{p-1}\right)<\frac{1}{2} \quad \text { e } \quad 0<\frac{N}{8}\left(1-\frac{1}{\rho+1}\right)<\frac{1}{2}
$$


respectivamente. Assim sendo, podemos escolher dois números $\gamma_{1}$ e $\gamma_{2}$ tais que

$$
\frac{1}{4}+\frac{N}{8}\left(1-\frac{1}{p-1}\right)<\gamma_{1}<\frac{1}{2} \quad \text { e } \quad \frac{N}{8}\left(1-\frac{1}{\rho+1}\right)<\gamma_{2}<\frac{1}{2},
$$

para cada $p, \rho$ e $N$ dados. Com isto, é fácil ver que $\gamma_{1} \in\left(\frac{1}{4}, \frac{1}{2}\right), \gamma_{2} \in\left(0, \frac{1}{2}\right)$ e que valem as seguintes desigualdades:

$$
1-\frac{N}{2(p-1)}<4 \gamma_{1}-\frac{N}{2} \quad \text { e } \quad 0-\frac{N}{2(\rho+1)}<4 \gamma_{2}-\frac{N}{2} .
$$

Logo, o Teorema 4.3 em Pazy [85, Capítulo 8] (ver também Teorema 1.6.1 em Henry [57, Capítulo 1]) implica nas seguintes inclusões contínuas

$$
D\left(A^{\gamma_{1}}\right) \hookrightarrow W^{1,2(p-1)}(\Omega) \quad \text { e } \quad D\left(A^{\gamma_{2}}\right) \hookrightarrow W^{0,2(\rho+1)}(\Omega)=L^{2(\rho+1)}(\Omega) .
$$

Agora, definindo $\gamma=\max \left\{\gamma_{1}, \gamma_{2}\right\} \in\left(\frac{1}{4}, \frac{1}{2}\right)$ para cada $p, \rho$ e $N$ dados, obtemos (via Lema 1.4.1) que

$$
D\left(A^{\gamma}\right) \hookrightarrow D\left(A^{\gamma_{1}}\right) \quad \text { e } \quad D\left(A^{\gamma}\right) \hookrightarrow D\left(A^{\gamma_{2}}\right)
$$

de onde segue que

$$
D\left(A^{\gamma}\right) \hookrightarrow D\left(A^{\gamma_{1}}\right) \cap D\left(A^{\gamma_{2}}\right) .
$$

Combinando esta inclusão com as inclusões em (3.163), existe uma constante $C_{\gamma}>0$ tal que

$$
\left\|\nabla\left(u^{1}(t)-u^{2}(t)\right)\right\|_{2(p-1)}^{2}+\left\|u^{1}(t)-u^{2}(t)\right\|_{2(\rho+1)}^{2} \leq C_{\gamma}\left\|A^{\gamma}\left(u^{1}(t)-u^{2}(t)\right)\right\|_{2}^{2}, \quad t \geq 0,
$$

para algum $\gamma \in\left(\frac{1}{4}, \frac{1}{2}\right)$. Substituindo (3.164) em (3.161), vem que

$$
\left\|S(t) z_{0}^{1}-S(t) z_{0}^{2}\right\|_{\mathcal{H}}^{2} \leq 3 e^{-\nu t}\left\|z_{0}^{1}-z_{0}^{2}\right\|_{\mathcal{H}}^{2}+C_{\mathcal{A}} \sup _{0 \leq \tau \leq t}\left\|A^{\gamma}\left(u^{1}(\tau)-u^{2}(\tau)\right)\right\|_{2}^{2}
$$

para todos $t \geq 0$ e $z_{0}^{1}, z_{0}^{2} \in \mathcal{A}$, onde $C_{\mathcal{A}}:=C_{\gamma} K_{\mathcal{A}} / \nu>0$ é uma constante dependendo do atrator $\mathcal{A}$ e $\gamma \in\left(\frac{1}{4}, \frac{1}{2}\right)$.

Por outro lado, segue diretamente do item (iii) do Teorema 3.3.1 que

$$
\left\|S(t) z_{0}^{1}-S(t) z_{0}^{2}\right\|_{\mathcal{H}}^{2} \leq e^{c t}\left\|z_{0}^{1}-z_{0}^{2}\right\|_{\mathcal{H}}^{2}
$$

para todos $z_{0}^{1}, z_{0}^{2} \in \mathcal{A}$ e $t \geq 0$ (uma vez que as soluções existem globalmente) e para alguma constante $c=c(\mathcal{A})>0$ dependendo de $\mathcal{A}$.

Estas duas últimas estimativas (em conjunto com as considerações a seguir) serão cruciais para determinar outras duas estimativas, as quais nos possibilitarão definir um operador e um subconjunto que satisfaz as condições da Proposição 1.6.6. Em seguida, concluiremos que o atrator global compacto $\mathcal{A}$ possui dimensão fractal finita.

Em primeiro lugar, fixamos um tempo $T^{*}>0$, que será convenientemente especificado mais adiante, e consideremos o espaço

$$
W_{1}\left(0, T^{*}\right)=\left\{w(t) \mid\|w\|_{W_{1}\left(0, T^{*}\right)}^{2}:=\int_{0}^{T^{*}}\left(\|\Delta w(t)\|_{2}^{2}+\left\|w_{t}(t)\right\|_{2}^{2}\right) d t<\infty\right\} .
$$


Agora definamos o espaço

$$
\mathcal{H}_{T^{*}}=\mathcal{H} \times W_{1}\left(0, T^{*}\right),
$$

equipado com a norma

$$
\|U\|_{\mathcal{H}_{T^{*}}}^{2}=\|\Delta u\|_{2}^{2}+\|v\|_{2}^{2}+\|\eta\|_{\mu, 2}^{2}+\|w\|_{W_{1}\left(0, T^{*}\right)}^{2}, \quad U=(u, v, \eta, w) \in \mathcal{H}_{T^{*}}
$$

Pondo $w(t)=u^{1}(t)-u^{2}(t)$ e integrando (3.165) de $T^{*}$ a $2 T^{*}$, resulta que

$$
\int_{T^{*}}^{2 T^{*}}\left\|S(t) z_{0}^{1}-S(t) z_{0}^{2}\right\|_{\mathcal{H}}^{2} d t \leq \frac{3}{\nu} e^{-\nu T^{*}}\left\|z_{0}^{1}-z_{0}^{2}\right\|_{\mathcal{H}}^{2}+C_{\mathcal{A}} T^{*} \sup _{0 \leq \tau \leq 2 T^{*}}\left\|A^{\gamma} w(\tau)\right\|_{2}^{2} .
$$

Além disso, notamos que a estimativa (3.165) vale para $T^{*}>0$, ou seja,

$$
\left\|S\left(T^{*}\right) z_{0}^{1}-S\left(T^{*}\right) z_{0}^{2}\right\|_{\mathcal{H}}^{2} \leq 3 e^{-\nu T^{*}}\left\|z_{0}^{1}-z_{0}^{2}\right\|_{\mathcal{H}}^{2}+C_{\mathcal{A}} \sup _{0 \leq \tau \leq T^{*}}\left\|A^{\gamma} w(\tau)\right\|_{2}^{2}
$$

Combinando (3.167) e (3.168) obtemos

$$
\begin{aligned}
& \left\|S\left(T^{*}\right) z_{0}^{1}-S\left(T^{*}\right) z_{0}^{2}\right\|_{\mathcal{H}}^{2}+\int_{T^{*}}^{2 T^{*}}\left\|S(t) z_{0}^{1}-S(t) z_{0}^{2}\right\|_{\mathcal{H}}^{2} d t \\
& \quad \leq 3\left(1+\nu^{-1}\right) e^{-\nu T^{*}}\left\|z_{0}^{1}-z_{0}^{2}\right\|_{\mathcal{H}}^{2}+C_{\mathcal{A}}\left(1+T^{*}\right) \sup _{0 \leq \tau \leq 2 T^{*}}\left\|A^{\gamma} w(\tau)\right\|_{2}^{2}
\end{aligned}
$$

Usando o mesmo procedimento acima, obtemos de (3.166) que

$$
\left\|S\left(T^{*}\right) z_{0}^{1}-S\left(T^{*}\right) z_{0}^{2}\right\|_{\mathcal{H}}^{2}+\int_{T^{*}}^{2 T^{*}}\left\|S(t) z_{0}^{1}-S(t) z_{0}^{2}\right\|_{\mathcal{H}}^{2} d t \leq\left(1+c^{-1}\right) e^{2 c T^{*}}\left\|z_{0}^{1}-z_{0}^{2}\right\|_{\mathcal{H}}^{2} .
$$

Além dos espaços acima, consideremos o subconjunto $\mathcal{A}_{T^{*}} \subset \mathcal{H}_{T^{*}}$ definido por

$$
\mathcal{A}_{T^{*}}=\left\{U:=\left(u(0), u_{t}(0), \eta^{0}, u(t)\right) \mid t \in\left[0, T^{*}\right],\left(u(0), u_{t}(0), \eta^{0}\right) \in \mathcal{A}\right\},
$$

que é fechado e limitado em $\mathcal{H}_{T^{*}}$, onde relembramos que $\mathcal{A}$ é o atrator global compacto para o semigrupo $S(t)$ e $\left(u(t), u_{t}(t), \eta^{t}\right)$ é a solução do problema (3.5)-(3.8) correspondente ao dado inicial $\left(u(0), u_{t}(0), \eta^{0}\right)$.

Também definamos o operador $\mathcal{T}: \mathcal{A}_{T^{*}} \rightarrow \mathcal{H}_{T^{*}}$ pela fórmula

$$
\mathcal{T}\left(u(0), u_{t}(0), \eta^{0}, u(t)\right)=\left(u\left(T^{*}\right), u_{t}\left(T^{*}\right), \eta^{T^{*}}, u\left(T^{*}+t\right)\right),
$$

para cada $\left(u(0), u_{t}(0), \eta^{0}, u(t)\right) \in \mathcal{A}_{T^{*}}$.

No que segue, verificaremos que o subconjunto $\mathcal{A}_{T^{*}}$ e operador $\mathcal{T}$ satisfazem as hipóteses da Proposição 1.6.6. Com efeito, devido a invariância do atrator $\mathcal{A}$ pelo semigrupo $S(t)$ (sendo que o mesmo contém todas as trajetórias a partir de $\mathcal{A}$ ), segue que $\mathcal{T}\left(\mathcal{A}_{T^{*}}\right)=\mathcal{A}_{T^{*}}$.

Além disso, afirmamos que operador $\mathcal{T}$ definido por (3.171) é Lipschitz em $\mathcal{A}_{T^{*}}$, isto é, que existe uma constante $L_{T^{*}}>0$ tal que

$$
\left\|\mathcal{T}\left(U_{1}\right)-\mathcal{T}\left(U_{2}\right)\right\|_{\mathcal{H}_{T^{*}}} \leq L_{T^{*}}\left\|U_{1}-U_{2}\right\|_{\mathcal{H}_{T^{*}}}, \quad \forall U_{1}, U_{2} \in \mathcal{A}_{T^{*}}
$$


De fato, para $i=1,2$, sejam $U_{i}=\left(u^{i}(0), u_{t}^{i}(0), \eta^{i, 0}, u^{i}(t)\right) \in \mathcal{A}_{T^{*}}$, com $t \in\left[0, T^{*}\right] \mathrm{e}$ $z^{i}(0):=\left(u^{i}(0), u_{t}^{i}(0), \eta^{i, 0}\right) \in \mathcal{A}$. Denotando por

$$
S(t) z^{i}(0):=\left(u^{i}(t), u_{t}^{i}(t), \eta^{i, t}\right) \in \mathcal{A}, \quad t \geq 0,
$$

então

$$
\left\|U_{1}-U_{2}\right\|_{\mathcal{H}_{T^{*}}}^{2}=\left\|z^{1}(0)-z^{2}(0)\right\|_{\mathcal{H}}^{2}+\int_{0}^{T^{*}}\left(\|\Delta w(t)\|_{2}^{2}+\left\|w_{t}(t)\right\|_{2}^{2}\right) d t
$$

onde denotamos $w(t)=u^{1}(t)-u^{2}(t)$ para $t \geq 0$. Logo, usando a estimativa (3.170) e (3.173), obtemos que

$$
\begin{aligned}
& \left\|\mathcal{T}\left(U_{1}\right)-\mathcal{T}\left(U_{2}\right)\right\|_{\mathcal{H}_{T^{*}}}^{2} \\
& \quad=\left\|S\left(T^{*}\right) z^{1}(0)-S\left(T^{*}\right) z^{2}(0)\right\|_{\mathcal{H}}^{2}+\int_{0}^{T^{*}}\left(\left\|\Delta w\left(t+T^{*}\right)\right\|_{2}^{2}+\left\|w_{t}\left(t+T^{*}\right)\right\|_{2}^{2}\right) d t \\
& \quad=\left\|S\left(T^{*}\right) z^{1}(0)-S\left(T^{*}\right) z^{2}(0)\right\|_{\mathcal{H}}^{2}+\int_{T^{*}}^{2 T^{*}}\left(\|\Delta w(t)\|_{2}^{2}+\left\|w_{t}(t)\right\|_{2}^{2}\right) d t \\
& \quad \leq\left\|S\left(T^{*}\right) z^{1}(0)-S\left(T^{*}\right) z^{2}(0)\right\|_{\mathcal{H}}^{2}+\int_{T^{*}}^{2 T^{*}}\left\|S(t) z^{1}(0)-S(t) z^{2}(0)\right\|_{\mathcal{H}}^{2} d t . \\
& \leq\left(1+c^{-1}\right) e^{2 c T^{*}}\left\|z^{1}(0)-z^{2}(0)\right\|_{\mathcal{H}}^{2} \\
& \leq\left(1+c^{-1}\right) e^{2 c T^{*}}\left\|U_{1}-U_{2}\right\|_{\mathcal{H}_{T^{*}}}^{2} .
\end{aligned}
$$

Isto mostra a condição (3.172) $\operatorname{com} L_{T^{*}}^{2}=\left(1+c^{-1}\right) e^{2 c T^{*}}>0$.

Resta mostrar o terceito item da Proposição 1.6.6. Primeiro, como

$$
V_{2}=D\left(A^{1 / 2}\right) \hookrightarrow \hookrightarrow D\left(A^{\gamma}\right) \hookrightarrow \hookrightarrow D\left(A^{0}\right)=V_{0},
$$

então o Lema 8 em Simon [89] implica que para qualquer $\varepsilon>0$, existe uma constante $C_{\varepsilon}>0$ tal que

$$
\left\|A^{\gamma} \omega\right\|_{2}^{2} \leq \varepsilon\|\Delta \omega\|_{2}^{2}+C_{\varepsilon}\|\omega\|_{2}^{2}, \quad \forall \omega \in V_{2} .
$$

Denotando novamente por $w(t)=u^{1}(t)-u^{2}(t)$ e $\zeta^{t}=\eta^{1, t}-\eta^{2, t}, t \geq 0$, então

$$
\begin{aligned}
\sup _{0 \leq \tau \leq 2 T^{*}}\left\|A^{\gamma} w(\tau)\right\|_{2}^{2} & \leq \varepsilon \sup _{0 \leq \tau \leq 2 T^{*}}\|\Delta w(\tau)\|_{2}^{2}+C_{\varepsilon} \sup _{0 \leq \tau \leq 2 T^{*}}\|w(\tau)\|_{2}^{2} \\
& \leq \varepsilon \sup _{0 \leq \tau \leq 2 T^{*}}\left(\|\Delta w(\tau)\|_{2}^{2}+\left\|w_{t}(\tau)\right\|_{2}^{2}+\left\|\zeta^{\tau}\right\|_{\mu, 2}^{2}\right)+C_{\varepsilon} \sup _{0 \leq \tau \leq 2 T^{*}}\|w(\tau)\|_{2}^{2} \\
& =\varepsilon \sup _{0 \leq \tau \leq 2 T^{*}}\left\|S(\tau) z_{0}^{1}-S(\tau) z_{0}^{2}\right\|_{\mathcal{H}}^{2}+C_{\varepsilon} \sup _{0 \leq \tau \leq 2 T^{*}}\|w(\tau)\|_{2}^{2} .
\end{aligned}
$$

Assim, a estimativa (3.166) infere que

$$
\sup _{0 \leq \tau \leq 2 T^{*}}\left\|A^{\gamma} w(\tau)\right\|_{2}^{2} \leq \varepsilon e^{2 c T^{*}}\left\|z_{0}^{1}-z_{0}^{2}\right\|_{\mathcal{H}}^{2}+C_{\varepsilon} \sup _{0 \leq \tau \leq 2 T^{*}}\|w(\tau)\|_{2}^{2} .
$$

Combinando (3.169) com (3.174), obtemos

$$
\begin{aligned}
\left\|S\left(T^{*}\right) z_{0}^{1}-S\left(T^{*}\right) z_{0}^{2}\right\|_{\mathcal{H}}^{2}+ & \int_{T^{*}}^{2 T^{*}}\left\|S(t) z_{0}^{1}-S(t) z_{0}^{2}\right\|_{\mathcal{H}}^{2} d t \\
& \leq q_{\varepsilon, T^{*}}^{2}\left\|z_{0}^{1}-z_{0}^{2}\right\|_{\mathcal{H}}^{2}+K_{\varepsilon, T^{*}}^{2} \sup _{0 \leq \tau \leq 2 T^{*}}\|w(\tau)\|_{2}^{2},
\end{aligned}
$$


onde as constantes $q_{\varepsilon, T^{*}}^{2}>0$ e $K_{\varepsilon, T^{*}}^{2}>0$ são dadas por

$$
q_{\varepsilon, T^{*}}^{2}=3\left(1+\nu^{-1}\right) e^{-\nu T^{*}}+\varepsilon C_{\mathcal{A}}\left(1+T^{*}\right) e^{2 c T^{*}} \quad \text { e } \quad K_{\varepsilon, T^{*}}^{2}=C_{\varepsilon} C_{\mathcal{A}}\left(1+T^{*}\right) .
$$

Aproveitando as notações anteriores, resulta da estimativa (3.175) que

$$
\left\|\mathcal{T}\left(U_{1}\right)-\mathcal{T}\left(U_{2}\right)\right\|_{\mathcal{H}_{T^{*}}}^{2} \leq q_{\varepsilon, T^{*}}^{2}\left\|U_{1}-U_{2}\right\|_{\mathcal{H}_{T^{*}}}^{2}+K_{\varepsilon, T^{*}}^{2} \sup _{0 \leq \tau \leq 2 T^{*}}\left\|u^{1}(\tau)-u^{2}(\tau)\right\|_{2}^{2},
$$

para todos $U_{i}=\left(u^{i}(0), u_{t}^{i}(0), \eta^{i, 0}, u^{i}(t)\right) \in \mathcal{A}_{T^{*}}, i=1,2$.

Desta maneira, escolhendo $T^{*}>0$ suficientemente grande, e em seguida $\varepsilon>0$ pequeno o suficiente, tal que $q:=2^{1 / 2} q_{\varepsilon, T^{*}}<1$ e $K:=2^{1 / 2} K_{\varepsilon, T^{*}}>0$, e definindo a função

$$
n_{T^{*}}(U):=\sup _{0 \leq \tau \leq T^{*}}\|w(\tau)\|_{2} \quad \text { para } \quad U=\left(u, u_{t}, \eta, w\right) \in \mathcal{H}_{T^{*}},
$$

concluimos de (3.176) que

$$
\left\|\mathcal{T}\left(U_{1}\right)-\mathcal{T}\left(U_{2}\right)\right\|_{\mathcal{H}_{T^{*}}} \leq q\left\|U_{1}-U_{2}\right\|_{\mathcal{H}_{T^{*}}}+K\left[n\left(U_{1}-U_{2}\right)+n\left(T\left(U_{1}\right)-T\left(U_{2}\right)\right)\right],
$$

para todos $U_{1}, U_{2} \in \mathcal{A}_{T^{*}}$. Agora note que a função $n_{T^{*}}(U)$ definida em (3.177) é uma seminorma compacta (segundo a Definição 1.6.10), devido a inclusão compacta de $W_{1}\left(0, T^{*}\right)$ em $C\left(0, T^{*} ; V_{0}\right)$, pelo Corolário 4 em Simon [89] (ver também Chueshov \& Lasiecka [26]).

Portanto, o subconjunto $\mathcal{A}_{T^{*}}$ e operador $\mathcal{T}$ satisfazem as condições $(i)$-(iii) da Proposição 1.6.6, de onde segue que $\mathcal{A}_{T^{*}}$ é um conjunto compacto em $\mathcal{H}_{T^{*}}$ com dimensão fractal finita, isto é,

$$
\operatorname{dim}_{f} \mathcal{A}_{T^{*}}<\infty
$$

com estimativa dada por (1.13).

Para concluir que o atrator global $\mathcal{A}$ possui dimensão fractal finita, definimos o operador projeção $\mathcal{P}: \mathcal{H}_{T^{*}} \rightarrow \mathcal{H}$ por

$$
\mathcal{P}\left(u, u_{t}, \eta, w\right)=\left(u, u_{t}, \eta\right), \quad \forall\left(u, u_{t}, \eta, w\right) \in \mathcal{H}_{T^{*}}
$$

Logo, $\mathcal{P}$ é claramente Lipschitz contínuo e $\mathcal{P}\left(\mathcal{A}_{T^{*}}\right)=\mathcal{A}$. Portanto, o Lema 1.6.7 assegura que

$$
\operatorname{dim}_{f} \mathcal{A}=\operatorname{dim}_{f} \mathcal{P}\left(\mathcal{A}_{T^{*}}\right) \leq \operatorname{dim}_{f} \mathcal{A}_{T^{*}},
$$

ou seja, o atrator global compacto $\mathcal{A}$ possui dimensão fractal finita.

Isto encerra a prova Teorema 3.6.2. 


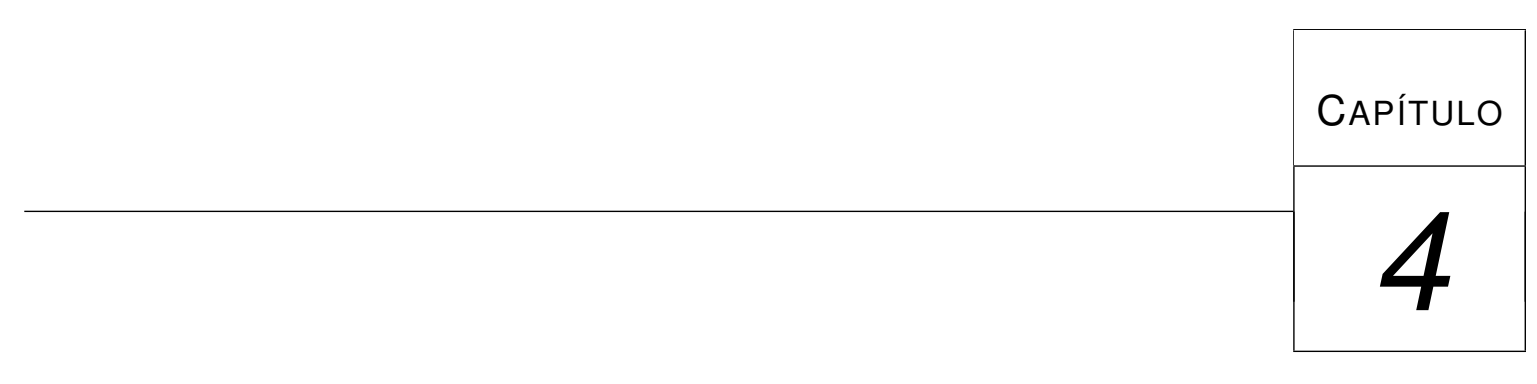

\section{Um modelo de placas de Mindlin-Timoshenko}

\subsection{Introdução}

Neste capítulo vamos estudar uma variante da equação de placas finas dada pelas equações de Mindlin-Timoshenko. Neste caso abordaremos um sistema totalmente e parcialmente dissipativo, com objetivo de obter taxas de decaimento conforme a presença das dissipações ${ }^{1}$.

Seja $\Omega$ um domínio limitado de $\mathbb{R}^{2}$ com fronteira suave $\Gamma=\partial \Omega$ e $\mathbb{R}^{+}=(0, \infty)$. Para simplificar a notação, denotemos por $\mathcal{L}_{1}, \mathcal{L}_{2}$ e $\mathcal{L}_{3}$ os seguintes operadores lineares elípticos de segunda ordem

$$
\begin{aligned}
& \mathcal{L}_{1}(\psi, \varphi, w)=\frac{\partial}{\partial x}\left(\psi+\frac{\partial w}{\partial x}\right)+\frac{\partial}{\partial y}\left(\varphi+\frac{\partial w}{\partial y}\right), \\
& \mathcal{L}_{2}(\psi, \varphi)=\frac{\partial^{2} \psi}{\partial x^{2}}+\frac{1-\mu}{2} \frac{\partial^{2} \psi}{\partial y^{2}}+\frac{1+\mu}{2} \frac{\partial^{2} \varphi}{\partial x \partial y}, \\
& \mathcal{L}_{3}(\varphi, \psi)=\frac{\partial^{2} \varphi}{\partial y^{2}}+\frac{1-\mu}{2} \frac{\partial^{2} \varphi}{\partial x^{2}}+\frac{1+\mu}{2} \frac{\partial^{2} \psi}{\partial x \partial y}
\end{aligned}
$$

para $(x, y) \in \Omega$ e $t \geq 0$, onde as fuções $\psi=\psi(x, y, t)$ e $\varphi=\varphi(x, y, t)$ representam os ângulos de rotação de um filamento da placa e a função $w=w(x, y, t)$ simboliza o deslocamento transversal da superfície média da placa. A constante $0<\mu<1 / 2$ é chamada coeficiente de Poisson, sendo proveniente de considerações físicas sobre a placa.

\footnotetext{
${ }^{1}$ Trabalho realizado em conjunto com o Professor Dr. Jaime E. Muñoz Rivera do Laboratório Nacional de Computação Científica - LNCC, Petrópolis - RJ, Brasil.
} 
No que segue, estudaremos o comportamento assintótico de soluções globais para o seguinte sistema de placas de Mindlin-Timoshenko com dissipação viscosa

$$
\begin{aligned}
& \rho h w_{t t}-K \mathcal{L}_{1}(\psi, \varphi, w)-D_{0} \Delta w_{t}=0 \\
& \frac{\rho h^{3}}{12} \psi_{t t}-D \mathcal{L}_{2}(\psi, \varphi)+K\left(\psi+\frac{\partial w}{\partial x}\right)-D_{1} \mathcal{L}_{2}\left(\psi_{t}, \varphi_{t}\right)=0 \\
& \frac{\rho h^{3}}{12} \varphi_{t t}-D \mathcal{L}_{3}(\varphi, \psi)+K\left(\varphi+\frac{\partial w}{\partial y}\right)-D_{1} \mathcal{L}_{3}\left(\varphi_{t}, \psi_{t}\right)=0
\end{aligned}
$$

em $\Omega \times \mathbb{R}^{+}$, onde, fisicamente, as constantes positivas $\rho$ representa a massa por unidade de volume, $h$ denota a espessura uniforme da placa, $K$ exprime o módulo de cisalhamento e $D$ simboliza o módulo de rigidez à flexão. Além disso, as constantes $D_{0}$ e $D_{1}$ são não negativas e as chamaremos de constantes de dissipação.

Para maiores detalhes sobre a modelagem do problema, bem como sobre as considerações físicas para tal, ver Lagnese [64] e Lagnese \& Lions [65].

Associado ao sistema (4.4)-(4.6), consideremos as condições iniciais

$$
\begin{array}{llll}
w(x, y, 0)=w_{0}(x, y), & w_{t}(x, y, 0)=w_{1}(x, y) & \text { em } & \Omega \\
\psi(x, y, 0)=\psi_{0}(x, y), & \psi_{t}(x, y, 0)=\psi_{1}(x, y) & \text { em } & \Omega \\
\varphi(x, y, 0)=\varphi_{0}(x, y), & \varphi_{t}(x, y, 0)=\varphi_{1}(x, y) & \text { em } & \Omega
\end{array}
$$

e as seguintes condições de fronteira: De Dirichlet

$$
w=\psi=\varphi=0 \quad \text { sobre } \quad \Gamma \times[0, \infty)
$$

e mistas

$$
\begin{array}{cccc}
w=0 & \text { sobre } & \Gamma \times[0, \infty), \\
\psi=0,\left(\frac{1-\mu}{2}\left(\frac{\partial \varphi}{\partial x}+\frac{\partial \psi}{\partial y}\right), \frac{\partial \varphi}{\partial y}+\mu \frac{\partial \psi}{\partial x}\right) \cdot \nu=0 & \text { sobre } & \Gamma_{1} \times[0, \infty), \\
\varphi=0,\left(\frac{\partial \psi}{\partial x}+\mu \frac{\partial \varphi}{\partial y}, \frac{1-\mu}{2}\left(\frac{\partial \varphi}{\partial x}+\frac{\partial \psi}{\partial y}\right)\right) \cdot \nu=0 & \text { sobre } & \Gamma_{2} \times[0, \infty),
\end{array}
$$

onde nas condições mistas consideramos também que a fronteira suave $\Gamma=\bar{\Gamma}_{1} \cup \bar{\Gamma}_{2} \operatorname{com} \Gamma_{1}, \Gamma_{2} \neq \emptyset$, $\Gamma_{1} \cap \Gamma_{2}=\emptyset \mathrm{e} \nu:=\left(\nu_{1}, \nu_{2}\right)$ é o vetor normal unitário exterior a $\Gamma$.

Este capítulo está organizado da seguinte maneira. Na seção 4.2 fixamos as notações preliminares e apresentaremos o resultado de existência via teoria de semigrupos lineares. Na seção 4.3 mostraremos o resultado que concede analiticidade do semigrupo associado ao sistema de MindlinTimoshenko e, em particular, obtemos estabilidade exponencial do sistema quando o mesmo é totalmente dissipativo. Na seção 4.4 discutimos a falta de decaimento exponencial para o sistema de Mindlin-Timoshenko parcialmente dissipativo. Finalmente, na seção 4.5 determinaremos um resultado de estabilidade polinomial para o sistema de Mindlin-Timoshenko parcialmente dissipativo. Além disso, mostraremos que a taxa de decaimento polinomial não pode ser melhorada quando os dados iniciais são fixados. 


\subsection{Existência e unicidade}

Inicialmente vamos reescrever o problema de valor inicial e de fronteira (4.4)-(4.9) como um problema de Cauchy abstrato de primeira ordem. Em seguida, usaremos a teoria de semigrupo recordada na seção 1.5 do Capítulo 1 para determinar um resultado de existência de unicidade de soluções globais.

Denotando por $U$ a função a valores vetoriais $U=(w, W, \psi, \Psi, \varphi, \Phi)^{t}$, onde $W, \Psi$ e $\Phi$ representam $w_{t}, \psi_{t}$ e $\varphi_{t}$, respectivamente, provaremos em seguida que $U$ é uma solução do seguinte problema

$$
U_{t}=\mathcal{A} U, \quad U(0)=U_{0},
$$

onde $U_{0}=\left(w_{0}, w_{1}, \psi_{0}, \psi_{1}, \varphi_{0}, \varphi_{1}\right)^{t}$ e o operador matricial $\mathcal{A}$ é dado por

$$
\mathcal{A}=\left(\begin{array}{cccccc}
0 & I_{d} & 0 & 0 & 0 & 0 \\
\frac{K}{\rho_{1}} \Delta & \frac{D_{0}}{\rho_{1}} \Delta & \frac{K}{\rho_{1}} \partial_{x} & 0 & \frac{K}{\rho_{1}} \partial_{y} & 0 \\
0 & 0 & 0 & I_{d} & 0 & 0 \\
-\frac{K}{\rho_{2}} \partial_{x} & 0 & \frac{1}{\rho_{2}}\left(D \mathcal{B}_{1}-K I_{d}\right) & \frac{D_{1}}{\rho_{2}} \mathcal{B}_{1} & \frac{D}{\rho_{2}} \mathcal{B}_{2} & \frac{D_{1}}{\rho_{2}} \mathcal{B}_{2} \\
0 & 0 & 0 & 0 & 0 & I_{d} \\
-\frac{K}{\rho_{2}} \partial_{y} & 0 & \frac{D}{\rho_{2}} \mathcal{B}_{2} & \frac{D_{1}}{\rho_{2}} \mathcal{B}_{2} & \frac{1}{\rho_{2}}\left(D \mathcal{B}_{3}-K I_{d}\right) & \frac{D_{1}}{\rho_{2}} \mathcal{B}_{3}
\end{array}\right),
$$

onde denotamos por $\rho_{1}:=\rho h$ e $\rho_{2}:=\frac{\rho h^{3}}{12}$, e também por $\mathcal{B}_{i}, i=1,2,3$, os seguintes operadores diferenciais

$$
\begin{aligned}
\mathcal{B}_{1} & :=\partial_{x}^{2}+\frac{1-\mu}{2} \partial_{y}^{2}, \\
\mathcal{B}_{2} & :=\frac{1+\mu}{2} \partial_{x y}^{2}, \\
\mathcal{B}_{3} & :=\partial_{y}^{2}+\frac{1-\mu}{2} \partial_{x}^{2} .
\end{aligned}
$$

Observação 4.2.1. Nas considerações a seguir é conveniente ter em mente a seguinte expressão

$$
\mathcal{A} U=\left[\begin{array}{c}
W \\
\frac{K}{\rho_{1}} \mathcal{L}_{1}(\psi, \varphi, w)+\frac{D_{0}}{\rho_{1}} \Delta W \\
\Psi \\
\frac{D}{\rho_{2}} \mathcal{L}_{2}(\psi, \varphi)-\frac{K}{\rho_{2}}\left(\psi+w_{x}\right)+\frac{D_{1}}{\rho_{2}} \mathcal{L}_{2}(\Psi, \Phi) \\
\Phi \\
\frac{D}{\rho_{2}} \mathcal{L}_{3}(\varphi, \psi)-\frac{K}{\rho_{2}}\left(\varphi+w_{y}\right)+\frac{D_{1}}{\rho_{2}} \mathcal{L}_{3}(\Phi, \Psi)
\end{array}\right] \text { para } U=\left[\begin{array}{c}
w \\
W \\
\psi \\
\Psi \\
\varphi \\
\Phi
\end{array}\right]
$$

Para atender ambas as condições de fronteira acopladas ao sistema de Mindlin-Timoshenko consideremos os seguinte espaços

$$
\mathcal{H}_{1}=H_{0}^{1}(\Omega) \times L^{2}(\Omega) \times H_{0}^{1}(\Omega) \times L^{2}(\Omega) \times H_{0}^{1}(\Omega) \times L^{2}(\Omega)
$$

e

$$
\mathcal{H}_{2}=H_{0}^{1}(\Omega) \times L^{2}(\Omega) \times H_{\Gamma_{1}}^{1}(\Omega) \times L^{2}(\Omega) \times H_{\Gamma_{2}}^{1}(\Omega) \times L^{2}(\Omega),
$$


onde

$$
\begin{aligned}
& H_{\Gamma_{1}}^{1}(\Omega)=\left\{\psi \in H^{1}(\Omega) ; \psi=\mu \psi_{x} \nu_{2}+\frac{1-\mu}{2} \psi_{y} \nu_{1}=0 \mathrm{em} \Gamma_{1}, \psi_{x} \nu_{1}+\frac{1-\mu}{2} \psi_{y} \nu_{2}=0 \mathrm{em} \Gamma_{2}\right\}, \\
& H_{\Gamma_{2}}^{1}(\Omega)=\left\{\varphi \in H^{1}(\Omega) ; \varphi=\frac{1-\mu}{2} \varphi_{x} \nu_{2}+\mu \varphi_{y} \nu_{1}=0 \mathrm{em} \Gamma_{2}, \frac{1-\mu}{2} \varphi_{x} \nu_{1}+\varphi_{y} \nu_{2}=0 \mathrm{em} \Gamma_{1}\right\} .
\end{aligned}
$$

Em cada caso o domínio do operador $\mathcal{A}$ é dado por

$$
\begin{aligned}
\mathcal{D}\left(\mathcal{A}_{1}\right)=\left\{U \in \mathcal{H}_{1} ;\right. & W, \Psi, \Phi \in H_{0}^{1}(\Omega), K \mathcal{L}_{1}(\psi, \varphi, w)+D_{0} \Delta W \\
& \left.D \mathcal{L}_{2}(\psi, \varphi)+D_{1} \mathcal{L}_{2}(\Psi, \Phi), D \mathcal{L}_{3}(\varphi, \psi)+D_{1} \mathcal{L}_{3}(\Phi, \Psi) \in L^{2}(\Omega)\right\}
\end{aligned}
$$

$\mathrm{e}$

$$
\begin{array}{r}
\mathcal{D}\left(\mathcal{A}_{2}\right)=\left\{U \in \mathcal{H}_{2} ; W \in H_{0}^{1}(\Omega), \Psi \in H_{\Gamma_{1}}^{1}(\Omega), \Phi \in H_{\Gamma_{2}}^{1}(\Omega), K \mathcal{L}_{1}(\psi, \varphi, w)+D_{0} \Delta W\right. \\
\left.D \mathcal{L}_{2}(\psi, \varphi)+D_{1} \mathcal{L}_{2}(\Psi, \Phi), D \mathcal{L}_{3}(\varphi, \psi)+D_{1} \mathcal{L}_{3}(\Phi, \Psi) \in L^{2}(\Omega)\right\} .
\end{array}
$$

Quando as condições de fronteira não influenciar nas definições acima (não havendo possibilidade de confusão), denotaremos $\mathcal{H}_{i}$ e $\mathcal{D}\left(\mathcal{A}_{i}\right)$ simplesmente por $\mathcal{H}$ e $\mathcal{D}(\mathcal{A})$, respectivamente.

Como é usual, denotaremos também neste capítulo por $(\cdot, \cdot)$ o produto interno em $L^{2}(\Omega)$ e por $\|\cdot\|_{p}$ a norma em $L^{p}(\Omega)$. Devido a "simetria" do problema de Mindlin-Timoshenko usaremos a seguinte norma em $\mathcal{H}$

$$
\begin{aligned}
\|U\|_{\mathcal{H}}^{2}= & \rho_{1}\|W\|_{2}^{2}+\rho_{2}\|\Psi\|_{2}^{2}+\rho_{2}\|\Phi\|_{2}^{2}+K\left\|\psi+w_{x}\right\|_{2}^{2}+K\left\|\varphi+w_{y}\right\|_{2}^{2} \\
& +D\left\|\psi_{x}\right\|_{2}^{2}+D\left\|\varphi_{y}\right\|_{2}^{2}+D\left(\frac{1-\mu}{2}\right)\left\|\psi_{y}+\varphi_{x}\right\|_{2}^{2}+2 D \mu \operatorname{Re}\left(\psi_{x}, \varphi_{y}\right),
\end{aligned}
$$

que é proveniente do produto interno em $\mathcal{H}$ dado por

$$
\begin{aligned}
(U, \hat{U})_{\mathcal{H}}= & \rho_{1}(W, \hat{W})+\rho_{2}(\Psi, \hat{\Psi})+\rho_{2}(\Phi, \hat{\Phi})+K\left(\psi+w_{x}, \hat{\psi}+\hat{w}_{x}\right)+K\left(\varphi+w_{y}, \hat{\varphi}+\hat{w}_{y}\right) \\
& +D\left[\left(\psi_{x}, \hat{\psi}_{x}\right)+\left(\varphi_{y}, \hat{\varphi}_{y}\right)+\left(\frac{1-\mu}{2}\right)\left(\psi_{y}+\varphi_{x}, \hat{\psi}_{y}+\hat{\varphi}_{x}\right)+\mu\left(\psi_{x}, \hat{\varphi}_{y}\right)+\mu\left(\varphi_{y}, \hat{\psi}_{x}\right)\right],
\end{aligned}
$$

para $U=(w, W, \psi, \Psi, \varphi, \Phi)^{t}, \hat{U}=(\hat{w}, \hat{W}, \hat{\psi}, \hat{\Psi}, \hat{\varphi}, \hat{\Phi})^{t} \in \mathcal{H}$. Com isto, $\left(\mathcal{H},\|\cdot\|_{\mathcal{H}},(\cdot, \cdot)_{\mathcal{H}}\right)$ é um espaço de Hilbert. Por conveniência, notamos que a norma em (4.12) pode ser reescrita sob uma forma não negativa como

$$
\begin{aligned}
\|U\|_{\mathcal{H}}^{2}= & \rho_{1}\|W\|_{2}^{2}+\rho_{2}\|\Psi\|_{2}^{2}+\rho_{2}\|\Phi\|_{2}^{2}+K\left\|\psi+w_{x}\right\|_{2}^{2}+K\left\|\varphi+w_{y}\right\|_{2}^{2} \\
& +D(1-\mu)\left[\left\|\psi_{x}\right\|_{2}^{2}+\left\|\varphi_{y}\right\|_{2}^{2}\right]+D\left(\frac{1-\mu}{2}\right)\left\|\psi_{y}+\varphi_{x}\right\|_{2}^{2}+D \mu\left\|\psi_{x}+\varphi_{y}\right\|_{2}^{2} .
\end{aligned}
$$

Observação 4.2.2. Um resultado que fornece a equivalência da norma acima em (4.12) com a norma usual em $\mathcal{H}$ é dada pelo Lema 2.1 em Lagnese [64, Capítulo 3]. Além disso, este resultado será importante para obter a existência e a estabilidade assintótica de soluções. Por comodidade, apresentaremos uma versão de tal lema usando notações apropriadas a fim de usá-lo em considerações futuras. 
Neste contexto, denotaremos por $\mathcal{V}$ e $\mathcal{M}$ os espaços

$$
\mathcal{V}:=H_{0}^{1}(\Omega) \times H_{0}^{1}(\Omega) \quad\left(\text { ou } H_{\Gamma_{1}}^{1}(\Omega) \times H_{\Gamma_{2}}^{1}(\Omega)\right)
$$

$\mathrm{e}$

$$
\mathcal{M}:=H_{0}^{1}(\Omega) \times H_{0}^{1}(\Omega) \times H_{0}^{1}(\Omega) \quad\left(\text { ou } H_{0}^{1}(\Omega) \times H_{\Gamma_{1}}^{1}(\Omega) \times H_{\Gamma_{2}}^{1}(\Omega)\right) .
$$

Como em Lagnese [64, Capítulo 3] o seguinte lema é uma consequência da desigualdade de Korn.

Lema 4.2.1. Sob as considerações acima, temos:

(a) Existe uma constante $\alpha_{0}>0$ tal que, para todo par $(\psi, \varphi) \in \mathcal{V}$,

$$
\begin{aligned}
\alpha_{0}\left[\|\psi\|_{H^{1}(\Omega)}^{2}+\|\varphi\|_{H^{1}(\Omega)}^{2}\right] \leq & D\left\|\psi_{x}\right\|_{2}^{2}+D\left\|\varphi_{y}\right\|_{2}^{2} \\
& +D\left(\frac{1-\mu}{2}\right)\left\|\psi_{y}+\varphi_{x}\right\|_{2}^{2}+2 D \mu \operatorname{Re}\left(\psi_{x}, \varphi_{y}\right) .
\end{aligned}
$$

(b) Para cada $K_{0}>0$, existe $\alpha\left(K_{0}\right)>0$ tal que, para todo $(w, \psi, \varphi) \in \mathcal{M}$ e $K \geq K_{0}$,

$$
\begin{aligned}
\alpha\left(K_{0}\right)\|(w, \psi, \varphi)\|_{\mathcal{M}}^{2} \leq & K\left\|\psi+w_{x}\right\|_{2}^{2}+K\left\|\varphi+w_{y}\right\|_{2}^{2}+D\left\|\psi_{x}\right\|_{2}^{2} \\
& +D\left\|\varphi_{y}\right\|_{2}^{2}+D\left(\frac{1-\mu}{2}\right)\left\|\psi_{y}+\varphi_{x}\right\|_{2}^{2}+2 D \mu \operatorname{Re}\left(\psi_{x}, \varphi_{y}\right) .
\end{aligned}
$$

Com relação ao domínio do operador $\mathcal{A}$, podemos considerar ainda os espaços (de Hilbert) mais regulares

$$
\mathcal{D}\left(\mathcal{A}^{k}\right)=\left\{U \in \mathcal{D}\left(\mathcal{A}^{k-1}\right) ; \mathcal{A} U \in \mathcal{D}\left(\mathcal{A}^{k-1}\right)\right\}, \quad k \in \mathbb{N}
$$

equipados com a norma $\|U\|_{\mathcal{D}\left(\mathcal{A}^{k}\right)}^{2}=\sum_{j=0}^{k}\|U\|_{\mathcal{D}\left(\mathcal{A}^{j}\right)}^{2}$, ver seção 1.4 para mais detalhes.

A existência e unicidade de solução para o problema (4.10) está resumida no resultado subsequente.

Teorema 4.2.2. Se $U_{0}=\left(w_{0}, w_{1}, \psi_{0}, \psi_{1}, \varphi_{0}, \varphi_{1}\right)^{t} \in \mathcal{D}\left(\mathcal{A}_{i}\right)$, então existe uma única solução $U=(w, W, \psi, \Psi, \varphi, \Phi)^{t}$ para o problema (4.10) satisfazendo

$$
U \in C\left([0,+\infty), \mathcal{D}\left(\mathcal{A}_{i}\right)\right) \cap C^{1}\left([0,+\infty), \mathcal{H}_{i}\right), \quad i=1,2 .
$$

Além disso, se $U_{0} \in \mathcal{D}\left(\mathcal{A}^{k}\right), k \in \mathbb{N}$, então a solução $U$ do problema (4.10) satisfaz.

$$
U \in \bigcap_{j=0}^{k} C^{k-j}\left([0,+\infty), \mathcal{D}\left(\mathcal{A}^{j}\right)\right), \quad i=1,2 .
$$

Prova. Procedendo da mesma maneira como na obra de Lagnese [64, Capítulo 3] obtemos que o operador $\mathcal{A}$ definido por (4.11) é dissipativo em $\mathcal{H}$, densamente definido e que $0 \in \rho(\mathcal{A})$. Isto acarretaria, pelo Corolário 1.5.3, que $\mathcal{A}$ é gerador infinitesimal de um $C_{0}$-semigrupo de contrações em $\mathcal{H}$. Logo, utilizando os Teoremas 1.5.7 e 1.5.8, segue a existência e unicidade de solução para o problema (4.10), o que conclui a tese do Teorema 4.2.2. 
No que segue, afim de tornar o texto um pouco mais dinâmico, apresentaremos a ideia para obter a dissipatividade de $\mathcal{A}$, isto é, que

$$
\operatorname{Re}(\mathcal{A} U, U)_{\mathcal{H}} \leq 0, \quad \forall U \in \mathcal{D}(\mathcal{A})
$$

que $\mathcal{D}(\mathcal{A})$ é denso em $\mathcal{H}$ e também que $0 \in \rho(\mathcal{A})$. Com efeito, em primeiro lugar, usando a fórmula de Green notamos que

$$
\begin{aligned}
(\mathcal{A} U, U)_{\mathcal{H}}= & -K\left[\left(\psi+w_{x}, \Psi+W_{x}\right)-\overline{\left(\psi+w_{x}, \Psi+W_{x}\right)}\right] \\
& -K\left[\left(\varphi+w_{y}, \Phi+W_{y}\right)-\overline{\left(\varphi+w_{y}, \Phi+W_{y}\right)}\right] \\
& -D\left[\left(\psi_{x}, \Psi_{x}\right)-\overline{\left(\psi_{x}, \Psi_{x}\right)}\right]-D\left[\left(\varphi_{y}, \Phi_{y}\right)-\overline{\left(\varphi_{y}, \Phi_{y}\right)}\right] \\
& -D\left(\frac{1-\mu}{2}\right)\left[\left(\varphi_{x}+\psi_{y}, \Phi_{x}+\Psi_{y}\right)-\overline{\left(\varphi_{x}+\psi_{y}, \Phi_{x}+\Psi_{y}\right)}\right] \\
& -D \mu\left[\left(\psi_{x}, \Phi_{y}\right)-\overline{\left(\psi_{x}, \Phi_{y}\right)}\right]-D \mu\left[\left(\varphi_{y}, \Psi_{x}\right)-\overline{\left(\varphi_{y}, \Psi_{x}\right)}\right] \\
& -D_{0}\|\nabla W\|_{2}^{2}-D_{1}(1-\mu)\left\|\Psi_{x}\right\|_{2}^{2}-D_{1}(1-\mu)\left\|\Phi_{y}\right\|_{2}^{2} \\
& -D_{1} \mu\left\|\Psi_{x}+\Phi_{y}\right\|_{2}^{2}-D_{1}\left(\frac{1-\mu}{2}\right)\left\|\Psi_{y}+\Phi_{x}\right\|_{2}^{2} \\
& +K \int_{\Gamma} \bar{W}\left(\psi+w_{x}, \varphi+w_{y}\right) \cdot \nu d \Gamma+D_{0} \int_{\Gamma} \bar{W}(\nabla W \cdot \nu) d \Gamma \\
& +D \int_{\Gamma} \bar{\Psi}\left(\psi_{x}+\mu \varphi_{y}, \frac{1-\mu}{2}\left(\varphi_{x}+\psi_{y}\right)\right) \cdot \nu d \Gamma \\
& +D_{1} \int_{\Gamma} \bar{\Psi}\left(\Psi_{x}+\mu \Phi_{y}, \frac{1-\mu}{2}\left(\Phi_{x}+\Psi_{y}\right)\right) \cdot \nu d \Gamma \\
& +D \int_{\Gamma} \bar{\Phi}\left(\frac{1-\mu}{2}\left(\varphi_{x}+\psi_{y}\right), \varphi_{y}+\mu \psi_{x}\right) \cdot \nu d \Gamma \\
& +D_{1} \int_{\Gamma} \bar{\Phi}\left(\frac{1-\mu}{2}\left(\Phi_{x}+\Psi_{y}\right), \Phi_{y}+\mu \Psi_{x}\right) \cdot \nu d \Gamma .
\end{aligned}
$$

Logo, para $U \in \mathcal{D}(\mathcal{A})$ obtemos que as integrais sobre a fronteira $\Gamma$ que surgem na equação acima se anulam (em ambos os casos com respeito as condições de fronteira). Então, tomando a parte real concluimos que

$$
\begin{aligned}
\operatorname{Re}(\mathcal{A} U, U)_{\mathcal{H}}= & -D_{0}\|\nabla W\|_{2}^{2}-D_{1}(1-\mu)\left\|\Psi_{x}\right\|_{2}^{2}-D_{1}(1-\mu)\left\|\Phi_{y}\right\|_{2}^{2} \\
& -D_{1}\left(\frac{1-\mu}{2}\right)\left\|\Phi_{x}+\Psi_{y}\right\|_{2}^{2}-D_{1} \mu\left\|\Psi_{x}+\Phi_{y}\right\|_{2}^{2},
\end{aligned}
$$

para todo $U \in \mathcal{D}(\mathcal{A})$. Como $D_{0}, D_{1} \geq 0$, segue que o operador $\mathcal{A}$ é dissipativo em $\mathcal{H}$.

Agora notamos que $0 \in \rho(\mathcal{A})$, ou seja, $-\mathcal{A}$ é invertível. Isto segue do fato que a equação resolvente $-\mathcal{A} U=F$ possui uma única solução $U \in \mathcal{D}(\mathcal{A})$, qualquer que seja $F \in \mathcal{H}$ dado. De fato, pondo $F=\left(f^{1}, f^{2}, f^{3}, f^{4}, f^{5}, f^{6}\right)^{t} \in \mathcal{H}$ e escrevendo a equação resolvente em termos de 
suas componentes, obtemos

$$
\begin{aligned}
-W & =f^{1} \\
-K\left[\left(\psi+w_{x}\right)_{x}+\left(\varphi+w_{y}\right)_{y}\right]-D_{0} \Delta W & =\rho_{1} f^{2} \\
-\Psi & =f^{3} \\
-D \mathcal{L}_{2}(\psi, \varphi)+K\left(\psi+w_{x}\right)-D_{1} \mathcal{L}_{2}(\Psi, \Phi) & =\rho_{2} f^{4} \\
-\Phi & =f^{5} \\
-D \mathcal{L}_{3}(\varphi, \psi)+K\left(\varphi+w_{y}\right)-D_{1} \mathcal{L}_{3}(\Phi, \Psi) & =\rho_{2} f^{6} .
\end{aligned}
$$

Logo, consideramos

$$
W=-f^{1}, \quad \Psi=-f^{3} \quad \text { e } \quad \Phi=-f^{5},
$$

de onde resulta que

$$
\begin{aligned}
-K\left(\psi+w_{x}\right)_{x}-K\left(\varphi+w_{y}\right)_{y} & =-D_{0} \Delta f^{1}+\rho_{1} f^{2} \\
-D \mathcal{L}_{2}(\psi, \varphi)+K\left(\psi+w_{x}\right) & =-D_{1} \mathcal{L}_{2}\left(f^{3}, f^{5}\right)+\rho_{2} f^{4} \\
-D \mathcal{L}_{3}(\varphi, \psi)+K\left(\varphi+w_{y}\right) & =-D_{1} \mathcal{L}_{3}\left(f^{5}, f^{3}\right)+\rho_{2} f^{6}
\end{aligned}
$$

Como consequência do Teorema de Lax-Milgram e de que a forma bilinear $a(\cdot, \cdot)$ definida por

$$
\begin{aligned}
a((w, \psi, \varphi),(\hat{w}, \hat{\psi}, \hat{\varphi}))= & K\left(\psi+w_{x}, \hat{\psi}+\hat{w}_{x}\right)+K\left(\varphi+w_{y}, \hat{\varphi}+\hat{w}_{y}\right)+D\left(\psi_{x}, \hat{\psi}_{x}\right)+D\left(\varphi_{y}, \hat{\varphi}_{y}\right) \\
& +D\left(\frac{1-\mu}{2}\right)\left(\psi_{y}+\varphi_{x}, \hat{\psi}_{y}+\hat{\varphi}_{x}\right)+D \mu\left(\psi_{x}, \hat{\varphi}_{y}\right)+D \mu\left(\varphi_{y}, \hat{\psi}_{x}\right),
\end{aligned}
$$

é contínua e coerciva em

$$
H_{0}^{1}(\Omega) \times H_{0}^{1}(\Omega) \times H_{0}^{1}(\Omega) \quad \text { e } \quad H_{0}^{1}(\Omega) \times H_{\Gamma_{1}}^{1}(\Omega) \times H_{\Gamma_{2}}^{1}(\Omega),
$$

segue que o sistema acima possui uma única solução $(w, \psi, \varphi)$ nos espaços desejados (ver os detalhes em Lagnese [64, Capítulo 3]).

Por fim, uma vez que o operador $\mathcal{A}$ é dissipativo e que $0 \in \rho(\mathcal{A})$, usamos os Teoremas 4.5 e 4.6 em Pazy [85, Capítulo 1] para concluir que $\overline{\mathcal{D}(\mathcal{A})}=\mathcal{H}$.

Observação 4.2.3. Os detalhes na prova de existência foram omitidos, pois eles não representam diferenças adicionais com relação aos trabalhos mencionados anteriormente, referentes à equação de placas de Mindlin-Timoshenko. Contudo, a partir da próxima de seção deste capítulo os resultados são mais específicos deste trabalho e, por este motivo, os apresentaremos mais detalhadamente.

Nas seções seguintes vamos estudar o comportamento assintótico das soluções de (4.10) por meio do semigrupo linear $S(t):=e^{\mathcal{A} t}, t \geq 0$, dado por

$$
\begin{aligned}
e^{\mathcal{A} t}: \mathcal{H} & \longrightarrow \mathcal{H} \\
U_{0} & \longmapsto e^{\mathcal{A} t} U_{0}=U(t),
\end{aligned}
$$

o qual chamaremos de $C_{0}$-semigrupo de contrações associado ao sistema de Mindlin-Timoshenko. 


\subsection{Analiticidade}

Nesta seção vamos mostrar que o $C_{0}$-semigrupo de contrações $S(t)=e^{\mathcal{A} t}$ associado ao sistema de Mindlin-Timoshenko dissipativo é analítico quando consideramos $D_{0}, D_{1}>0$. Como consequência, concluiremos também que $S(t)$ é exponencialmente estável, o que nos fornece estabilidade exponencial de soluções. Para concluir tais objetivos, faremos uso dos Teoremas 1.5.4 e 1.5.5 introduzidos na seção 1.5 do Capítulo 1. Em resumo, o principal resultado desta seção é o seguinte:

Teorema 4.3.1. Sob as condições do Teorema 4.2.2, se $D_{0}, D_{1}>0$, então o $C_{0}$-semigrupo de contrações $S(t)=e^{\mathcal{A} t}$ associado ao sistema de Mindlin-Timoshenko é analítico.

Prova. De acordo com o Teorema 1.5.4 devemos mostrar que $S(t)=e^{\mathcal{A} t}$ satisfaz as seguintes condições:

$$
i \mathbb{R} \subset \rho(\mathcal{A})
$$

$\mathrm{e}$

$$
\overline{\lim }_{|\beta| \rightarrow \infty}\left\|\beta\left(i \beta I_{d}-A\right)^{-1}\right\|<\infty .
$$

Nosso ponto de partida é considerar a equação resolvente

$$
i \beta U-\mathcal{A} U=F \text { em } \mathcal{H},
$$

que para $F=\left(f^{1}, f^{2}, f^{3}, f^{4}, f^{5}, f^{6}\right)^{t} \in \mathcal{H}$ e $U=(w, W, \psi, \Psi, \varphi, \Phi)^{t} \in \mathcal{D}(\mathcal{A})$, pode ser reescrita em termos de suas componente como segue

$$
\begin{aligned}
i \beta w-W & =f^{1} \\
i \beta W-\frac{K}{\rho_{1}}\left[\left(\psi+w_{x}\right)_{x}+\left(\varphi+w_{y}\right)_{y}\right]-\frac{D_{0}}{\rho_{1}} \Delta W & =f^{2} \\
i \beta \psi-\Psi & =f^{3} \\
i \beta \Psi-\frac{D}{\rho_{2}} \mathcal{L}_{2}(\psi, \varphi)+\frac{K}{\rho_{2}}\left(\psi+w_{x}\right)-\frac{D_{1}}{\rho_{2}} \mathcal{L}_{2}(\Psi, \Phi) & =f^{4} \\
i \beta \varphi-\Phi & =f^{5} \\
i \beta \Phi-\frac{D}{\rho_{2}} \mathcal{L}_{3}(\varphi, \psi)+\frac{K}{\rho_{2}}\left(\varphi+w_{y}\right)-\frac{D_{1}}{\rho_{2}} \mathcal{L}_{3}(\Phi, \Psi) & =f^{6} .
\end{aligned}
$$

Inicialmente, tomando o produto interno em $\mathcal{H}$ da equação resolvente (4.19) com $U$ obtemos

$$
i \beta\|U\|_{\mathcal{H}}^{2}-(\mathcal{A} U, U)_{\mathcal{H}}=(F, U)_{\mathcal{H}}
$$

Assim, tomando a parte real em (4.26) e usando (4.16) chegamos a seguinte estimativa

$$
\begin{aligned}
D_{0}\|\nabla W\|_{2}^{2} & +D_{1}(1-\mu)\left[\left\|\Psi_{x}\right\|_{2}^{2}+\left\|\Phi_{y}\right\|_{2}^{2}\right] \\
& +D_{1} \mu\left\|\Psi_{x}+\Phi_{y}\right\|_{2}^{2}+D_{1}\left(\frac{1-\mu}{2}\right)\left\|\Phi_{x}+\Psi_{y}\right\|_{2}^{2} \leq\|U\|_{\mathcal{H}}\|F\|_{\mathcal{H}} .
\end{aligned}
$$


Prova da condição (4.17). Provar que $i \mathbb{R} \subset \rho(\mathcal{A})$ é equivalente a provar que o operador $i \beta I_{d}-\mathcal{A}$ é invertível para qualquer $\beta \in \mathbb{R}$. Note que isto já é verdadeiro para $\beta=0$. A seguir, vamos mostrar que vale a seguinte implicação

$$
\left(i \beta I_{d}-\mathcal{A}\right) U=0 \Longrightarrow U=0,
$$

para $\beta$ real não nulo. Isto é suficiente para concluir o desejado. De fato, para $F=(0,0,0,0,0,0)^{t}$ em $\mathcal{H}$ segue de (4.27) que

$$
\begin{aligned}
D_{0}\|\nabla W\|_{2}^{2} & +D_{1}(1-\mu)\left[\left\|\Psi_{x}\right\|_{2}^{2}+\left\|\Phi_{y}\right\|_{2}^{2}\right] \\
& +D_{1} \mu\left\|\Psi_{x}+\Phi_{y}\right\|_{2}^{2}+D_{1}\left(\frac{1-\mu}{2}\right)\left\|\Phi_{x}+\Psi_{y}\right\|_{2}^{2}=0 .
\end{aligned}
$$

Então $\|\nabla W\|_{2}^{2}=0$ e pela desigualdade de Poincaré obtemos $W=0$. Retornando a (4.29) vem que

$$
(1-\mu)\left[\left\|\Psi_{x}\right\|_{2}^{2}+\left\|\Phi_{y}\right\|_{2}^{2}\right]+\mu\left\|\Psi_{x}+\Phi_{y}\right\|_{2}^{2}+\left(\frac{1-\mu}{2}\right)\left\|\Phi_{x}+\Psi_{y}\right\|_{2}^{2}=0 .
$$

Do Lema 4.2.1, existe uma constante $\alpha_{0}>0$ tal que

$$
\begin{aligned}
\alpha_{0}\left[\|\Psi\|_{H^{1}(\Omega)}^{2}+\|\Phi\|_{H^{1}(\Omega)}^{2}\right] \leq & D\left\{(1-\mu)\left[\left\|\Psi_{x}\right\|_{2}^{2}+\left\|\Phi_{y}\right\|_{2}^{2}\right]+\mu\left\|\Psi_{x}+\Phi_{y}\right\|_{2}^{2}\right. \\
& \left.+\left(\frac{1-\mu}{2}\right)\left\|\Phi_{x}+\Psi_{y}\right\|_{2}^{2}\right\}=0
\end{aligned}
$$

e com maior razão temos $\Psi=\Phi=0$. Substituindo $W=\Psi=\Phi=0$ em (4.20), (4.22) e (4.24), respectivamente (e relembrando que $F=0$ ), resulta que $w=\psi=\varphi=0$. Portanto, $U=0$ e a condição (4.28) está assegurada.

Prova da condição (4.18). Vamos mostrar que existe uma constante $C>0$ independente de $\beta$ tal que

$$
\left\|\beta\left(i \beta I_{d}-\mathcal{A}\right)^{-1}\right\| \leq C \quad \text { quando } \quad|\beta| \rightarrow \infty .
$$

Contudo, como $i \mathbb{R} \subset \rho(\mathcal{A})$, então usando a equação resolvente (4.19), para mostrar (4.30) é suficiente mostrar que

$$
|\beta||| U\left\|_{\mathcal{H}} \leq C|| F\right\|_{\mathcal{H}} \quad \text { quando } \quad|\beta| \rightarrow \infty .
$$

Assim sendo, vamos mostrar a seguir que a condição (4.31) é satisfeita. Com efeito, tomando a parte imaginária em (4.26) obtemos, depois de tomar o módulo, a seguinte desigualdade

$$
\left.|\beta||| U\left\|_{\mathcal{H}}^{2} \leq\left|\mathcal{I} m(\mathcal{A} U, U)_{\mathcal{H}}\right|+\right\| U\right|_{\mathcal{H}}|| F \|_{\mathcal{H}} .
$$

Utilizando a expressão para $(\mathcal{A} U, U)_{\mathcal{H}}$ obtida na seção 4.2 (logo acima), temos

$$
\begin{aligned}
\left|\mathcal{I} m(\mathcal{A} U, U)_{\mathcal{H}}\right| \leq & 2 K\left\|\psi+w_{x}\right\|_{2}\left\|\Psi+W_{x}\right\|_{2}+2 K\left\|\varphi+w_{y}\right\|_{2}\left\|\Phi+W_{y}\right\|_{2} \\
& +2 D\left\|\psi_{x}\right\|_{2}\left\|\Psi_{x}\right\|_{2}+2 D\left\|\varphi_{y}\right\|_{2}\left\|\Phi_{y}\right\|_{2}+2 D \mu\left\|\varphi_{y}\right\|_{2}\left\|\Psi_{x}\right\|_{2} \\
& +2 D \mu\left\|\psi_{x}\right\|_{2}\left\|\Phi_{y}\right\|_{2}+D(1-\mu)\left\|\varphi_{x}+\psi_{y}\right\|_{2}\left\|\Psi_{y}+\Phi_{x}\right\|_{2} .
\end{aligned}
$$


Agora, lembrando da norma para $U$ em $\mathcal{H}$ e usando a estimativa (4.27) repetidas vezes em (4.33), segue que

$$
\left|\mathcal{I} m(\mathcal{A} U, U)_{\mathcal{H}}\right| \leq C_{0}|| U\left\|_{\mathcal{H}}^{2}+C_{0}\right\| U\left\|_{\mathcal{H}}^{3 / 2}\right\| F \|_{\mathcal{H}}^{1 / 2},
$$

para alguma constante $C_{0}>0$. Substituindo (4.34) em (4.32) resulta que

$$
|\beta|\|U\|_{\mathcal{H}}^{2} \leq C_{0}\|U\|_{\mathcal{H}}^{2}+C_{0}\|U\|_{\mathcal{H}}^{3 / 2}\|F\|_{\mathcal{H}}^{1 / 2}+\|U\|_{\mathcal{H}}\|F\|_{\mathcal{H}},
$$

ou ainda,

$$
|\beta||| U\left\|_{\mathcal{H}} \leq C_{0}\right\| U\left\|_{\mathcal{H}}+C_{0}|| U\right\|_{\mathcal{H}}^{1 / 2}\|F\|_{\mathcal{H}}^{1 / 2}+\|F\|_{\mathcal{H}},
$$

onde a constante $C_{0}>0$ independe de $\beta$. Usando a desigualdade de Young, existe uma constante $C>0$ tal que

$$
(|\beta|-C)|| U\left\|_{\mathcal{H}} \leq C|| F\right\|_{\mathcal{H}}
$$

Por fim, tomando $|\beta|$ grande o suficiente em (4.36) (escolhendo por exemplo $|\beta| \geq 2 C$ ) segue o desejado, uma vez que (4.31) é requerida para valores grandes de $|\beta|$. Portanto, a prova do Teorema 4.3.1 está completa.

\subsubsection{Estabilidade exponencial}

Como consequência, $S(t)=e^{\mathcal{A} t}$ é exponencialmente estável. Mais precisamente, temos o

Corolário 4.3.2. Sob as condições do Teorema 4.2.2, se $D_{0}, D_{1}>0$, então o $C_{0}$-semigrupo de contrações $S(t)=e^{\mathcal{A} t}$ correspondente ao sistema de Mindlin-Timoshenko é exponencialmente estável.

Prova. É suficiente notar que a analiticidade de $S(t)$ fornecida pelo Teorema 4.3.1 implica nas condições necessárias do Teorema 1.5.5, a saber, as condições (1.10) e (1.11) da seção 1.5 do Capítulo 1. De fato, a condição (1.10) é a mesma que (1.8) que já foi verificada neste contexto. Para tratar da condição (1.11) basta mostrar que existe uma constante $C>0$ independente de $\beta$ tal que

$$
\left\|\left(i \beta I_{d}-\mathcal{A}\right)^{-1}\right\| \leq C \quad \text { quando } \quad|\beta| \rightarrow \infty .
$$

Como $i \mathbb{R} \subset \rho(\mathcal{A})$, então novamente da equação resolvente (4.19) basta mostrar que

$$
\|U\|_{\mathcal{H}} \leq C\|F\|_{\mathcal{H}} \quad \text { quando } \quad|\beta| \rightarrow \infty .
$$

Contudo, retornando à estimativa (4.31) obtida na prova do Teorema 4.3.1 inferimos que

$$
|\beta|\|U\|_{\mathcal{H}} \leq C|| F \|_{\mathcal{H}} \quad \text { quando } \quad|\beta| \rightarrow \infty,
$$

para alguma constante $C>0$. Como a desigualdade (4.38) é requerida para $|\beta|$ suficientemente grande, então podemos considerar que $|\beta| \geq 1$, sem perda de generalidade. Fazendo isto, segue diretamente que

$$
\|U\|_{\mathcal{H}} \leq \frac{C}{|\beta|}\|F\|_{\mathcal{H}} \leq C\|F\|_{\mathcal{H}},
$$

para $|\beta| \geq 1$ suficientemente grande, o que prova o Corolário 4.3.2. 


\subsection{Falta de estabilidade exponencial}

Nesta seção vamos mostrar que o sistema de Mindlin-Timoshenko perde estabilidade exponencial quando consideramos $D_{0}=0$ e $D_{1}>0$, ou seja, que o semigrupo correspondente ao sistema não é exponencialmente estável. Este fato nos permite dizer que o sistema também perde a analiticidade. Com isto, notamos que $D_{0}>0$ é uma condição necessária e suficiente para se obter estabilidade exponencial (e analiticidade) quando fixamos $D_{1}>0$.

Para comprovar o desejado, basta exibir um contraexemplo em que o sistema de MindlinTimshenko, com pelo menos uma das condições de contorno, não seja exponencialmente estável. Nesta ocasião, será considerado o sistema de Mindlin-Timoshenko com as condições mistas (4.9) na fronteira. Em resumo, temos o

Teorema 4.4.1. Sob as condições do Teorema 4.2.2, se $D_{0}=0$ e $D_{1}>0$, então o $C_{0}$-semigrupo de contrações $S(t)=e^{\mathcal{A} t}$ correspondente ao sistema de Mindlin-Timoshenko não é exponencialmente estável.

Prova. De acordo com o Teorema 1.5.5, basta mostrar que uma das condições (1.10) ou (1.11) não vale. No que segue vamos verificar que a condição (1.11) não se verifica. Mais precisamente, mostraremos que existe uma sequência de números reais $\left(\beta_{n}\right)_{n \in \mathbb{N}}$ com

$$
\lim _{n \rightarrow \infty}\left|\beta_{n}\right|=\infty
$$

e uma sequência $U_{n}=\left(w_{n}, W_{n}, \psi_{n}, \Psi_{n}, \varphi_{n}, \Phi_{n}\right)^{t}$ satisfazendo a equação resolvente

$$
\left(i \beta_{n} I_{d}-\mathcal{A}\right) U_{n}=F_{n}
$$

para funções limitadas $\left(F_{n}\right) \subset \mathcal{H}_{2}\left(\left\|F_{n}\right\|_{\mathcal{H}_{2}} \leq 1\right)$ tais que

$$
\lim _{n \rightarrow \infty}\left\|U_{n}\right\|_{\mathcal{H}_{2}}=\lim _{n \rightarrow \infty}\left\|\left(i \beta_{n} I_{d}-\mathcal{A}\right)^{-1} F_{n}\right\|_{\mathcal{H}_{2}}=\infty
$$

Sem perda de generalidade podemos considerar $\Omega \subset \mathbb{R}^{2}$ como sendo $\Omega=[0, \pi] \times[0, \pi]$ com fronteira $\Gamma=\bar{\Gamma}_{1} \cup \bar{\Gamma}_{2}$, onde aqui

$$
\Gamma_{1}=\{(x, y) ; 0<x<\pi, y=0, \pi\} \quad \text { e } \quad \Gamma_{2}=\{(x, y) ; 0<y<\pi, x=0, \pi\} .
$$

Para cada $n \in \mathbb{N}$, tomamos

$$
F_{n}=(0, \underbrace{\alpha_{1} \sin (n x) \sin (n y)}_{:=f_{n}^{2}}, 0, \underbrace{\alpha_{2} \cos (n x) \sin (n y)}_{:=f_{n}^{4}}, 0, \underbrace{\alpha_{3} \sin (n x) \cos (n y)}_{:=f_{n}^{6}})^{t},
$$


onde $\alpha_{1}, \alpha_{2}, \alpha_{3} \in \mathbb{R}$. Então a equação resolvente $i \beta U-\mathcal{A} U=F_{n}$, com $U=(w, W, \psi, \Psi, \varphi, \Phi)^{t}$, pode ser reescrita em termos de suas equações componentes como segue

$$
\begin{aligned}
i \beta w-W & =0 \\
i \beta W-\frac{K}{\rho_{1}}\left[\left(\psi+w_{x}\right)_{x}+\left(\varphi+w_{y}\right)_{y}\right] & =f_{n}^{2} \\
i \beta \psi-\Psi & =0 \\
i \beta \Psi-\frac{D}{\rho_{2}} \mathcal{L}_{2}(\psi, \varphi)+\frac{K}{\rho_{2}}\left(\psi+w_{x}\right)-\frac{D_{1}}{\rho_{2}} \mathcal{L}_{2}(\Psi, \Phi) & =f_{n}^{4} \\
i \beta \varphi-\Phi & =0 \\
i \beta \Phi-\frac{D}{\rho_{2}} \mathcal{L}_{3}(\varphi, \psi)+\frac{K}{\rho_{2}}\left(\varphi+w_{y}\right)-\frac{D_{1}}{\rho_{2}} \mathcal{L}_{3}(\Phi, \Psi) & =f_{n}^{6},
\end{aligned}
$$

onde relembramos que agora $D_{0}=0$. Sendo assim,

$$
W=i \beta w, \quad \Psi=i \beta \psi \quad \text { e } \quad \Phi=i \beta \varphi .
$$

Usando (4.42) segue facilmente que

$$
\mathcal{L}_{2}(\Psi, \Phi)=i \beta \mathcal{L}_{2}(\psi, \varphi), \quad \mathcal{L}_{3}(\Phi, \Psi)=i \beta \mathcal{L}_{3}(\varphi, \psi)
$$

e assim (4.41) é equivalente ao seguinte sistema em $w, \psi$ and $\varphi$.

$$
\begin{array}{r}
-\beta^{2} \rho_{1} w-K\left(\psi+w_{x}\right)_{x}-K\left(\varphi+w_{y}\right)_{y}=\rho_{1} f_{n}^{2} \\
-\beta^{2} \rho_{2} \psi-\left(D+i D_{1} \beta\right)\left(\psi_{x x}+\frac{1-\mu}{2} \psi_{y y}+\frac{1+\mu}{2} \varphi_{x y}\right)+K\left(\psi+w_{x}\right)=\rho_{2} f_{n}^{4} \\
-\beta^{2} \rho_{2} \varphi-\left(D+i D_{1} \beta\right)\left(\varphi_{y y}+\frac{1-\mu}{2} \varphi_{x x}+\frac{1+\mu}{2} \psi_{x y}\right)+K\left(\varphi+w_{y}\right)=\rho_{2} f_{n}^{6} .
\end{array}
$$

Impondo que (devido as condições de fronteira)

$$
\begin{aligned}
& w_{n}:=A \sin (n x) \sin (n y), \\
& \psi_{n}:=B \cos (n x) \sin (n y), \\
& \varphi_{n}:=C \sin (n x) \cos (n y),
\end{aligned}
$$

onde $A, B$ e $C$ serão determinadas posteriormente, então obter soluções para o sistema (4.43) é equivalente a encontrar soluções $A, B$ e $C$ para o seguinte sistema numérico

$$
\left\{\begin{array}{l}
p_{1}(\beta) A+K n B+K n C=\rho_{1} \alpha_{1} \\
K n A+p_{2}(\beta) B+p_{3}(\beta) C=\rho_{2} \alpha_{2} \\
K n A+p_{3}(\beta) B+p_{2}(\beta) C=\rho_{2} \alpha_{3},
\end{array}\right.
$$

onde

$$
\begin{aligned}
& p_{1}(\beta):=-\beta^{2} \rho_{1}+2 K n^{2}, \\
& p_{2}(\beta):=-\beta^{2} \rho_{2}+\frac{3-\mu}{2}\left(D+i D_{1} \beta\right) n^{2}+K, \\
& p_{3}(\beta):=\frac{1+\mu}{2}\left(D+i D_{1} \beta\right) n^{2} .
\end{aligned}
$$


Pois bem, o que faremos a seguir é derminar soluções para (4.45) com escolhas convenientes. Em primeiro lugar, escolhendo

$$
\beta:=\beta_{n}=\sqrt{\frac{2 K}{\rho_{1}}} n, \quad n \in \mathbb{N}
$$

então $p_{1}(\beta) \equiv 0$ e reescrevendo (4.45) sob a forma matricial, obtemos

$$
\left(\begin{array}{ccc}
0 & K n & K n \\
K n & p_{2}(\beta) & p_{3}(\beta) \\
K n & p_{3}(\beta) & p_{2}(\beta)
\end{array}\right)\left(\begin{array}{c}
A \\
B \\
C
\end{array}\right)=\left(\begin{array}{c}
\rho_{1} \alpha_{1} \\
\rho_{2} \alpha_{2} \\
\rho_{2} \alpha_{3}
\end{array}\right) .
$$

Usando a regra de Cramer, as expressões para $p_{1}, p_{2}, p_{3}$ dadas acima e a escolha de $\beta$ em (4.46), concluimos que

$$
\begin{gathered}
A:=A_{n}=-\frac{\alpha_{1} \rho_{1}}{2 K n^{2}}+\frac{\left(\alpha_{2}+\alpha_{3}\right) \rho_{2}}{2 K n}+\frac{\left(\rho_{2} K-\rho_{1} D\right) \alpha_{1}}{K^{2}}-i \alpha_{1} D_{1} \sqrt{\frac{2 \rho_{1}}{K^{3}}} n, \\
B:=B_{n}=\frac{\left(\alpha_{2}-\alpha_{3}\right) \rho_{2}}{2 K+\Theta n^{2}+i \Theta_{1} n^{3}}+\frac{\alpha_{1} \rho_{1}}{2 K n}
\end{gathered}
$$

$\mathrm{e}$

$$
C:=C_{n}=\frac{\left(\alpha_{3}-\alpha_{2}\right) \rho_{2}}{2 K+\Theta n^{2}+i \Theta_{1} n^{3}}+\frac{\alpha_{1} \rho_{1}}{2 K n},
$$

onde, para facilitar a notação, colocamos

$$
\Theta=2 D(1-\mu)-4 K \frac{\rho_{2}}{\rho_{1}} \quad \text { e } \quad \Theta_{1}=2 D_{1}(1-\mu) \sqrt{\frac{2 K}{\rho_{1}}} .
$$

Com isto, obtemos que a sequência $\beta_{n} A_{n}$ satisfaz

$$
\lim _{n \rightarrow \infty}\left|\beta_{n} A_{n}\right|^{2}=\infty
$$

Usando (4.42) e (4.44), obtemos

$$
W_{n}=i \beta_{n} w_{n}=i \beta_{n} A_{n} \sin (n x) \sin (n y),
$$

de onde segue que

$$
\left\|U_{n}\right\|_{\mathcal{H}_{2}}^{2} \geq \rho_{1}\left\|W_{n}\right\|_{2}^{2}=\frac{\rho_{1} \pi^{2}}{4}\left|\beta_{n} A_{n}\right|^{2} .
$$

Logo, combinando (4.51) e (4.52) concluimos que

$$
\lim _{n \rightarrow \infty}\left\|U_{n}\right\|_{\mathcal{H}_{2}}=\infty
$$

o que prova a condição (4.40) como desejado. Portanto, o Teorema 4.4.1 está provado. 
Observação 4.4.1. A escolha da condição de fronteira mista (4.9) foi necessária para haver compatibilidade com as soluções apresentadas em (4.44). Notamos ainda que encontrar um contraexemplo que produz a falta de decaimento exponencial para o sistema de Mindlin-Timoshenko com condição de Dirichlet (4.8) na fronteira parece ser mais complicado, permanecendo um caso a ser determinado.

Observação 4.4.2. Dentre as constantes $A_{n}, B_{n}$ e $C_{n}$ que encontramos em (4.48)-(4.50), a que gera a falta de decaimento exponencial é $A_{n}$. Contudo, notamos também que a mesma constante possui um comportamento da forma $\left|A_{n}\right|=o\left(\left|\beta_{n}\right|^{k}\right)$ quando $\beta_{n} \rightarrow \infty$, qualquer que seja $k \geq 2$. Além disso, calculando a norma em $\mathcal{H}_{2}$ das soluções particulares $U_{n}$ obtidas na demonstração do Teorema 4.4.1, obtemos que ||$U_{n} \|_{\mathcal{H}_{2}} \leq C\left|\beta_{n}\right|^{k}$, para alguma constante $C>0$, para qualquer $k \geq 2$ e para $\beta_{n}$ suficientemente grande. Isto nos permite escrever que $\left\|U_{n}\right\|_{\mathcal{H}_{2}}=O\left(|\beta|^{k}\right)$ quando $\beta_{n} \rightarrow \infty$, para qualquer $k \geq 2$. Desta forma, de acordo com o resultados de estabilidade apresentado na seção 1.5 do capítulo 1, criamos a expectativa de que as soluções do problema de Mindlin-Timoshenko parcialmente dissipativo possuem decaimento polinomial. Esta previsão será confirmada na seção seguinte.

\subsection{Decaimento polinomial}

Nesta seção continuaremos considerando que $D_{0}=0$ e $D_{1}>0$. Neste caso, vamos mostrar que o sistema de Mindlin-Timoshenko é polinomialmente estável. Mais precisamente, vamos mostrar que a solução $U(t)=e^{\mathcal{A} t} U_{0}$ do problema abstrato (4.10) dada pelo Teorema 4.2.2 possui decaimento do tipo polinomial ao longo do tempo (em ambos os casos com relação às condições de fronteira). Além disso, verificaremos que a taxa de decaimento pode ser melhorada conforme a regularidade dos dados iniciais. Por outro lado, veremos também que se fixarmos os dados iniciais no domínio do operador $\mathcal{A}$ então a taxa de decaimento polinomial não pode ser melhorada, nos fornecendo otimalidade com respeito a taxa de decaimento.

Para assegurar a veracidade das afirmações acima vamos usar um resultado recente devido a Borichev \& Tomilov [14], o qual está expresso no Teorema 1.5.6 da seção 1.5 do Capítulo 1. Este resultado nos fornece equivalências que serão cruciais para concluir o decaimento polinomial, bem como a otimalidade.

Nosso ponto de partida é considerar novamente a equação resolvente

$$
i \beta U-\mathcal{A} U=F \quad \text { em } \quad \mathcal{H}, \quad U \in \mathcal{D}(\mathcal{A})
$$

De modo similar à estimativa (4.27), obtida na seção 4.3, e lembrando que $D_{0}=0$, obtemos

$$
\begin{aligned}
D_{1}(1-\mu)\left[\left\|\Psi_{x}\right\|_{2}^{2}+\left\|\Phi_{y}\right\|_{2}^{2}\right] & +D_{1} \mu\left\|\Psi_{x}+\Phi_{y}\right\|_{2}^{2} \\
& +D_{1}\left(\frac{1-\mu}{2}\right)\left\|\Phi_{x}+\Psi_{y}\right\|_{2}^{2} \leq\|U\|_{\mathcal{H}}\|F\|_{\mathcal{H}} .
\end{aligned}
$$


Para $F=\left(f^{1}, f^{2}, f^{3}, f^{4}, f^{5}, f^{6}\right)^{t} \in \mathcal{H}$, a equação resolvente em termos de suas componentes fica sob a forma

$$
\begin{aligned}
i \beta w-W & =f^{1} \\
i \beta W-\frac{K}{\rho_{1}}\left[\left(\psi+w_{x}\right)_{x}+\left(\varphi+w_{y}\right)_{y}\right] & =f^{2} \\
i \beta \psi-\Psi & =f^{3} \\
i \beta \Psi-\frac{D}{\rho_{2}} \mathcal{L}_{2}(\psi, \varphi)+\frac{K}{\rho_{2}}\left(\psi+w_{x}\right)-\frac{D_{1}}{\rho_{2}} \mathcal{L}_{2}(\Psi, \Phi) & =f^{4} \\
i \beta \varphi-\Phi & =f^{5} \\
i \beta \Phi-\frac{D}{\rho_{2}} \mathcal{L}_{3}(\varphi, \psi)+\frac{K}{\rho_{2}}\left(\varphi+w_{y}\right)-\frac{D_{1}}{\rho_{2}} \mathcal{L}_{3}(\Phi, \Psi) & =f^{6} .
\end{aligned}
$$

Observação 4.5.1. Neste caso devemos verificar novamente que $i \mathbb{R} \subset \rho(\mathcal{A})$. Para comprovar isso, é suficiente mostrar que a condição $i \beta U-\mathcal{A} U=0$ implica em $U=0$. De fato, tomando $F=\mathbf{0}$ em $\mathcal{H}$ segue de (4.53) que $\Psi=\Phi=0$, como verificado anteriormente (ver seção 4.3). Retornando às equações (4.56) e (4.58) obtemos $\psi=\varphi=0$. Mais ainda, usando equações (4.57) e (4.59) e também pela desigualdade de Poincaré (pois $w \in H_{0}^{1}(\Omega)$ em ambos os casos) temos $w=0$. Finalmente, de (4.54) vem que $W=0$, implicando que $U=0$, como era desejado.

A seguir apresentaremos alguns lemas auxiliares que nos permitirão confirmar nosso objetivo, a saber, o de obter um resultado sobre estabilidade polinomial. No que segue, com intuito de facilitar a notação, usaremos o mesmo parâmetro $C$ para denotar diferentes constantes positivas que surgirão nas estimativas.

Lema 4.5.1. Sob as notações acima, existe uma constante $C>0$ independente de $\beta$ tal que

$$
\|\Psi\|_{H^{1}(\Omega)}^{2}+\|\Phi\|_{H^{1}(\Omega)}^{2} \leq C\|U\|_{\mathcal{H}}\|F\|_{\mathcal{H}}
$$

$e$

$$
|\beta|^{2}\|\nabla \psi\|_{2}^{2}+|\beta|^{2}|| \nabla \varphi\left\|_{2}^{2} \leq C|| U\right\|_{\mathcal{H}}\|F\|_{\mathcal{H}}+C\|F\|_{\mathcal{H}}^{2} .
$$

Prova. Aplicando o Lema 4.2.1, existe uma constante $\alpha_{0}>0$ tal que

$$
\begin{aligned}
\alpha_{0}\left[\|\Psi\|_{H^{1}(\Omega)}^{2}+\|\Phi\|_{H^{1}(\Omega)}^{2}\right] \leq & D\left\{(1-\mu)\left[\left\|\Psi_{x}\right\|_{2}^{2}+\left\|\Phi_{y}\right\|_{2}^{2}\right]+\mu\left\|\Psi_{x}+\Phi_{y}\right\|_{2}^{2}\right. \\
& \left.+\left(\frac{1-\mu}{2}\right)\left\|\Phi_{x}+\Psi_{y}\right\|_{2}^{2}\right\} .
\end{aligned}
$$

Usando a estimativa (4.53), então (4.60) segue imediatamente.

Agora, derivando as equações (4.56) e (4.58), obtemos

$$
i \beta \psi_{x}-\Psi_{x}=f_{x}^{3}, \quad i \beta \psi_{y}-\Psi_{y}=f_{y}^{3}
$$

$\mathrm{e}$

$$
i \beta \varphi_{x}-\Phi_{x}=f_{x}^{5}, \quad i \beta \varphi_{y}-\Phi_{y}=f_{y}^{5}
$$


respectivamente. Assim, é simples verificar que

$$
\begin{aligned}
|\beta|^{2}\left(\left\|\psi_{x}\right\|_{2}^{2}+\left\|\varphi_{y}\right\|_{2}^{2}\right) & \leq 2\left(\left\|\Psi_{x}\right\|_{2}^{2}+\left\|\Phi_{y}\right\|_{2}^{2}\right)+2\left(\left\|f_{x}^{3}\right\|_{2}^{2}+\left\|f_{y}^{5}\right\|_{2}^{2}\right) \\
|\beta|^{2}\left\|\psi_{x}+\varphi_{y}\right\|_{2}^{2} & \leq 2\left\|\Psi_{x}+\Phi_{y}\right\|_{2}^{2}+2\left\|f_{x}^{3}+f_{y}^{5}\right\|_{2}^{2} \\
|\beta|^{2}\left\|\varphi_{x}+\psi_{y}\right\|_{2}^{2} & \leq 2\left\|\Phi_{x}+\Psi_{y}\right\|_{2}^{2}+2\left\|f_{y}^{3}+f_{x}^{5}\right\|_{2}^{2} .
\end{aligned}
$$

Logo, somando as expressões em (4.62) depois de multiplicá-las pelas devidas constantes, usando a norma definida em $\mathcal{H}$ para $F$ e a estimativa inicial (4.53), concluimos que existe uma constante $C>0$ dependendo somente dos coeficientes do sistema de Mindlin-Timoshenko tal que

$$
\begin{aligned}
|\beta|^{2}\left\{D ( 1 - \mu ) \left[\left\|\psi_{x}\right\|_{2}^{2}+\right.\right. & \left.\left\|\varphi_{y}\right\|_{2}^{2}\right]+D \mu\left\|\psi_{x}+\varphi_{y}\right\|_{2}^{2} \\
& \left.+D\left(\frac{1-\mu}{2}\right)\left\|\varphi_{x}+\psi_{y}\right\|_{2}^{2}\right\} \leq C\left[\|U\|_{\mathcal{H}}\|F\|_{\mathcal{H}}+\|F\|_{\mathcal{H}}^{2}\right] .
\end{aligned}
$$

Novamente pelo Lema 4.2.1, a estimativa (4.61) segue diretamente para alguma constante $C>0$.

Isto conclui a prova do Lema 4.5.1.

Lema 4.5.2. Sob as notações acima, existe uma constante $C>0$ independente de $\beta$ tal que

$$
\|\Psi\|_{2}^{2} \leq \frac{C}{|\beta|}\|w\|_{2}\|U\|_{\mathcal{H}}^{1 / 2}\|F\|_{\mathcal{H}}^{1 / 2}+\frac{C}{|\beta|}\|U\|_{\mathcal{H}}\|F\|_{\mathcal{H}}+\frac{C}{|\beta|}\|F\|_{\mathcal{H}}^{2}
$$

$e$

$$
\|\Phi\|_{2}^{2} \leq \frac{C}{|\beta|}\|w\|_{2}\|U\|_{\mathcal{H}}^{1 / 2}\|F\|_{\mathcal{H}}^{1 / 2}+\frac{C}{|\beta|}\|U\|_{\mathcal{H}}\|F\|_{\mathcal{H}}+\frac{C}{|\beta|}\|F\|_{\mathcal{H}}^{2},
$$

$\operatorname{para}|\beta| \geq 1$.

Prova. Inicialmente, afirmamos que existe uma constante $C>0$ de modo que:

$$
\begin{aligned}
|\beta|\|\Psi\|_{H^{-1}(\Omega)} & \leq C\left\{\|\nabla \psi\|_{2}+\|\nabla \varphi\|_{2}+\|w\|_{2}+\|\nabla \Phi\|_{2}+\|\Psi\|_{H^{1}(\Omega)}+\|F\|_{\mathcal{H}}\right\}, \\
|\beta|\|\Phi\|_{H^{-1}(\Omega)} & \leq C\left\{\|\nabla \psi\|_{2}+\|\nabla \varphi\|_{2}+\|w\|_{2}+\|\nabla \Psi\|_{2}+\|\Phi\|_{H^{1}(\Omega)}+\|F\|_{\mathcal{H}}\right\},
\end{aligned}
$$

onde lembramos que $H^{-1}(\Omega)$ menciona o espaço dual de $H_{0}^{1}(\Omega)$.

De fato, seja $v \in H_{0}^{1}(\Omega)$. Multiplicando (4.57) por $\bar{v}$, integrando sobre $\Omega$, usando integração por partes (fórmula de Green) e a equação (4.56), obtemos

$$
\begin{aligned}
i \rho_{2} \beta(\Psi, v)= & -D\left(\psi_{x}+\mu \varphi_{y}, v_{x}\right)-D\left(\frac{1-\mu}{2}\right)\left(\varphi_{x}+\psi_{y}, v_{y}\right)+K\left(w, v_{x}\right)+i \frac{K}{\beta}\left(\Psi+f^{3}, v\right) \\
& -D_{1}\left(\Psi_{x}+\mu \Phi_{y}, v_{x}\right)-D_{1}\left(\frac{1-\mu}{2}\right)\left(\Phi_{x}+\Psi_{y}, v_{y}\right)+\rho_{2}\left(f^{4}, v\right) .
\end{aligned}
$$

Passando ao módulo e usando as desigualdades de Poincaré, de Cauchy-Schwarz e triangular, existe uma constante $C>0$ que depende somente dos coeficientes do sistema de Mindlin-Timoshenko tal que

$$
|\beta||(\Psi, v)| \leq C\left\{\|\nabla \psi\|_{2}+\|\nabla \varphi\|_{2}+\|w\|_{2}+\|\nabla \Phi\|_{2}+\|\Psi\|_{H^{1}(\Omega)}+\|F\|_{\mathcal{H}}\right\}\|\nabla v\|_{2},
$$


para $|\beta| \geq 1$. Logo, tomando a norma em $H^{-1}(\Omega)$ segue que

$$
|\beta|\|\Psi\|_{H^{-1}(\Omega)} \leq C\left\{\|\nabla \psi\|_{2}+\|\nabla \varphi\|_{2}+\|w\|_{2}+\|\nabla \Phi\|_{2}+\|\Psi\|_{H^{1}(\Omega)}+\|F\|_{\mathcal{H}}\right\},
$$

o que mostra a desigualdade (4.65). Analogamente se comprova a desigualdade (4.66) usando as equações (4.58)-(4.59) e seguindo os passos acima.

Agora note que pela desigualdade de interpolação (ver Lions \& Magenes [67, Capítulo 1])

$$
\|\Psi\|_{2}^{2} \leq C_{0}\|\Psi\|_{H^{-1}(\Omega)}\|\Psi\|_{H^{1}(\Omega)},
$$

para alguma constante $C_{0}>0$. Disto e usando (4.65), a desigualdade de Young e as estimativas (4.60)-(4.61) do Lema 4.5.1, obtemos

$$
\begin{aligned}
\|\Psi\|_{2}^{2} \leq & \frac{C}{|\beta|}\left\{\|\nabla \psi\|_{2}+\|\nabla \varphi\|_{2}+\|w\|_{2}+\|\nabla \Phi\|_{2}+\|\Psi\|_{H^{1}(\Omega)}+\|F\|_{\mathcal{H}}\right\}\|\Psi\|_{H^{1}(\Omega)} \\
\leq & \frac{C}{|\beta|}\|w\|_{2}\|\Psi\|_{H^{1}(\Omega)}+\frac{C}{|\beta|}\left\{\|\nabla \psi\|_{2}+\|\nabla \varphi\|_{2}\right\}\|\Psi\|_{H^{1}(\Omega)} \\
& +\frac{C}{|\beta|}\left\{\|\nabla \Phi\|_{2}+\|\Psi\|_{H^{1}(\Omega)}\right\}\|\Psi\|_{H^{1}(\Omega)}+\frac{C}{|\beta|}\|F\|_{\mathcal{H}}\|\Psi\|_{H^{1}(\Omega)} \\
\leq & \frac{C}{|\beta|}\|w\|_{2}\|\Psi\|_{H^{1}(\Omega)}+\frac{C}{|\beta|}\left\{\|\nabla \psi\|_{2}^{2}+\|\nabla \varphi\|_{2}^{2}\right\} \\
& +\frac{C}{|\beta|}\left\{\|\nabla \Phi\|_{2}^{2}+\|\Psi\|_{H^{1}(\Omega)}^{2}\right\}+\frac{C}{|\beta|}\|F\|_{\mathcal{H}}^{2}+\frac{C}{|\beta|}\|\Psi\|_{H^{1}(\Omega)}^{2} \\
\leq & \frac{C}{|\beta|}\|w\|_{2}\|U\|_{\mathcal{H}^{\prime}}^{1 / 2}\|F\|_{\mathcal{H}}^{1 / 2}+\frac{C}{|\beta|}\|U\|_{\mathcal{H}}\|F\|_{\mathcal{H}}+\frac{C}{|\beta|}\|F\|_{\mathcal{H}}^{2},
\end{aligned}
$$

para alguma constante $C>0$ e $|\beta| \geq 1$, ou seja,

$$
\|\Psi\|_{2}^{2} \leq \frac{C}{|\beta|}\|w\|_{2}\|U\|_{\mathcal{H}}^{1 / 2}\|F\|_{\mathcal{H}}^{1 / 2}+\frac{C}{|\beta|}\|U\|_{\mathcal{H}}\|F\|_{\mathcal{H}}+\frac{C}{|\beta|}\|F\|_{\mathcal{H}}^{2}
$$

Isto prova a estimativa (4.63) como era desejado. Da mesma forma se obtém a desigualdade (4.64) usando a desigualdade de interpolação para $\Phi$, a estimativa (4.66), a desigualdade de Young e o Lema 4.5.1, o que conclui a prova do Lema 4.5.2.

Lema 4.5.3. Sob as notações acima, existe uma constante $C>0$ independente de $\beta$ tal que

$$
\|w\|_{2} \leq C|\beta|\left\{\|\Psi\|_{H^{-1}(\Omega)}+\|\Phi\|_{H^{-1}(\Omega)}\right\}+C\|U\|_{\mathcal{H}}^{1 / 2}\|F\|_{\mathcal{H}}^{1 / 2}+C\|F\|_{\mathcal{H}},
$$

$\operatorname{para}|\beta| \geq 1$.

Prova. Derivando as equações componentes (4.57) e (4.59) com respeito a $x$ e $y$, respectivamente, reajustando os termos e somando as expressões resultantes obtemos que

$$
-\Delta w=\mathcal{F}
$$


onde

$$
\begin{aligned}
\mathcal{F}= & i \frac{\rho_{2}}{K} \beta \Psi_{x}-\frac{D}{K} \mathcal{L}_{2}(\psi, \varphi)_{x}+\psi_{x}-\frac{D_{1}}{K} \mathcal{L}_{2}(\Psi, \Phi)_{x}-\frac{\rho_{2}}{K} f_{x}^{4} \\
& +i \frac{\rho_{2}}{K} \beta \Phi_{y}-\frac{D}{K} \mathcal{L}_{3}(\varphi, \psi)_{y}+\varphi_{y}-\frac{D_{1}}{K} \mathcal{L}_{3}(\Phi, \Psi)_{y}-\frac{\rho_{2}}{K} f_{y}^{6}
\end{aligned}
$$

Note que a expressão para $\mathcal{F}$ faz sentido, uma vez que vale a equação (4.55). Assim, devido a regularidade dos problemas elípticos (ver Lions \& Magenes [67, Capítulo 2]) e das imersões de Sobolev, existe uma constante $C>0$ tal que

$$
\|w\|_{2} \leq C\|\mathcal{F}\|_{H^{-2}(\Omega)}
$$

onde lembramos que $H^{-2}(\Omega)$ denota o espaço dual de $H_{0}^{2}(\Omega)$. Resta estimar o lado direito de (4.68). Contudo, procedendo da mesma forma como na demonstração do Lema 4.5.2, considerando $v \in H_{0}^{2}(\Omega)$, utilizando integração por partes, a fórmula de Green, passando ao módulo, usando as desigualdades de Cauchy-Schwarz e triangular e também as inclusões $H_{0}^{2}(\Omega) \hookrightarrow H_{0}^{1}(\Omega) \hookrightarrow L^{2}(\Omega)$, obtemos:

$$
\begin{aligned}
|\langle\mathcal{F}, v\rangle| \leq & C|\beta|\left\{\|\Psi\|_{H^{-1}(\Omega)}+\|\Phi\|_{H^{-1}(\Omega)}\right\}\|v\|_{H_{0}^{2}(\Omega)} \\
& +C\left\{\|\nabla \Psi\|_{2}+\|\nabla \Phi\|_{2}+\|\nabla \psi\|_{2}+\|\nabla \varphi\|_{2}+\|F\|_{\mathcal{H}}\right\}\|v\|_{H_{0}^{2}(\Omega)}
\end{aligned}
$$

para alguma constante $C>0$ dependendo apenas dos coeficientes do sistema de MindlinTimoshenko. Disto segue que

$$
\begin{aligned}
\|\mathcal{F}\|_{H^{-2}(\Omega)} \leq & C|\beta|\left\{\|\Psi\|_{H^{-1}(\Omega)}+\|\Phi\|_{H^{-1}(\Omega)}\right\} \\
& +C\left\{\|\nabla \Psi\|_{2}+\|\nabla \Phi\|_{2}+\|\nabla \psi\|_{2}+\|\nabla \varphi\|_{2}+\|F\|_{\mathcal{H}}\right\} .
\end{aligned}
$$

Combinando (4.68) e (4.69) e usando o Lema 4.5.1, obtemos (4.67) e o Lema 4.5.3 segue.

Lema 4.5.4. Sob as notações acima, existe uma constante $C>0$ independente de $\beta$ tal que

$$
\|\nabla w\|_{2}^{2} \leq C|\beta|^{2}|| U\left\|_{\mathcal{H}}\right\| F\left\|_{\mathcal{H}}+C|\beta|^{3}|| F\right\|_{\mathcal{H}}^{2}
$$

$e$

$$
\|W\|_{2}^{2} \leq C|\beta|^{2}\|U\|_{\mathcal{H}}\|F\|_{\mathcal{H}}+C|\beta|^{3}|| F \|_{\mathcal{H}}^{2}
$$

$\operatorname{para}|\beta| \geq 1$.

Prova. Afirmamos inicialmente que existe uma constante $C>0$ tal que

$$
\begin{aligned}
|\beta|\|\Psi\|_{H^{-2}(\Omega)} & \leq C\left\{\|w\|_{H^{-1}(\Omega)}+\|\Psi\|_{2}+\|\Phi\|_{2}+\|F\|_{\mathcal{H}}\right\} \\
|\beta|\|\Phi\|_{H^{-2}(\Omega)} & \leq C\left\{\|w\|_{H^{-1}(\Omega)}+\|\Psi\|_{2}+\|\Phi\|_{2}+\|F\|_{\mathcal{H}}\right\}
\end{aligned}
$$

onde novamente $H^{-2}(\Omega)=\left[H_{0}^{2}(\Omega)\right]^{\prime}$. 
Com efeito, seja $v \in H_{0}^{2}(\Omega)$. Multiplicando (4.57) por $\bar{v}$, integrando sobre $\Omega$, usando integração por partes e a equação (4.56), obtemos

$$
\begin{aligned}
i \rho_{2} \beta(\Psi, v)= & -D\left(\psi_{x}+\mu \varphi_{y}, v_{x}\right)-D\left(\frac{1-\mu}{2}\right)\left(\varphi_{x}+\psi_{y}, v_{y}\right)+K\left(w, v_{x}\right)+i \frac{K}{\beta}\left(\Psi+f^{3}, v\right) \\
& -D_{1}\left(\Psi_{x}+\mu \Phi_{y}, v_{x}\right)-D_{1}\left(\frac{1-\mu}{2}\right)\left(\Phi_{x}+\Psi_{y}, v_{y}\right)+\rho_{2}\left(f^{4}, v\right) .
\end{aligned}
$$

Integrando novamente por partes,

$$
\begin{aligned}
i \rho_{2} \beta(\Psi, v)= & \left(D \psi+D_{1} \Psi, v_{x x}\right)+\mu\left(D \varphi+D_{1} \Phi, v_{x y}\right)+\left(\frac{1-\mu}{2}\right)\left(D \varphi+D_{1} \Phi, v_{y x}\right) \\
& +\left(\frac{1-\mu}{2}\right)\left(D \psi+D_{1} \Psi, v_{y y}\right)+K\left(w, v_{x}\right)+i \frac{K}{\beta}\left(\Psi+f^{3}, v\right)+\rho_{2}\left(f^{4}, v\right) .
\end{aligned}
$$

Passando ao módulo e usando as desigualdades de Cauchy-Schwarz e triangular, então existe uma constante $C>0$ que depende somente dos coeficientes do sistema de Mindlin-Timoshenko tal que

$$
|\beta||(\Psi, v)| \leq C\left\{\|w\|_{H^{-1}(\Omega)}+\|\psi\|_{2}+\|\varphi\|_{2}+\|\Phi\|_{2}+\|\Psi\|_{2}+\|F\|_{\mathcal{H}}\right\}\|v\|_{H_{0}^{2}(\Omega)},
$$

para $|\beta| \geq 1$, onde usamos também que $H_{0}^{2}(\Omega) \hookrightarrow H_{0}^{1}(\Omega) \hookrightarrow L^{2}(\Omega)$. Usando as equações componentes (4.56) e (4.58) vem que

$$
|\beta||(\Psi, v)| \leq C\left\{\|w\|_{H^{-1}(\Omega)}+\|\Phi\|_{2}+\|\Psi\|_{2}+\|F\|_{\mathcal{H}}\right\}\|v\|_{H_{0}^{2}(\Omega)},
$$

para $|\beta| \geq 1$ e $C>0$ independente de $\beta$. Logo, tomando a norma em $H^{-2}(\Omega)$ segue que

$$
|\beta|\|\Psi\|_{H^{-2}(\Omega)} \leq C\left\{\|w\|_{H^{-1}(\Omega)}+\|\Psi\|_{2}+\|\Phi\|_{2}+\|F\|_{\mathcal{H}}\right\} .
$$

Isto mostra a desigualdade (4.72). De modo análogo mostra-se (4.73).

Agora note que usando novamente a desigualdade de interpolação, a estimativa (4.72) e as equações componentes (4.54)-(4.55), obtemos que

$$
\begin{aligned}
\|\Psi\|_{H^{-1}(\Omega)} & \leq C\|\Psi\|_{H^{-2}(\Omega)}^{1 / 2}\|\Psi\|_{2}^{1 / 2} \\
& \leq \frac{C}{|\beta|^{1 / 2}}\left\{\|w\|_{H^{-1}(\Omega)}^{1 / 2}+\|\Psi\|_{2}^{1 / 2}+\|\Phi\|_{2}^{1 / 2}+\|F\|_{\mathcal{H}}^{1 / 2}\right\}\|\Psi\|_{2}^{1 / 2} \\
& \leq \frac{C}{|\beta|^{1 / 2}}\left\{\frac{1}{|\beta|^{1 / 2}}\|W\|_{H^{-1}(\Omega)}^{1 / 2}\|\Psi\|_{2}^{1 / 2}+\|\Psi\|_{2}+\|\Phi\|_{2}+\|F\|_{\mathcal{H}}^{1 / 2}\|\Psi\|_{2}^{1 / 2}\right\} \\
& \leq \frac{C}{|\beta|^{1 / 2}}\left\{\frac{1}{|\beta|}\|\nabla w\|_{2}^{1 / 2}\|\Psi\|_{2}^{1 / 2}+\|\Psi\|_{2}+\|\Phi\|_{2}+\|F\|_{\mathcal{H}}^{1 / 2}\|\Psi\|_{2}^{1 / 2}\right\} \\
& \leq \frac{C}{|\beta|^{3 / 2}}\|\nabla w\|_{2}^{1 / 2}\|\Psi\|_{2}^{1 / 2}+\frac{C}{|\beta|^{1 / 2}}\left\{\|\Psi\|_{2}+\|\Phi\|_{2}+\|F\|_{\mathcal{H}}^{1 / 2}\|\Psi\|_{2}^{1 / 2}\right\},
\end{aligned}
$$

para alguma constante $C>0$ que independe de $\beta$, ou seja,

$$
\|\Psi\|_{H^{-1}(\Omega)} \leq \frac{C}{|\beta|^{3 / 2}}\|\nabla w\|_{2}^{1 / 2}\|\Psi\|_{2}^{1 / 2}+\frac{C}{|\beta|^{1 / 2}}\left\{\|\Psi\|_{2}+\|\Phi\|_{2}+\|F\|_{\mathcal{H}}^{1 / 2}\|\Psi\|_{2}^{1 / 2}\right\} .
$$


De modo análogo obtemos

$$
\|\Phi\|_{H^{-1}(\Omega)} \leq \frac{C}{|\beta|^{3 / 2}}\|\nabla w\|_{2}^{1 / 2}\|\Phi\|_{2}^{1 / 2}+\frac{C}{|\beta|^{1 / 2}}\left\{\|\Psi\|_{2}+\|\Phi\|_{2}+\|F\|_{\mathcal{H}}^{1 / 2}\|\Psi\|_{2}^{1 / 2}\right\} .
$$

Combinando o Lema 4.5.3 com as estimativas (4.74)-(4.75) e usando a desigualdade de Young vem que

$$
\begin{aligned}
\|w\|_{2} \leq & \frac{C}{|\beta|^{1 / 2}}\|\nabla w\|_{2}^{1 / 2}\left\{\|\Psi\|_{2}^{1 / 2}+\|\Phi\|_{2}^{1 / 2}\right\}+C|\beta|^{1 / 2}\left\{\|\Psi\|_{2}+\|\Phi\|_{2}\right\} \\
& +C\|U\|_{\mathcal{H}}^{1 / 2}\|F\|_{\mathcal{H}}^{1 / 2}+C|\beta|^{1 / 2}\|F\|_{\mathcal{H}}
\end{aligned}
$$

onde $C>0$ é independente de $\beta$ e $|\beta| \geq 1$.

Aplicando o Lema 4.5.2 e a desigualdade de Young $\operatorname{com} \epsilon>0$ suficientemente pequeno, então

$$
\|w\|_{2} \leq \frac{C}{|\beta|^{1 / 2}}\|\nabla w\|_{2}^{1 / 2}\left\{\|\Psi\|_{2}^{1 / 2}+\|\Phi\|_{2}^{1 / 2}\right\}+C\|U\|_{\mathcal{H}}^{1 / 2}\|F\|_{\mathcal{H}}^{1 / 2}+C|\beta|^{1 / 2}\|F\|_{\mathcal{H}} .
$$

Usando novamente a desigualdade de Young com $\epsilon>0$ e o Lema 4.5.1, obtemos

$$
\|w\|_{2} \leq \frac{\epsilon}{|\beta|}\|\nabla w\|_{2}+C_{\epsilon}\|U\|_{\mathcal{H}}^{1 / 2}\|F\|_{\mathcal{H}}^{1 / 2}+C|\beta|^{1 / 2}\|F\|_{\mathcal{H}}
$$

onde $C>0$ e $C_{\epsilon}>0$ são independentes de $\beta$ e $|\beta| \geq 1$. Retornando à equação componente (4.54) e usando (4.76), obtemos também

$$
\|W\|_{2} \leq \epsilon\|\nabla w\|_{2}+C_{\epsilon}|\beta|\|U\|_{\mathcal{H}}^{1 / 2}\|F\|_{\mathcal{H}}^{1 / 2}+C|\beta|^{3 / 2}\|F\|_{\mathcal{H}}
$$

Por outro lado, multiplicando (4.55) por $\bar{w}$ e integrando por partes sobre $\Omega$, temos

$$
\|\nabla w\|_{2}^{2}=-\frac{i \rho_{1}}{K} \beta(W, w)+\left(\psi_{x}, w\right)+\left(\varphi_{y}, w\right)+\frac{\rho_{1}}{K}\left(f^{2}, w\right) .
$$

Sendo assim, passando ao módulo e usando as desigualdades triangular, de Cauchy-Schwarz, de Poincaré, de Young e a equação componente (4.54), temos

$$
\begin{aligned}
\|\nabla w\|_{2}^{2} & \leq \frac{\rho_{1}}{K}|\beta|\|w\|_{2}\|W\|_{2}+\left\|\psi_{x}\right\|_{2}\|w\|_{2}+\left\|\varphi_{y}\right\|_{2}\|w\|_{2}+\frac{\rho_{1}}{K}\left\|f^{2}\right\|_{2}\|w\|_{2} \\
& \leq C\left\{\|W\|_{2}^{2}+C\|F\|_{\mathcal{H}}^{2}\right\}+C\left\{\|\nabla \psi\|_{2}+\|\nabla \varphi\|_{2}+\|F\|_{\mathcal{H}}\right\}\|\nabla w\|_{2},
\end{aligned}
$$

para alguma constante $C>0$ dependendo somente dos coeficientes do sistema de MindlinTimoshenko. Agora, usando a desigualdade de Young com $\epsilon>0$ suficientemente pequeno e aplicando o Lema 4.5.1, obtemos

$$
\|\nabla w\|_{2}^{2} \leq C\|W\|_{2}^{2}+C\|U\|_{\mathcal{H}}\|F\|_{\mathcal{H}}+C\|F\|_{\mathcal{H}}^{2},
$$

ou ainda,

$$
\|\nabla w\|_{2} \leq C\|W\|_{2}+C\|U\|_{\mathcal{H}}^{1 / 2}\|F\|_{\mathcal{H}}^{1 / 2}+C\|F\|_{\mathcal{H}}
$$


onde $C>0$ independe de $\beta$. Substituindo (4.79) em (4.77) e tomando $\epsilon>0$ suficiente pequeno, então existe uma constante $C>0$ independente de $\beta$ tal que

$$
\|W\|_{2}^{2} \leq C|\beta|^{2}|| U\left\|_{\mathcal{H}}\right\| F\left\|_{\mathcal{H}}+C|\beta|^{3}\right\| F \|_{\mathcal{H}}^{2},
$$

para $|\beta| \geq 1$. Isto prova a desigualdade (4.71) como desejado. Finalmente, substituindo esta última estimativa em (4.78) concluimos que

$$
\|\nabla w\|_{2}^{2} \leq C|\beta|^{2}\|U\|_{\mathcal{H}}\|F\|_{\mathcal{H}}+C|\beta|^{3}|| F \|_{\mathcal{H}}^{2},
$$

onde $|\beta| \geq 1$ e $C>0$ é independente de $\beta$.

Isto conclui a prova do Lema 4.5.4.

Lema 4.5.5. Sob as notações acima, existe uma constante $C>0$ independente de $\beta$ tal que

$$
\|U\|_{\mathcal{H}}^{2} \leq C|\beta|^{4}|| F \|_{\mathcal{H}}^{2},
$$

$\operatorname{para}|\beta| \geq 1$.

Prova. Em primeiro Lugar note que existe uma constante $C=C\left(\rho_{1}, \rho_{2}, K, D, \mu\right)>0$ dependendo apenas dos coeficientes do sistema de Mindlin-Timoshenko tal que

$$
\|U\|_{\mathcal{H}}^{2} \leq C\left\{\|W\|_{2}^{2}+\|\Psi\|_{2}^{2}+\|\Phi\|_{2}^{2}+\|\psi\|_{2}^{2}+\|\varphi\|_{2}^{2}+\|\nabla w\|_{2}^{2}+\|\nabla \psi\|_{2}^{2}+\|\nabla \varphi\|_{2}^{2}\right\} .
$$

Logo, usando as equações componentes (4.56) e (4.58) e aplicando os Lemas 4.5.1 e 4.5.4, obtemos

$$
\|U\|_{\mathcal{H}}^{2} \leq C|\beta|^{2}\|U\|_{\mathcal{H}}\|F\|_{\mathcal{H}}+C|\beta|^{3}\|F\|_{\mathcal{H}}^{2},
$$

para alguma constante $C>0$ independente de $\beta$ e $|\beta| \geq 1$. Assim, usando a desigualdade de Young $\operatorname{com} \epsilon>0$ pequeno o suficiente, concluimos que

$$
\|U\|_{\mathcal{H}}^{2} \leq C|\beta|^{4}|| F \|_{\mathcal{H}}^{2},
$$

para $|\beta| \geq 1$ e $C>0$ dependendendo somente das constantes do sistema de Mindlin-Timoshenko e independente de $\beta$. Isto mostra a estimativa (4.81) e o Lema 4.5.5 está provado.

Em virtude dos lemas expostos acima, estamos em posição de provar o principal resultado desta seção que fornece o decaimento polinomial para a solução $U(t)=e^{\mathcal{A} t} U_{0}, t \geq 0$, relativa ao sistema de Mindlin-Timoshenko.

Teorema 4.5.6. Considere $D_{0}=0$ e $D_{1}>0$. Se $U_{0} \in \mathcal{D}(\mathcal{A})$, então existe uma constante $C>0$ tal que

$$
\left\|e^{\mathcal{A t}} U_{0}\right\|_{\mathcal{H}} \leq \frac{C}{t^{1 / 2}}\left\|U_{0}\right\|_{\mathcal{D}(\mathcal{A})} \quad \text { quando } \quad t \rightarrow \infty .
$$

Além disso, se $U_{0} \in \mathcal{D}\left(\mathcal{A}^{k}\right), k \in \mathbb{N}$, então existe uma constante $C_{k}>0$ tal que

$$
\left\|e^{\mathcal{A} t} U_{0}\right\|_{\mathcal{H}} \leq \frac{C_{k}}{t^{k / 2}}\left\|U_{0}\right\|_{\mathcal{D}\left(\mathcal{A}^{k}\right)} \quad \text { quando } \quad t \rightarrow \infty .
$$


Prova. Pela estimativa (4.81) do Lema 4.5.5 existe uma constante $C>0$ tal que

$$
\|U\|_{\mathcal{H}} \leq C|\beta|^{2}\|F\|_{\mathcal{H}},
$$

para $|\beta|$ grande o suficiente $(|\beta| \geq 1)$. Além disso, pela Observação 4.5.1 temos que $i \mathbb{R} \subset \rho(\mathcal{A})$. Então, usando a equação resolvente $\left(i \beta I_{d}-\mathcal{A}\right) U=F$, segue que

$$
\left\|\left(i \beta I_{d}-\mathcal{A}\right)^{-1}\right\| \leq C|\beta|^{2},
$$

para $|\beta|$ suficientemente grande. Isto implica que a condição $(B 1)$ do Teorema 1.5.6 vale com $\alpha=2$. Logo, pela condição $(B 4)$ do mesmo resultado vem que

$$
\left\|e^{\mathcal{A} t} \mathcal{A}^{-1}\right\|=O\left(t^{-1 / 2}\right), \quad t \rightarrow \infty,
$$

ou seja, existe uma constante $C>0$ tal que

$$
\left\|e^{\mathcal{A} t} \mathcal{A}^{-1}\right\| \leq \frac{C}{t^{1 / 2}} \quad \text { quando } \quad t \rightarrow \infty .
$$

Agora notamos que a equação $-\mathcal{A} U=F$ possui uma única solução $U_{0} \in \mathcal{D}(\mathcal{A})$. Assim,

$$
\|F\|_{\mathcal{H}}=\left\|\mathcal{A} U_{0}\right\|_{\mathcal{H}}=\left\|U_{0}\right\|_{\mathcal{D}(\mathcal{A}) .}
$$

Escrevendo $-U_{0}=\mathcal{A}^{-1} F$, então $-e^{\mathcal{A} t} U_{0}=e^{\mathcal{A} t} \mathcal{A}^{-1} F$. Portanto, combinando (4.84) e (4.85) concluimos que

$$
\left\|e^{\mathcal{A} t} U_{0}\right\|_{\mathcal{H}}=\left\|e^{\mathcal{A} t} \mathcal{A}^{-1} F\right\|_{\mathcal{H}} \leq\left\|e^{\mathcal{A} t} \mathcal{A}^{-1}\right\|\|F\|_{\mathcal{H}} \leq \frac{C}{t^{1 / 2}}\left\|U_{0}\right\|_{\mathcal{D}(\mathcal{A})} \quad \text { quando } \quad t \rightarrow \infty,
$$

ou seja, existe uma constante $C>0$ tal que

$$
\left\|e^{\mathcal{A} t} U_{0}\right\|_{\mathcal{H}} \leq \frac{C}{t^{1 / 2}}\left\|U_{0}\right\|_{\mathcal{D}(\mathcal{A})} \quad \text { quando } \quad t \rightarrow \infty
$$

o que prova (4.82) como desejado.

Além disso, usando indução sobre $k \in \mathbb{N}$ segue que $0 \in \rho\left(\mathcal{A}^{k}\right)$. Neste caso, a equação $-\mathcal{A}^{k} U=F$ possui uma única solução $U_{0} \in \mathcal{D}\left(\mathcal{A}^{k}\right)$. Isto implica que

$$
\|F\|_{\mathcal{H}}=\left\|\mathcal{A}^{k} U_{0}\right\|_{\mathcal{H}}=\left\|U_{0}\right\|_{\mathcal{D}\left(\mathcal{A}^{k}\right)} .
$$

Agora, escrevendo $-U_{0}=\mathcal{A}^{-k} F$, então

$$
-e^{\mathcal{A} t} U_{0}=e^{\mathcal{A} t} \mathcal{A}^{-k} F=\left(e^{\mathcal{A} t / k} \mathcal{A}^{-1}\right)^{k} F .
$$

Assim, combinando (4.84) e (4.86) concluimos que

$$
\left\|e^{\mathcal{A} t} U_{0}\right\|_{\mathcal{H}}=\left\|\left(e^{\mathcal{A} t / k} \mathcal{A}^{-1}\right)^{k} F\right\|_{\mathcal{H}} \leq\left\|e^{\mathcal{A} t / k} \mathcal{A}^{-1}\right\|^{k}\|F\|_{\mathcal{H}} \leq\left(\frac{C k^{1 / 2}}{t^{1 / 2}}\right)^{k}\left\|U_{0}\right\|_{\mathcal{D}\left(\mathcal{A}^{k}\right)}
$$

quando $t \rightarrow \infty$, ou seja, para cada $k \in \mathbb{N}$, existe uma constante $C_{k}>0$ tal que

$$
\left\|e^{\mathcal{A} t} U_{0}\right\|_{\mathcal{H}} \leq \frac{C_{k}}{t^{k / 2}}\left\|U_{0}\right\|_{\mathcal{D}\left(\mathcal{A}^{k}\right)} \quad \text { quando } \quad t \rightarrow \infty .
$$

Isto mostra a estimativa (4.83), o que encerra a prova do Teorema 4.5.6. 


\subsubsection{Otimalidade}

Notamos inicialmente que o Teorema 4.5.6 nos fornece uma taxa de decaimento polinomial melhor, conforme melhoramos a regularidade dos dados iniciais. Contudo, se fixarmos os dados iniciais em $\mathcal{D}(\mathcal{A})$, então veremos a seguir que a taxa $t^{-1 / 2}$ não pode ser melhorada. Mais precisamente, temos 0

Teorema 4.5.7. Sob as hipóteses do Teorema 4.5.6, se fixarmos $U_{0} \in \mathcal{D}(\mathcal{A})$ então a taxa de decaimento $t^{-1 / 2}$ é ótima.

Prova. No que segue, provaremos por contradição que a taxa $t^{-1 / 2}$ não pode ser melhorada. De fato, suponhamos que a taxa de decaimento polinomial pode ser melhorada para uma taxa da forma $t^{-1 /(2-\delta)} \operatorname{com} 0<\delta<2$, ou seja, que existe uma constante $C>0$ tal que

$$
\left\|e^{\mathcal{A} t} U_{0}\right\|_{\mathcal{H}} \leq \frac{C}{t^{1 /(2-\delta)}}\left\|U_{0}\right\|_{\mathcal{D}(\mathcal{A})} \quad \text { quando } \quad t \rightarrow \infty .
$$

Assim, para $F \in \mathcal{H}$, obtemos da equação resolvente que

$$
\left\|e^{\mathcal{A} t} \mathcal{A}^{-1} F\right\|_{\mathcal{H}}=\left\|e^{\mathcal{A} t} U_{0}\right\|_{\mathcal{H}} \leq \frac{C}{t^{1 /(2-\delta)}}\|F\|_{\mathcal{H}} \quad \text { quando } \quad t \rightarrow \infty
$$

de onde segue que

$$
\left\|e^{\mathcal{A} t} \mathcal{A}^{-1}\right\| \leq \frac{C}{t^{1 /(2-\delta)}} \quad \text { quando } \quad t \rightarrow \infty .
$$

Apicando o Teorema 1.5.6 $((B 4) \Rightarrow(B 1))$ vem que

$$
|\beta|^{\delta-2}||\left(i \beta I_{d}-\mathcal{A}\right)^{-1} \| \leq C \quad \text { quando } \quad \beta \rightarrow \infty .
$$

Logo, para $F \in \mathcal{H}$, usando novamente a equação resolvente (notando que $i \mathbb{R} \subset \rho(\mathcal{A})$ ) resulta que

$$
|\beta|^{\delta-2}|| U\left\|_{\mathcal{H}} \leq C|| F\right\|_{\mathcal{H}},
$$

para alguma constante $C>0$ e $|\beta|$ suficientemente grande.

Por outro lado, procedendo exatamente como na prova da falta de estabilidade exponencial (ver seção 4.4), assumimos sem perca de generalidade que $\Omega=[0, \pi] \times[0, \pi]$ e tomando

$$
F_{n}=\left(0, \alpha_{1} \sin (n x) \sin (n y), 0, \alpha_{2} \cos (n x) \sin (n y), 0, \alpha_{3} \sin (n x) \cos (n y)\right)^{t},
$$

com $n \in \mathbb{N}$ e $\alpha_{1}, \alpha_{2}, \alpha_{3} \in \mathbb{R}$, então a equação resolvente $\left(i \beta I_{d}-\mathcal{A}\right) U=F_{n}$ possui solução $U_{n}=\left(w_{n}, W_{n}, \psi_{n}, \Psi_{n}, \varphi_{n}, \Phi_{n}\right)^{t}$, onde as funções componentes são dadas em (4.42) e (4.44), com coeficientes $A_{n}, B_{n}$ e $C_{n}$ determinados em (4.48), (4.49) e (4.50), respectivamente.

Com isto, devido a parte imaginária de $A_{n}$ dada em (4.48), obtemos que a sequência $\beta_{n}^{\delta-1} A_{n}$ satisfaz

$$
\lim _{n \rightarrow \infty}\left|\beta_{n}^{\delta-1} A_{n}\right|=\infty
$$

Lembrando que neste caso

$$
W_{n}=i \beta_{n} w_{n}=i \beta_{n} A_{n} \sin (n x) \sin (n y),
$$


então, assim como em (4.52), obtemos

$$
\left\|U_{n}\right\|_{\mathcal{H}_{2}}^{2} \geq \rho_{1}\left\|W_{n}\right\|_{2}^{2}=\frac{\rho_{1} \pi^{2}}{4}\left|\beta_{n} A_{n}\right|^{2},
$$

de onde segue que

$$
\left|\beta_{n}\right|^{\delta-2}\left\|U_{n}\right\|_{\mathcal{H}_{2}} \geq \frac{\rho_{1}^{1 / 2} \pi}{2}\left|\beta_{n}^{\delta-1} A_{n}\right| .
$$

Deste modo, segue de (4.88) e (4.89) que

$$
\lim _{n \rightarrow \infty}\left|\beta_{n}\right|^{\delta-2}\left\|U_{n}\right\|_{\mathcal{H}_{2}}=\infty
$$

o que é um absurdo, pois contradiz (4.87). Portanto, a taxa $t^{-1 / 2}$ não pode ser melhorada. Isto nos dá a otimalidade desejada, concluindo a prova do Teorema 4.5.7. 


\section{Referências Bibliográficas}

[1] R. A. Adams \& J. J. F. Fournier, Sobolev Spaces, Second edition. Pure and Applied Mathematics (Amsterdam), 140. Elsevier/Academic Press, Amsterdam, 2003.

[2] L. An \& A. Peirce, The effect of microstructure on elastic-plastic models, SIAM J. Appl. Math. 54 (1994), no. 3, 708-730.

[3] L. An \& A. Peirce, A weakly nonlinear analysis of elastoplastic-microstructure models, SIAM J. Appl. Math. 55 (1995), no. 1, 136-155.

[4] D. Andrade, L. H. Fatori \& J. E. Muñoz Rivera, Nonlinear transmission problem with a dissipative boundary condition of memory type, Electron. J. Differential Equations 2006, No. $53,16 \mathrm{pp}$.

[5] D. Andrade, M. A. Jorge Silva \& T. F. Ma, Exponential stability for a plate equation with p-Laplacian and memory terms, Math. Meth. Appl. Sci. (aceito para publicação)

[6] D. Andrade \& J. E. Muñoz Rivera, Exponential decay of non-linear wave equation with a viscoelastic boundary condition, Math. Methods Appl. Sci. 23 (2000) 41-61.

[7] G. Andrews, On the existence of solutions to the equation $u_{t t}=u_{x x t}+\sigma\left(u_{x}\right)_{x}$, J. Differential Equations 35 (1980) 200-231.

[8] A. V. Babin \& M. I. Vishiki, Attractors of Evolution Equations, Studies in Mathematics and its Applications 25, North-Holland, Amsterdam, 1992.

[9] R. K. Barreto, E. C. Lapa \& J. E. Muñoz Rivera, Decay rates for viscoelastic plates with memory, J. Elasticity 44 (1996), no. 1, 61-87.

[10] R. K. Barreto \& J. E. Muñoz Rivera, Decay rates of solutions to thermoviscoelastic plates with memory, IMA J. Appl. Math. 60 (1998), no. 3, 263-283. 
[11] A. Bátkai, K.-J. Engel, J. Prüss and R. Schnaubelt, Polynomial stability of operator semigroups, Math. Nachr. 279 (2006), no. 13-14, 1425-1440.

[12] A. Benaissa \& A. Guesmia, Energy decay for the wave equations of $\phi$-Laplacian type with weakly nonlinear dissipation, Electron. J. Differential Equations (2008), no. 109, 1-22.

[13] A. C. Biazutti, On a nonlinear evolution equation and its applications, Nonlinear Anal. 24 (1995) 1221-1234.

[14] A. Borichev \& Y. Tomilov, Optimal polynomial decay of functions and operator semigroups, Math. Ann. 347 no. 2, 455-478 (2010).

[15] H. Brézis, Analyse fonctionnelle: Théorie et Applications, Paris, Masson, 1983.

[16] A. N. Carvalho, Sistemas dinâmicos não-lineares, Departamento de Matemática ICMC-USP, São Carlos, 2011.

[17] M. M. Cavalcanti \& V. N. Domingos Cavalcanti, Introdução à teoria das distribuições e aos espaços de Sobolev, EDUEM, Maringá, 2009.

[18] M. M. Cavalcanti, V. N. Domingos Cavalcanti \& J. Ferreira, Existence and uniform decay for a non-linear viscoelastic equation with strong damping, Math. Methods Appl. Sci. 24 (2001), no. $14,1043-1053$.

[19] M. M. Cavalcanti, V. N. Domingos Cavalcanti \& T. F. Ma, Exponential decay of the viscoelastic Euler-Bernoulli equation with a nonlocal dissipation in general domains, Differential Integral Equations 17 (2004) 495-510.

[20] M. M. Cavalcanti, H. Portillo Oquendo, Frictional versus viscoelastic damping in a semilinear wave equation, SIAM J. Control Optim. 42 (2003) 1310-1324.

[21] S. S. Ceron \& O. Lopes, $\alpha$-contractions and attractors for dissipative semilinear hyperbolic equations and systems, Ann. Math. Pura Appl. (4) 160 (1991), 193-206.

[22] I. Chueshov \& I. Lasiecka, Attractors for second order evolution equations with a nonlinear damping, J. Dyn. Diff. Eq., 16 (2004), 469-512.

[23] I. Chueshov \& I. Lasiecka, Existence, uniqueness of weak solutions and global attractors for a class of nonlinear 2D Kirchhoff-Boussinesq models, Discrete Contin. Dyn. Syst. 15 (2006), no. $3,777-809$.

[24] I. Chueshov \& I. Lasiecka, Long time dynamics of von Karman evolutions with thermal effects, Bol. Soc. Parana. Mat. (3) 25 (2007), no. 1-2, 37-54.

[25] I. Chueshov \& I. Lasiecka, Long-Time Behavior of Second Order Evolution Equations with Nonlinear Damping, Mem. Amer. Math. Soc. 195, no. 912, Providence, 2008. 
[26] I. Chueshov \& I. Lasiecka, Attractors and long time behavior of von Karman thermoelastic plates, Appl. Math. Optim. 58 (2008), no. 2, 195-241.

[27] I. Chueshov \& I. Lasiecka, On global attractor for 2D Kirchhoff-Boussinesq model with supercritical nonlinearity, Comm. Partial Differential Equations 36 (2011) 67-99.

[28] J. Clements, Existence theorems for a quasilinear evolution equation, SIAM J. Appl. Math. 26 (1974) 745-752.

[29] E. A. Coddington \& N. Levinson, Theory of Ordinary Differential Equations, McGraw-Hill Inc., New York, 1955.

[30] C. M. Dafermos, Asymptotic stability in viscoelasticity, Arch. Rational Mech. Anal. 37 (1970) 297-308.

[31] C. M. Dafermos \& J. A. Nohel, Energy methods for nonlinear hyperbolic Volterra integrodifferential equations, Comm. Partial Differential Equations 4 (1979) 219-278.

[32] Dang Dinh Ang \& A. Pham Ngoc Dinh, Strong solutions of a quasilinear wave equation with nonlinear damping, SIAM J. Math. Anal. 19 (1988) 337-347.

[33] R. Dautray \& J.-L. Lions, Mathematical Analysis and Numerical Methods for Science and Technology, Vol.5 - Evolution Problems I, Springer-Verlag, Berlin, 1992.

[34] M. Dreher, The wave equation for the p-Laplacian, Hokkaido Math. J. 36 (2007) 21-52.

[35] A. Eden, C. Foias, B. Nicolaenko \& R. Temam, Exponential attractors for dissipative evolution equations, Research in Applied Mathematics, vol. 37, John Wiley \& Sons, New York, 1994.

[36] L. C. Evans, Partial differential equations, Graduate Studies in Mathematics, vol. 19. American Mathematical Society, Providence, RI, 1998.

[37] K. Falconer, Fractal Geometry: Mathematical Foundations and Applications, Wiley, Chichester, 1990.

[38] L. H. Fatori \& T. F. Ma, A thermoelastic system of memory type in noncylindrical domains, Appl. Math. Comput. 200 (2008), no. 2, 583-589.

[39] L. H. Fatori \& R. N. Monteiro, The optimal decay rate for a weak dissipative Bresse system, Applied Mathematics Letters 25 (2012), 600-604.

[40] L. H. Fatori \& J. E. Muñoz Rivera, Rates of decay to weak thermoelastic Bresse system, IMA J. Appl. Math. 75 no. 6 (2010), 881-904.

[41] H. D. Fernández Sare, On the stability of Mindlin-Timoshenko plates, Quart. Appl. Math. 67 no. 2, 249-263 (2009). 
[42] C. Foias \& E. Olson, Finite fractal dimension and Hölder-Lipschitz parametrization, Indiana Univ. Math. J. 45 (1996), 603-616.

[43] G. B. Folland, Real analysis - Modern techniques and their applications, Second edition, Pure and Applied Mathematics, John Wiley \& Sons, Inc., New York, 1999.

[44] H. Gao \& T. F. Ma, Global solutions for a nonlinear wave equations with the p-Laplacian Operator, Electron. J. Qual. Theory Differ. Equ. 1999, No. 11, 13 pp.

[45] L. Gearhart, Spectral theory for contraction semigroups on Hilbert space, Trans. Amer. Math. Soc. 236 (1978), 385-394.

[46] J. M. Ghidaglia \& R. Temam, Attractors for damped nonlinear hyperbolic equations, J. Math. Pures Appl. 66, (1987) 273-319.

[47] C. Giorgi, M. Grasseli \& V. Pata, Well-posedness and longtime behavior of the phase-field model with memory in a history space setting, Quart. Appl. Math. 59 (2001) 701-736.

[48] C. Giorgi, A. Marzocchi \& V. Pata, Asymptotic behavior of a semilinear problem in heat conduction with memory, NoDEA Nonlinear Diff. Eq. Appl. 5, (1998) 333-354.

[49] C. Giorgi, A. Marzocchi \& V. Pata, Uniform attractors for a non-autonomous semilinear heat equation with memory, Quart. Appl. Math. 58 (2000) 661-683.

[50] C. Giorgi, J. E. Muñoz Rivera \& V. Pata, Global attractors for a semilinear hyperbolic equation in viscoelasticity, J. Math. Anal. Appl. 260 (2001) 83-99.

[51] C. Giorgi, V. Pata \& E. Vuk, On the extensible viscoelastic beam, Nonlinearity 21, (2008) 713-733.

[52] M. Grasselli \& V. Pata, Uniform attractors of nonautonomous dynamical systems with memory, Progr. Nonlinear Differential Equations Appl. 50 (2002) 155-178.

[53] J. M. Greenberg, R. C. MacCamy \& V. J. Mizel, On the existence, uniqueness, and stability of solutions of the equation $\sigma^{\prime}\left(u_{x}\right) u_{x x}+\lambda u_{x t x}=\rho_{0} u_{t t}$, J. Math. Mech. 17 (1968) 707-728.

[54] J. M. Greenberg, On the existence, uniqueness, and stability of solutions of the equation $\rho_{0} X_{t t}=E\left(X_{x}\right) X_{x x}+\lambda X_{x x t}$, J. Math. Anal. Appl. 25 (1969) 575-591.

[55] J. K. Hale, Asymptotic behavior of dissipative systems, Mathematical Surveys and Monographs, 25. American Mathematical Society, Providence, RI, 1988.

[56] A. Haraux \& E. Zuazua, Decay estimates for some semilinear damped hyperbolic problems, Arch. Rational Mech. Anal. 100 (1988) 191-206.

[57] D. Henry, Geometric theory of semilinear parabolic equations, Lecture Notes in Mathematics, 840. Springer-Verlag, Berlin - New York, 1981. 
[58] M. A. Jorge Silva \& T. F. Ma, On a viscoelastic plate equation with history setting and perturbation of $p$-Laplacian type, IMA J. Appl. Math. (submetido)

[59] M. A. Jorge Silva \& T. F. Ma, Finite-dimensional global attractor to a plate equation with memory and perturbation of p-Laplacian type (a submeter)

[60] M. A. Jorge Silva, T. F. Ma \& J. E. Muñoz Rivera, Analyticity and rates of decay to a fully and partially damped Mindlin-Timoshenko plate model (a submeter)

[61] A.Kh. Khanmamedov, Global attractors for von Karman equations with nonlinear interior damping. J. Math Anal Appl, 318 (2006), 92-101.

[62] J. U. Kim, A boundary thin obstacle problem for a wave equation, Comm. Partial Differential Equations 14 (1989) 1011-1026.

[63] E. Kreyszig, Introductory functional analysis with applications, John Wiley \& Sons, New York-London-Sydney, 1978.

[64] J. E. Lagnese, Boundary Stabilization of Thin Plates, SIAM Studies in Applied Mathematics, 10. Society for Industrial and Applied Mathematics (SIAM), Philadelphia, PA, 1989.

[65] J. E. Lagnese \& J. L. Lions, Modelling, analysis and control of thin plates, Collection RMA, Masson, Paris, 1988.

[66] J.-L. Lions, Quelques Méthodes de Résolution des Problèmes aux Limites Non Linéaires, Dunod Gauthier-Villars, Paris 1969.

[67] J.-L. Lions \& E. Magenes, Non-Homogeneous Boundary Value Problems and Applications, Vol.I, Springer-Verlag, Berlin, 1972.

[68] Z. Liu \& B. Rao, Characterization of polynomial decay rate for the solution of linear evolution equation, Z. angew. Math. Phys. 56, 630-644 (2005).

[69] Z. Liu \& J. Yong, Qualitative properties of certain $C_{0}$ semigroups arising in elastic systems with various dampings, Adv. Differential Equations 3 (1998), no. 5,

[70] Z. Liu \& S. Zheng, Semigroups associated with dissipative systems, Chapman \& Haal/CRC Research Notes in Mathematics v. 398, Boca Raton, FL, 1999.

[71] T. F. Ma, Boundary stabilization for a nonlinear beam on elastic bearings, Math. Meth. Appl. Sciences 24 (2001) 583-594.

[72] T. F. Ma \& J. A. Soriano, On weak solutions for an evolution equation with exponential nonlinearities, Nonlinear Anal. 37 (1999) 1029-1038.

[73] S. A. Maia \& M. Milla Miranda, Existence and decay of solutions of an abstract second order nonlinear problem, J. Math. Anal. Appl. 358 (2009) 445-456. 
[74] J. Málek \& D. Pražák, Large time behavior via the method of l-trajectories, J. Differ. Equations 181 (2002), no. 2, 243-279.

[75] L. A. Medeiros, Tópicos em Equações Diferenciais Parciais-Parte I, Instituto de Matemática UFRJ, Rio de Janeiro, 2005.

[76] L. A. Medeiros \& M. Milla Miranda, Espaços de Sobolev (Iniciação aos Problemas Elípticos não Homogêneos), Instituto de Matemática - UFRJ, Rio de Janeiro, 2000.

[77] S. A. Messaoudi, On the decay of solutions for a class of quasilinear hyperbolic equations with non-linear damping and source terms, Math. Methods Appl. Sci. 28 (2005) 1819-1828.

[78] J. E. Muñoz Rivera, Asymptotic behaviour in linear viscoelasticity, Quart. Appl. Math. 52 (1994) 628-648.

[79] J. E. Muñoz Rivera, Estabilização de Semigrupos e Aplicações, Séries de Métodos Matemáticos, Rio de janeiro, 2008.

[80] J. E. Muñoz Rivera \& H. P. Oquendo, Asymptotic behavior of a Mindlin-Timoshenko plate with viscoelastic dissipation on the boundary, Funkcial. Ekvac. 46 no. 3, 363-382 (2003).

[81] J. E. Muñoz Rivera \& R. Racke, Global stability for damped Timoshenko systems, Disc. Cont. Dyn. Sys. 9, 1625-1639 (2003).

[82] V. Pata \& S. Zelik, Global and exponential attractors for 3-D wave equations with displacement dependent damping, Math. Meth. Appl. Sci. 29 (2006), no. 11, 1291-1306.

[83] V. Pata \& A. Zucchi, Attractors for a damped hyperbolic equation with linear memory, Adv. Math. Sci. Appl. 11 (2001), no. 2, 505-529.

[84] J. Y. Park, H. M. Kim \& S. H. Park, On weak solutions for hyperbolic differential inclusion with discontinuous nonlinearities, Nonlinear Anal. 55 (2003) 103-113.

[85] A. Pazy, Semigroups of Linear Operators and Applications to Partial Differential Equations, Applied Mathematical Sciences v. 44, Springer-Verlag, New York, 1983.

[86] J. Prüss, On the spectrum of $C_{0}$-semigroups, Trans. Amer. Math. Soc. 284 (1984), no. 2, 847857.

[87] M. Sango, On a nonlinear hyperbolic equation with anisotropy: global existence and decay of solution, Nonlinear Anal. 70 (2009) 2816-2823.

[88] M. L. Santos \& C. C. S. Tavares, On the Kirchhoff plates equations with thermal effects and memory boundary conditions, Appl. Math. Comput. 213 (2009), no. 1, 25-38.

[89] J. Simon, Compact sets in the space $L^{p}(0, T ; B)$, Ann. Mat. Pura Appl. (4) 146 (1987), 65-96. 
[90] R. Temam, Navier-Stokes Equations, Theory and Numerical Analysis, North-Holland, Amsterdam, 1979.

[91] R. Temam, Infinite-Dimensional Dynamical Systems in Mechanics and Physics, Applied Mathematical Sciences 68, Springer- Verlag, New York, 1988.

[92] M. Tsutsumi, Some nonlinear evolution equations of second order, Proc. Japan Acad. 47 (1971), suppl. II, 950-955.

[93] K. Yosida, Functional Analysis (6th edition), Springer-Verlag, New York, 1980.

[94] S. Zheng, Nonlinear evolution equations, Chapman \& Haal/CRC Monographs and Surveys in Pure and Applied Mathematics v. 133, Boca Raton, FL, 2004.

[95] Yang Zhijian, Global existence, asymptotic behavior and blowup of solutions for a class of nonlinear wave equations with dissipative term, J. Differential Equations 187 (2003) 520-540.

[96] Yang Zhijian, Longtime behavior for a nonlinear wave equation arising in elasto-plastic flow, Math. Meth. Appl. Sci. 32 (2009) 1082-1104.

[97] Yang Zhijian \& Jin Baoxia, Global attractor for a class of Kirchhoff models, J. Math. Phys. 50, 032701 (2009) 29 pp.

[98] Yang Zhijian, Global attractors and their Hausdorff dimensions for a class of Kirchhoff models, J. Math. Phys. 51, 032701 (2010) 17 pp.

[99] Yang Zhijian, Finite-dimensional attractors for the Kirchhoff models, J. Math. Phys. 51, 092703 (2010) 25 pp.

[100] E. Zuazua, Exponential decay for the semilinear wave equation with locally distributed damping, Comm. Partial Differential Equations 15 (1990), no. 2, 205-235. 

Atrator global, 29, 87, 97

Conjunto

$\omega$-limite, 30

absorvente, 29, 87, 91

invariante, 29

positivamente invariante, 29, 91

Convergência

forte, 15

fraca, 14

fraca estrela, 14

Decaimento exponencial, 3, 48, 91

Decaimento polinomial, 7, 27, 118, 125

Desigualdade de

Gronwall, 17

Cauchy com $\epsilon, 18$

estabilização, 92

Gagliardo-Nirenberg, 16

Hölder, 17

Hölder Generalizada, 17

Poincaré, 17

Young, 17

Young $\operatorname{com} \epsilon, 17$

Dimensão fractal, 31, 92, 99

Dual topológico, 14

Equação de evolução, 27

Espaço bidual, 14

com peso, 12

reflexivo, 15

separável, 15

Espaço de

distribuições, 11

distribuições vetoriais, 13

funções teste, 11

Sobolev, 11

Espectro de um operador linear, 25

Estabilidade exponencial, 7, 26, 114

Função contrativa, 30

Gerador infinitesimal, 24

História de deslocamento relativo, 4, 53

Majorante essencial, 10

Memória, 3, 4, 35, 53

Núcleo da memória, 3, 33

Operador

$p$-Laplaciano, 19

associado a uma forma bilinear, 22

com potência fracionária, 23, 100

de derivação, 10

dissipativo, 25

Otimalidade, 7, 118, 127 
Problema de Cauchy, 27, 107

Problema de valor inicial, 27

Pseudométrica pré-compacta, 30

Resolvente de um operador linear, 25

Semi-distância de Hausdorff, 29

Semigrupo

analítico, 24

assintoticamente compacto, 30

assintoticamente suave, 29

Semigrupo linear, 24

analítico, 25, 26, 112

de classe $C_{0}, 24,111$

de contrações, 24

exponencialmente estável, 24

gerado por um operador $A, 24$

uniformemente limitado, 24

Semigrupo não linear de

classe $C_{0}, 28$

evolução, 29

Seminorma compacta, 31

Sistema de mindlin-Timoshenko, 6, 7, 106

Sistema dinâmico, 29, 87

Sistema dinâmico dissipativo, 29, 87

Solução fraca, 36, 56

Suporte de uma função, 10

Teorema de

Lax-Milgram, 18

Aubin-Lions, 18

Carathéodory, 37

compacidade fraca, 18

compacidade fraca estrela, 18

densidade, 15

Gagliardo-Nirenberg, 16

Green, 17

Hille-Yosida, 25

imersões de Sobolev, 15

interpolação, 16

Lumer-Phillips, 25
Rellich-Kondrachov, 16

Representação de Riesz-Fréchet, 18

Topologia

fraca, 14

fraca estrela, 14 
